\title{
INVESTIGATING REPTIN FUNCTION IN ACUTE MYELOID LEUKAEMIA
}

\author{
Elena Armenteros Monterroso
}

Developmental Biology and Cancer Section

Institute of Child Health

University College London

A thesis submitted for the Degree of Doctor of Philosophy 


\section{DECLARATION}

I, Elena Armenteros Monterroso, confirm that the work presented in this thesis is my own. Where information has been derived from other sources, I confirm that this has been indicated in the thesis.

Signature 


\section{ACKNOWLEDGEMENTS}

Firstly, I would like to express my sincere gratitude to my principal supervisor, Dr. Owen Williams, for his excellent advice, support and motivation during the past 4 years. I am extremely grateful for his guidance, but also for the freedom he has given me to pursue my own research. I could not have imagined having a better supervisor.

I would also like to extend my gratitude to my second supervisor, Dr. Jasper de Boer. His help and advice have been invaluable. But also the fun environment he has provided in the lab, which made it easier to carry on during stressful times. I am also thankful to all the inspirational people working at the Cancer Section, particularly all the members of my lab, for their help and friendship during the past years. My sincere thanks also goes to all the members of the UCL Genomics team for their efficient work and their help with my sequencing experiments.

I am also truly thankful to all my friends, both in the UK and in Spain, for providing the enthusiasm and support that I needed during my studies. I would like to specially thank Miriam, Clare and Heike for their friendship and fun times together. I am also deeply grateful to Ruben, for his love and relentless support during my $\mathrm{PhD}$.

My special gratitude goes to all my family members, who never stopped believing in me, with a special mention to my parents, for their unconditional love and motivation. I would like to dedicate this thesis to them - Muchas gracias! 


\section{ABSTRACT}

Acute myeloid leukaemia (AML) is a disease characterized by the clonal expansion of immature white blood cells, which show increased proliferation, self-renewal and a block of differentiation. Despite recent advances in therapy, AML still causes over half of all leukaemia related paediatric deaths, as cytogenetically defined subgroups with poor prognosis are still prevalent. Chromosomal translocations, which encode abnormal fusion proteins, are common in patients with AML, and the MLL (Mixed Lineage Leukaemia) locus is the most frequently rearranged in paediatric AML. Previous studies in our laboratory used global gene expression analysis in conditionally immortalized $M L L$-rearranged mouse myeloid cells to demonstrate that Reptin was positively regulated by $M L L$-fusion genes. Reptin (also known as RUVBL2 or Tip48), functions as part of multi-protein complexes involved in chromatin remodelling, DNA repair, regulation of transcription and ribonucleoprotein assembly. Further work in our laboratory found Reptin to be essential for sustaining the hyperproliferative state and clonogenic potential, as well as suppressing apoptosis, of human AML cells, both $M L L$-rearranged and non- $M L L$ rearranged.

The aim of this study was to investigate the transcriptional pathways regulated by Reptin in human AML and to establish the efficacy of targeting Reptin in vivo. By using an inducible shRNA model to deplete Reptin expression we demonstrate that Reptin is essential for leukaemic progression in vivo, as Reptin knockdown in established human leukaemias resulted in increased survival and the 
cure of most xenotransplanted mice. Moreover, our analyses of global gene expression data in cells depleted for Reptin expression at different time points indicated that Reptin depletion is negatively correlated with the Leukaemic Stem Cell self-renewal signature. Furthermore, our gene expression results also indicated that Reptin modulates the expression signature of the transcription factors c-MYC and cMYB, master regulators of survival and self-renewal pathways in AML. Additionally, immunoprecipitation assays identified a novel interaction between endogenous Reptin and c-MYB, and ChIP assays showed decreased binding of cMYB and the epigenetic mark H3K27ac at the promoter region of the c-MYB target gene $M P O$ after Reptin loss. Collectively, our data identify a new pathway modulated by Reptin and confirm that Reptin is a good therapeutic target for the treatment of AML. 


\section{TABLE OF CONTENTS}

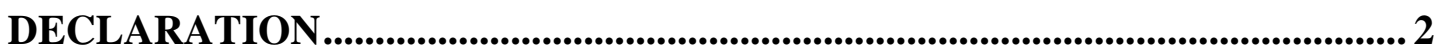

ACKNOWLEDGEMENTS.................................................................................. 3

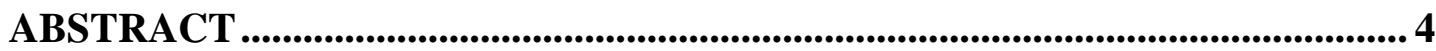

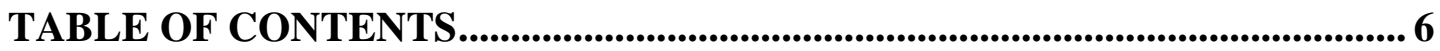

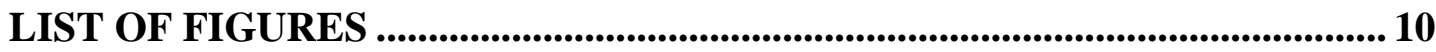

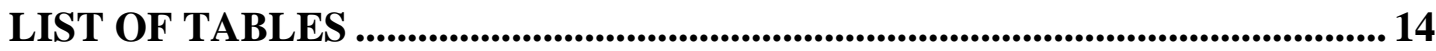

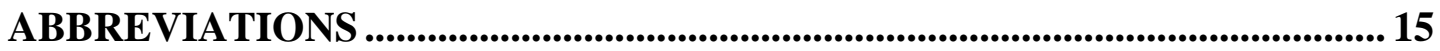

1 CHAPTER I - Introduction ......................................................................... 21

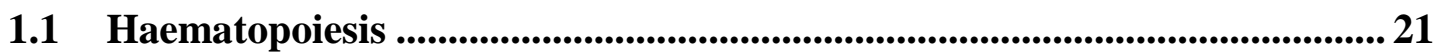

1.2 Childhood Acute Myeloid Leukaemia (AML) .........................................26

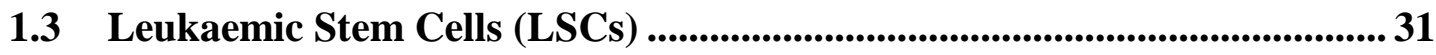

1.4 Molecular genetics in childhood AML .............................................................34

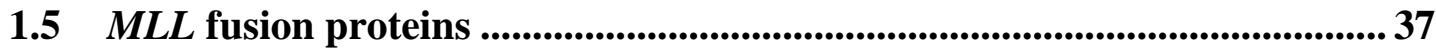

1.6 c-MYB in normal and aberrant haematopoiesis ......................................43

$1.7 \quad \mathrm{c}-\mathrm{MYC}$ in normal and aberrant haematopoiesis ......................................5 50

1.8 The ATPases Reptin (RUVBL2) and Pontin (RUVBL1) ......................... 58

1.8.1 Reptin and Pontin structure .......................................................... 60

1.8.2 Regulation of expression of Reptin and Pontin ............................... 65 
1.8.3 Reptin and Pontin function: chaperone-like activity in the assembly of several complexes

1.8.4 Reptin and Pontin function: regulation of gene transcription 70

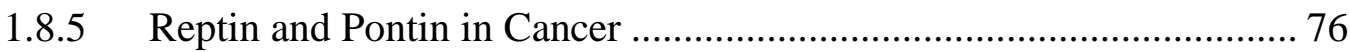

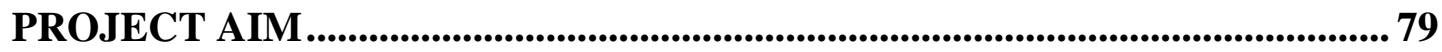

2 CHAPTER II - Materials and methods....................................................... 80

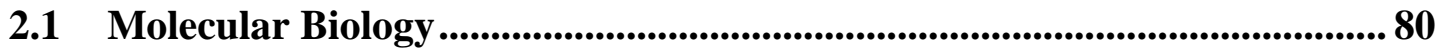

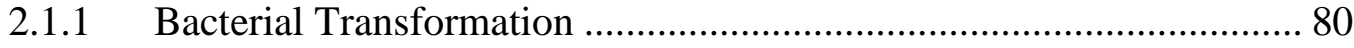

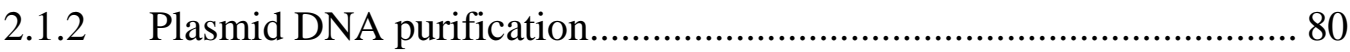

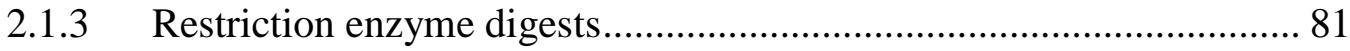

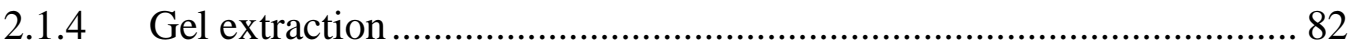

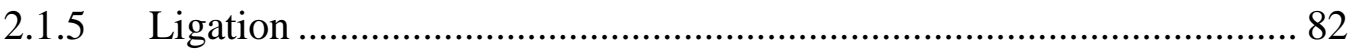

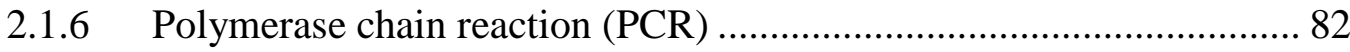

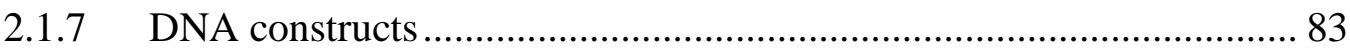

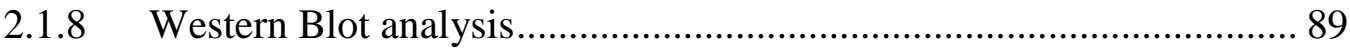

2.1.9 RNA isolation, cDNA preparation and qRT-PCR ........................... 90

2.1.10 Co-immunoprecipitation (Co-IP) assays ....................................... 91

2.2 Cell Biology ..................................................................................................... 93

2.2.1 Cell lines and tissue culture methods .............................................. 93

2.2.2 Lentiviral packaging cell line transfection ..................................... 94

2.2.3 Lentiviral transduction of target cells ............................................ 94 


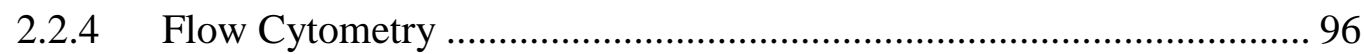

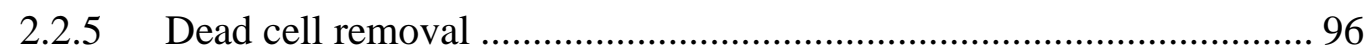

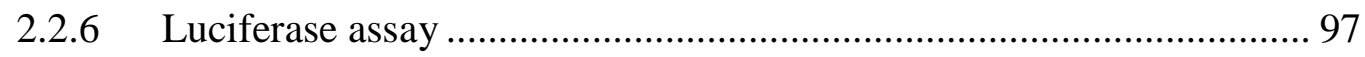

2.3 RNA-sequencing ................................................................................................. 97

2.3.1 RNA-seq Library preparation and Sequencing ..................................... 97

2.3.2 Gene set enrichment analysis (GSEA) …………………………...... 98

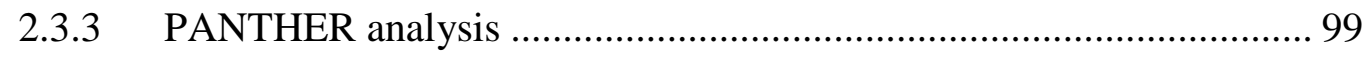

2.4 Chromatin immunoprecipitation (ChIP) assays.............................................99

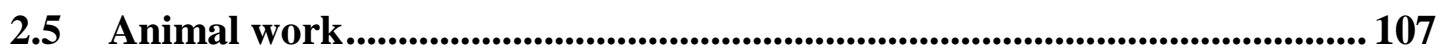

3 CHAPTER III - Investigating the consequences in vivo of conditional

Reptin loss on human AML ............................................................................................ 108

3.1 Introduction ............................................................................................................. 108

3.2 Results ................................................................................................................. 110

3.2.1 Validating Reptin knockdown in inducible THP-1 clones................. 110

3.2.2 Characterising luciferase transduced inducible THP-1 clones........... 112

3.2.3 Reptin depletion eliminates established leukaemia in vivo ................. 114

3.3 Discussion .................................................................................................................... 118

4 CHAPTER IV - Studying the molecular function of Reptin in human acute myeloid leukaemia.............................................................................................................. 123

4.1 Introduction .................................................................................................. 123

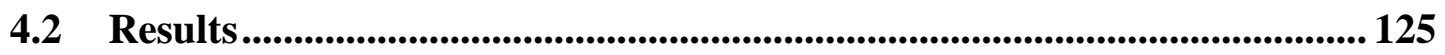


4.2.1 Examining the effect of Reptin depletion and depletion of Reptininteracting partners on c-MYC.

4.2.2 Reptin does not interact with c-MYC in AML cells

4.2.3 Characterising the response of bulk THP-1 cells to Reptin loss 145

4.2.4 Global gene expression analysis following Reptin silencing 154

4.2.5 Reptin modulates the LSC, c-MYC and c-MYB signatures 162

4.3 Discussion 172

4.3.1 Reptin regulates c-MYC protein and transcript levels 172

4.3.2 Negative enrichment of the LSC, c-MYC and c-MYB signatures after Reptin depletion 177

5 CHAPTER V - Investigating the role of Reptin on c-MYB function 182

5.1 Introduction 182

5.2 Results. 184

5.2.1 Validating the c-MYB negative enrichment after Reptin depletion .. 184

5.2.2 Reptin interacts with c-MYB in AML cells 201

5.2.3 Reptin decreases the binding of c-MYB to the chromatin 205

5.3 Discussion 209

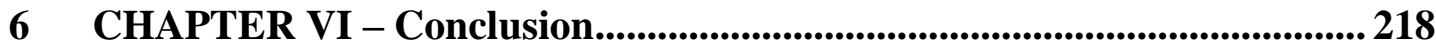

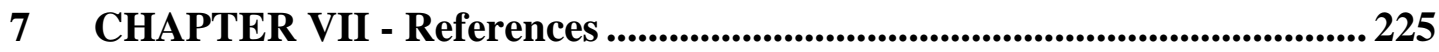

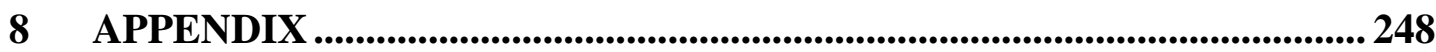




\section{LIST OF FIGURES}

Figure 1. Diagrams of the different models of haematopoiesis proposed................. 25

Figure 2. Schematic diagram of the MLL fusion oncoproteins. ............................. 43

Figure 3. Schematic showing the c-MYB protein domains.................................... 49

Figure 4. Schematic showing the functional domains of c-MYC ............................57

Figure 5. Reptin and Pontin regulate several cellular pathways.............................. 59

Figure 6. Structure and domains of Reptin and Pontin. .............................................. 63

Figure 7. Model proposed to explain the interplay between Reptin, Pontin and chromatin. 64

Figure 8. Lentiviral expression constructs used in this study. 84

Figure 9. Lentiviral expression constructs used in this study to constitutively knockdown gene expression.

Figure 10. Diagram of the pTRIPZ (PGK) inducible system used in this study. ...... 88

Figure 11. Standard curves of the primers used for ChIP assays........................... 105

Figure 12. Melt curves from qPCR of GD21 and MPO...................................... 106

Figure 13. Validation of Reptin depletion in THP-1 inducible clones. .................. 111

Figure 14. Characterisation of luciferase transduced inducible clones................... 113

Figure 15. Bioluminescence levels in all groups of mice at the start of the experiment.

Figure 16. Reptin depletion delays or abolishes leukaemia progression in vivo...... 116

Figure 17. Inducible Reptin depletion leads to decreased c-MYC protein in a THP-1 clone. 130

Figure 18. Reptin knockdown leads to c-MYC and Miz-1 loss. 131 
Figure 19. Overexpression of a Dominant Negative (D/N) mutant of Reptin leads to apoptosis and c-MYC protein loss

Figure 20. MTBP knockdown induces c-MYC protein degradation.

Figure 21. Knockdown of EPC1 and EPC2 induces c-MYC loss in THP-1 cells... 137

Figure 22. c-MYC transcript levels decrease after Reptin depletion.

Figure 23. Western blot analysis showing 3xHA-tagged Reptin overexpression.... 142

Figure 24. 3xHA-Reptin interacts with Pontin in THP-1 cells.

Figure 25. 3xHA-tagged Reptin does not interact with c-MYC

Figure 26. Inducible knockdown of Reptin in a bulk population of THP-1 cells .... 148

Figure 27. Time-course western blot analysis of Reptin protein. 150

Figure 28. Characterisation of the experiments used for RNA-sequencing at time point Day 2.

Figure 29. Characterisation of the experiments used for RNA-sequencing at time point Day 4.

Figure 30. RNA express workflow.

Figure 31. Analysis of differential gene expression after Reptin depletion. 158

Figure 32. Reptin depletion leads to increased expression of $C D 82$ 159

Figure 33. hTERT expression decreases after Reptin knockdown. 160

Figure 34. Gene Ontology and pathways analysis using PANTHER software. 161

Figure 35. GSEA plots show a negative correlation of the LSC signature after Reptin depletion 165

Figure 36. GSEA plots show a negative correlation of the c-MYC signature after Reptin depletion. 166 
Figure 37. Sequencing results show a decrease in $c-M Y C$ expression levels after 4 days of Reptin depletion.

Figure 38. GSEA plots show a negative correlation of the c-MYB signature after Reptin loss. 168

Figure 39. c-MYB transcript does not change after 4 days of Reptin depletion...... 169

Figure 40. Validation of c-MYB target genes after $c-M Y B$ loss 188

Figure 41. Validation of c-MYB target genes after inducible Reptin depletion. 189

Figure 42. Reptin protein levels after 7 days of constitutive Reptin depletion. 190

Figure 43. Reptin and CD82 transcript levels after 7 days of constitutive Reptin depletion. 191

Figure 44. Measurement of virus titre prior to RNA-sequencing 192

Figure 45. Upregulated and downregulated genes in common between two different RNA-seq experiments.

Figure 46. hTERT and CD82 $\log 2$ fold change after 7 days of constitutive Reptin depletion. 194

Figure 47. c-MYB transcript levels do not change after 7 days of constitutive Reptin depletion 195

Figure 48. GSEA plots of the $c-M Y B$ signature after 7 days of constitutive Reptin depletion. 196

Figure 49. Validation of selected c-MYB target genes by qRT-PCR after constitutive Reptin depletion. 197

Figure 50. Validation of selected c-MYB target genes by qRT-PCR after constitutive Reptin depletion with 2 additional shRNA sequences. 198

Figure 51. mRNA expression of c-MYB target genes after Pontin knockdown..... 200 
Figure 52. 3xHA-Reptin interacts with endogenous c-MYB in AML cell lines. .... 202

Figure 53. Endogenous Reptin protein interacts with endogenous c-MYB. 203

Figure 54. Endogenous Reptin immunoprecipitation in control and c-MYB depleted THP-1 cells. 204

Figure 55. c-MYB and Reptin protein levels after Reptin depletion in the samples used for ChIP assays. 207

Figure 56. Reptin depletion results in decreased c-MYB and H3K27ac binding at the promoter region the $M P O$ gene....... 208

Figure 57. Proposed model of Reptin function in AML cells 217 


\section{LIST OF TABLES}

Table 1. The French-American-British (FAB) classification of human AML.......... 29

Table 2. Table showing the WHO classification of AML subtypes. ......................... 31

Table 3. Cytogenetic abnormalities in adult vs childhood AML................................ 35

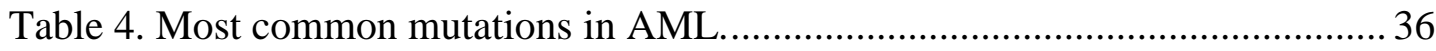

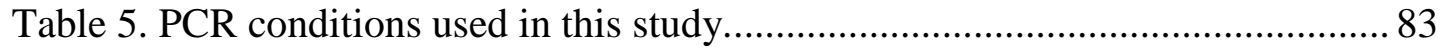

Table 6. List of pGIPZ shRNA sequences used in this study................................. 86

Table 7. List of MISSION® pLKO.1 shRNA sequences used in this study. ............. 86

Table 8. List of primary antibodies used for western blot analysis in this study....... 90

Table 9. List of antibodies used in this study to perform immunoprecipitation assays.

Table 10. List of antibodies used for ChIP assays in this study............................. 101

Table 11. List of primer sequences used for ChIP assays..................................... 102

Table 12. List of primer sequences tested for c-MYB ChIP assays........................ 104

Table 13. Top 30 enriched genes for the $c-M Y B$ activated GSEA signature. ........... 170

Table 14. List of the 100 most upregulated and downregulated genes after 4 days of inducible Reptin depletion 248

Table 15. List of the 100 most upregulated and downregulated genes after 7 days of constitutive Reptin depletion 253 


\section{ABBREVIATIONS}

AAA+

AF10

AF4

AF6

AF9

AGM

AKAP7

AML

$\mathrm{AMPr}$

ATM

ATR

Bp

bHLH-Zip

BSA

cDNA

ChIP

ChIP-seq

CLP

CMAP

CMP
ATPase belonging to the ATPase associated with various cellular activities

ALL1 fused gene from chromosome 10

ALL1 fused gene from chromosome 4

ALL1 fused gene from chromosome 6

ALL1 fused gene from chromosome 9

Aorta-Gonad-Mesonephros

A-Kinase Anchoring Protein 7

Acute Myeloid Leukaemia

Ampicillin resistance gene

Ataxia-Telangiectasia Mutated

ATM- and Rad3-Related

Base pair

Basic Helix-Loop-Helix Leucine Zipper

Bovine Serum Albumin

Complementary deoxyribonucleic acid

Chromatin immunoprecipitation

ChIP coupled to high-throughput sequencing analysis

Common Lymphoid Progenitor

Broad Institute Connectivity Map database

Common Myeloid Progenitor 


\begin{tabular}{|c|c|}
\hline c-MYB & Avian myeloblastosis viral oncogene homologue \\
\hline c-MYC & Avian myelocytomatosis viral oncogene homologue \\
\hline D/N Reptin & Dominant Negative mutant of Reptin \\
\hline DAPI & 4' 6-Diamidino-2-phenylindole \\
\hline $\mathrm{DBD}$ & DNA Biding Domain \\
\hline DMEM & Dulbecco's Modified Eagle's medium \\
\hline DNA & Deoxyribonucleic acid \\
\hline dNTPs & Deoxynucleotide triphosphate \\
\hline DSG & Disuccinimidyl glutarate \\
\hline DOX & Doxycycline \\
\hline DSS & Disuccinimidyl suberate \\
\hline DTT & Dithiothreitol \\
\hline DUSP4 & Dual Specificity Phosphatase 4 \\
\hline EDTA & Ethylenediaminetetraacetic acid \\
\hline EGTA & Ethylene Glycol Tetraacetic Acid \\
\hline ELL & Eleven-nineteen Lysine-rich Leukaemia \\
\hline ENL & Eleven Nineteen Leukaemia \\
\hline ES cells & Embryonic Stem cells \\
\hline EV & Empty Vector \\
\hline FCS & Foetal Calf Serum \\
\hline $\mathrm{Fc} \gamma \mathrm{RII} / \mathrm{III}$ & Fc $\gamma$ receptor-II/III \\
\hline FDR & False Discovery Rate \\
\hline GD21 & Gene Desert region on chromosome 21 \\
\hline GMP & Granulocyte-Monocyte restricted Progenitors \\
\hline
\end{tabular}




\begin{tabular}{|c|c|}
\hline GSEA & Gene Set Enrichment Analysis \\
\hline $\mathrm{H} 3 \mathrm{~K} 4$ & Histone H3 lysine 4 \\
\hline $\mathrm{H} 3 \mathrm{~K} 27 \mathrm{ac}$ & Acetylated Histone H3 lysine 27 \\
\hline HAT & Histone acetyltransferase \\
\hline $\mathrm{HCC}$ & Hepatocellular Carcinoma \\
\hline HLH & Helix-Loop-Helix \\
\hline HOX & Homeobox \\
\hline HPC & Haematopoietic Progenitor Cell \\
\hline HSCs & Haematopoietic Stem Cells \\
\hline hTERT & Human Telomerase Reverse Transcriptase \\
\hline IL-7R $\alpha$ & Interleukin-7 Receptor $\alpha$ chain \\
\hline IP & Immunoprecipitation \\
\hline IRES & Internal Ribosome Entry Site \\
\hline LB & Luria Broth \\
\hline Lin & Lineage-associated surface marker \\
\hline LMPP & Lymphoid-Primed Multipotent Progenitor \\
\hline LSC & Leukaemic Stem Cells \\
\hline LT-HSC & Long-Term haematopoietic stem cells \\
\hline LTR & Long Terminal Repeat \\
\hline LZ & Leucine Zipper \\
\hline $\mathrm{NaCl}$ & Sodium Chloride \\
\hline MAX & MYC-Associated Factor X \\
\hline MEIS1 & Myeloid Ecotropic viral Integration Site 1 \\
\hline MEP & Megakaryocyte-erythroid restricted prog \\
\hline
\end{tabular}




\begin{tabular}{|c|c|}
\hline MPP & Multipotent Progenitors \\
\hline MLL & Mixed Lineage Leukaemia \\
\hline MPO & Myeloperoxidase \\
\hline NRD & Negative Regulatory Domain \\
\hline NES & Normalised Enrichment Score \\
\hline NK & Natural Killer \\
\hline NOD/SCID & Non-Obese Diabetic/Severe Combined Immunodeficiency \\
\hline NTC & Non-Targeting Control \\
\hline PAFc & polymerase-associated factor complex \\
\hline PANTHER & Protein ANalysis THrough Evolutionary Relationships \\
\hline PBS & Phosphate-Buffered Saline \\
\hline PCR & Polymerase Chain Reaction \\
\hline PGK & Phosphoglycerate Kinase Promoter \\
\hline PHD & Plant Homeodomain \\
\hline PLD6 & Phospholipase D Family Member 6 \\
\hline $\mathrm{P}-\mathrm{TEFb}$ & Positive Transcription Elongation Factor b \\
\hline PVDF & Polyvinylidenefluoride \\
\hline qPCR & quantitative PCR \\
\hline qRT-PCR & quantitative Real-Time PCR \\
\hline $\mathrm{RCC}$ & Renal Cell Carcinoma \\
\hline $\mathrm{RD} 1$ & Repression domain 1 \\
\hline RD2 & Repression domain 2 \\
\hline RIPA & Radio Immunoprecipitation Assay \\
\hline RNA & Ribonucleic acid \\
\hline
\end{tabular}




\begin{tabular}{|c|c|}
\hline RNA-seq. & RNA coupled to high-throughput sequencing analysis \\
\hline RNP & Ribonucleoprotein \\
\hline Rpm & Revolutions Per Minute \\
\hline RPMI & Roswell Park Memorial Institute \\
\hline RRE & Rev-Response Element \\
\hline RT & Room Temperature \\
\hline rtTA3 & Reverse Tet-transactivator 3 \\
\hline SAMSN1 & SAM Domain SH3 Domain And Nuclear Localization \\
\hline & Signals 1 \\
\hline SEC & Super elongation complex \\
\hline SET & $\mathrm{Su}$ (var)3-9, Enhancer-of-zeste and Trithorax \\
\hline SD & Standard deviation \\
\hline SDS & Sodium Dodecyl Sulphate \\
\hline SDS-PAGE & SDS-polyacrylamide gel electrophoresis \\
\hline SEC & Super Elongation Complex \\
\hline SFFV & Spleen focus-forming virus \\
\hline shRNA & Short-hairpin ribonucleic acid \\
\hline $\operatorname{shSCR}$ & shScrambled \\
\hline shRep & shReptin \\
\hline SNL-1 & Nuclear localization signal 1 \\
\hline SNL-2 & Nuclear localization signal 2 \\
\hline ST-HSC & Short-Term Haematopoietic Stem Cell \\
\hline TAD & Transactivation Domain \\
\hline TRE & Tetracycline-Responsive promoter Element \\
\hline
\end{tabular}


TRIS

tRFP

WPRE

WNT

$\Psi$
Tris(hydroxymethyl)aminomethane

Turbo Red Fluorescent Protein

Woodchuck hepatitis posttranscriptional regulatory element

Wingless-type

Viral packaging signal 


\section{CHAPTER I - Introduction}

\subsection{Haematopoiesis}

Haematopoiesis refers to the process of blood cell formation occurring during embryonic development and adulthood to produce and replenish the blood system (Jagannathan-Bogdan and Zon, 2013). Development of the blood cells in mammals occurs in two different waves. The first one is called primitive haematopoiesis and its main function is to oxygenate the rapidly growing embryo. It begins in the yolk sac of the embryo and it then moves to an area surrounding the dorsal aorta - termed the aorta-gonad mesonephros (AGM) - and to the placenta. The largest expansion of embryo haematopoietic cells takes place in the foetal liver. The subsequent second wave is definitive haematopoiesis, and it occurs when cells colonise the spleen and thymus, and finally the bone marrow of the adult (Orkin and Zon, 2008). Haematopoiesis is generally conserved in vertebrates. Therefore, in order to better understand the process of blood formation in humans, it has been well studied using mouse models, although some differences have been described between mouse and human haematopoiesis (Iwasaki and Akashi, 2007a). The use of functional repopulation assays of human xenotransplantations has also helped to better understand blood formation in humans (Doulatov et al., 2012).

Haematopoiesis is a hierarchical process, whereby the haematopoietic stem cells (HSCs) present in the bone marrow, a rare population of cells which can selfrenew, give rise to progenitor cells and then to committed precursors of two distinct 
lineages: the myeloid and the lymphoid lineages (Orkin, 2000). The lymphoid lineage comprises $\mathrm{T}, \mathrm{B}$, and natural killer (NK) cells, while the myeloid lineage consists of granulocytes (neutrophils, eosinophils and basophils), monocytes/macrophages, erythrocytes, megakaryocytes and mast cells. Dendritic cells can develop from either the lymphoid or myeloid lineage (Iwasaki and Akashi, 2007b).

The myeloid lineage was proposed to be generated from Common Myeloid Progenitor cells (CMPs) deriving from short-term hematopoietic stem cells (STHSC) with limited self-renewal potential. In turn, these ST-HSCs derive from longterm hematopoietic stem cells (LT-HSC) which can undergo extensive self-renewal. In mice, HSCs form part of what is known as the LSK population, as they do not express lineage-associated-surface markers (Lin), but they do express stem cell antigen-1 (Sca-1) and c-Kit (Iwasaki and Akashi, 2007a). LT-HSCs in the LSK fraction of mouse bone marrow do not express the surface marker CD34 (CD34) (Osawa et al., 1996), but they do express CD38 (CD38 $\left.{ }^{+}\right)$(Randall et al., 1996) and low levels of Thy1.1 markers (Thy.1.1 ${ }^{\text {lo }}$ ) (Morrison and Weissman, 1994). In humans, however, the majority of LT-HSCs reside in the $\mathrm{Lin}^{-} \mathrm{CD} 34^{+} \mathrm{CD} 38^{-}$Thy $1^{+}$ $\mathrm{CD} 4 \mathrm{RA}^{-}$compartment (Doulatov et al., 2012), although a rare more primitive population of HSCs that do not express CD34 ( Lin $^{-}$CD34- CD38 ${ }^{-}$CD93 ${ }^{\text {hi }}$ ) has also been described (Anjos-Afonso et al., 2013). While CMPs also express high levels of CD34, they express low levels of the IL-7R $\alpha$, in contrast to the Common Lymphoid Progenitors (CLP), which generate the lymphoid lineage. As CMP cells differentiate, this fraction can be further divided on the basis of the $\mathrm{Fc}_{\mathrm{\gamma}}$ Receptor II/III expression 
(an important marker for myelomonocytic cells) into three distinct myeloidprogenitor subsets: $\mathrm{Fc}_{\gamma} \mathrm{RII} / \mathrm{III}^{\mathrm{lo}} \mathrm{CD} 34^{+} \mathrm{CMPs}, \mathrm{Fc}_{\gamma} \mathrm{RII} / \mathrm{III}^{\mathrm{lo}} \mathrm{CD} 34^{-}$megakaryocyteerythrocyte progenitors (MEPs), and $\mathrm{Fc}_{\gamma} \mathrm{RII} / \mathrm{III}^{\mathrm{hi}} \mathrm{CD} 34^{+}$granulocyte-monocyte restricted progenitors (GMPs) (Akashi et al., 2000) (Iwasaki and Akashi, 2007b). While the CMPs can generate all types of myeloid colonies, the GMPs can only generate granulocytes/macrophages and the MEPs can only generate megakaryocyte and erythrocyte lineage cells (Iwasaki and Akashi, 2007b). In contrast, CLP cells give rise to $\mathrm{B}$ and $\mathrm{T}$ cell progenitors (Pro- $\mathrm{B}$ and Pro- $\mathrm{T}$ ) that differentiate to $\mathrm{T}$ lymphocytes, B lymphocytes and NK cells. This is the classical model of haematopoiesis. However, this hierarchy has been challenged in the past few years. Using murine models of haematopoiesis, Adolfsson and colleagues identified a population of pluripotent HSC that lost their megakaryocyte-erythrocyte potential but retained the potential to develop into lymphoid primed multipotent progenitors (LMPP) that, upon loss of granulocyte-macrophage potential, generated CLPs (Adolfsson et al., 2001) (Adolfsson et al., 2005). This model has been termed the alternative model of haematopoiesis. There is a third one named the composite model, which combines the previous two models described. However, Yamamoto et al. recently described a new model called the myeloid bypass model. In this model, a self-renewing myeloid-restricted population of progenitor cells (MyRPs), located in the phenotypically defined HSC compartment, is lineage committed to megakaryocytes (MkRPs), megakaryocyte-erythroid (MERPs) or common myeloid (CMRPs) cells. These data suggested that HSCs can differentiate into myeloid lineage-restricted progenitors without passing through a multipotent progenitor (MPP) state (Yamamoto et al., 2013). Figure 1 shows diagrams of all the different 
models. Collectively, all of these studies suggest that the relationship between differentiation and lineage commitment during haematopoiesis may be more complex than at first envisaged. 
A)

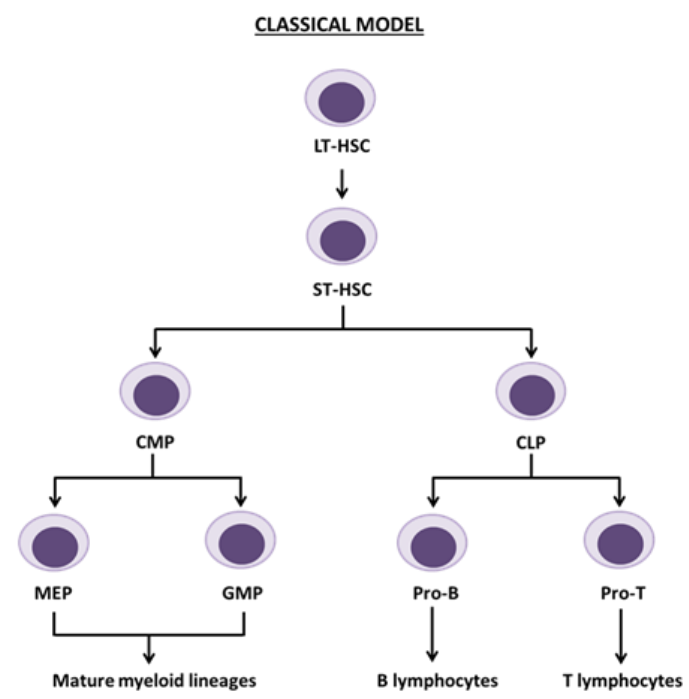

C)

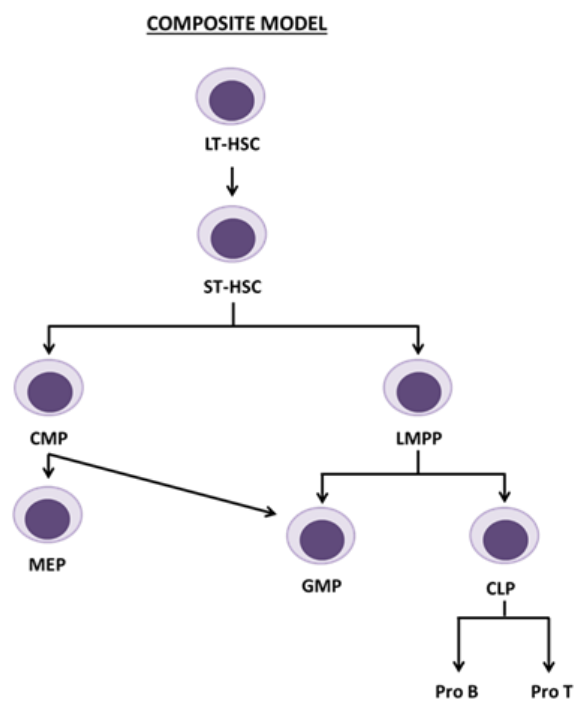

B)

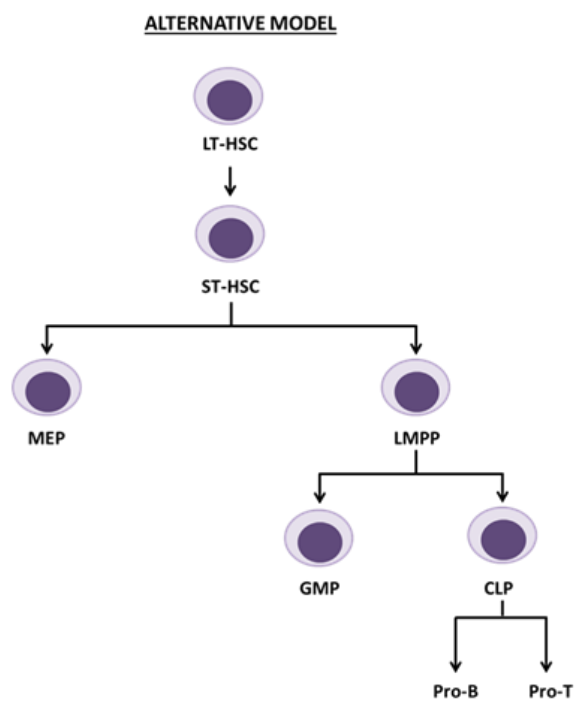

D)

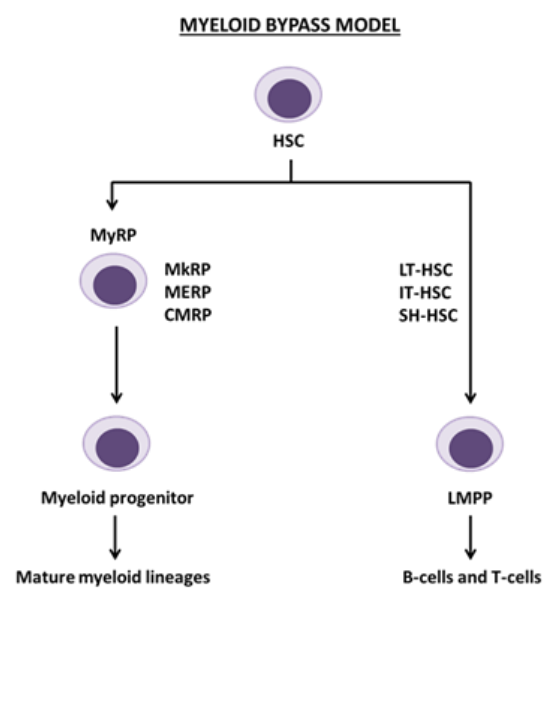

Figure 1. Diagrams of the different models of haematopoiesis proposed.

(A) In the classical model of haematopoiesis, long term HSCs (LT-HSCs) self-renew to maintain the HSC pool but also differentiate to short term HSCs (SH-HSCs) which have limited self-renewal capacity. They can differentiate into common myeloid progenitors (CMP) or common lymphoid progenitors (GMP). CMPs give rise to megakaryocyte-erythrocyte restricted progenitors (MEP) and granulocyte- 
macrophage restricted progenitors (GMP), which differentiate to mature myeloid lineage cell types. The CLP population gives rise to $\mathrm{B}$ and $\mathrm{T}$ cell progenitors (Pro-B and Pro-T), which mature to B and T lymphocytes, and NK cells. (B) The alternative model of haematopoiesis suggests a less lineage-restricted process and introduces a lymphoid-primed multipotent progenitor (LMPP) population. (C) In the composite model, CMPs and LMPPs coexist and are both capable of generating GMPs. (D) The myeloid bypass model proposes that the HSC compartment also contains a myeloidrestricted population of progenitor cells (MyRPs), which comprises the megakaryocyte (MkRPs), megakaryocyte-erythroid (MERPs) or common myeloid (CMRPs) progenitor cells. Adapted from (Iwasaki and Akashi, 2007b) and (Yamamoto et al., 2013).

\subsection{Childhood Acute Myeloid Leukaemia (AML)}

Acute myeloid leukaemia (AML) is a clonal neoplastic disorder that arises when the HSCs or progenitor cells lose the capacity to differentiate into mature myeloid cells. They produce abnormal white blood cells (blasts) instead, that rapidly build up in the bone marrow, hindering the production of normal blood cells. This results in hematopoietic insufficiency that leads to haemorrhage, fatigue, infections and fever (Lowenberg et al., 1999) (Zeisig et al., 2012).

AML affects nearly 3000 people in the UK every year and its incidence increases progressively with age (CRUK, 2014) (Lowenberg et al., 1999). In children, leukaemia is the most common cancer type and, although AML accounts 
for less than $20 \%$ of all the childhood leukaemias, it still causes over half of all leukaemia related paediatric deaths (Faulk et al., 2014). Due to treatment intensification, selective use of HSC transplantation, better supportive care and improved risk stratification, paediatric AML survival rates have increased from less than $20 \%$ in the 1970 s to around $55 \%$ nowadays. However, cure rates for some cytogenetic subgroups still remain very low (Rubnitz and Inaba, 2012).

AML is a heterogeneous disease that consists of different morphological and biological subtypes (Downing and Shannon, 2002). The primary diagnosis consists of the identification of leukaemic myeloblasts by examining their morphology, as the blasts have a round and irregular nuclei, distinct nucleoli and a small cytoplasm (Lowenberg et al., 1999). To correctly categorize the type of AML, different classification systems have been proposed. Decades ago the French-AmericanBritish (FAB) group developed a classification system which divides AML in 9 subtypes based on the morphologic appearance of the blasts and their cytochemical characteristics (Table 1) (Lowenberg et al., 1999). Later on, the World Health Organisation (WHO) developed another classification system that takes into account genetic, immunophenotypic, biological, and clinical features (Table 2). For cases that do not fulfil the WHO criteria for classification, the FAB classification is used under the "AML, not otherwise specified (NOS)" category (Walter et al., 2013).

AML therapy is divided into two stages. The first one is aimed at getting complete remission of the leukaemic blasts (less than 5\%), which is the only response that extends survival. This stage consists of intensive induction with 7 days 
of cytarabine plus 3 days of an anthracycline (" $7+3$ "). This is followed by post remission therapy, which consists of consolidation chemotherapy to kill any remaining leukaemic cells, and/or hematopoietic cell transplant (HCT) in poor-risk patients (Estey and Dohner, 2006) (Saygin and Carraway, 2017). Although the "7+3" is still the standard of care, in the recent years new driver mutations have been identified and novel therapies have been investigated, including novel chemotherapeutic agents, but also new more specific treatments such as epigenetic modifiers and immunomodulators (Saygin and Carraway, 2017). 


\begin{tabular}{ccc}
\hline FAB & NAME & ASSOCIATED \\
TYPE & Undifferentiated AML & TRANSLOCATION, GENE \\
\hline M0 & Myeloblastic leukaemia with & inv(3q26), EVII \\
M1 & minimal maturation & - \\
& Myeloblastic leukaemia & \\
M2 & with maturation & $\mathrm{t}(8 ; 21), A M L 1-E T O$ \\
M3 & Promyelocytic leukaemia & $\mathrm{t}(15 ; 17), P M L-R A R \alpha$ \\
M4 & Myelomonocytic leukaemia & $11 \mathrm{q} 23, M L L$ \\
M4eos & Myelomonocytic leukaemia & inv(16), CBF $-M Y H 11$ \\
M5 & with eosinophilia & $11 \mathrm{q} 23, M L L$ \\
M6 & Megakaryoblastic leukaemia & - \\
\hline
\end{tabular}

Table 1. The French-American-British (FAB) classification of human AML.

Table adapted from (Lowenberg et al., 1999). 


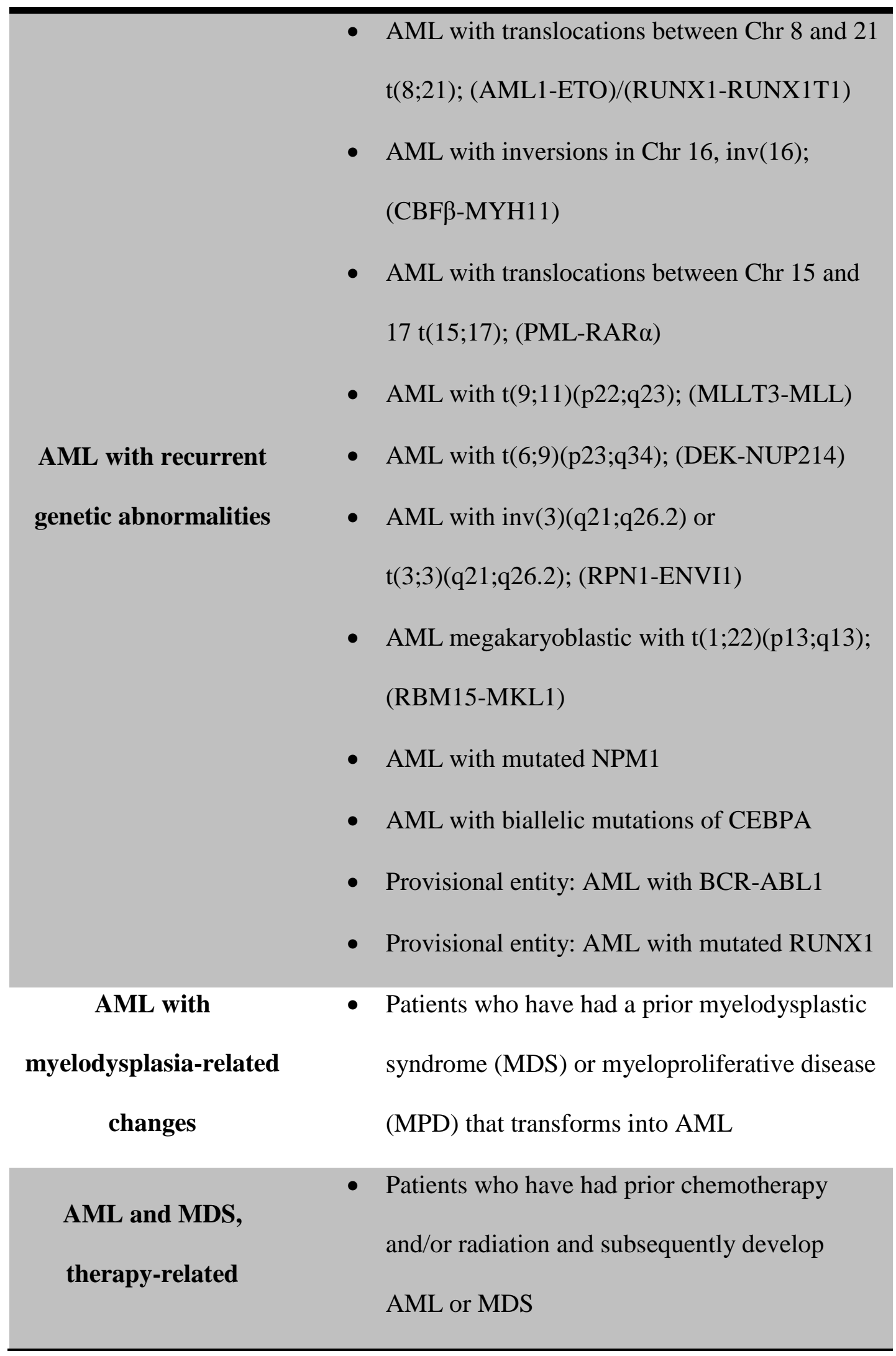


- Leukaemias may be characterised by specific chromosomal abnormalities

\begin{tabular}{|c|c|}
\hline $\begin{array}{l}\text { AML not otherwise } \\
\text { specified ("NOS") }\end{array}$ & $\begin{array}{l}\text { - Subtypes of AML that do not fall into the } \\
\text { above categories }\end{array}$ \\
\hline Myeloid Sarcoma & $\begin{array}{l}\text { Myeloid sarcoma remains as a unique clinical } \\
\text { presentation of any subtype of AML. }\end{array}$ \\
\hline $\begin{array}{l}\text { Myeloid proliferations } \\
\text { related to }\end{array}$ & $\begin{array}{l}\text { - Transient abnormal myelopoiesis AML } \\
\text { associated with Down syndrome }\end{array}$ \\
\hline
\end{tabular}

Down syndrome

Table 2. Table showing the WHO classification of AML subtypes.

Table adapted from (Vardiman et al., 2009), (Gruber, 2012) and (Arber et al., 2016).

\subsection{Leukaemic Stem Cells (LSCs)}

AML is a clonal disorder that has been proposed to be initiated from a rare Leukaemic Stem Cell (LSC) population, following a hierarchy similar to the one occurring in normal haematopoiesis. LSCs are cells with indefinite self-renewal potential that drive the formation of the leukaemia and sustain the disease by activating an abnormal differentiation programme. It is this aberrant differentiation programme that leads to the production of the leukaemic blasts (Bonnet, 2005) (Huntly and Gilliland, 2005). 
LSCs were initially termed SCID-leukaemia initiating cells (SL-IC) and were shown to express similar markers to HSCs, as only $\mathrm{CD}^{2} 4^{+} / \mathrm{CD}^{-} 8^{-}$cells (and not the more differentiated counterparts $\mathrm{CD} 34^{+} / \mathrm{CD} 38^{+}$) were found to be capable of transplanting leukaemia in NOD/SCID mice and giving rise to AML in secondary recipients (Bonnet and Dick, 1997) (Blair et al., 1998) (Lapidot et al., 1994). However, further work with more immune deficient mouse strains proved LSC activity in the more mature $\mathrm{CD} 34^{+} / \mathrm{CD} 38^{+}$fraction of some AML samples, although limiting dilution experiments showed that the frequency of LSCs in this population was lower than in the $\mathrm{CD}^{+} 4^{+} \mathrm{CD} 38^{-}$population (Taussig et al., 2008) (Horton and Huntly, 2012). In line with this, a more recent study sorted AML cells from patients according to their CD34/CD38 expression and found that the LSCs in all 4 sorted combinations showed leukaemia-initiating potential. However, in agreement with earlier studies, the majority of LSCs were found in the $\mathrm{CD} 34^{+} / \mathrm{CD} 38^{-}$fraction $(\mathrm{Ng}$ et al., 2016). Remarkably, Terwijn and colleagues found that the frequency of $\mathrm{CD} 4^{+} / \mathrm{CD} 38^{-}$neoplastic cells had an important prognostic impact, as higher percentages of these cells after treatment correlated with shorter patient survival (Terwijn et al., 2014). Collectively, all these studies suggest that LSCs might exist in all $\mathrm{CD} 34 / \mathrm{CD} 38$ populations, although the $\mathrm{CD} 34^{+} / \mathrm{CD} 38^{-}$fraction might be the most therapeutically relevant.

In addition to CD34 and CD38, LSCs have also been shown to express other more specific surface markers, including some myeloid antigens such as CD33 or CD123, and novel markers such as CLL-1, CD96 or TIM3, among others (Horton and Huntly, 2012). However, not all the LSC markers were found to be expressed to 
the same extent in different AML samples (Hanekamp et al., 2017). In order to further characterise the LSCs, several gene expression experiments have been performed using mouse LSCs models. Somerville et al. used a mouse model that recapitulates human $M L L$-driven AML and, surprisingly, they found that the LSCs in the model have a genetic signature more similar to embryonic stem cells than to adult HSCs. Moreover, LSCs in this particular model were also found to be metabolically active cycling cells, rather than quiescent (Somervaille et al., 2009). In a different study, LSCs were isolated from leukaemias initiated by $M L L-A F 9$ transduced committed GMPs and were found to have a similar gene expression signature than normal GMPs, but with the extra expression of a subset of genes important for selfrenewal normally highly expressed in HSCs (Krivtsov et al., 2006). Interestingly, this self-renewal signature was observed by Kvinlaug and colleagues in preleukaemic and leukaemic stem cells of acute myeloid leukaemias initiated by a diverse number of oncogenes, indicating that a common signature in LSCs is expressed independently of the driving mutation (Kvinlaug et al., 2011). The Hoxa9, Meis1, $c-M y b$ and $C b x 5$ genes were found to be important contributors of this selfrenewal signature (Kvinlaug et al., 2011) (Somervaille et al., 2009).

Several studies have found an association between LSC number and prognosis in AML patients, as a higher frequency of LSCs was found to correlate with a poorer clinical outcome (Majeti et al., 2009) (Eppert et al., 2011) (de Jonge et al., 2011). However, LSC may be more difficult to target with conventional chemotherapy, due to differences in their properties to bulk AML blasts, and they may therefore be the cause of many disease relapses (Costello et al., 2000) (Pollyea 
et al., 2014). Thus, the identification of the clinical significance of LSCs and the hierarchy in AML suggests that more specific therapies are needed that target the therapy-resistant LSC population rather than the bulk of the leukaemia (Horton and Huntly, 2012) (Hanekamp et al., 2017).

\subsection{Molecular genetics in childhood AML}

Molecular genetic alterations and cytogenetic abnormalities, leading to fusion genes, are very common in AML. Characterisation of all the genetic aberrations in AML has led to the conclusion that many of the genes affected also have a role in normal haematopoiesis and are involved in the aberrant proliferation, differentiation and apoptosis of blood cell precursors during AML (Mrozek et al., 2004). Interestingly, the frequency of these aberrations in adult and childhood AML is different. For example, while $45 \%$ of adult AML cases present a normal karyotype, only $20 \%$ of childhood AML cases are cytogenetically normal. In contrast, some cytogenetic abnormalities are much more common in paediatric AML. Translocations of the mixed lineage leukaemia (MLL) gene, for instance, are much more frequent in childhood and infant AML than in adult AML (Zeisig et al., 2012) (Pui et al., 1995). Table 3 shows a comparison of the different cytogenetic abnormalities affecting adult and childhood leukaemias. Although chromosomal translocations are very common in AML, they are believed to be important initiating drivers necessary to maintain the leukaemic phenotype but insufficient to induce a full leukaemic phenotype on their own. Thus, other genetic and epigenetic alterations are found alongside cytogenetic abnormalities in many cases (Gruber, 2012). However, the number of mutations or 
genetic lesions (e.g. deletions) found in paediatric AML is normally much lower than in other types of childhood leukaemias or than in other tumour types in adults (Radtke et al., 2009). Table 4 shows the most common mutated genes in AML.

\begin{tabular}{ccc}
\hline CYTOGENETIC & ADULT & CHILDHOOD \\
ABERRATION & AML & AML \\
\hline Normal karyotype & $41 \%$ & $20 \%$ \\
\hline t(15:17); (PML/ RAR $\alpha)$ & $13 \%$ & $10 \%$ \\
\hline t(8:21); (AML1-ETO) & $7 \%$ & $10 \%$ \\
inv(16); (CBFß-MYH11) & $5 \%$ & $5 \%$ \\
\hline 11q23; (MLL fusions) & $4 \%$ & $10 \%$ \\
Others & $12 \%$ & $28 \%$ \\
\hline Trisomy chromosome 21 & $3 \%$ & $5 \%$ \\
\hline Monosomy chromosome 7 & $5 \%$ & $4 \%$ \\
\hline Trisomy chromosome 8 & $10 \%$ & $8 \%$ \\
\hline
\end{tabular}

Table 3. Cytogenetic abnormalities in adult vs childhood AML.

Table showing a comparison of the most common cytogenetic abnormalities in adult and in children. Adapted from (Zeisig et al., 2012) and (Grimwade et al., 2010). 


\begin{tabular}{|c|c|c|}
\hline GENE & MUTATIONS IDENTIFIED & ADVANTAGE \\
\hline FLT3 & $\begin{array}{l}\text { Internal tandem duplication } \\
\text { Tyrosine kinase domain }\end{array}$ & Proliferative \\
\hline cKIT & Activating mutations & Proliferative \\
\hline RAS & Activating mutations & Proliferative \\
\hline PTPN11 & Activating mutations & Proliferative \\
\hline JAK2 & Activating kinase mutations & Proliferative \\
\hline JAK3 & Activating kinase mutations & Proliferative \\
\hline MPL & Activating cytokine receptor mutation & Proliferative \\
\hline AML1 & $\mathrm{t}(8 ; 21)$, point mutations & Differentiation arrest \\
\hline CEBPA & Truncated protein, DNA-binding site & Differentiation arrest \\
\hline WT1 & Insertions, deletions, point mutations & Differentiation arrest \\
\hline GATA1 & Truncated protein (GATA1s) & $\begin{array}{c}\text { Differentiation arrest } \\
\text { Proliferation advantage } \\
\text { in foetal progenitors }\end{array}$ \\
\hline NPM1 & $\begin{array}{l}\text { Translocations, deletions, point } \\
\text { mutations }\end{array}$ & Survival \\
\hline TP53 & Deletions, inactivating mutations & Survival \\
\hline
\end{tabular}

Table 4. Most common mutations in AML.

Table showing the genes that are frequently mutated in AML and the type of mutation normally found. The table also shows the advantage that the mutation confers to the leukaemic cells. Adapted from (Gruber, 2012). 


\section{5 $M L L$ fusion proteins}

Between $35 \%$ and $50 \%$ of infant AML cases are caused by chromosomal rearrangements involving the $M L L$ gene. Older children and adults also present these translocations, which account for the $10 \%$ of all AML cases (Muntean and Hess, 2012). These chromosomal translocations generate new oncogenic fusion proteins that initiate the leukaemic process, and the resulting leukaemias are generally very aggressive and with poor prognosis (Krivtsov and Armstrong, 2007) (Slany, 2009). Besides chromosomal translocations, partial tandem duplications (PTD) and amplification of the $M L L$ have also been described in acute leukaemias (Muntean and Hess, 2012) .

The MLL gene is located at chromosome band 11q23 and encodes a transcription factor with homology to the product of the Drosophila melanogaster trithorax gene (Zeleznik-Le et al., 1994). It belongs to the trithorax group of proteins (trxG), which activate gene transcription and antagonize the function of the Polycomb (PcG) group proteins (Muntean and Hess, 2012). The normal function of MLL in early haematopoietic progenitors is to modify the chromatin to activate gene transcription (Muntean and Hess, 2012). Chromatin is composed of DNA wrapped around an octamer of proteins called histones, which include $\mathrm{H} 2 \mathrm{~A}, \mathrm{H} 2 \mathrm{~B}, \mathrm{H} 3$ and $\mathrm{H} 4$ or some variants. The N-terminal domain of the histones can be modified by posttranslational modifications to regulate gene expression (Eissenberg and Shilatifard, 2010). MLL proteins methylate histone 3 lysine 4 (H3K4) residues to positively regulate and maintain the expression of several genes, including the homeobox 
$(H O X)$ genes, to stimulate the growth of progenitor cells (Muntean and Hess, 2012). The expression of these genes is later on downregulated when terminal differentiation is induced in normal haematopoiesis (Pineault et al., 2002). In $M L L$ rearranged leukaemias, the translocations also regulate the expression of the Hoxa genes, but the normal repression of their transcription does not happen (Horton et al., 2005). The constant expression of these genes, together with the upregulated expression of other genes, induces leukaemogenesis (Hess, 2004).

The $M L L$ gene encodes a very large protein which is cleaved by an aspartase protease (TASPASE1) into two fragments: a larger 320kDa $\mathrm{N}$-terminal fragment $\left(\mathrm{MLL}^{\mathrm{N}}\right)$ and a smaller 180kDa fragment, the C-terminal MLL $\left(\mathrm{MLL}^{\mathrm{C}}\right)(\mathrm{Hsieh}$ et al., 2003a) (Hsieh et al., 2003b). Both fragments are components of a large macromolecular complex which includes other partner proteins important for efficient gene transcription. In particular, in the $\mathrm{MLL}^{\mathrm{C}}$ fragment there is a transcriptional activation domain which interacts with at least 4 proteins (MOF, WDR5, RBBP5 and ASH2L) which modify the chromatin to prepare it for efficient transcription (Slany, 2009). Moreover, at the C-terminus of the $\mathrm{MLL}^{\mathrm{C}}$ fragment there is also a domain responsible for its function in methylating $\mathrm{H} 3 \mathrm{~K} 4$, named the SET domain (Milne et al., 2002). The $\mathrm{MLL}^{\mathrm{N}}$ fragment, in contrast, contains domains for its nuclear localisation (SNL-1 and SNL-2), transcriptional repression (RD1 and RD2), and for DNA binding (AT-hook motifs and a cysteine-rich CXXC domain). Next to the CXXC domain there are 4 plant homeodomain (PHD) fingers, important for protein-protein interactions, and a bromodomain, which mediates the binding to acetylated histones (Winters and Bernt, 2017). Importantly, the $\mathrm{MLL}^{\mathrm{N}}$ fragment 
interacts with two proteins (Menin and LEDGF), which tether the MLL complex to chromatin, and this interaction has been found to be maintained during leukaemogenesis (Caslini et al., 2007) (Yokoyama and Cleary, 2008). Another key interaction maintained during leukaemogenesis that directs MLL to the target loci is with the polymerase-associated factor complex (PAFc). PAFc is a transcriptional activation complex that mono ubiquitylates lysine 20 of histone $\mathrm{H} 2 \mathrm{~B}$, which is necessary for the histone methylation function of the MLL complex (Muntean and Hess, 2012).

In leukaemias, the $M L L$ fusion oncogenes resulting from chromosomal translocations always include a 5' fragment of the $M L L$ gene, encoding part of the $\mathrm{N}$ terminal moiety, fused to a variable number of exons from a fusion partner gene. Thus, the MLL fusion proteins retain the Menin binding domain, the AT-hooks, the nuclear localisation domains and the repression domains, including the one necessary to bind PAFc. However, they do not retain the PHD fingers, the transcriptional activation domain or the SET domain (Figure 2) (Winters and Bernt, 2017). Over 60 different $M L L$ fusion partner genes, capable of transforming the truncated MLL into a potent transcriptional activator, have been described (Zeisig et al., 2012). Nonetheless, there are six that account for more than two-thirds of all $M L L$ rearranged leukaemias. These are the $\mathrm{t}(4 ; 11)(M L L-A F 4), \mathrm{t}(9 ; 11)(M L L-A F 9)$, $\mathrm{t}(11 ; 19)(M L L-E N L), \mathrm{t}(10 ; 11)(M L L-A F 10), \mathrm{t}(11 ; 19)(M L L-E L L)$ and $\mathrm{t}(6 ; 11)(M L L-$ AF6), which encode nuclear proteins able to bind DNA and modify gene transcription (Slany, 2005) (Meyer et al., 2013) (Krivtsov and Armstrong, 2007). Of those, AF9 is the most common MLL fusion partner found in paediatric AML, 
followed by AF10 (Harrison et al., 2010) (Gruber, 2012). Although these MLL partners show little sequence similarity, AF4, AF9, ENL, AF10 and ELL were all found to participate in transcriptional elongation by forming part of a large macromolecular complex, named the super elongation complex (SEC). The SEC also includes other known transcription elongation factors, such as $\mathrm{P}-\mathrm{TEFb}$, which phosphorylates the C-terminal domain of the RNA Pol II (Smith et al., 2011). Interestingly, the 5'part of $M L L$ that is retained in the leukaemic chimeras does not contain the region encoding the methyltransferase activity of the protein, suggesting that the function of the MLL moiety in the fusion proteins is to recruit MLL-fusions to target loci, via interaction with proteins such as Menin and LEDGF (Lin et al., 2010). Other interacting proteins found to be important for $M L L$ fusion-induced leukaemogenesis are the bromodomain-containing proteins BRD3 and BRD4, which bind acetyl-lysine residues; the histone methyltransferase DOT1L, which methylates H3K79; and the Polycomb PRC1 complex component CBX8, important for gene repression (de Boer et al., 2013) (Winters and Bernt, 2017). Figure 2 shows a simplified schematic representation of the MLL fusion oncoproteins.

Several studies have shown that MLL fusion proteins induce both lymphoblastic and myeloid leukaemic transformation by maintaining upregulated expression of A-cluster Hox genes, particularly HoxA7, HoxA9, and the Hox cofactor Meis1 (Muntean and Hess, 2012). Gene expression profile experiments showed the upregulation of $H O X A$ genes expression in $M L L$-rearranged leukaemias (Armstrong et al., 2002) (Yeoh et al., 2002) (Ferrando et al., 2003). At the same time, it was shown that HoxA7 and HoxA9 are required to immortalise myeloid 
progenitors in vitro with the MLL fusion proteins. Moreover, HoxA9 was found to be required to induce MLL-leukaemogenesis in vivo, as Hoxa9 null murine bone marrow cells transduced with the $M L L-E N L$ fusion did not induce leukaemia in recipient mice (Ayton and Cleary, 2003). Using an inducible model of MLL-fusion leukaemia, Zeisig and colleagues also showed increased expression of HoxA genes in transformed cells. Furthermore, overexpression of HoxA9 with Meisl induced differentiation block and continuous proliferation in cells that had lost expression of the MLL-fusion protein (Zeisig et al., 2004). Similarly, a study from our group used an inducible model of MLL induced leukaemia in primary murine progenitor cells to show that the loss of the MLL-ENL fusion protein leads to decreased expression of Hox genes (Horton et al., 2005). Interestingly, LSCs in a mouse model of MLL-AF9 induced leukaemia were also found to express high levels of Hox genes (Somervaille and Cleary, 2006) and Meis1 was found to be an essential regulator of LSCs in a model of MLL induced leukaemia (Wong et al., 2007). In addition to this, suppression of HOXA9 in $M L L$-rearranged cell lines led to apoptosis and a reduced number of colonies in colony forming assays (Faber et al., 2009). However, in a different study depletion of HoxA9 and Meis 1 expression in murine $M L L$-rearranged AML cells did not affect proliferation of the cells in competitive proliferation assays. This result suggested that HoxA9 and Meis1 might be less important for MLLrearranged leukaemias than initially thought (Zuber et al., 2011). Interestingly, in that study the authors also found that MLL-AF9 contributes to leukaemia maintenance by enforcing a genetic signature similar to the transcription factor cMYB. Moreover, MLL-AF9 was found to directly regulate $c-M y b$ transcription by binding to its promoter. Other genes found to be directly regulated by the MLL-AF9 
fusion included Meis1, Irx5, FoxP1 and $c$-Myc among others (Zuber et al., 2011). Thus, besides deregulated Hoxa gene expression, other target genes regulated by the MLL fusion proteins appear to be important for AML progression. A different study found that $c-M y b, H m g b 3$ and $C b x 5$ genes were sufficient for immortalisation of myeloid progenitors in the absence of Hoxa gene upregulation (Somervaille et al., 2009). Further studies found that the transcription factor c-MYB regulates essential genes for survival of $M L L$-induced acute myeloid leukaemias. For example, in two independent studies, our group found that the c-MYB induced genes Frat1/2 and Reptin are also essential for MLL-fusion induced oncogenicity and survival (WalfVorderwulbecke et al., 2012) (Osaki et al., 2013). In this thesis, we have further investigated one of those targets, the ATPase Reptin, using an MLL-AF9 expressing AML paediatric cell line as a model. Our investigations have shown the important role of Reptin in regulating the function of two key proteins in AML, the transcription factors c-MYC and c-MYB. 


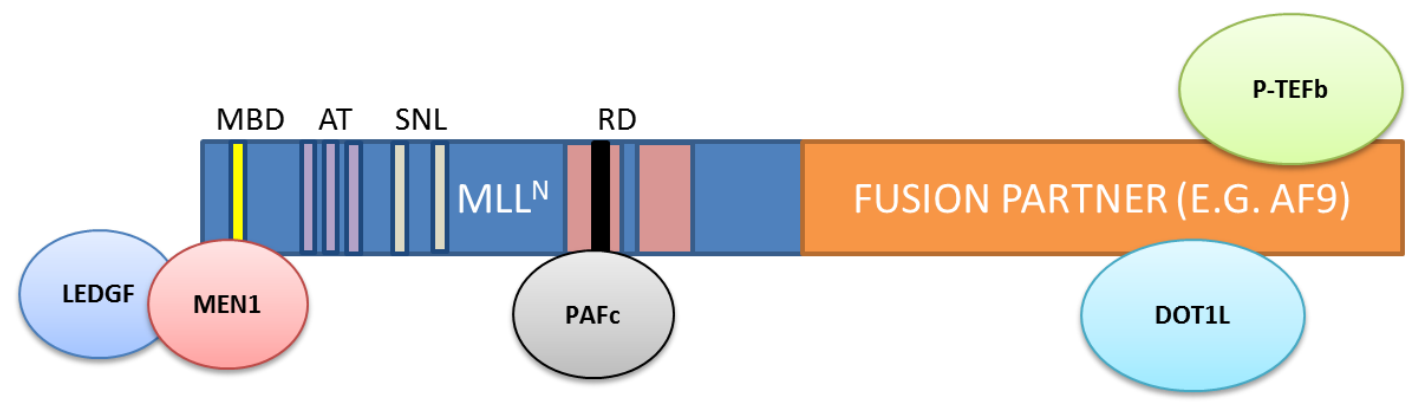

Figure 2. Schematic diagram of the MLL fusion oncoproteins.

The MLL fusion proteins are formed by the fusion of part of the N-terminal domain of the MLL and a fusion partner protein, such as AF9. Several other chromatin modifying proteins form a complex with the MLL fusion to aberrantly activate gene expression. MBD, Menin (MEN1) binding domain; AT, AT hooks; SNL, Speckled nuclear localisation domains; RD, Repression domains. Adapted from (Winters and Bernt, 2017).

\section{6 c-MYB in normal and aberrant haematopoiesis}

c-MYB, a downstream transcriptional target of the $M L L$-fusion oncoproteins, is a transcription factor with a central role in the regulation of normal haematopoiesis and in leukaemogenesis. It is the cellular homolog of $v-M Y B$, which was initially described as an oncogene causing leukaemia in chickens (Moscovici et al., 1975) (Pattabiraman and Gonda, 2013). It forms part of a family of transcription factors together with $A-M Y B(M Y B L 1)$ and $B-M Y B$ (MYBL2) (Uttarkar et al., 2017). As transcription factors, MYB proteins bind DNA sequences to regulate gene expression through a highly conserved N-terminal DNA biding domain (DBD). The specific 
motif recognised by these proteins, known as MYB binding site (MBS), has been described as t/cAACt/gG (Biedenkapp et al., 1988) (Ramsay and Gonda, 2008). The c-MYB protein also has a transactivation domain (TAD) in the centre of the protein, and a negative regulatory domain (NRD) in the C-terminal region (Figure 3) (Uttarkar et al., 2017).

Several proteins have been reported to interact with different c-MYB domains to modulate its activity. The histone acetyltransferases (HATs) CREBbinding protein $(\mathrm{CBP}) / \mathrm{p} 300$ bind the TAD of c-MYB and play a central role in regulating c-MYB function. They act as co-activators by modifying the chromatin, acetylating c-MYB and by bridging c-MYB with the transcription machinery (Greig et al., 2008). In contrast, the histone acetyltransferase TIP60 was found to interact with the TAD of c-MYB but negatively modulate its function (Zhao et al., 2012). Moreover, c-MYB has been found to co-operate or compete with other transcription factors. For instance, the transcription factors C/EBP $\alpha$ and PU.1, which are important for myeloid lineage commitment, were found to co-operate with c-MYB to activate the expression of the murine neutrophil elastase $(N E)$ gene in immature murine myeloid cells (Oelgeschlager et al., 1996). In a different example, c-MYB was found to compete with the transcription factor GATA-1 (which drives erythropoiesis) for the binding to CBP, thus inhibiting GATA-1 transcriptional activity (Takahashi et $a l ., 2000)$.

$c-M Y B$ is highly expressed in immature hematopoietic cells and it has been found to control hematopoietic cell proliferation, differentiation and survival (Zhou 
and Ness, 2011). Early work from the 1980s in murine and human cells already suggested a function of $c-M Y B$ in haematopoiesis, since higher expression of $c-M Y B$ was found in immature haematopoietic cells and in leukaemic cells. Besides, $c-M Y B$ expression was found to decrease upon differentiation into the different lineages (Westin et al., 1982) (Sheiness and Gardinier, 1984) (Gonda and Metcalf, 1984). Consistent with this, exogenous $c-M Y B$ overexpression was found to block differentiation of murine erythroid and myeloid cell lines (Clarke et al., 1988) (Yanagisawa et al., 1991). Another study exposed normal human progenitor cells to $c-M Y B$ antisense oligodeoxynucleotides to deplete its expression, and found a significant reduction in the size and number of colonies formed (Gewirtz and Calabretta, 1988). Later on, $c-M y b$ was modified in mouse embryonic stem cells by homologous recombination to include translational stop codons. Homozygous mutations were found to be lethal and analysis of the embryos identified severe anaemia due to failed erythropoiesis in the foetal liver (Mucenski et al., 1991). c$M y b$ expression was found to be required for the proper maturation of both myeloid and lymphoid lineages, except for megakaryocytes, indicating that functional c-Myb is required for normal haematopoiesis in vivo. Even though HSCs were found in the foetal liver of homozygous $c-M y b$ mutant mice, they failed to expand. This suggested that definitive haematopoiesis can be initiated in the absence of c-Myb, but that subsequent expansion is blocked (Sumner et al., 2000) (Clarke et al., 2000). In agreement with this, $c-M y b$ reduction to $5-10 \%$ of normal levels in murine bone marrow resulted in normal generation of haematopoietic progenitors but subsequent differentiation was found to be impaired. However, sufficient haematopoietic differentiation occurred to allow embryos to be born and reach adulthood 
(Emambokus et al., 2003). Other groups used mouse models with point mutations in $c-M y b$ that resulted in decreased $c-M y b$ activity or expression. Surprisingly, the results of these studies showed an increase in the number of haematopoietic progenitors and normal levels of mature myeloid cells (Carpinelli et al., 2004) (Sandberg et al., 2005) (Metcalf et al., 2005) (Greig et al., 2008). However, these models had some limitations. Thus, later on $c-M y b$ was conditionally depleted in murine bone marrow. In this new model, in contrast to previous results, $c-M y b$ depletion resulted in a reduced number of HSCs, abolishment of self-renewal and impaired development of the distinct haematopoietic lineages. Therefore, this model suggested an essential role of $c-M y b$ in the maintenance of HSCs and in adult haematopoiesis (Lieu and Reddy, 2009).

Aberrant $c-M Y B$ expression has been linked to haematopoietic malignancies in mice and humans. Several studies have shown increased $c-M Y B$ expression levels in myeloid and lymphoid leukaemias (Westin et al., 1982). However, dysregulation of $c-M Y B$ expression is normally indirect, as few direct genomic alterations have been described. In T-cell leukaemia, $c-M Y B$ translocations and duplications that contribute to the transformed phenotype have been found (Lahortiga et al., 2007) (Clappier et al., 2007) (O'Neil et al., 2007). Moreover, Mansour et al. also described mutations that introduce new c-MYB binding sites in non-coding genomic regions, which generate a super enhancer that activates the TAL1 oncogene in Tlymphoblastic leukaemia (Mansour et al., 2014). In AML, these genomic aberrations are less frequent but some studies also described a gain of the $c-M Y B$ locus in a rare type of AML (Murati et al., 2009), and an uncommon c-MYB-GATA-1 fusion 
oncogene (Belloni et al., 2011) (Quelen et al., 2011). Together, these finding support the idea that $c-M Y B$ expression is important for leukaemia initiation.

Indirect $c-M Y B$ upregulation is more common than genomic alterations in leukaemias and elevated $c-M Y B$ expression has been shown to be essential for disease maintenance. Numerous studies have provided additional evidence for the leukaemogenic potential of c-MYB by using in vitro cell systems. Enforced $c-M y b$ expression was found to block differentiation of murine erythroleukaemia and myeloblastic leukaemia cell lines (Clarke et al., 1988) (Selvakumaran et al., 1992). Moreover, exposure of different human AML cell lines to a synthetic antisense oligomer complementary to $c-M Y B$ mRNA resulted in a marked reduction in cell proliferation. Interestingly, the most differentiated cell lines were the most affected (Anfossi et al., 1989) (Gonda et al., 1989). Another study also used antisense oligonucleotides to inhibit $c-M Y B$ in normal haematopoietic progenitor cells and in myeloid leukaemia cells lines. Importantly, the results indicated a differential sensitivity to c-MYB inhibition, as normal progenitor cells survived at doses of $c$ MYB inhibition that were cytotoxic for leukaemic cells (Calabretta et al., 1991).

A number of recurrent chromosomal translocations in AML, such as $A M L$ ETO, PML-RAR $, E 2 A-H L F, B C R-A B L, M L L-A F 9$ and $M L L-E N L$, have been found to increase $c-M Y B$ expression or require $c-M Y B$ to induce transformation (Pattabiraman and Gonda, 2013) (Uttarkar et al., 2017). In particular, the induction by the $M L L$ fusion oncogenes has been well studied. In 2006, Hess and colleagues used a conditional mouse model of $M L L-E N L$ induced leukaemia and found that the 
fusion oncogene indirectly induced $c-M y b$ expression through HoxA9 and Meisl. Remarkably, $c-M y b$ depletion inhibited $M L L-E N L$ mediated transformation even when the level of $c-M y b$ knockdown was just around half of the normal level (Hess et al., 2006) (Pattabiraman and Gonda, 2013). A subsequent study used a mouse model of $M L L$-induced AML and found that $c-M Y B$, among other genes, is essential for the maintenance of the LSCs, and can induce transformation in a HoxA9/Meis1 independent manner (Somervaille et al., 2009). Similarly, a study led by our group used a conditional murine model of MLL-induced AML and $c-M y b$ was also identified as a downstream transcriptional target of the fusion proteins MLL-ENL and MLL-AF9 (Walf-Vorderwülbecke, 2009). In line with this, Zuber et al. also used a mouse model of $M L L$ rearranged AML and showed that $M L L-A F 9$ maintains the leukaemic phenotype by enforcing a $c-M y b$ genetic signature linked to self-renewal. Notably, their experiments showed that in vivo $c-M y b$ depletion supressed leukaemia progression without affecting normal myelopoiesis (Zuber et al., 2011). Moreover, a different study found that c-MYB drives $M L L$-induced leukaemias by forming a complex with MLL and Menin to contribute to the chromatin changes induced by the MLL-fusion proteins (Jin et al., 2010).

In summary, the studies described here determine the essential role of $c-M Y B$ in maintaining the leukaemic phenotype and describe the different ways in which leukaemic cells achieve high levels of $c-M Y B$ expression. Importantly, some of these studies also suggest that there may be therapeutic window in which leukaemic cells can be targeted without having major detrimental effects on normal myelopoiesis. 


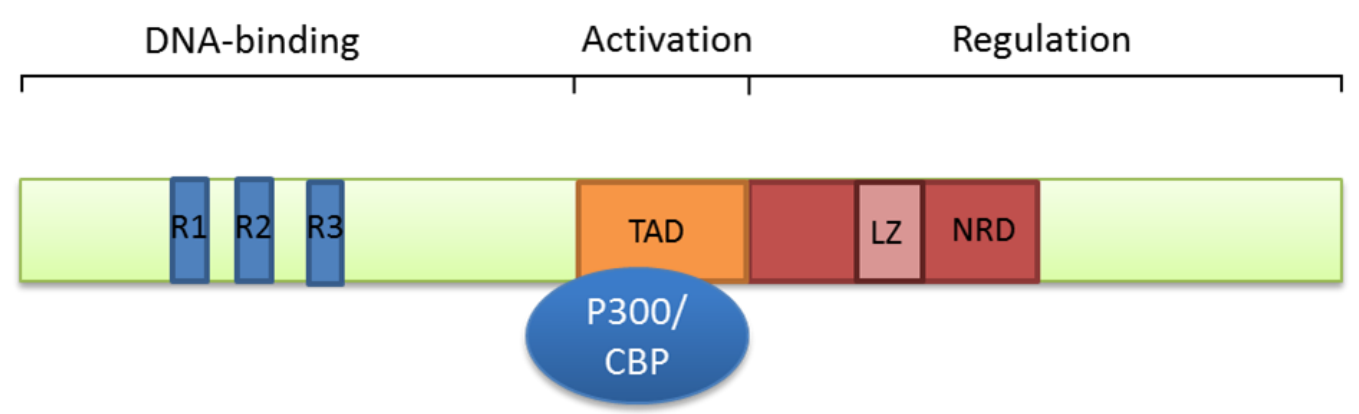

Figure 3. Schematic showing the c-MYB protein domains.

The c-MYB protein has a DNA binding domain at the $\mathrm{N}$-terminal region of the protein, a transactivation domain (TAD) to activate gene transcription at the center and a regulatory region at the c-domain of the protein. Some co-activators, such as the HAT p300/CBP, bind to the TAD of c-MYB. Adapted from (Uttarkar et al., 2017) and (Greig et al., 2008). R1, R2 and R3 represent regions of the DNA-binding domain; LZ, Leucine Zipper; NRD, Negative Regulatory Domain. 


\section{7 c-MYC in normal and aberrant haematopoiesis}

c-MYC is a transcription factor that regulates several critical cellular pathways, and whose expression has been reported to be deregulated in over $70 \%$ of all types of cancer (Dang, 2012). Its direct oncogenic activation can occur through gene amplification, chromosomal translocations, mutations or epigenetic deregulation. Moreover, many upstream pathways that activate $c-M Y C$ expression or that block its degradation have also been described (Gnanaprakasam and Wang, 2017). In haematological cancers, a chromosomal translocation that results in $c-M Y C$ activation is very common in Burkitt's lymphoma (Pelengaris et al., 2002). However, even though $c-M Y C$ deregulation has been generally linked to lymphoid malignancies, there is increasing evidence that it is also deregulated and plays an important role in myeloid leukaemias. In fact, $c-M Y C$ was initially discovered as a viral oncogene that produced myeloid neoplasms in chickens (myelocytomatosis), and the gene was named after this tumour (Delgado et al., 2013).

$c-M Y C$ encodes a basic helix-loop-helix leucine zipper (bHLH-Zip) transcription factor which forms part of a family of proteins comprising c-MYC, NMYC and L-MYC (Blackwood and Eisenman, 1991). It controls an extensive range of cellular functions, including cell growth, proliferation, differentiation and apoptosis. Most of its functions depend upon forming heterodimeric complexes with the MYC-Associated Factor X (MAX) protein. The complex formed by c-MYC and MAX binds to consensus sequences on the genome, known as Enhancer sequences (E-boxes), to activate gene expression through recruitment of chromatin remodelling 
co-factors (Blackwell et al., 1990) (Amati et al., 1993) (Poole and van Riggelen, 2017). Moreover, c-MYC/MAX complexes can bind to the MIZ-1 transcription factor to repress gene expression (Schneider et al., 1997) (Dang, 2012). c-MYC dimerises with MAX and MIZ-1 through the bHLH-Zip domain found on the Cterminal region of the protein, which also serves as a sequence specific DNA binding domain. The N-terminal region contains two conserved domains (c-MYC boxes I and II) which are essential for the transactivation of its target genes. Some cofactors, such as the ATPases Reptin and Pontin, have been found to interact with c-MYC through these domains (Figure 4) (Pelengaris et al., 2002) (Luscher and Larsson, 1999) (Wood et al., 2000).

In physiological conditions, $c-M y c$ is expressed during embryogenesis and has an essential role during development, as its ablation in embryonic stem cells resulted in embryonic lethality of homozygous mouse embryos between days 9.5 and 10.5. Moreover, the embryos displayed hematopoietic, placental and vascular defects (Davis et al., 1993) (Murphy et al., 2005). c-Myc expression is highly correlated with cell proliferation and thus, in adults it is highly expressed in dividing tissues. When cells enter mitotic arrest or start differentiating, $c-M y c$ transcript levels decline (Schmid et al., 1989). Several studies have shown that its effects on cell proliferation are in part due to the ability of c-MYC to activate or repress genes important for cell cycle progression, such as cyclin-dependent kinases (CDK) (Pelengaris et al., 2002). However, the effects of c-Myc on tissue growth are not only related to its function in regulating cell cycle, but also to its role in regulating cell size and protein synthesis (Johnston et al., 1999) (Iritani and Eisenman, 1999) (Schuhmacher et al., 1999). 
As previously mentioned, c-MYC regulates many cellular pathways. For this reason, its expression in physiological conditions is tightly regulated. Both mRNA and protein levels of c-MYC were found to have an extremely short half-life (Dani et al., 1984) (Hann and Eisenman, 1984), although to be constantly expressed during cell cycle (Hann et al., 1985) (Thompson et al., 1985). In fact, c-MYC expression has been found to be controlled by multiple signals and factors which tightly regulate the precise time and the amount of c-MYC transcription (Vervoorts et al., 2006). Moreover, posttranslational modifications such as phosphorylation, acetylation and ubiquitylation, which affect the stability of the protein, have also been described (Vervoorts et al., 2006). This regulation, however, cannot take place in tumour cells, as different mechanisms ensure enhanced levels of $c-M Y C$ expression (Pelengaris et al., 2002).

There has been a debate over how c-MYC regulates gene transcription. It has been found to recruit several co-factors which modulate gene expression by modifying the structure of the chromatin. For example, among other chromatin modulators, several HATs have been described that interact with c-MYC to activate gene expression, including the hGCN5 HAT via the adaptor protein TRRAP (McMahon et al., 2000); the CBP/p300 HAT cofactors (Vervoorts et al., 2003); and the HAT TIP60, together with other complex components such as p400 and Reptin/Pontin (Frank et al., 2003). Moreover, c-MYC was also found to interact with the pause-release factor $\mathrm{P}-\mathrm{TEFb}$, which promotes transcription elongation by phosphorylating RNA Polymerase II and other elongation factors. In fact, research in 
ES cells showed that c-MYC activates gene transcription by releasing paused Polymerase II from a large number of gene promoters in conjunction with $\mathrm{P}-\mathrm{TEFb}$ (Rahl et al., 2010). In line with this, later on c-MYC was described as a general amplifier that generally increases transcription of all genes expressed within a cell (Nie et al., 2012). Therefore, in physiological conditions, c-Myc transcriptional activation is related to its ability to accelerate transcription, rather than by activating particular group of genes (Wolf et al., 2015). Even though c-MYC was also described to function as a general amplifier in lymphoma cells expressing an inducible tetracycline (Tet)-repressible $M Y C$ transgene (Lin et al., 2012), later on it was shown that this not the case in cancer cells that express supraphysiological levels of c-MYC. A study that used human tumour cells to study c-MYC function found that aberrant highly expressed c-MYC binds with increased affinity to promoters which have non-consensus E-boxes, which are low-affinity c-MYC-binding sites. This generates a defined tumour-specific c-MYC expression profile in cancer cells, which differs from normal quiescent and dividing cells (Walz et al., 2014).

$c-M y c$ has been found to be expressed in foetal and adult HSCs and to have an essential role during normal myelopoiesis. Actually, one of the first biological effects described for $c-M y c$ was the inhibition of myeloid differentiation, as constitutive $c-M y c$ expression in a murine erythroleukaemia cell line blocked erythroid differentiation (Dmitrovsky et al., 1986) (Prochownik and Kukowska, 1986) (Coppola and Cole, 1986). Later on, another study obtained a similar result with a human chronic myeloid leukaemia cell line, and found that $c-M Y C$ levels declined when cells were forced to differentiate, while MAX levels remained 
unchanged (Delgado et al., 1995). Similarly, enforced expression of the $c-M Y C$ viral homolog $v-M Y C$ blocked macrophage differentiation of the human monoblastic cell line U-937 (Larsson et al., 1988) (Bahram et al., 1999). Moreover, a different study showed that, apart from inhibiting terminal myeloid differentiation, aberrant levels of $c-M y c$ expression also induced a p53 independent apoptosis response in normal murine myeloid cells and in a murine myeloid leukemic cell line (Amanullah et al., 2000). This apoptotic response was found to be mediated by premature recruitment of the cell death Fas/CD95 pathway (Amanullah et al., 2002). Furthermore, $c$-Myc was also found to be an important regulator of HSC homeostasis. Conditional $c-M y c$ deletion in the bone marrow of a mouse model led to accumulation of HSCs due to their inability to differentiate and, consequently, the mice developed severe anaemia. In contrast, $c-M y c$ overexpressing HSCs lost their ability to self-renew, most probably because of premature differentiation. Interestingly, these effects appeared to be dependent on the stem cell niche (Wilson et al., 2004). In a different study, conditional $c-M y c$ depletion in the myeloid compartment of mice resulted in severe thrombocytosis and decreased levels of neutrophils and monocytes, with an increase in megakaryocytes. This suggested that c-MYC has an opposite role in megakaryocytic versus monocytic and erythroid lineages (Guo et al., 2009). Collectively, these experiments showed that c-MYC has an essential role in normal haematopoiesis and myelopoiesis.

When $c-M Y C$ is deregulated in hematopoietic cells, the balance between differentiation and proliferation is lost, which can lead to leukaemia. Indeed, several studies have shown that $c-M y c$ enforced expression in bone marrow cells results in 
AML (Delgado et al., 2013). In 2005, retroviral gene transfer of $c$-Myc in the bone marrow cells of mice in the absence of antiapoptotic mutations resulted in AML. When $c-M y c$ was co-expressed with altered antiapoptotic pathways, the mice developed both myeloid and lymphoid leukaemias (Luo et al., 2005). Using another mouse model of $c-M y c$ induced leukaemogenesis, it was shown that all different members of the BCL anti-apoptotic family collaborate with c-MYC. Co-expression of $c-M y c$ and the different members of the BCL family resulted in an increase in the penetrance and a decrease in the latency of leukaemogenesis, in comparison to leukaemias induced by $c-M y c$ alone (Beverly and Varmus, 2009). Further investigations also led to the conclusion that the BCL family member MCL1 plays an important role in $c-M y c$ induced myeloid transformation (Xiang et al., 2010). Besides c-MYC, the related protein N-MYC was also found to rapidly induce clonal and transplantable AML in vivo (Kawagoe et al., 2007). Thus, overall these studies suggested that deregulated $c-M Y C$ expression, together with altered antiapoptotic pathways, can result in aggressive AML.

Other data also indicated the central role of $c-M Y C$ in myeloid tumorigenesis. For example, chromosomal translocations commonly present in AML, such as $A M L$ ETO, PML-RAR $\alpha$ or PLZF-RAR , were found to indirectly induce high levels of $c$ MYC expression (Muller-Tidow et al., 2004). Furthermore, the fusion oncogene $M L L-E N L$ was found to require $c-M Y C$ expression to induce a reversible differentiation arrest of a myelomonocytic precursor population. This arrest was counteracted by enforced expression of a c-MYC dominant negative mutant (Schreiner et al., 2001). Besides, a previous study in our laboratory also showed that 
immortalisation of murine progenitor cells with the $M L L$ fusion oncogenes leads to increased $c-M y c$ expression (Walf-Vorderwülbecke, 2009). A recent study also described increased $c-M y c$ levels in a mouse model of $M L L$ fusion-induced leukaemia and found that high $c-M Y C$ expression correlated with resistance to Bromodomain-containing protein 4 (BRD4) inhibitors, which normally inhibit leukaemia proliferation (Chen et al., 2016). Moreover, $c-M Y C$ (or $N-M Y C$ ) overexpression or amplification has been described in several other studies in different myeloid malignancies (Delgado et al., 2013), and its expression has been associated with poor outcome in clinical studies (Falantes et al., 2015). Collectively, these studies indicate that $c-M Y C$ deregulation is common in leukaemia and that $\mathrm{c}$ MYC upregulation plays an essential role in the development and maintenance of this malignancy. 


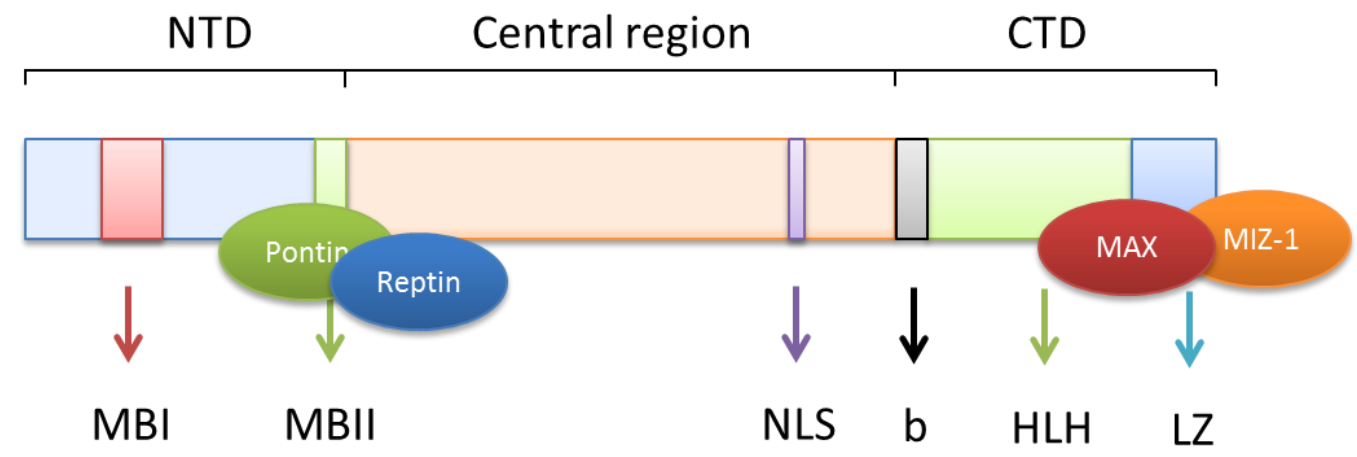

Figure 4. Schematic showing the functional domains of c-MYC.

Diagram depicting the different domains of c-MYC, and the regions where several cofactors (Reptin, Pontin, MIZ-1 and MAX) have been found to interact with cMYC. Adapted from (Pelengaris et al., 2002). MBI and MBII, c-MYC Boxes I and II; NTD, N-Terminal Domain; NLS, Nuclear Localisation Signal; b, basic; HLH, Helix-Loop-Helix; LZ, Leucine Zipper; CTD, C-Terminal Domain. 


\subsection{The ATPases Reptin (RUVBL2) and Pontin (RUVBL1)}

Reptin (also called RUVBL2, Tip48, Tip49b, RVB2, ECP51, TIH2 and TAP54 $\beta$ ) was first identified as a protein forming a complex with the paralogous protein Pontin (also called RUVBL1, Tip49, Tip49a, RVB1, ECP54, NMP238, TIH1 and TAP54 $\alpha$ ). Both Reptin and Pontin are highly conserved eukaryotic proteins that belong to the $\mathrm{AAA}^{+}$(ATPase associated with diverse cellular activities) superfamily of ATPases (Nano and Houry, 2013). They were discovered independently in a number of different species and were found to be essential for the viability of yeast, Drosophila melanogaster, C. elegans, Xenopus laevis and mice (Bauer et al., 1998) (Qiu et al., 1998) (Salzer et al., 1999) (Kanemaki et al., 1997) (Huber et al., 2008) (Bereshchenko et al., 2012). Reptin and Pontin were isolated from human cells for the first time in the late '90s (Kanemaki et al., 1997) (Kanemaki et al., 1999) and have been found to participate in multiple cellular functions, including DNA damage repair (Jha and Dutta, 2009); cellular transformation (Wood et al., 2000); telomere complex assembly (Venteicher et al., 2008); and transcriptional regulation through chromatin remodelling and by interacting with transcription factors (Jonsson et al., 2004) (Qi et al., 2006) (Kim et al., 2005) (Diop et al., 2008) (Tarangelo et al., 2015) (Figure 5). Even though most of their functions are carried out in the nucleus, Pontin was also found to be in the cytosol of human cervical cancer cells (Holzmann et al., 1998) and of human erythrocytes (Salzer et al., 1999). Other publications also found partial cytoplasmic localisation of Pontin or Reptin in different cell types, including monocytes (Grigoletto et al., 2011), and cytoplasmic expression of Reptin and Pontin in RCC and HCC was correlated with poor prognosis (Mao and Houry, 2017). More recently, an isoform of Reptin was also found localised in the mitochondria of 
leukaemic cells (Liyanage et al., 2017). In the nucleus, Reptin and Pontin perform many of their functions by forming part of large multi-subunit complexes together. However, some independent and antagonistic activities for Reptin and Pontin have also been described (Gallant, 2007).

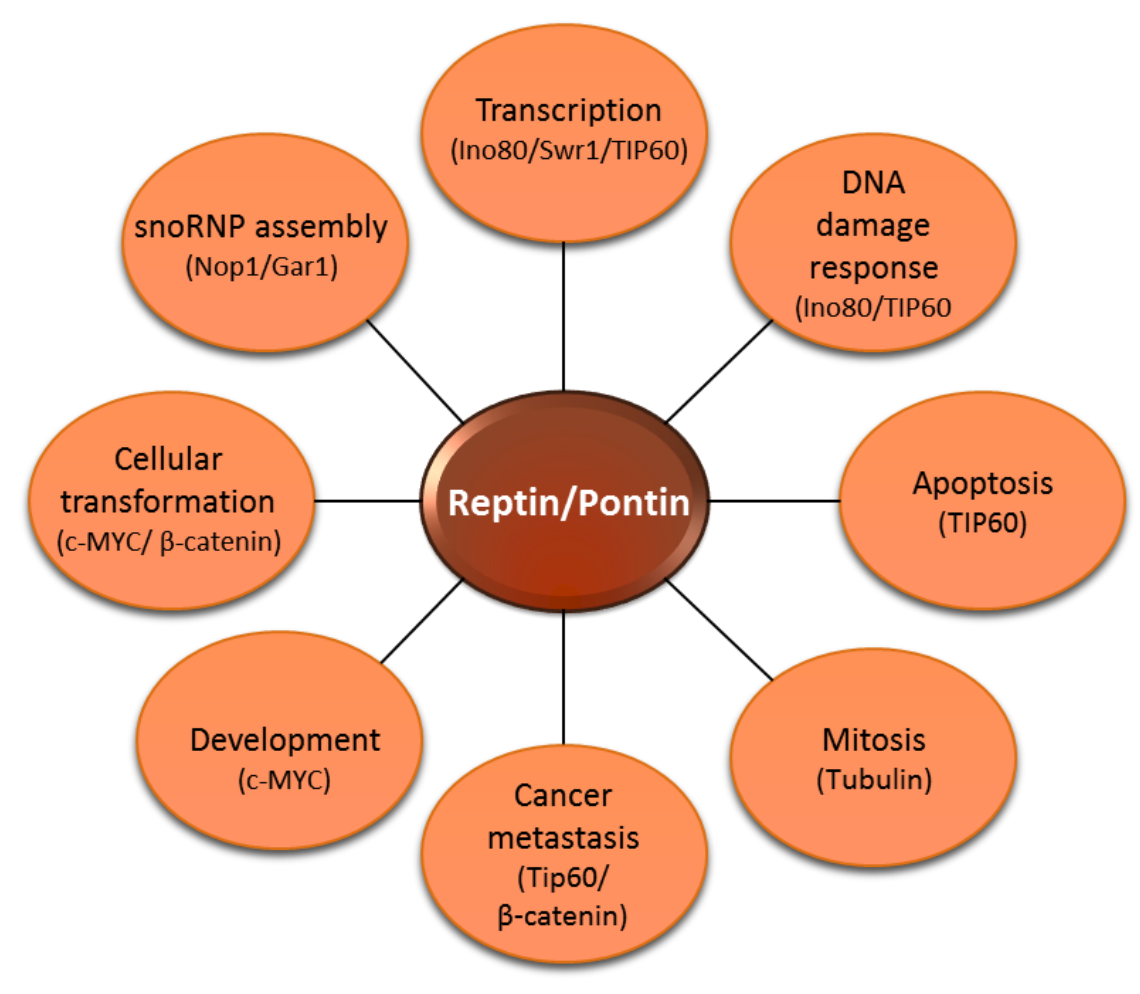

Figure 5. Reptin and Pontin regulate several cellular pathways.

Diagram showing the multiple cellular pathways that Reptin and Pontin have been described to regulate. Adapted from (Jha and Dutta, 2009). 


\subsubsection{Reptin and Pontin structure}

Proteins belonging to the $\mathrm{AAA}^{+}$superfamily of ATPases normally form heterohexameric ring structures composed of monomers. Reptin and Pontin monomers are characterized by the presence of Walker A and Walker B sequences, necessary for ATP binding and hydrolysis, respectively (Walker et al., 1982). For Reptin and Pontin, these sequences are included in their N-terminal Domain I. This is their core domain and is interrupted by the insertion domain, or Domain II. Interestingly, this insertion domain (Domain II) is not present in bacterial RuvB (Matias et al., 2006) (Niewiarowski et al., 2010). Additionally, there is a C-terminal Domain III, which is necessary to cap the nucleotide binding pocket in Domain I (Figure 6). Reptin and Pontin also contain an arginine finger and two sensor domains to sense whether the protein is bound to ADP or ATP (Nano and Houry, 2013). The ATPase pocket is formed by the Walker A and Walker B motifs of one monomer, together with the arginine finger from the neighbouring monomer (Huen et al., 2010).

The crystal structure of the human Reptin and Pontin complex, with truncated insertion domains, was solved in 2011 and was found to be a double hexameric ring (dodecamer) with alternating Reptin and Pontin monomers (Figure 6) (Gorynia et al., 2011). Additional research indicated that Reptin and Pontin can also exist in other oligomeric states modulated by the insertion domain, and found some biochemical differences between Reptin and Pontin that could explain their independent activities. For example, Reptin was found to form oligomers in the presence of adenine nucleotides, whereas Pontin did not form them (Puri et al., 2007) (Niewiarowski et 
al., 2010). More recently, by using a biochemical approach, Queval et al. proposed a model of the interplay between Reptin and Pontin monomers and hexamer complexes. They proposed a multistep process in which Reptin/Pontin monomers are initially recruited to nucleosome-free regions of the DNA. Protruding unmodified or methylated histone 3 (H3) tails from adjoining nucleosomes stabilize the DNAprotein complexes, resulting in the formation of the hexameric rings and their dissociation from the DNA. The newly formed complexes can then re-load to the proximal nucleosomal core particle. Interestingly, the authors found that for every oligomeric state, Reptin and Pontin can interact with a different subset of partner proteins. Moreover, they also found that when $\mathrm{H} 3$ tails are acetylated, the interaction with Reptin and Pontin is lost and the hexamerization does not happen (Figure 7) (Queval et al., 2014).

The ATPase activity of Reptin and Pontin appears to be essential for many of their cellular functions, including those relevant for cancer progression. For example, an ATPase deficient mutant of Pontin impaired its function in c-MYC (Wood et al., 2000), E1A (Dugan et al., 2002) or $\beta$-catenin (Feng et al., 2003) mediated oncogenic transformation. Moreover, a previous study in our laboratory found that the ATPase deficient mutant of Reptin induced apoptosis and abolished the clonogenic potential of AML cells in vitro (Osaki et al., 2013), and impaired leukaemia progression of mouse transplantations in vivo (PhD thesis, Zhao, 2013). Similarly, expression of this Reptin mutant in hepatocellular carcinoma cells also resulted in increased apoptosis and reduced proliferation in vitro (Grigoletto et al., 2013). However, in some studies certain functions were retained after mutating the ATPase domain. For example, the 
ATPase mutant of Pontin retained its capacity to bind c-MYC (Wood et al., 2000) and the ATPase mutant of Reptin retained its ability to repress the transcriptional activity of ATF2 (Cho et al., 2001). Moreover, the yeast Walker B mutant was found to retain the ability to recruit the INO80 subunit Arp5p to the complex, which maintained its chromatin remodelling function (Jonsson et al., 2004). Furthermore, overexpression of Walker A and B mutants in Xenopus laevis embryos resulted in the same phenotype as wild type overexpression of Reptin and Pontin (Etard et al., 2005). Thus, all of these data indicate that Reptin and Pontin have both ATPase dependent and ATPase independent functions (Grigoletto et al., 2011).

Reptin and Pontin have also been proposed to have ATP dependent helicase activities. However, this has been controversial. Whereas some groups detected the helicase activity of Reptin and Pontin in yeast and mammals cells (Gribun et al., 2008) (Kanemaki et al., 1999) (Makino et al., 1999), most publications were unable to detect it (Puri et al., 2007) (Qiu et al., 1998) (Ikura et al., 2000) (Matias et al., 2006). It is possible that the differences seen are related to the use of different protocols and different methods to assay the enzymatic activity of the proteins (Grigoletto et al., 2011). 
A)

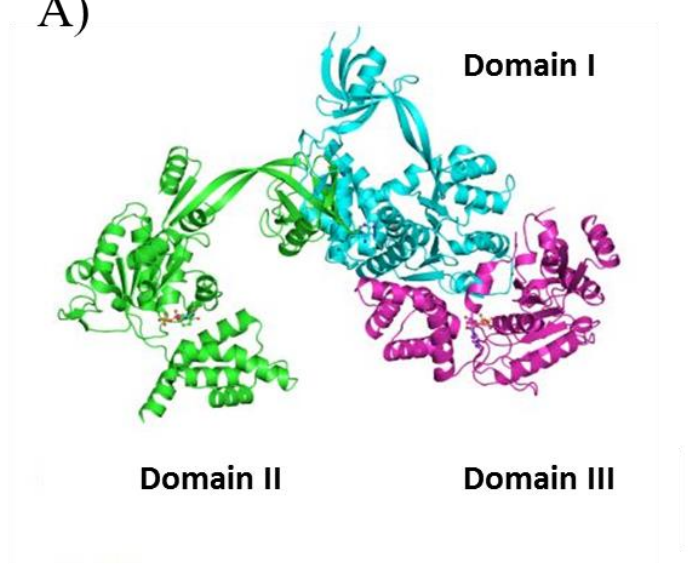

B)

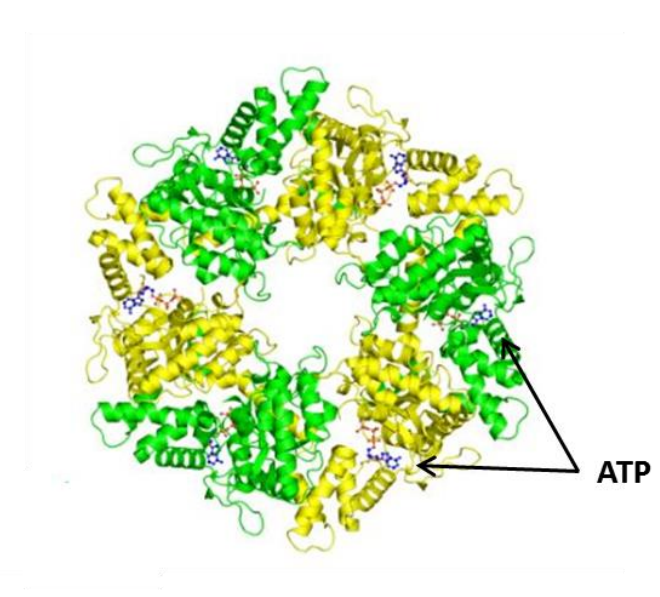

C)

\begin{tabular}{|l|l|l|l|l|}
\hline AAA+ domain $(\alpha \beta \alpha)(D I)$ & WA & Insertion domain (DII) & WB & AAA+ domain ( $\alpha$ ) (DIII) \\
\hline
\end{tabular}

\section{Figure 6. Structure and domains of Reptin and Pontin.}

(A) Ribbon diagram of the Pontin monomer (Protein Data bank identification number: 2c9o) showing its domain structure (Matias et al., 2006). (B) Ribbon diagrams of the Pontin and Reptin hexamer (alternating monomers shown in yellow and green), (Protein Data bank identification number: 2xsz) (Gorynia et al., 2011). Six molecules of adenosine-5'-triphosphate (ATP) are also shown and two of them are indicated with arrows. Two Reptin and Pontin hexamers can be joined to form a dodecamer. (C) Diagram showing the different domains of Reptin and Pontin. Domain I (DI) is pictured in red, while Domain II (DII) in blue and domain III (DIII), in dark green. Adapted from [(Lopez-Perrote et al., 2012) and (Carrie N. Arnold et al., 2006)]. WA = Walker A domain; $\mathrm{WB}=$ Walker $\mathrm{B}$ domain. 


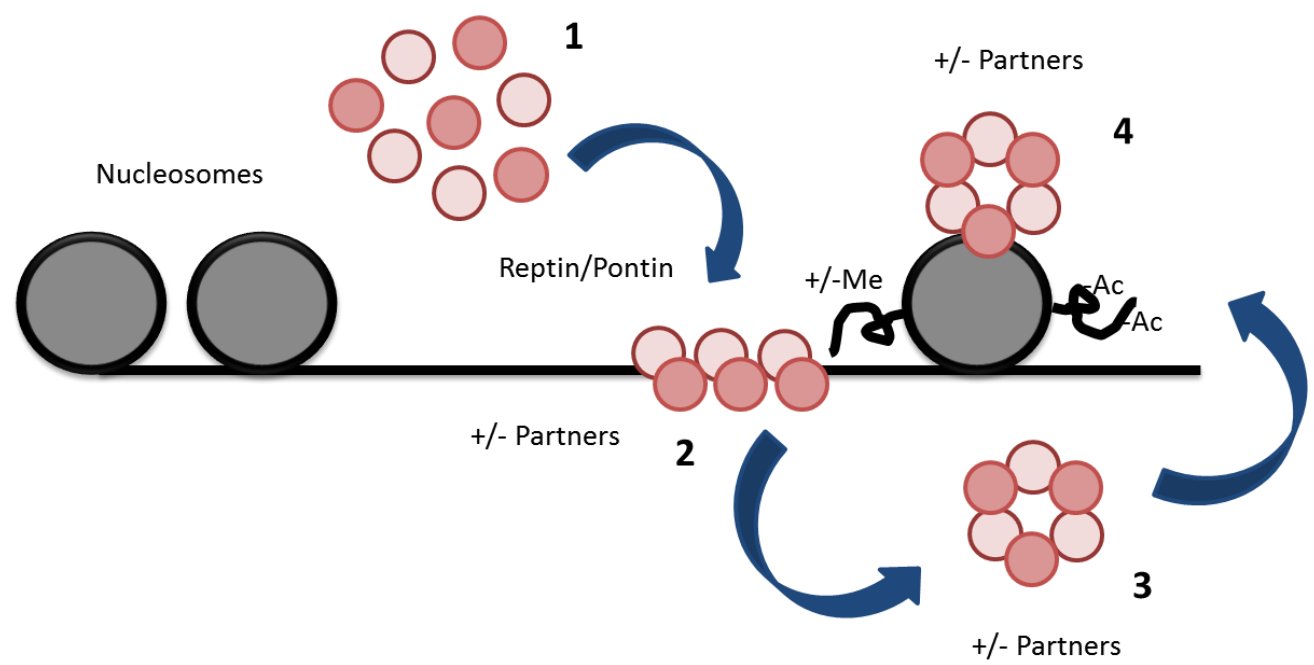

Figure 7. Model proposed to explain the interplay between Reptin, Pontin and chromatin.

This model published by Queval et al. proposes that Reptin and Pontin monomers (1) interact with nucleosome-free DNA, where they form a complex with unmodified or methylated $\mathrm{H} 3$ tails from neighbouring nucleosomes (2). Interaction with $\mathrm{H} 3$ tails induces hexamerisation and dissociation from the DNA (3). The Reptin/Pontin hexamers can bind to nucleosomes again (4). At every stage, Reptin and Pontin can associate with different proteins. Adapted from (Queval et al., 2014). Me, methylation; Ac, Acetylation. 


\subsubsection{Regulation of expression of Reptin and Pontin}

Reptin and Pontin proteins are expressed ubiquitously, although higher expression levels have been detected in thymus and testis. Even though their regulation is still not very well studied, there is evidence that their expression is tightly regulated, which will be discussed below (Grigoletto et al., 2011).

As many of their functions are based on their dodecameric structure, composed of Reptin and Pontin monomers, it was not surprising to find that their protein expression is interdependent. Thus, depletion of Reptin resulted in codepletion of Pontin protein, and vice versa, in a number of different human cell types (Venteicher et al., 2008) (Haurie et al., 2009) (Izumi et al., 2010) (PhD thesis, Osaki, 2011). This observation was at the protein and not at the transcript level, and further experiments showed that this co-regulation effect could be delayed using a proteasome inhibitor, suggesting their co-depletion is mediated by proteasomal degradation (Haurie et al., 2009). However, this co-depletion was not observed in the studies made by Baek's group, also in human cells (Kim et al., 2005) (Lee et al., 2010). Consistent with this tight protein regulation, overexpression of a tagged version of Reptin or Pontin in yeast, or an HA-tagged Reptin in human hepatocellular carcinoma cells, resulted in decreased expression of the endogenous protein (Radovic et al., 2007) (Rousseau et al., 2007).

There is little information on the transcriptional regulation of Reptin and Pontin, although some reports suggested a possible regulation through c-MYC. 
Pontin and Reptin mRNA and protein levels were reduced in a rat fibroblast cell line depleted of c-MYC, and were restored after re-expression of c-MYC or N-MYC (Wood et al., 2000). In line with this, Reptin mRNA was also found to be induced by c-MYC in human umbilical vein endothelial cells (Menssen and Hermeking, 2002). Moreover, c-MYC binding sites were found in the promoters of both Reptin and Pontin, suggesting a direct regulation by c-MYC (Zeller et al., 2006) (Fan et al., 2010). However, no correlation was found between $c$-MYC and Reptin transcript levels in a series of hepatocellular carcinoma samples (Rousseau et al., 2007). Furthermore, in a previous study from our group, $c-M Y C$ silencing in the human AML cell line THP-1 only led to a small reduction of Reptin transcript levels. In contrast, $c-M Y B$ silencing in the same cell line resulted in a marked decrease of Reptin mRNA levels (Osaki et al., 2013). This result was in agreement with another study in which, by using global gene expression data, Reptin (but not Pontin) was found to be a common target of both MLL-AF9 and c-MYB (Zuber et al., 2011). Consistent with this, Reptin expression was found to be decreased upon depletion of the $M L L$-fusion genes in mouse cells conditionally immortalized with MLL-AF9 or MLL-ENL (PhD thesis, Walf-Vorderwülbecke, 2009), and overexpressed upon immortalisation of human cord blood derived progenitor cells with the MLL-AF9 fusion gene (Osaki et al., 2013). Since c-MYB has been shown to be a downstream target of the MLL fusions in mouse and human cells, regulation of Reptin transcription by MLL fusion proteins was most likely indirect, and mediated via regulation of c-MYB expression (Osaki et al., 2013) (Hess et al., 2006) (Zuber et al., 2011). 
Reptin and Pontin have also been shown to be regulated by post-translational modifications. By using in vitro and in vivo sumoylation assays, Reptin was found to be sumoylated on Lysine 456, most likely by the sumoylation E2 conjugating enzyme UBC9 (Kim et al., 2006a). This modification increased nuclear localisation of Reptin and enhanced Reptin repressive activity on the promoter of the CD82/KAII gene. In contrast, desumoylation resulted in activation of the CD82/KAII gene, due to recruitment of the co-activator TIP60 (Kim et al., 2006a). The same group also described sumoylation of Pontin on a different lysine, which enhanced its effects on gene activation and increased proliferation of prostate cancer cells (Kim et al., 2007). Besides sumoylation, Reptin was also found to be methylated in response to hypoxia by the G9a enzyme, leading to a negative regulation of a subset of hypoxia target genes (Lee et al., 2010). Moreover, Reptin and Pontin have also been found to be phosphorylated and ubiquitylated, although the functional significance of these modifications is unclear (Grigoletto et al., 2011).

\subsubsection{Reptin and Pontin function: chaperone-like activity in the assembly of several $\underline{\text { complexes }}$}

Reptin and Pontin are components of several critical multi-subunit protein complexes, and there is increasing evidence suggesting a role for Reptin and Pontin as chaperone-like proteins facilitating the assembly of the different complexes (Nano and Houry, 2013). 
The INO80 complex is a chromatin remodelling complex that regulates gene transcription, DNA replication and repair of DNA double strand breaks by mobilizing nucleosomes along the DNA (Shen et al., 2000) (Jin et al., 2005). The complex includes both Reptin and Pontin and the INO80 protein, among other subunits. In yeast, the main ATPase activity of the complex was attributed to the INO80 protein, as a mutation of its ATPase activity was found to reduce the ATPase activity of the complex without affecting its composition (Nano and Houry, 2013). In contrast, depletion of Reptin and Pontin yeast homologs (Rvb1 and Rvb2) led to the loss of the Arp5p subunit and loss of the chromatin remodelling function of the complex, which suggested their role in the assembly of the INO80 complex (Jonsson et al., 2004). By studying the yeast INO80 complex, a recent publication proposed a model to explain how Rvbs assemble the multi-subunit complexes. In their model, binding of the INO80INS (a small insertion in the ATPase subunit of INO80) to the Domain II of Rvbs stabilizes this flexible domain (DII) and promotes formation of a metastable dodecamer. When other INO80 subunits bind to the dodecamer, ATP hydrolysis is stimulated and the dodecamer disassembles into single hexamers. This allows the assembly of the different INO80 subunits to fully assemble the INO80 complex (Zhou et al., 2017).

In addition to their role in the assembly of the INO80 complex, Reptin and Pontin were also found to be involved in the formation of the telomerase complex, a ribonuclear protein (RNP) complex (Nano and Houry, 2013). The main role of the telomerase complex, which is active in gametes, stem cells and tumour cells, is to add DNA repeats to the telomeres at the end of the chromosomes to avoid telomere 
attrition after every division and the subsequent senescent response (Martinez and Blasco, 2017). Reptin and Pontin were found to form part of the complex and their depletion led to the loss of some of the complex components and to a marked decrease in telomerase activity (Venteicher et al., 2008) (Osaki et al., 2013).

Reptin and Pontin have also been shown to be involved in the assembly of small nucleolar RNPs (snoRNPs). These are ribonucleolar protein complexes that contain small nucleolar RNAs (snoRNAs) and proteins. Reptin and Pontin have been found to weakly interact with proteins commonly associated with snoRNAs (NOP56, NOP58 and $15.5 \mathrm{~K}$ ) and the interaction has been found to be stimulated by ATP. Moreover, in both yeast and human cells, Reptin and Pontin were found to form part of the Rvb1-Rvb2-Tah1-Pih1 (R2TP) complex, which has been implicated in the assembly of snoRNP complexes. As correct assembly of the snoRNP complexes is necessary for its nucleolar localisation, and Reptin and Pontin depletion from the R2TP complex resulted in the mislocalisation of the snoRNP proteins, a role for Reptin/Pontin in the assembly of the complex has been suggested (McKeegan et al., 2009) (Nano and Houry, 2013). Moreover, this idea has further been reinforced recently by the finding that Reptin and Pontin interact with ZNHIT2 in the R2TP complex to regulate the protein composition of the U5 snoRNP complex (Cloutier et al., 2017). 


\subsubsection{Reptin and Pontin function: regulation of gene transcription}

Reptin and Pontin have been found to have a role in regulating gene transcription by forming part of several chromatin modifying complexes or by directly binding and modulating the function of several transcription factors (Gallant, 2007). With regard to their role in chromatin remodelling, Reptin and Pontin have been found to form part of and have essential roles in chromatin modifying complexes such as the INO80, TIP60, SRCAP/SWR1 and EP400 complexes (Nano and Houry, 2013) (Fuchs et al., 2001) (Jin et al., 2005) (Doyon et al., 2004).

The INO80 complex has already been introduced in the previous section of this chapter. As well as playing a role in the assembly of the INO80 complex, Reptin and Pontin are essential for its function, although not for its binding to the genome (Jonsson et al., 2004). Reptin was found to be necessary for the proper expression of many of the INO80 regulated genes. In yeast, a large overlap in the number of genes regulated by Rvbs and the INO80 complex was found, that was attributed to their function in the assembly of the complex, as Rvbs could not be found bound to the promoters of the INO80 target genes (Jonsson et al., 2004).

The SRCAP complex (called SWR1 in yeast) shares several subunits with the INO80 complex, including Reptin and Pontin. This complex remodels chromatin by catalysing the exchange of $\mathrm{H} 2 \mathrm{~A}-\mathrm{H} 2 \mathrm{~B}$ histone dimers in the nucleosomes by dimers containing the histone variant H2A.Z in mammals, or Htz1 in yeast (Nano and Houry, 2013). When Pontin was deleted by siRNA from the complex, the 
nucleosomal H2A.Z incorporation was found to be decreased. Since Reptin and Pontin are the only ATPases in the complex, and ATPase experiments confirmed their ATPase activity in the complex, the authors of the study proposed that the ATPase function of Reptin and Pontin is necessary for the exchange of the histone variant (Choi et al., 2009).

TIP60 is a chromatin remodelling complex that regulates gene transcription by acetylating histones at promoters to relax the chromatin and facilitate access to DNA. It can also directly acetylate proteins and has been found to have a role in the DNA-damage response (Jha and Dutta, 2009). A variant of the TIP60 complex is the EP400 complex, which also has HAT activity at promoters (Fuchs et al., 2001). Human Reptin and Pontin have been found to be components of both complexes. In fact, Pontin was found to be required for the acetylating function of the TIP60 complex during the DNA-damage response in human cervical cancer cells (Jha et al., 2008). Moreover, Pontin was found to regulate the expression of some target genes of $\beta$-catenin in conjunction with Tip60 and other components of the complex (Feng et al., 2003). Additionally, depletion of Reptin and Pontin, among other EP400 complex components such as EPC1 or DMAP1, resulted in the accumulation of the c-MYC protein in AML cells (Huang et al., 2014).

$\beta$-catenin is a transcription factor that forms part of the WNT signalling pathway. In dividing cells, the WTN ligand binds to the frizzled (FZD) receptor, which results in the accumulation of $\beta$-catenin and its translocation to the nucleus. $\beta$ catenin can then, in association with a member of the LEF/TCF family, activate the 
expression of necessary genes for cell proliferation and survival, such as c-MYC or cyclinD1 (McCubrey et al., 2014). In haematopoietic cells, the WNT signalling pathway has been shown to play an essential role in maintaining HSC fate and normal haematopoiesis, and dysregulation of this pathway leads to haematological diseases such as leukaemias (McCubrey et al., 2014). Therefore, it is interesting that Reptin and Pontin were found to interact with $\beta$-catenin and regulate its transcriptional activity. Surprisingly, using reporter assays, Reptin and Pontin were found to have opposing roles in this pathway. While Pontin was found to enhance $\beta$ catenin target gene transcription, Reptin was found to repress it (Bauer et al., 1998) (Bauer et al., 2000). Another example in which Reptin and Pontin were found to have antagonistic activities in conjunction with $\beta$-catenin was in the regulation of the metastasis suppressor $C D 82 / K A I 1$. In particular, Reptin was found to bind $\beta$-catenin to repress the expression of this gene in metastatic prostate cancer cells, while Pontin was found to activate the expression of the CD82/KAIlgene in non-metastatic cells, through interaction with TIP60 instead of $\beta$-catenin (Kim et al., 2005). In addition, Reptin and Pontin were found to bind the tumour suppressor Hint1, a co-regulator of the $\beta$-catenin pathway. The interaction with Hint 1 disrupted the formation of homo and hetero Reptin and Pontin complexes, which resulted in inhibition of $\beta$-catenin mediated transcription (Weiske and Huber, 2005).

Reptin and Pontin were also found to have antagonistic roles in the regulation of the Hox genes in Drosophila melanogaster. In particular, Reptin was found to be part of the PRC1 Polycomb group complex and repress expression of the Hox genes, while Pontin was found to interact with the Brahma complex/Trithorax group to act 
as a co-activator instead (Diop et al., 2008). Interestingly, Reptin and Pontin were also found to be part of the MLL1-WDR5 complex in human cells. The MLL1WDR5 complex regulates Hox gene expression through chromatin modifications. Although the exact role of Reptin and Pontin in the complex was not determined, it is possible that they are also involved in Hox gene regulation in human cells (Dou et $a l ., 2005)$.

Remarkably, Reptin and Pontin were also found to interact with an important transcription factor in cancer, the oncoprotein c-MYC (Wood et al., 2000). Both Reptin and Pontin were found to bind to the highly conserved Box II region in the Nterminal domain of c-MYC (Wood et al., 2000) (Bellosta et al., 2005). While both proteins were found to interact with c-MYC, the ATPase activity of Pontin was found to be essential for c-MYC-mediated oncogenic transformation of rat embryo fibroblasts transduced with the oncogene $H-R_{a s}{ }^{\mathrm{G} 12 \mathrm{~V}}$ (Wood et al., 2000). A study using Drosophila melanogaster as a model organism also found a crucial interaction of Pontin (and possibly Reptin) with c-MYC, which is important to control tissue growth in vivo during development. In particular, simultaneous Reptin and $c-M Y C$ downregulation in flies resulted in reduced viability in comparison to $c-M Y C$ mutant only flies, and the most characteristic feature of surviving flies was the presence of eye defects. Moreover, by using siRNA to deplete Reptin, Pontin or c-MYC expression in Drosophila cells, the authors found an overlap in some genes regulated by both Reptin/Pontin and c-MYC (Bellosta et al., 2005). Moreover, in a different study a dominant negative mutant of Pontin was found to promote c-MYC mediated apoptosis (Dugan et al., 2002). In addition, the TIP60 chromatin remodelling 
complex, which acetylates histones to modulate gene transcription and includes both Reptin and Pontin, was also found to be recruited by c-MYC to its target genes in vivo (Frank et al., 2003) and to co-regulate expression of c-MYC target genes in ES cells (Ravens et al., 2015). In line with this, Reptin and Pontin were identified to interact with the oncogenic protein MTBP to enhance c-MYC mediated transcription, proliferation and neoplastic transformation (Grieb et al., 2014). Reporter studies also showed that wild type Reptin and Pontin, but not their N-terminal mutants, can increase c-MYC/MIZ-1 repression of $p 21^{C I P 1}$ in Xenopus embryos (Etard et al., 2005) and $C / E B P \delta$ promoter activity in a mouse cell line (Si et al., 2010). Additionally, Reptin depletion resulted in the abrogation of c-MYC binding to the promoter of the human Telomerase Reverse Transcriptase (hTERT) gene, suggesting a role for Reptin in the transcriptional regulation of the telomerase complex components through c-MYC (Li et al., 2010).

Reptin and Pontin have also been shown to modulate the transcriptional response of another transcription factor, E2F1. In 2002, Pontin was reported to interact with the transactivation domain of E2F1. Moreover, when a dominant negative mutant of Pontin was overexpressed with E2F1, it was found to greatly potentiate the apoptotic phenotype normally induced by E2F1 (Dugan et al., 2002). E2F1 is a transcriptional activator repressed under physiological conditions in liver cells by Retinoblastoma $(\mathrm{Rb})$ proteins, to maintain them in a quiescent state. In hepatocellular carcinoma, extracellular mitogenic signals remove this transcriptional block and stimulate the transcriptional activity of E2F1, which promotes cell proliferation. Tarangelo et al. recently identified the mechanism by which Reptin and 
Pontin lead to a progressive amplification of E2F1 target gene expression in the late stages of hepatocellular carcinoma. In their model, E2F1 recruits Reptin and Pontin to low affinity promoter regions to incorporate the histone variant H2A.Z to the chromatin. This destabilises the chromatin, allowing the activation of the low affinity target genes of E2F1, which are important for cancer progression (Tarangelo et al., 2015).

In another study, Reptin and Pontin were found to modulate the function of HIF-1 $\alpha$, a transcription factor that mediates many responses to hypoxia. As previously mentioned, Reptin was found to be methylated in response to hypoxia by the G9a methyltransferase. This led to the recruitment of the histone deacetylase HDAC1 to the promoters of some of the HIF-1 $\alpha$ regulated genes and repression of this subset of genes. In contrast, Pontin was also found to be methylated but to act as an activator by recruiting the HAT p300 to HIF-1 $\alpha$ target genes (Lee et al., 2010).

Reptin and Pontin also regulate the DNA damage response, nutrient dependent signalling and non-sense mediated decoy (a mechanism to eliminate mRNAs containing premature translation-termination codons), through modulation of all proteins belonging to the family of Phosphatidylinositol 3-kinase-related protein kinases (PIKK), such as ATR and ATM (Grigoletto et al., 2011) (Brogna and Wen, 2009). Depletion of Reptin and Pontin resulted in a decrease of mRNA and protein levels of PIKK proteins. Moreover, their loss also resulted in a decrease of PIKK signalling after UV or $\gamma$ irradiation, as there was a reduction in the phosphorylation of downstream PIKK targets. While wild type Reptin and Pontin 
were able to rescue PIKK protein levels, the ATPase deficient mutant forms failed to do so (Izumi et al., 2010). Subsequent work from the same group showed that Reptin and Pontin form a complex with the chaperone HSP90 to regulate PIKK signalling (Izumi et al., 2012).

\subsubsection{Reptin and Pontin in Cancer}

As described in the previous section, Reptin and Pontin interact with and modulate the function of several transcription factors with essential roles in cancer. In relation to this, Reptin and Pontin expression has been described to be deregulated in several types of cancer.

In hepatocellular carcinoma, Reptin and Pontin were found to be overexpressed in comparison to normal liver and the overexpression was found to be associated with poor prognosis (Blanc et al., 2005) (Rousseau et al., 2007) (Haurie et al., 2009) (Iizuka et al., 2006). Moreover, the ATPase activity of Reptin was identified as necessary for its effects on the growth and viability of the tumour cells (Grigoletto et al., 2013). Additional research also identified the gene Meprin alpha (MeplA) as an important downstream target of Reptin in HCC, which regulates cell migration and invasion (Breig et al., 2017).

In renal cell carcinoma, overexpression of Reptin in the cytoplasm of the cells was found to be associated with poor differentiation of the tumours and poor prognosis (Ren et al., 2013). Reptin was also observed to be overexpressed in primary gastric cancer samples, and its inhibition in gastric cells led to growth arrest 
and a decrease of hTERT promoter activity and mRNA (Li et al., 2010). Overexpression of Reptin was also observed in a number of breast cancer samples in comparison to normal tissue of matching patients. In this study, Reptin was found to interact with Anterior gradient-2 (AGR2), a protein found to be associated with resistance to tamoxifen treatment in breast cancer patients. Reptin Walker A and B mutants were found to disrupt the interaction with AGR2 (Maslon et al., 2010). Pontin was found to be overexpressed in colon cancer cells, relative to paired normal tissue, and to promote expression of Cyclooxygenase-2 (COX-2), a protein know to have an essential role in the development of the disease (Carlson et al., 2003). The overexpression of Pontin in colon cancer was further examined by immunohistochemistry in a different study and the authors observed a stronger staining in the invasive margins of the tumours (Lauscher et al., 2007). Moreover, Reptin was also found to be overexpressed in colorectal carcinoma in comparison to normal mucosa (Milone et al., 2016)

Intriguingly, aberrant Reptin and Pontin expression has also been linked to leukaemia. Pontin was identified as a critical mediator of AML1-ETO expressing leukaemic cells. Pontin expression was found to be regulated by the AML1-ETO oncogene and its depletion inhibited the growth and self-renewal capacity of human AML1-ETO-expressing leukemic blood cells (Breig et al., 2014). Moreover, a previous study in our laboratory showed that Reptin expression decreases upon loss of MLL-ENL and MLL-AF9 expression in conditionally immortalised mouse myeloid cells (PhD thesis, Walf-Vorderwülbecke, 2009). By depleting Reptin expression or overexpressing a Walker B mutant of Reptin in an MLL-AF9 
expressing human cell line, further work in our laboratory also found that Reptin is required for the proliferation, survival and clonogenic potential of $M L L$ rearranged AML. Regulation of Reptin gene expression was found to be indirectly controlled by MLL-fusion proteins, via the transcription factor c-MYB. Interestingly, Reptin expression was also found to be required for the growth of other non-MLL rearranged AML cell lines (Osaki et al., 2013). Further studies in our group assessed the effect of Reptin depletion on leukaemia progression in vivo. Clones from mouse cells immortalised with the $M L L-E N L$ fusion gene and transduced with an inducible shRNA, to deplete Reptin expression, were transplanted into mice. Reptin depletion in the established leukaemias impaired leukaemia progression in vivo. Similar results were obtained when an ATPase mutant of Reptin was expressed in the transplanted leukaemic cells (PhD thesis, Zhao, 2013). In the same study, the effect of the ATPase mutant of Reptin on normal myeloid development was also examined. Normal mouse progenitor cells were transduced with the Walker B mutant of Reptin and the number of myeloid colonies formed counted. Remarkably, the results indicated no significant difference in the number of colonies between control cells and cells expressing the Reptin mutant (PhD thesis, Zhao, 2013). 


\section{PROJECT AIM}

The aim of this $\mathrm{PhD}$ project is to investigate further the role of Reptin in human acute myeloid leukaemia (AML). Therefore, the main objectives of the project are:

1. To examine the efficacy of targeting Reptin in eliminating established human leukaemia in vivo.

2. To identify the transcriptional pathways regulated by Reptin in human AML by generating global gene expression analysis after Reptin depletion.

3. To validate and further study the transcriptional pathways regulated by Reptin. 


\section{CHAPTER II - Materials and methods}

\subsection{Molecular Biology}

\subsubsection{Bacterial Transformation}

Sub-cloning or library efficiency $\mathrm{DH} 5 \alpha^{\mathrm{TM}}$ and One Shot ${ }^{\circledR}$ Stbl $3^{\mathrm{TM}}$ chemically competent cells (ThermoFisher Scientific) were thawed on ice and gently mixed with $3 \mu \mathrm{L}$ ligation product or $10-100 \mathrm{ng}$ plasmid DNA. Following 30 minutes incubation on ice, cells were heat-shocked for $20 \mathrm{sec}$ or 45 seconds at $42{ }^{\circ} \mathrm{C}$ and returned to ice for 2 min. After adding $300 \mu \mathrm{L}$ pre-warmed S.O.C. medium (New England Biolabs), cells were incubated in a shaker at $37^{\circ} \mathrm{C}$ for one hour at $225 \mathrm{rpm}$. The total volume or a dilution of each transformation was spread on a Luria broth (LB) agar plate (1.5g Bacto Agar [BD bioscience] per 100ml LB [1\% w/v Bacto Tryptone (BD Bioscience), $0.5 \%$ w/v Bacto Yeast Extract (BD Bioscience), 1\% w/v Sodium Chloride, (pH 7.0)]) supplemented with $100 \mu \mathrm{g} / \mathrm{ml}$ Ampicillin (Sigma-Aldrich). The plates were incubated overnight at $37{ }^{\circ} \mathrm{C}$.

\subsubsection{Plasmid DNA purification}

To purify small amounts of DNA, used for cloning, a single bacterial colony was picked and inoculated into $5 \mathrm{ml}$ LB broth (1\% w/v Bacto Tryptone [BD Bioscience], 0.5\% w/v Bacto Yeast Extract [BD Bioscience], $1 \%$ w/v Sodium Chloride, [pH7.0] ) containing $100 \mu \mathrm{g} / \mathrm{ml}$ Ampicillin for selection and incubated overnight at $37^{\circ} \mathrm{C}$ with shaking. Plasmid DNA was isolated from the bacterial cultures using the 
PureYield $^{\mathrm{TM}}$ Plasmid Miniprep System (Promega), according to the manufacturer's instructions.

For large quantities of plasmid DNA, used for transfections, a single bacterial colony was picked and inoculated into $5 \mathrm{ml} \mathrm{LB}$ broth containing $100 \mu \mathrm{g} / \mathrm{ml}$ Ampicillin for 6 hours at $37^{\circ} \mathrm{C}$ with shaking. This starter culture was then transferred into $300 \mathrm{ml} \mathrm{LB}$ broth containing $100 \mu \mathrm{g} / \mathrm{ml}$ Ampicillin and incubated for at least 16 hours at $37^{\circ} \mathrm{C}$ with shaking. Plasmid DNA was isolated from the bacterial cultures using Genopure Plasmid Maxi Kit (Roche) according to the manufacturer's instructions. The plasmid DNA concentration was determined by measuring the absorbance at $260 \mathrm{~nm}$ (A260) using a spectrophotometer (NanoDrop ND-1000, Labtech International). The ratio of absorbance at $260 \mathrm{~nm}$ to $280 \mathrm{~nm}\left(\mathrm{~A}_{260 / 280}\right)$ was used to assess the purity of DNA. A ratio of around 1.8 was considered pure DNA.

\subsubsection{Restriction enzyme digests}

Restriction enzymes (Thermo Scientific) were used to cleave double stranded DNA. The digests were set up according to the manufacturer's instructions. Briefly, DNA was digested with $0.3 \mu \mathrm{l}$ or $0.6 \mu \mathrm{l}$ of $10 \mathrm{U} / \mu \mathrm{l}$ restriction enzyme per $\mu \mathrm{g}$ of DNA, $10 \mathrm{x}$ restriction buffer $\left(10 \mathrm{mM}\right.$ Tris-HCL $\left(\mathrm{pH} 7.5\right.$ at $\left.37^{\circ} \mathrm{C}\right), 10 \mathrm{mM} \mathrm{MgCl}_{2}, 50 \mathrm{mM} \mathrm{NaCl}$, $0.1 \mathrm{mg} / \mathrm{ml} \mathrm{BSA}$ ) and the volume made up to $100 \mu \mathrm{l}$ with $\mathrm{H}_{2} \mathrm{O}$. The digested product was incubated for one to four hours at $37^{\circ} \mathrm{C}$ (depending on the enzyme) and subjected to electrophoresis on 1\% w/v Agarose gels (Agarose [Invitrogen], 1x TAE buffer [National diagnostics], 0.5\% w/v Ethidium Bromide [Sigma]). 


\subsubsection{Gel extraction}

QIAquick Gel Extration Kit (Qiagen) was used to extract and purify DNA from Agarose gels according to the manufacturer's instructions. The concentration of purified DNA was determined using the NanoDrop ND-1000.

\subsection{5 $\underline{\text { Ligation }}$}

For cloning, a DNA fragment and the vector were ligated. The molar ratio between the fragment and the vector varied from 1:1 to 5:1. For example, to calculate the required amount of insert for a 3:1 ligation, the following equation was used:

$n g$ insert required $=[(100 \mathrm{ng}$ vector $\cdot x \mathrm{~kb}$ insert $) / \mathrm{kb}$ vector $] \times 3$

Generally the ligation reaction was set up using $10 \mu \mathrm{l}$ of $2 \mathrm{x}$ rapid Ligation buffer (Promega) and 1 Weiss unit of T4 DNA ligase (Promega) in a total volume of $20 \mu$, made up with $\mathrm{H}_{2} \mathrm{O}$. The mixture was incubated at room temperature for 1-6 hours prior to use in bacterial transformation (refer to 2.1).

\subsubsection{Polymerase chain reaction (PCR)}

Template specific primers were designed and provided by Sigma-Aldrich. PCR was performed using GoTaq ${ }^{\circledR}$ DNA Polymerase (Promega). For the PCR reaction mix, $25 \mathrm{ng}$ insert was mixed with $10 \mathrm{x}$ reaction buffer, $0.25 \mu \mathrm{l}(1 \mathrm{U})$ Taq polymerase, 0.2 $\mathrm{mM}$ dNTP, $1.5 \mathrm{mM} \mathrm{MgCl} 2,5 \mu \mathrm{M} 75$ forward primer, $5 \mu \mathrm{M}$ reverse primer and $\mathrm{H}_{2} \mathrm{O}$ up to $50 \mu \mathrm{l}$ total volume. The PCR was performed using the cycle conditions illustrated in Table 5. 


\begin{tabular}{cccc}
\hline STEP & TEMPERATURE & TIME & NUMBER OF \\
Initial & $95^{\circ} \mathrm{C}$ & 2 minutes & 1 cycle \\
denaturation & & & \\
Denaturation & $95^{\circ} \mathrm{C}$ & 30 seconds & 30 cycles \\
Annealing & $55^{\text {to } 66}$ & 30 seconds & 30 cycles \\
Extension & $72^{\circ} \mathrm{C}$ & 1 minute/kbp & 30 cycles \\
\hline Final extension & $72^{\circ} \mathrm{C}$ & 5 minutes & 1 cycle \\
\hline
\end{tabular}

Table 5. PCR conditions used in this study.

\subsubsection{DNA constructs}

- Lentiviral gene overexpression:

Schematic diagrams of the lentiviral expression constructs used in this study for overexpression are shown in Figure 8. The pHRSIN CSGW was a kind gift from Y.Ikeda (Mayo Clinic, Rochester, MN). This vector was generated by removing one NOTI site from the SIN-CSGW vector. The vector was subsequently modified to replace the eGFP sequence with a PGK-IRES-eGFP from pMSCV-PGK-PUROIRES-eGFP vector. 
pHRSIN CGSW-PIG:

5'LTR $\psi$ RRE c 4 PPT SFFV PGK Puror - IRES GFP WRE 3'LTR AMPr

pHRSIN CGSW-PIG-D/N RUVBL2 (D299N) :

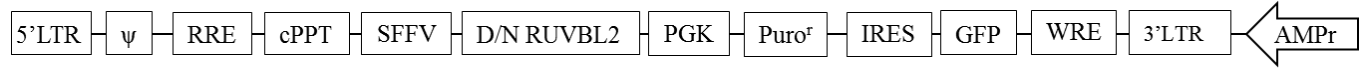

Figure 8. Lentiviral expression constructs used in this study.

LTR, long terminal repeat; $\psi$, viral packaging signal; RRE, Rev-Response Element; cPPT, Central Polypurine tract; SFFV, Spleen focus-forming virus promoter; PGK, murine phosphoglycerate kinase promoter; Puro $^{\mathrm{r}}$, puromycin resistance gene; IRES, Internal ribosome entry site; GFP, Green Fluorescent protein; WRE, Woodchuck response element; AMPr, Ampicillin resistance gene for bacterial selection.

\section{- Constitutive gene downregulation:}

The pGIPZ and MISSION pLKO.1 shRNA systems were used in this study to constitutively knockdown gene expression. The pGIPZ vector to deplete Reptin expression was obtained from $\mathrm{Dr}$ Lu Zhao (PhD thesis, Zhao, 2013); to deplete Pontin, from Dr Hikari Osaki (PhD thesis, Osaki, 2011); and to deplete $M T B P$, from the Open Biosystems GIPZ shRNAmir lentiviral library owned by UCL. The shRNA sequences included in the pGIPZ constructs used in this study are shown in Table 6.

The MISSION® pLKO.1 shRNA constructs to deplete Reptin, EPC1, EPC2, $c-M Y B$, and the scrambled control, were purchased from Sigma Aldrich or 
Dharmacon. Schematic diagrams of the pGIPZ and pLKO.1 constructs used in this study are shown in Figure 9. The shRNA sequences included in the MISSION® pLKO. 1 constructs used in this study are shown in Table 7.

\section{SFFV-GIPZ-GFP}

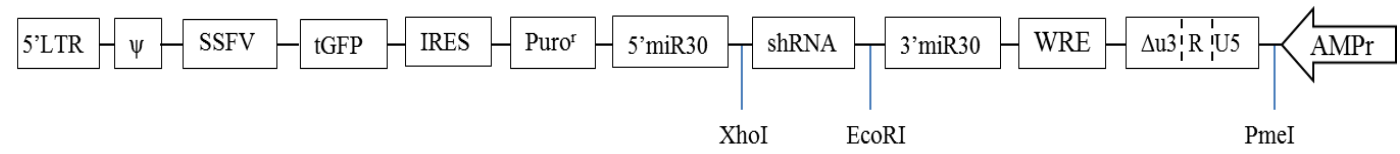

MISSION $^{\circledR}$ pLKO.1-puro U6

pUC ori $\mathrm{RSV} /$ ' $^{\prime} \mathrm{LTR}-\mathrm{RRE}-\mathrm{U} 6-\mathrm{shRNA} \mathrm{CPPT}$ PGK Puro $^{\mathrm{r}}$ SIN/3'LTR F1 ori

Figure 9. Lentiviral expression constructs used in this study to constitutively knockdown gene expression.

LTR, long terminal repeat; $\psi$, viral packaging signal; RRE, Rev-Response Element; cPPT, Central Polypurine tract; SFFV, Spleen focus-forming virus; PGK, murine phosphoglycerate kinase promoter; Puro $^{\mathrm{r}}$, puromycin resistance gene; IRES, Internal ribosome entry site; GFP, Green Fluorescent protein; WRE, Woodchuck response element; pUC ori, pUC origin of replication; RSV, respiratory syncytial virus; 3' selfinactivating long terminal repeat; F1 ori, F1 origin of replication; AMPr, Ampicillin resistance gene for bacterial selection. 


\begin{tabular}{|c|c|}
\hline GENE NAME & MATURE ANTISENSE SEQUENCE (5'-3') \\
\hline Scrambled (shSCR) & ATCTCGCTTGGGCGAGAGTAAG \\
\hline Reptin (shRep) & ACAGCGAGAAAGACACGAAGCA \\
\hline Pontin (shPon) & AAAGACAGAAATCACAGACAAA \\
\hline$M T B P(\# 1)$ & TTAGATCTCCGTTTCAATC \\
\hline $\operatorname{MTBP}(\# 2)$ & TACATTAACATAGCATCCC \\
\hline $\operatorname{MTBP}(\# 3)$ & TGAACATTAGCTAACTGTT \\
\hline $\operatorname{MTBP}(\# 4)$ & TAATTGAAGCACTAATGCT \\
\hline
\end{tabular}

Table 6. List of pGIPZ shRNA sequences used in this study.

\begin{tabular}{ccc}
\hline GENE NAME & CLONE ID & SEQUENCE \\
\hline Reptin & TRCN0000051566 & GCAGTACATGAAGGAGTACCA \\
Reptin & TRCN0000051563 & CGAGAAAGACACGAAGCAGAT \\
$\boldsymbol{E P C 1}$ & TRCN0000218988 & CAGCCAATGAAACCTACTTAT \\
$\boldsymbol{E P C 1}$ & TRCN0000229401 & TGGCGAGAAGAGGGATAATAT \\
$\boldsymbol{E P C 2}$ & TRCN0000363301 & CGAAGATGATTACCTTATTAA \\
$\boldsymbol{E P C 2}$ & TRCN0000363358 & GACATTGCCTGTGATCAATAA \\
$\boldsymbol{E P C 2}$ & TRCN0000359790 & GGTCATAATGGACCGAATATC \\
$\boldsymbol{c}$ - $\boldsymbol{M Y \boldsymbol { B }}$ & TRCN0000009853 & GAACAGAATGGAACAGATGAC \\
$\boldsymbol{c}$ - $\boldsymbol{M Y \boldsymbol { B }}$ & TRCN0000295917 & ACTATTCCTATTACCACATTT \\
\hline
\end{tabular}

Table 7. List of MISSION® pLKO.1 shRNA sequences used in this study. 


\section{- Inducible gene downregulation:}

The "Tet-On" pTRIPZ shRNA system was used in this study to knockdown Reptin expression in an inducible manner. Both the scrambled control and shReptin vectors were obtained from Dr Lu Zhao (PhD thesis, Zhao, 2013). The shRNA sequence in the pTRIPZ system used in this study was the same as the one in the pGIPZ system (Table 6). A schematic diagrams of the pTRIPZ(pgk) construct used in this study is shown in Figure 10. The pTRIPZ(pgk) is an inducible lentiviral expression vector, in which the reverse tetracycline transactivator 3 (rtTA3) gene is linked to the puromycin resistance gene by an internal ribosomal entry sequence (IRES) and driven off by the phosphoglycerate kinase (PGK) promoter. The constitutively expressed rtTA3 protein is only able to bind the tetracycline inducible promoter (TRE) in the presence of doxycycline, and to drive expression of the turbo Red Fluorescence Protein (tRFP) and shRNAs, embedded in the microRNA (miR) 30 sequence. 


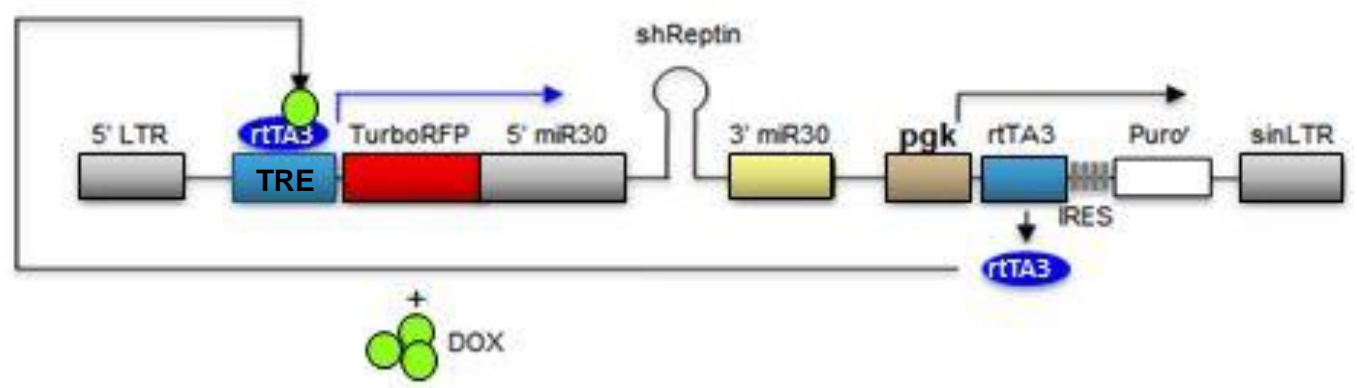

Figure 10. Diagram of the pTRIPZ (PGK) inducible system used in this study.

The diagram depicts the inducible system for Reptin depletion used in this study. Adapted from (PhD thesis, Zhao, 2013). LTR, long terminal repeat; $\psi$, viral packaging signal; RRE, Rev-Response Element; TRE, Tet-inducible promoter; shRNA, short hairpin RNA; PGK, murine phosphoglycerate kinase promoter; rtTA3, Reverse tetracycline-transactivator 3 for tetracycline-dependent induction of the TRE promoter; Puro ${ }^{\mathrm{r}}$, puromycin resistance gene; IRES, Internal ribosome entry site; WPRE, Woodchuck hepatitis posttranscriptional regulatory element enhances transgene expression in the target cells; AMPr, Ampicillin resistance gene for bacterial selection. 


\subsubsection{Western Blot analysis}

Cells were harvested and washed with PBS by centrifugation at $300 \mathrm{x} g$ for 5 minutes at $4^{\circ} \mathrm{C}$. Cell pellets were lysed using $60 \mu \mathrm{l}$ of $1 \mathrm{x}$ RIPA (Radio Immunoprecipitation Assay, Cell Signaling) per $10^{6}$ cells. The lysates were incubated on ice for 30 minutes, centrifuged at $16,000 \mathrm{xg}$ for 10 minutes at $4^{\circ} \mathrm{C}$ and pellets discarded. Protein concentration was measured using the Bradford reagent (Bio-Rad), by measuring the absorbance at a wavelength of $595 \mathrm{~nm}$ using the Infinite ${ }^{\circledR}$ F200 PRO NanoQuant (Tecan) absorbance reader. Then, $30 \mu \mathrm{g}$ to $60 \mu \mathrm{g}$ of protein were denatured at $95^{\circ} \mathrm{C}$ with Dithiothreiothreitol (DTT) sample buffer (200mM DTT, $2 \%$ w/v sodium dodecyl sulphate (SDS), $10 \%$ v/v Glycerol, 0.02\% w/v Bromophenol blue, $125 \mathrm{mM}$ Tris-HCL (tris[hydroxymethyl]aminomethane) [pH6.8]) for $5 \mathrm{~min}$ and were separated by electrophoresis in $10 \%$ SDS-PAGE gels. After that, samples were transferred to a polyninylidenefluoride (PVDF) membrane (Millipore). Membranes were then blocked in PBS with 5\% non-fat milk and 0.2\% v/v Tween-20 (SigmaAldrich), immunoblotted using specific primary antibodies (Table 8), detected using the appropriate secondary horseradish peroxide-conjugated antibodies and visualised using a chemiluminescence reagent (GE Healthcare). 


\begin{tabular}{|c|c|c|}
\hline ANTIBODY & SUPPLIER & DILUTION \\
\hline Reptin 52 (42) & Santa Cruz Biotechnology & $1: 1000$ \\
\hline Reptin (2E9-5) & Sigma Aldrich & $1: 1000$ \\
\hline Pontin 52 (N-15) & Santa Cruz Biotechnology & $1: 1000$ \\
\hline c-MYC (Y69) & Abcam & $1: 1000$ \\
\hline EPC1 & Santa Cruz Biotechnology & $1: 1000$ \\
\hline EPC2 & Abcam & $1: 1000$ \\
\hline MTBP (B-5) & Santa Cruz Biotechnology & $1: 500$ \\
\hline c-MYB (1-1) & Millipore & $1: 1000$ \\
\hline c-MYB (H-141) & Santa Cruz Biotechnology & $1: 1000$ \\
\hline c-MYB (EPR718) & Abcam & $1: 1000$ \\
\hline MIZ-1 & Cell Signaling Technology & $1: 500$ \\
\hline$\alpha$-Actin & Santa Cruz Biotechnology & $1: 2000$ \\
\hline GAPDH & Santa Cruz Biotechnology & $1: 1000$ \\
\hline Clathrin & Santa Cruz Biotechnology & $1: 1000$ \\
\hline Anti-HA & Roche & $1: 500$ \\
\hline
\end{tabular}

Table 8. List of primary antibodies used for western blot analysis in this study.

\subsubsection{RNA isolation, cDNA preparation and qRT-PCR}

Total RNA was isolated from the cells using the RNeasy Mini Kit (Qiagen) according to manufacturer's instructions. The cells were disrupted by adding lysis buffer RLT, containing 2-mercaptoethanol, followed by vortexing at the highest 
setting for 30 seconds to ensure homogenization of the samples. One volume of $70 \%$ ethanol was added to the samples and the mixture was transferred to an RNeasy spin column. After centrifuging the column for 15 seconds at $13,000 \mathrm{xg}$, the flow through was discarded. The column was washed with $700 \mu$ buffer RW1 first and then subsequently with $500 \mu \mathrm{l}$ wash buffer RPE. RNA was eluted with $30 \mu$ of RNase free water and the concentration determined using a spectrophotometer (NanoDrop ND-1000, Lebtech International).

RNA was converted into cDNA using the High Capacity RNA-to-cDNA Kit (Applied Biosystems) according to manufacturer's instructions. $1 \mu \mathrm{g}$ of RNA was converted using $1 \mu \mathrm{l} 20 \mathrm{X}$ Enzyme mix and $10 \mu \mathrm{l}$ of $2 \mathrm{X}$ RT Buffer Mix in a total volume of $20 \mu \mathrm{l}$. Quantitative RT-PCR (qRT-PCR) was performed on isolated mRNA using TaqMan probe based chemistry and the StepOnePlus ${ }^{\mathrm{TM}}$ Real-Time PCR system (Life Technologies). All primer/probe sets used were from Applied Biosystems, Life Technologies. Statistical analyses were performed and plots were constructed via GraphPad Prism (La Jolla, CA, USA).

\subsubsection{Co-immunoprecipitation (Co-IP) assays}

The Pierce ${ }^{\mathrm{TM}}$ Classic Magnetic IP/Co-IP Kit (Thermo scientific) was used to perform co-immunoprecipitation (Co-IP) assays, according to manufacturer's instructions. Briefly, 40x $10^{6}$ cells were lysed in $400 \mu 1$ lysis buffer. After cell lysis, $50 \mu 1$ of cell lysate were frozen to use as input and the remaining $350 \mu$ l were incubated with 10 $\mu \mathrm{g}$ (anti-Reptin) or $15 \mu \mathrm{g}$ (anti-c-MYB) antibody, or the equivalent amount of the 
immunoglobulin control (IgG) antibody. $50 \mu \mathrm{l}$ of magnetic beads were also added and the samples were incubated rotating overnight at $4^{\circ} \mathrm{C}$. The following day, the unbound fraction was frozen and the immune complexes were washed 5 times with IP Lysis/Wash Buffer and eluted in $50 \mu 1$ of elution buffer for 10 minutes. The eluates were then neutralised with $10 \mu \mathrm{l}$ of neutralisation buffer (Tris $\mathrm{pH}$ 8.5). Detection of the immune complexes in the whole cell lysate (input) and in the immunoprecipitated fraction was performed by western blot analysis.

To immunoprecipitate the HA-tagged Reptin, the Pierce ${ }^{\mathrm{TM}}$ Crosslink Magnetic IP/Co-IP Kit (Thermo scientific) was used, according to manufacturer's instructions. Briefly, $5 \mu \mathrm{g}$ of the anti-HA antibody were cross-linked to the beads for 30 minutes with DSS before incubating the lysates with the mixture of cross-linked beads over-night at $4{ }^{\circ} \mathrm{C}$. The rest of the protocol is as described above for the Classic Kit. Table 9 shows the list of antibodies used to immunoprecipitate the complexes. 


\begin{tabular}{cc}
\hline NAME & SUPPLIER \\
\hline Reptin 2E9-5 & Sigma Aldrich \\
\hline c-MYB (Y69) & Abcam \\
\hline Anti-HA & Roche \\
\hline c-MYC (Y69) & Abcam \\
\hline Normal Rabbit IgG (20-27) & Santa Cruz \\
\hline Normal Mouse IgG (12-371) & Millipore \\
\hline
\end{tabular}

Table 9. List of antibodies used in this study to perform immunoprecipitation assays.

\subsection{Cell Biology}

\subsubsection{Cell lines and tissue culture methods}

The 293FT (Invitrogen) packaging cell line was cultured in $10 \mathrm{~cm}$ culture dishes (NUNC) in Dulbecco's Modified Eagle's medium (DMEM, Sigma-Aldrich) supplemented with $10 \%$ heat-inactivated Foetal Calf Serum (FCS, Sigma-Aldrich), 100U/ml Penicillin (Sigma-Aldrich), $100 \mu \mathrm{g} / \mathrm{ml}$ Streptomycin (Sigma-Aldrich) and 2 mM L-glutamine (Sigma-Aldrich) (complete DMEM). Stock cells were also supplemented with $500 \mu \mathrm{g} / \mathrm{ml}$ Geneticin (G418 Sulfate, ThermoFisher Scientific), to select them, and re-plated every 3 to 4 days. To re-plate them, cells were washed with $5 \mathrm{~mL}$ PBS (Gibco) followed by trypsinization using $1 \mathrm{ml}$ of 1x Trypsin/EDTA (Gibco) for 5 minutes at $37^{\circ} \mathrm{C}$. Cells were then diluted in complete DMEM and centrifuged at $300 \mathrm{x} g$ for 5 minutes at RT, then seeded at a density of $0.35 \times 10^{6} / \mathrm{ml}$. 
The human leukaemic cell lines THP1 and U937 cells were cultured in Roswell Park Memorial Institute (RPMI) medium (Invitrogen), supplemented with 10\% FCS (Sigma-Aldrich), $100 \mathrm{U} / \mathrm{ml}$ Penicillin (Sigma-Aldrich), $100 \mu \mathrm{g} / \mathrm{ml}$ Streptomycin (Sigma-Aldrich) and $2 \mathrm{mM}$ L-glutamine (complete RPMI). Cell lines were cultured at a density of $0.5 \times 10^{6}$ cells $/ \mathrm{ml}$ and sub-cultured every three to four days. In some cases, cells were treated with $1 \mu \mathrm{g} / \mathrm{ml}$ Doxycycline (DOX) (ClontechTakara Bio) every 48 hours.

\subsubsection{Lentiviral packaging cell line transfection}

For lentiviral packaging cell line transfection, 293FT cells were seeded at a density of $0.75 \times 10^{6}$ per $10 \mathrm{~cm}$ petri dish three days before transfection, without Geneticin. For lentiviral transfection, $5 \mu \mathrm{g}$ of expression vector, $3.75 \mu \mathrm{g}$ of pCMV-PAX2 (which was kindly supplied by Professor D. Trono, Lausanne, Switzerland) and 1.5 $\mu \mathrm{g}$ of pVSV-G construct were incubated in $1 \mathrm{ml}$ Optimem (Gibco) plus $30 \mu \mathrm{l}$ polyethylenimine (PEI) (OriGene) reagent for 10 minutes at room temperature and then the PEI complexes were added to the cells. PEI containing medium was then replaced with $8 \mathrm{ml}$ of complete DMEM medium on the second day of transfection.

\subsubsection{Lentiviral transduction of target cells}

To transduce human leukaemic cell lines, 293FT retroviral supernatant was harvested 48 hours after transfection and cleared using Ministart $0.45 \mu \mathrm{m}$ filters (Sartorius Stedim Biotech). Supernatant was then diluted at a ratio of 1:1 with complete RPMI containing leukemic cells at a density of $1 \times 10^{6}$ cells $/ \mathrm{ml}$ and supplemented with 5 
$\mu \mathrm{g} / \mathrm{ml}$ of polybrene. Cells were then plated in a 24 well plate (1ml per well) and transduced by spinoculation at $700 \mathrm{xg}$ for 45 minutes at $25^{\circ} \mathrm{C} .24$ hours following transduction, $1 \mathrm{ml}$ of complete RPMI was added to the cells. 48 hours following transduction, cells were harvested and plated in complete RPMI and selected with 2 $\mu \mathrm{g} / \mathrm{ml}$ puromycin for 3 days.

The inducible pTRIPZ RFP-shReptin and the Reptin Dominant Negative (D299N) lentiviral vectors used in this study were generated by Lu Zhao ( $\mathrm{PhD}$ thesis, Zhao, 2013). To generate the CSGWp-3xHa-Reptin vector, the 3xHA-Reptin fragment was obtained by PCR from the pCDNA-3xHA-Reptin plasmid from Addgene (www.addgene.com, plasmid \#51636) using the following primers:

F: 5'- ATACGACTCACTATAGGGAGACCCAAGC - 3'

R: 5' - TCAGGAGGTGTCCATGTGCGCATGACTG - 3'

50 ng of the PCR fragment were then ligated into the pGEM®-T Easy vector and transformed into DH5aTM chemically competent cells. The isolated DNA was digested with BamHI and MluI restriction enzymes (Thermo Scientific) and cloned into the pCSGW vector previously digested with BamHI and MluI restriction enzymes (Thermo Scientific). The ligation products were transformed into Stbl3 chemically competent cells. 


\subsubsection{Flow Cytometry}

To detect expression of the Red Fluorescent Protein, cells were washed with $1 \mathrm{ml}$ of wash buffer (PBS supplemented with $0.05 \%$ w/v sodium azide) and re-suspended in $300 \mu \mathrm{L}$ of wash buffer. Apoptosis was detected using the Annexing V Apoptosis Detection Kit I (BD Biosciences). Briefly, cells were washed with $1 \mathrm{ml}$ of $1 \mathrm{x}$ Annexin $\mathrm{V}$ binding buffer from a 10x stock solution (0.1M Hepes [pH 7.4], 1.4M $\mathrm{NaCl}, 25 \mathrm{mM} \mathrm{CaCl} 2$ solution) and stained with $5 \mu \mathrm{l}$ of Annexin $\mathrm{V}^{\mathrm{APC}}$ in the dark for 15 minutes at room temperature. Cells were then washed and re-suspended in $300 \mu \mathrm{L}$ of $1 \mathrm{x}$ binding buffer. $10 \mu \mathrm{L}$ of 4', 6-diamidino-2-phenylindole (DAPI) were added to detect apoptotic cells. Cells were analysed on an LSRII analyser (BD Biosciences) and the data was analysed using Summit 4.3 software (Dakocytomation).

\subsubsection{Dead cell removal}

In some cases, apoptosis analysis of transduced cells was performed after removal of dead cells with the Dead Cell Removal kit (Miltenyi Biotec), to obtain equivalent numbers of viable cells. Cells were centrifuged at $300 \mathrm{x} g$ for 5 minutes, resuspended in $100 \mu \mathrm{l}$ of Dead Cell Removal MicroBeads and incubated at room temperature for 15 minutes. MS columns (Myltenyi Biotech) were prepared by being washed with $1 \mathrm{x}$ binding buffer, and were placed into a magnetic field of a MACS® separator. $500 \mu 1$ of $1 \mathrm{x}$ binding buffer was added to the cells and the mixture was loaded into the column. The effluent containing the live cell fraction was collected and the column was washed 4 times with $500 \mu 1$ of $1 \mathrm{x}$ binding buffer to collect remaining live cells. Live cells were then centrifuged at $300 \mathrm{xg}$ for 5 minutes and resuspended in suitable growth medium. 


\subsubsection{Luciferase assay}

Luciferase signal from cells was measured using the Luciferase Assay system (Promega) according to manufacturer's instructions. Briefly, $1 \times 10^{3}$ cells were plated in a white 96-multiwell plate (Non-TC treated, Greiner Bio One) and washed with PBS. Following centrifugation, cells were re-suspended in $20 \mu \mathrm{l}$ x Lysis reagent and incubated on a shaker in the dark for 15 minutes. Then, $100 \mu l$ of Luciferase assay reagent was injected into each well by the injector in the Infinite ${ }^{\circledR} 200$ PRO plate reader (Tecan) and the level of luciferase signal in each well was measured.

\subsection{RNA-sequencing}

\subsubsection{RNA-seq Library preparation and Sequencing}

Total cellular RNA was extracted using the RNeasy Plus Mini Kit (Qiagen) according to manufacturer's instructions. $100 \mathrm{ng}$ of RNA per sample were analysed using Bioanalyzer 2100 (Agilent Technologies, Santa Clara, CA) to verify RNA integrity prior to amplification. The samples were submitted to UCL Genomics for RNA-seq and processed using Illumina's TruSeq RNA sample prep kit Version2 (p/n RS-122-2001) according to manufacturer's instructions (Illumina, Cambridge, UK). Briefly, mRNA was selected using paramagnetic dT beads and fragmented by metal hydrolysis to approximately 150 bp lengths. Random primed cDNA was then generated and adapters compatible with Illumina sequencing were ligated before being enriched by 12 cycles of PCR. Libraries were quantified, normalised and pooled before sequencing on an Illumina NextSeq 500, generating approximately 20 million 43 bp read pairs per sample. The RNA express workflow on Illumina 
BaseSpace was used to determine differential gene expression in 5 or 3 biological replicates of Reptin shRNA transduced samples treated with or without DOX. In brief, alignment of RNA-seq reads to the human UCSC mm10 genome and mapping to genes was performed with STAR aligner (Dobin et al., 2013). Differential gene expression between the two biological replicates each was calculated with DESeq2 (Anders and Huber, 2010).

\subsubsection{Gene set enrichment analysis (GSEA)}

A list of differentially expressed genes was obtained from the RNA express workflow on Illumina BaseSpace ${ }^{\circledR}$ (basespace.illumina.com). The lists of genes were ordered according to differential expression between control cells and Reptin depleted cells and the "Low" expressed genes were removed from the output file. The GSEAPreranked tool of GSEA (http://www.broad.mit.edu/gsea/) was used to examine the distribution of the c-MYC, c-MYB and LSC signatures. The c-MYC signatures were derived from Schuhmscher et al. (Schuhmacher et al., 2001) and Kim et al. (Kim et al., 2006b). The c-MYB signatures were derived from Zhao et al. (Zhao et al., 2011). These were genes bound by c-MYB in mouse myeloid ERMYB cells and deregulated in THP1 cells following siRNA mediated c-MYB silencing (Suzuki et al., 2009) either decreasing in expression (MYB activated) or increasing in expression more than 1.5-fold (MYB repressed). LSC signatures were derived from Somervaille et al. (Somervaille et al., 2009), consisting of probe sets positively correlated with LSC frequency (Positive LSC signature) and negatively correlated with LSC frequency (Negative LSC signature). Mouse gene names were converted 
into human gene names with HCOP using the HGNC orthologs. A negative enrichment score indicates anti-correlation.

\subsubsection{PANTHER analysis}

Gene ontology and protein class analysis of the differentially expressed proteins were performed using PANTHER classification system (version 9.0) available on http://pantherdb.org.

\subsection{Chromatin immunoprecipitation (ChIP) assays}

$25 \times 10^{6}$ THP-1 cells transduced with a pLKO.1 scrambled control shRNA or the pLKO.1 shRNA targeting Reptin (clone ID: TRCN0000051563) were cross-linked with $2 \mathrm{mM}$ disuccinimidyl glutarate (DSG) for 30 minutes at room temperature on a rotating wheel. After washing the samples 3 times with PBS, cells were fixed with $1 \%$ formaldehyde for $15 \mathrm{~min}$ at room temperature on a rotating wheel. The reaction was stopped by adding glycine to final concentration $0.25 \mathrm{M}$ for 5 minutes at room temperature. Fixed cells were rinsed twice with $20 \mathrm{ml}$ of cold PBS and resuspended in $50 \mathrm{ml}$ of lysis buffer A $(0.25 \%$ TritonX100, $10 \mathrm{mM}$ EDTA, $0.5 \mathrm{mM}$ EGTA, 20 $\mathrm{mM}$ HEPES [pH 7.6]) and incubated for $10 \mathrm{~min}$ at $4{ }^{\circ} \mathrm{C}$ in a rotating wheel. Cells were centrifuged for 5 minutes at $1350 \mathrm{xg}$, resuspended in $50 \mathrm{ml}$ lysis buffer B (150 $\mathrm{mM} \mathrm{NaCl}, 10 \mathrm{mM}$ EDTA, $0.5 \mathrm{mM}$ EGTA, $20 \mathrm{mM}$ HEPES [pH 7.6]) and incubated for $10 \mathrm{~min}$ at $4^{\circ} \mathrm{C}$ in a rotating wheel. Cells were then pelleted for 5 minutes at 1350 $\mathrm{x} g$, resuspended in $1.5 \mathrm{ml}$ of incubation buffer (0.15\% SDS, $1 \%$ TritonX100, $150 \mathrm{nM}$ 
$\mathrm{NaCl}, 10 \mathrm{mM}$ EDTA, $0.5 \mathrm{mM}$ EGTA, $20 \mathrm{mM}$ HEPES [pH 7.6]) plus protease inhibitors (Sigma Aldrich) and incubated for 30 minutes at $4{ }^{\circ} \mathrm{C}$ on ice. Lysates were then sonicated for 6 cycles (30 sec on / $30 \mathrm{sec}$ off) in the Bioruptor ${ }^{\circledR}$ Pico water bath-sonicator (Diagenode) and centrifuged at 16,000 $\mathrm{xg}$ for $10 \mathrm{~min}$. The cleared supernatant was stored at $-80^{\circ}$ C. $500 \mu \mathrm{l}$ of sonicated chromatin were diluted 10 times in ChIP Dilution Buffer (SDS 0.01\%, Triton X-100 1.1\%, 1.2 mM EDTA [pH 8], $16.7 \mathrm{mM}$ Tris- $\mathrm{HCl} \mathrm{pH} 8$ and $167 \mathrm{mM} \mathrm{NaCl}$ ) and were incubated rotating at $4^{\circ} \mathrm{C}$ overnight, with $40 \mu \mathrm{l}$ beads (Protein A and G, Millipore) and 2-10 $\mu \mathrm{g}$ of specific antibody (Table 10). The beads were washed for 10 minutes once in $10 \mathrm{ml}$ of Low Salt Buffer (0.1\% SDS, 1\% TritonX100, 2 mM EDTA pH 8, 20 mM Tris-HCl [pH 8.1], $150 \mathrm{mM} \mathrm{NaCl}), 10 \mathrm{ml}$ of High Salt Buffer (0.1\% SDS, $1 \%$ TritonX100, $2 \mathrm{mM}$ EDTA, $20 \mathrm{mM}$ Tris- $\mathrm{HCl}$ [pH 8.1], $500 \mathrm{mM} \mathrm{NaCl}), 10 \mathrm{ml}$ of $\mathrm{LiCl}$ Buffer $(0.25 \mathrm{M}$ LiCl, 1\% NP-40, 1 mM EDTA, $10 \mathrm{mM}$ Tris-HCl $\mathrm{pH}$ 8.1) and in TE buffer (10 mM Tris-HCl pH 8.1, $1 \mathrm{mM}$ EDTA). ChIPed material was eluted by two 1-hour incubations at $55^{\circ} \mathrm{C}$ with $200 \mu$ Elution Buffer (1\% SDS, $100 \mathrm{mM}$ NaHCO3). Chromatin was reverse-crosslinked by adding $16 \mu \mathrm{l}$ of $\mathrm{NaCl} 5 \mathrm{M}$ and incubated at $65^{\circ} \mathrm{C}$ overnight. DNA was subjected to RNase and proteinase $\mathrm{K}$ digestion and extracted by using the MinElute PCR purification kit (Qiagen). The DNA was then subjected to quantitative PCR (qPCR) analysis using the sensiFAST SYBR Hi-ROX Kit (Bioline), according to manufacturer's instructions. Briefly, a master mix containing 1x SensiFAST SYBR® Hi-ROX mix, $100 \mathrm{nM}$ or $400 \mathrm{nM}$ primers and nuclease-free water was mixed with $2 \mu 1$ of eluted DNA per well in a 96 well plate. A qPCR was performed in triplicate for each DNA sample using the StepOnePlus ${ }^{\mathrm{TM}}$ Real-Time PCR system (Life Technologies). Table 11 shows the list of primers and 
concentrations used for ChIP qPCR analysis. Table 12 shows a list of primers that were tested during the optimisation process but finally not used. To determine the efficiency and the limit of detection of the primers used for ChIP experiments, a standard curve from a serial dilution of input DNA was performed using different primer concentrations (Figure 11). Melt curve analysis was used to validate that the qPCR primers had produced a single specific product/amplicon, by detecting a single peak (Figure 12).

\begin{tabular}{ccc}
\hline NAME & VOLUME & SUPPLIER \\
\hline c-MYB (D-7) & $5 \mu \mathrm{l}(5 \mu \mathrm{g})$ & Santa Cruz \\
c-MYB (1-1) & $25 \mu \mathrm{l}(5 \mu \mathrm{g})$ & Biotechnology \\
H3K27ac (chip grade) & $2 \mu \mathrm{l}(2 \mu \mathrm{g})$ & Millipore \\
\hline
\end{tabular}

Table 10. List of antibodies used for ChIP assays in this study. 


\begin{tabular}{ccc}
\hline GENE & $\begin{array}{c}\text { SEQUENCE } \\
\text { (Reference) }\end{array}$ & CONCENTRATION \\
NAME & AACTGATCACTAACCACAACCAGTTC & $400 \mathrm{nM}$ \\
MPO F & $($ Zhao et al., 2014) & \\
MPO R & GAGACCGTTGGGCTTCACA & $400 \mathrm{nM}$ \\
GAPDH F & TCGACAGTCAGCCGCATCT & $400 \mathrm{nM}$ \\
GAPDH R & CTAGCCTCCCGGGTTTCTCT & $400 \mathrm{nM}$ \\
GD21 & GGGGGATCAGATGACAGTAAA & $100 \mathrm{nM}$ \\
GD21 & AATGCCAGCATGGGAAATA & $100 \mathrm{nM}$ \\
\hline
\end{tabular}

Table 11. List of primer sequences used for ChIP assays.

Table showing the primer sequences and concentrations used for ChIP assays in this study. 


\begin{tabular}{|c|c|}
\hline GENE/PRIMER NAME & SEQUENCE (Reference) \\
\hline CDK6 Forward 1 & AGGCCAAAGACATAAAGTGC \\
\hline CDK6 Reverse 1 & AAGCAGAACAGTCGGATAGT \\
\hline CDK6 Forward 2 & GGGTTAGCAAATGCCTCTAAT \\
\hline CDK6 Reverse 2 & ATGTGATAGCTGCTTGTACC \\
\hline c-MYC Forward 1 & AAATACCCATCACCTTCTGC \\
\hline c-MYC Reverse 1 & GGTTCTCCATAGGGTGATGT \\
\hline c-MYC Forward 2 & TTCCAATCTGACTTGTGTCC \\
\hline c-MYC Reverse 2 & TAGCTTCTGCCCTTTTCTTT \\
\hline c-MYC Forward 3 & $\begin{array}{l}\text { AAAAGGGGAAAGAGGACCTGG } \\
\text { (Zhao et al., 2012) }\end{array}$ \\
\hline c-MYC Reverse 3 & $\begin{array}{c}\text { CCTAAAAGGGGCAAGTGGAGAG } \\
\text { (Zhao et al., 2012) }\end{array}$ \\
\hline SAMSN1 Forward 1 & TGCTTTCCACAACAGTACTAAA \\
\hline SAMSN1 Reverse 1 & ATGAAGACAATGTCACTTTTGC \\
\hline SAMSN1 Forward 2 & ATCTTTTCGGGTTCATGTTTTG \\
\hline SAMSN1 Reverse 2 & GTGCAGGGCATTAAAAGTAAAA \\
\hline PLD6 Forward 1 & CACAACTGTCACGCACCAAG \\
\hline PLD6 Reverse 1 & GTGGTCACCTGTTGAGGGTT \\
\hline PLD6 Forward 2 & AGTCTCTTCCTCTGTTTATGCT \\
\hline PLD6 Reverse 2 & САCСТTCACTGTCTTCTCG \\
\hline MYADM Forward 1 & GCTGTTGTGTCTTCCTGTTTTGT \\
\hline MYADM Reverse 1 & CGCCCCCCAGAGTAAAAGT \\
\hline MYADM Forward 2 & GTTGTGTCTTCCTGTTTTGTC \\
\hline
\end{tabular}




\begin{tabular}{cc}
\hline MYADM Reverse 2 & CCCAGAGTAAAAGTAGCGT \\
GD18 Forward & ACTCCCCTTTCATGCTTCTG \\
GD18 Reverse & AGGTCCCAGGACATATCCATT \\
HUMAN NEGATIVE & Active Motif (Catalog No. 71001) \\
CONTROL Primer Set 1 & \\
HUMAN NEGATIVE & Active Motif (Catalog No. 71002) \\
CONTROL Primer Set 2 & \\
\hline
\end{tabular}

Table 12. List of primer sequences tested for c-MYB ChIP assays.

Table showing a list of primers tested for ChIP assays but not used in this study. 


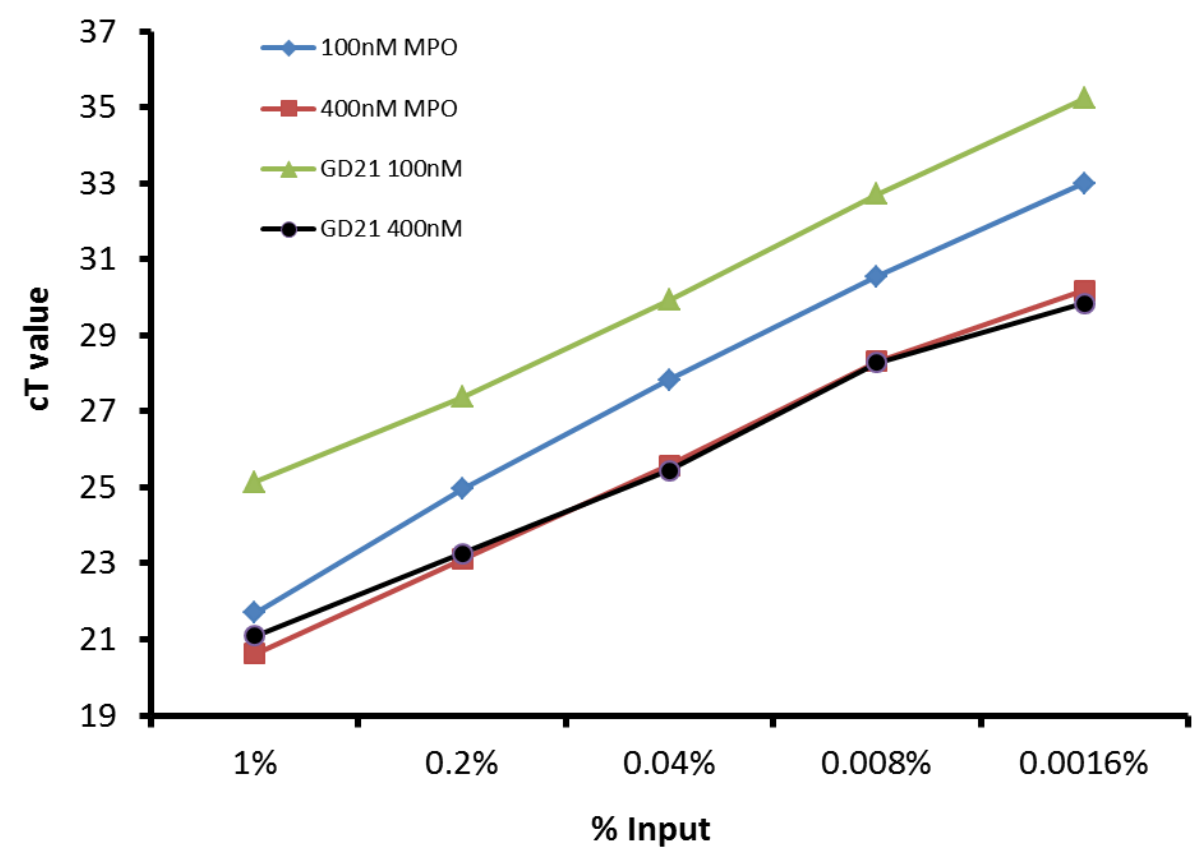

Figure 11. Standard curves of the primers used for ChIP assays.

Standard curves of MPO and GD21 specific SYBR Green qPCR primers at different concentrations (100 $\mathrm{nM}$ or $400 \mathrm{nM}$ ). Data were generated using 5-fold serial dilutions of known concentrations of chromatin input DNA. Data represent a single experiment. 

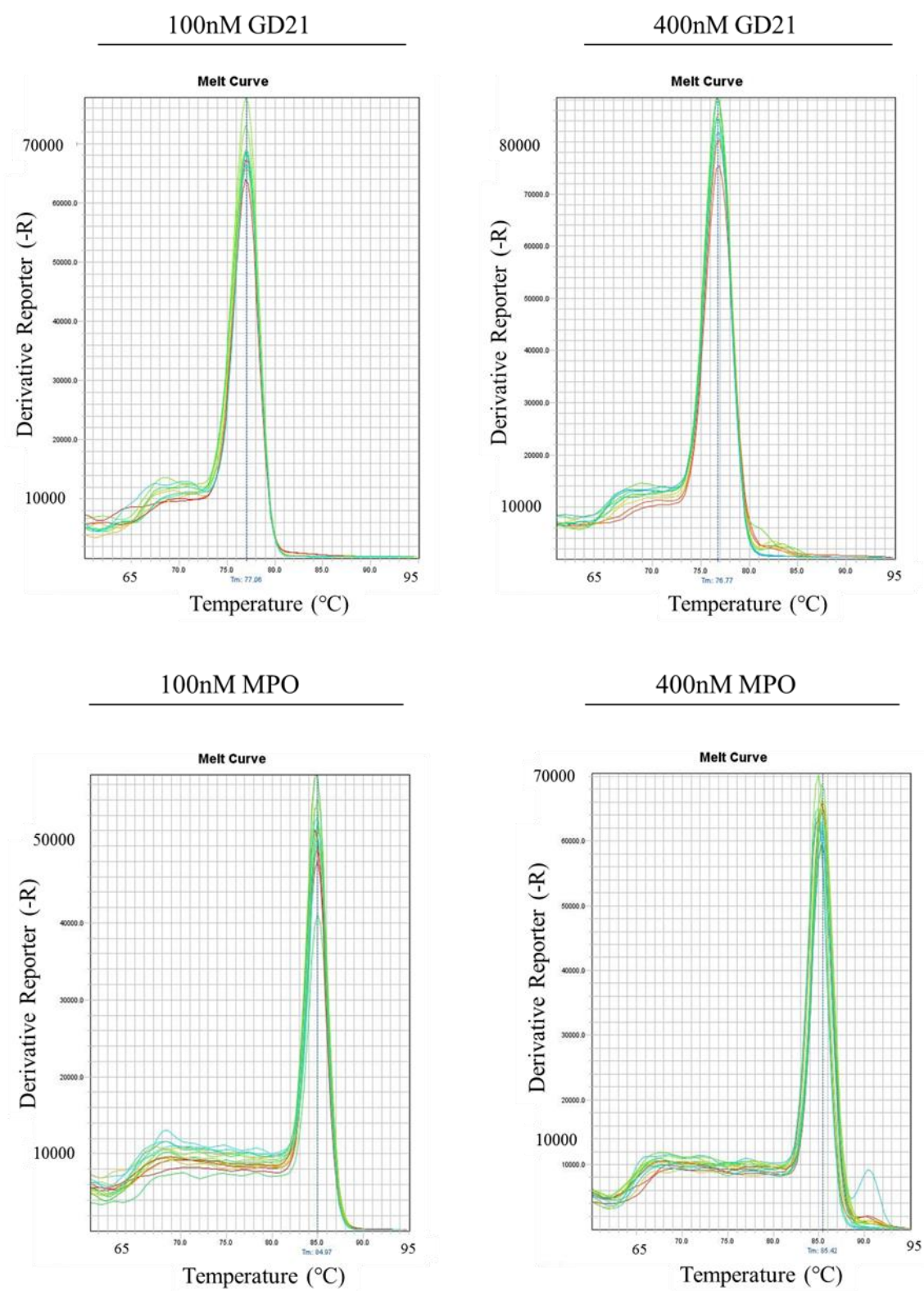

\section{Figure 12. Melt curves from qPCR of GD21 and MPO.}

An amplicon from the promoter region of the MPO gene, or a gene desert region in chromosome 21 (GD21), reveals a single peak following melt curve analysis using input DNA in a 5-fold serial dilution. Primers were tested at different concentrations as indicated (100 nM or $400 \mathrm{nM})$. Data shown here represent a single experiment, but melt curves were generated for every ChIP qPCR experiment. 


\subsection{Animal work}

All mice were maintained in the animal facilities of the UCL Institute of Child Health and experiments were performed according to United Kingdom Home Office regulations. Dr. Owen Williams and Dr. Luca Gasparoli performed xenotransplantations in this study on 5-10 week old NOD.Cg-Prkdc ${ }^{\text {scid }} \mathrm{Il} 2 \mathrm{rg}^{\mathrm{tm} 1 \mathrm{Wjl}} / \mathrm{SzJ}$ (NSG) mice. These mice lack mature T cells, B cells, and natural killer (NK) cells and are deficient in multiple cytokine signalling pathways. Furthermore, they have many defects in innate immunity. NSG mice have features of the NOD/ShiltJ background, the combined immune deficiency mutation (scid) and IL-2 receptor $\gamma$ chain deficiency. They have been shown to support high levels of human haematopoietic stem cell engraftment (McDermott et al., 2010).

The NSG mice used in this study were recipients of THP-1 clones generated in a previous study (PhD study, Zhao, 2013) expressing an inducible Reptin-specific shRNA (shRep) or the control scrambled shRNA (shSCR). In the present study, these cells were transduced with a lentiviral vector containing a luciferase (LUC2)IRES-EGFP cassette. Mice were injected intravenously in the lateral tail vein with $0.5 \times 10^{6}$ transduced cells per mouse. Longitudinal analysis of leukaemia progression was performed using an IVIS Lumina Series III pre-clinical in vivo imaging system (Perkin Elmer). D-luciferin $(10 \mu \mathrm{l}$ per g body weight of $15 \mathrm{mg} / \mathrm{ml}$ stock in PBS) was administered via intraperitoneal injection and mice imaged 5 minutes later, after anaesthetisation. Mice were sacrificed when they developed clinical signs of disease. 


\section{CHAPTER III - Investigating the consequences in vivo of conditional Reptin loss on human AML}

\subsection{Introduction}

In a previous study from our laboratory, the loss of the MLL-fusion in conditionally immortalised mouse haematopoietic progenitor cells led to a decrease in the mRNA levels of Reptin (PhD thesis, Walf-Vorderwülbecke, 2009). Reptin, which encodes an ATPase with several cellular functions, was found to be a downstream target gene of the MLL-AF9 fusion via c-MYB induction, as Reptin transcript decreased upon cMYB depletion in MLL-AF9 expressing cells (Osaki et al., 2013). Further studies in our laboratory showed that Reptin is essential for the oncogenic activity of MLLfusions in human AML, as short hairpin RNA (shRNA)-mediated depletion of Reptin resulted in a striking decrease in proliferation, an increase in apoptosis and a decrease in clonogenic potential of human AML cells (Osaki et al., 2013).

In order to determine whether Reptin is a potential target for novel therapies in established leukaemia, a previous study in our group used mouse transplantations to examine the effect of Reptin depletion in vivo ( $\mathrm{PhD}$ thesis, Zhao, 2013). Reptin loss eliminated established mouse MLL-induced leukaemias. Moreover, a dominant negative mutant of Reptin, devoid of ATPase activity, also impaired leukaemia progression in vivo in mouse transplantations ( $\mathrm{PhD}$ thesis, Zhao, 2013). In this study we examined the effect of inhibiting Reptin expression in established human AML 
using leukaemic xenotransplantations in mice. For this purpose, we used conditional shRNA clones of the MLL-AF9 expressing human AML cell line THP-1, generated by a previous member of the group (PhD thesis, Zhao, 2013). The clones were generated from single cell sorted THP-1 cells expressing an inducible Reptin-specific shRNA previously found to efficiently knockdown Reptin expression, or the control scrambled shRNA (shSCR). Using a clonal population of cells allowed us to achieve uniform induction of shRNA expression, particularly important when evaluating the consequences of Reptin knockdown in vivo in established leukaemia. 


\subsection{Results}

\subsubsection{Validating Reptin knockdown in inducible THP-1 clones}

Prior to initiating in vivo experiments, we first decided to validate the levels of Reptin knockdown in the previously mentioned inducible THP-1 clones. In the inducible "Tet-On" pTRIPZ lentiviral expression system, addition of doxycycline (DOX) to the culture medium allows binding of the constitutively expressed rtTA3 protein to the tetracycline inducible promoter element (TRE), to drive expression of the turbo Red Fluorescence Protein (tRFP) and the shRNAs, embedded in the microRNA (miR) 30 sequence (Figure 10). Treatment with DOX of the inducible clones expressing the shRNA against Reptin (shRep R1 and R6) resulted in a marked decrease of Reptin protein in comparison to the DOX treated clones expressing a control scrambled shRNA (shSCR C2 and C4) (Figure 13), confirming results from a previous study (PhD thesis, Zhao, 2013). Our western blot results also showed that clones R1 and R6 expressed lower levels of Reptin protein than control clones C2 and C4, even in the absence of DOX treatment (Figure 13), suggesting some degree of 'leakiness' in the 'Tet-On' system. 


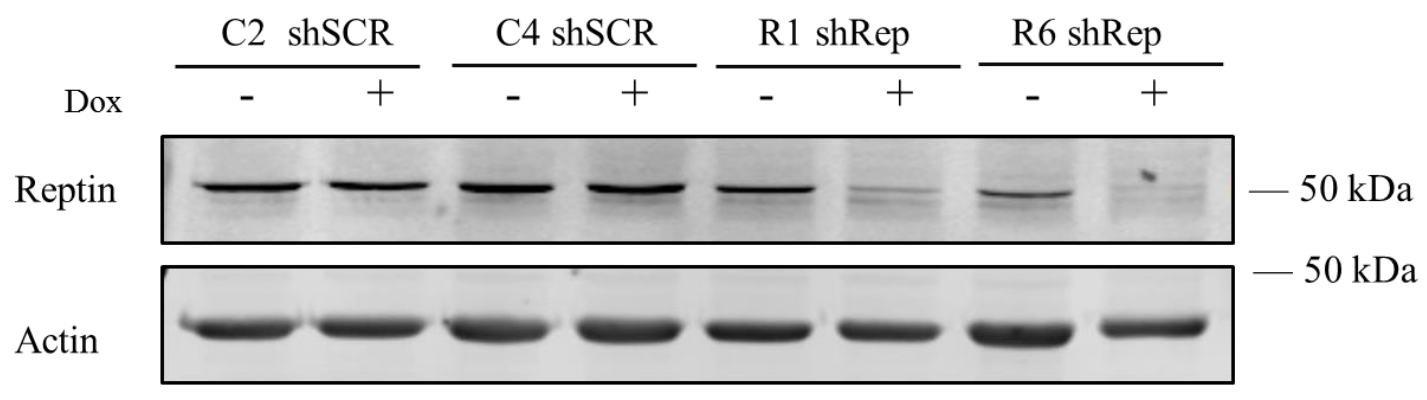

Figure 13. Validation of Reptin depletion in THP-1 inducible clones.

Western blot analysis of Reptin protein expression in C2 and C4 scrambled shRNA (shSCR), and R1 and R6 Reptin shRNA (shRep) inducible THP-1 clones, following continued treatment with or without DOX for 7 days. Actin was used as a loading control. This is a representative image of two independent experiments. 


\subsubsection{Characterising luciferase transduced inducible THP-1 clones}

After confirming efficient depletion of Reptin, we transduced an inducible Scrambled control clone (shSCR C4) and an inducible shRNA targeting Reptin expressing clone (shRep R6) with a lentiviral vector containing the firefly luciferase gene. The luciferase gene encodes an enzyme that catalyses the oxidation of D-luciferin, resulting in the emission of light. Thus, injection of mice with D-luciferin has been used to detect luciferase expressing cells in vivo and to follow the progression of leukaemia in xenografted recipient mice. This non-invasive bioluminescence imaging technique in mice had already been validated in our laboratory with control THP-1 cells.

A small proportion of the luciferase transduced clones were then subjected to a luciferase assay to confirm luciferase expression in both C4 shSCR and R6 shRep clones (Figure 14 A). Some cells were also induced with DOX for 3 days prior to subjecting them to a western blot assay to confirm that efficient depletion of Reptin protein was still possible after transduction with the luciferase expressing vector (Figure $14 \mathrm{~B}$ and C). 
A)

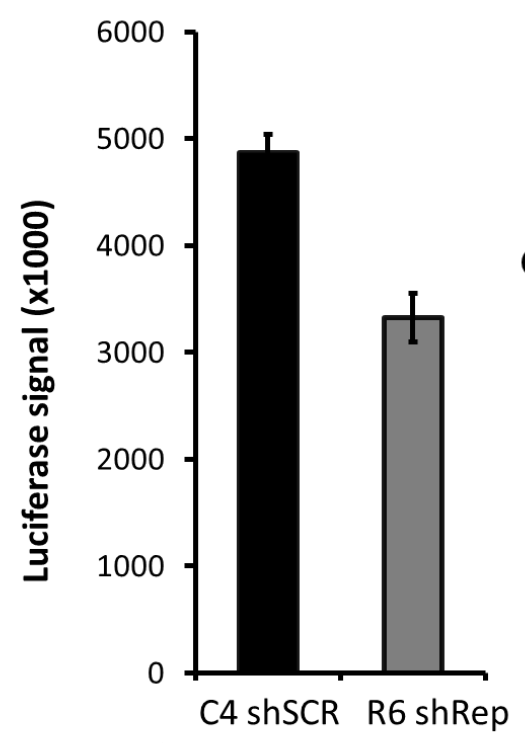

B)

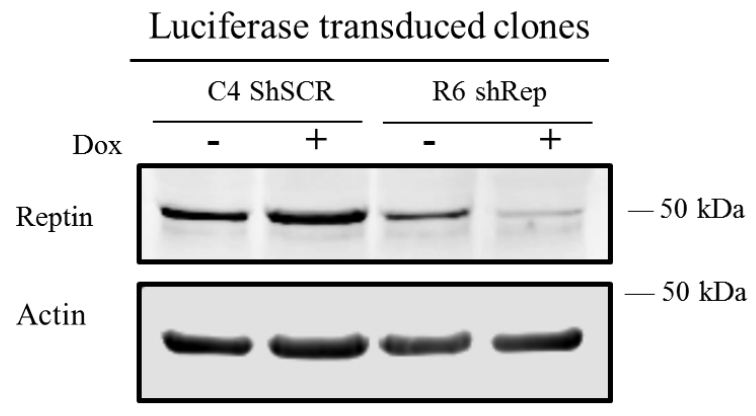

C)

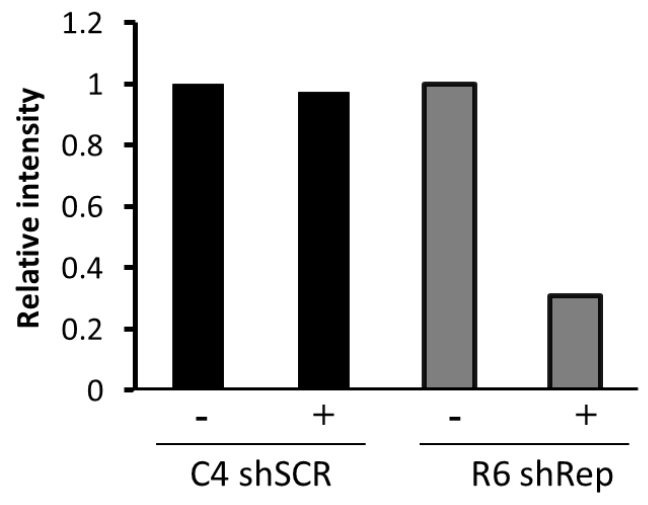

Figure 14. Characterisation of luciferase transduced inducible clones.

(A) The bar chart shows the luciferase signal of the luciferase transduced inducible scrambled shRNA (C4) and Reptin shRNA (R6) THP-1 clones after performing a luciferase assay. Luciferase signal was measured using Promega Luciferase assay kit. Columns represent means of triplicate measurements and error bars the SD. Western blot analysis of Reptin in luciferase transduced inducible THP-1 clones, following continued treatment with or without DOX for 3 days. The data was obtained from a single experiment performed on the cells that were transplanted to the NSG recipient mice. Actin was used as a loading control. (C) The bar chart shows the densitometric quantification of Reptin protein levels from (B) relative to Actin. 


\subsubsection{Reptin depletion eliminates established leukaemia in vivo}

To examine the effect of targeting Reptin in vivo, we transplanted NOD-SCID- $\gamma^{-/-}$ (NSG) mice with equal numbers of the luciferase-transduced inducible THP-1 clones (C4 and R6). 10 days after transplantation, we measured bioluminescence signal in all groups of mice to make sure all animals had similar levels of leukaemic burden before the start of DOX treatment (Figure 15). Immediately after, some recipient mice were subjected to DOX treatment by 3 rounds of daily intraperitoneal injection, followed by continual treatment with DOX in the food. Luciferase signal was measured every 7 or 14 days to monitor leukaemia progression, and DOX treatment was stopped in surviving mice after control mice had to be sacrificed. Remarkably, while induction of the scrambled shRNA did not delay leukaemic progression, suppression of Reptin delayed leukaemia progression in all of the DOX treated mice and resulted in complete remission in 5 out of 6 mice $(83.3 \%)$, even after termination of DOX treatment (Figure 16). 


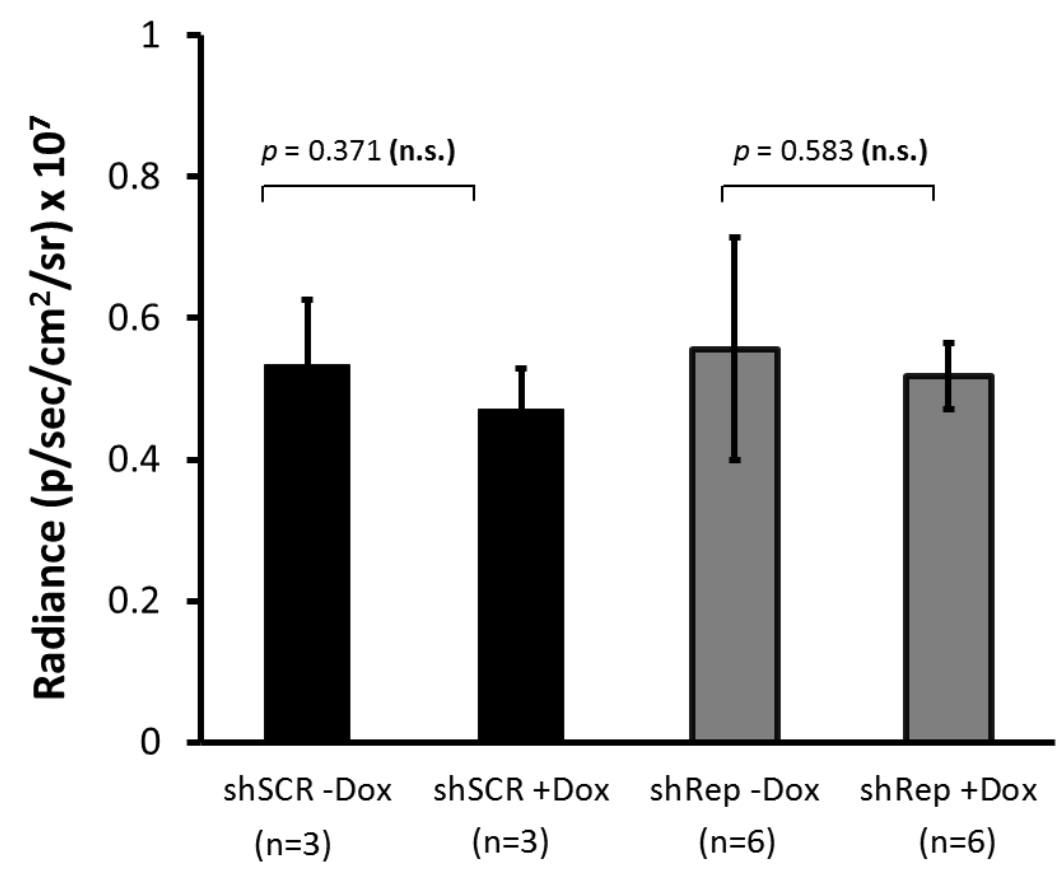

Figure 15. Bioluminescence levels in all groups of mice at the start of the experiment.

Bioluminescence signal was measured in NSG mice 10 days after transplantation using the IVIS Lumina Series III. Each bar represents the average signal for every experimental group of mice. Number of mice is indicated in brackets. Error bars represent SD. $P$-values were calculated using Student's unpaired $t$-test; (n.s.) not significant $(p \geq 0.05)$. 
A)

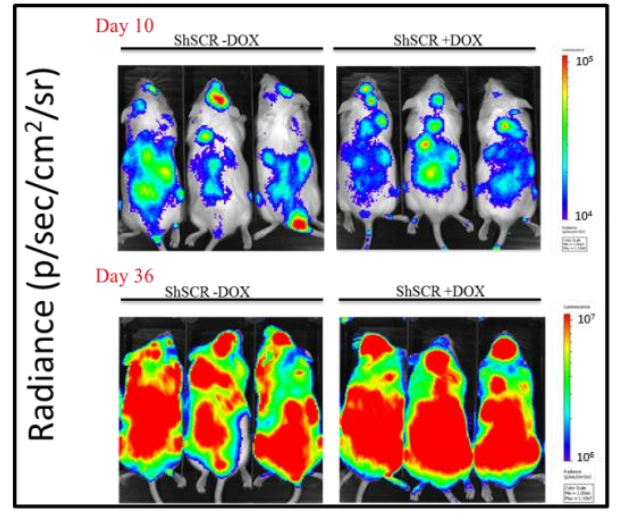

B)

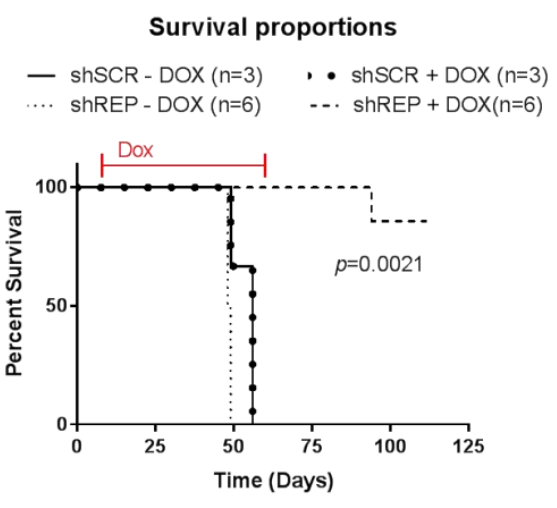

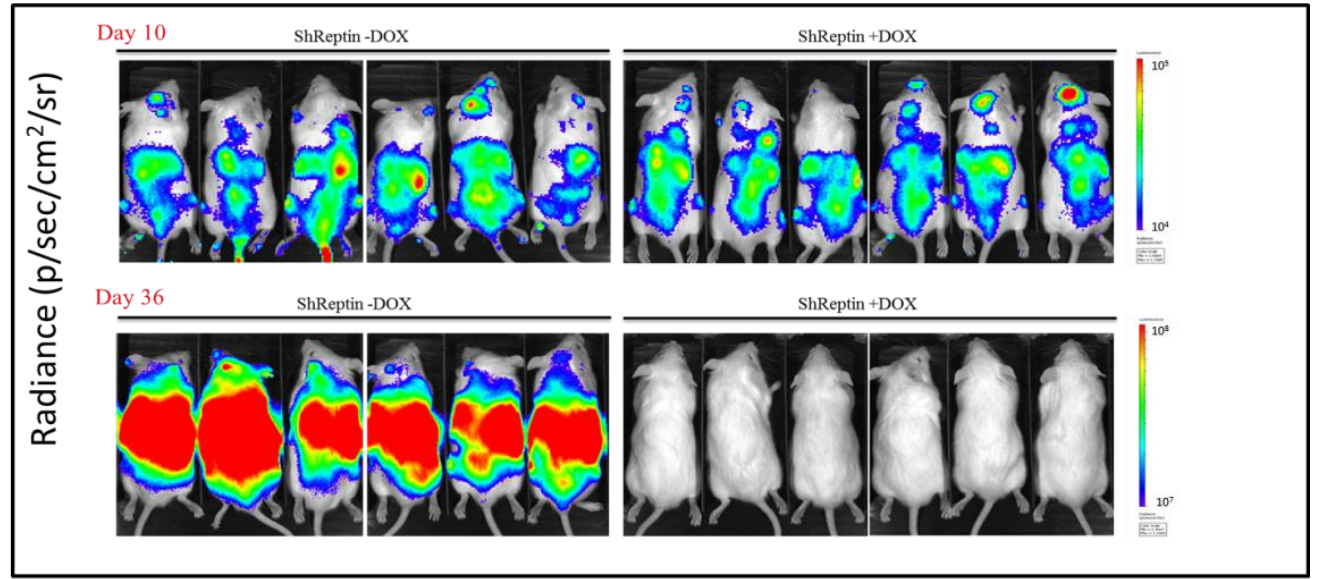

C)

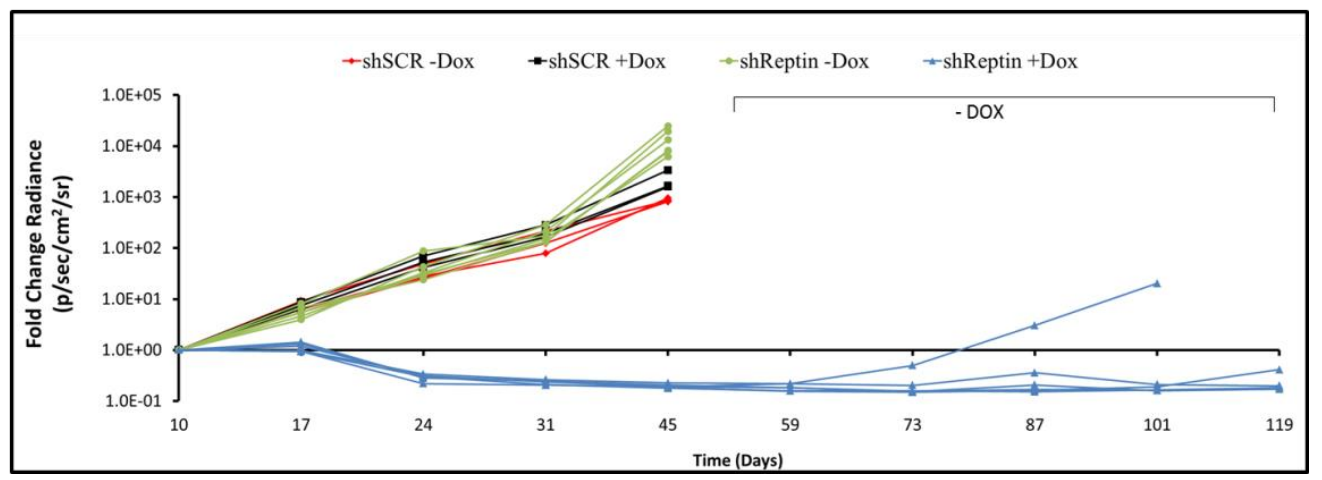

Figure 16. Reptin depletion delays or abolishes leukaemia progression in vivo.

(A) Bioluminescence images of NSG mice 10 days or 36 days after transplantation with $0.5 \times 10^{6}$ cells of luciferase-transduced inducible C4 (shSCR) or R6 (shRep) clones. 10 days after transplantation, some mice were subjected to DOX treatment (+Dox) and some were left untreated (-Dox). (B) The resulting Kaplan-Meier 
survival curve from (A). A $p$-value calculated using Mantel-Cox test is indicated in the plot. The red line on the curve indicates the duration of DOX treatment in + Dox treated mice. Group numbers are indicated in brackets. Mice were sacrificed upon developing signs of illness. (C) Line chart showing the luminescence radiance for each mouse from (A) measured at 7-day intervals starting from day 10 after transplantation. 


\subsection{Discussion}

Our laboratory has previously shown that Reptin is an essential downstream target gene of the MLL-fusions in AML (PhD thesis, Walf-Vorderwülbecke, 2009). Further research in our laboratory showed that Reptin is required for the proliferation and survival of $M L L$-rearranged and non- $M L L$-rearranged human AML cell lines (Osaki et al., 2013). Moreover, in a different study, the Reptin-related protein Pontin was identified as a critical mediator of AML1-ETO-induced leukaemia (Breig et al., 2014). Additionally, Reptin has also been found to be overexpressed and essential in in other types of cancer, including solid tumours such as breast cancer (Maslon et al., 2010) and hepatocellular carcinoma (Rousseau et al., 2007) (Haurie et al., 2009).

Previous work from our laboratory aimed to evaluate the efficacy of targeting Reptin in vivo in established leukaemia and this was done in two different ways: by using inducible clones expressing an shRNA to deplete Reptin, generated from mouse MLL-ENL ${ }^{+}$leukaemic cells; and by using inducible clones transduced with a dominant negative mutant of Reptin, which contains a point mutation on its ATPase (Walker B) domain, generated from mouse leukaemic cells expressing the MLLENL fusion. In both experiments there was a significant delay in leukaemia progression and, in some cases, a complete remission was achieved. Thus, the results indicated that, at least in mouse cells, Reptin is necessary for leukaemia progression in vivo, probably due to its ATPase activity (PhD thesis, Zhao, 2013). However, since the mouse MLL-ENL ${ }^{+}$leukaemic cells exhibit a number of differences to human AML cells, including a greatly increased rate of proliferation, it was 
important to investigate the dependence of human AML cells on continued Reptin expression in vivo.

In this chapter we have studied the effect of targeting Reptin in established human AML, to resemble a therapeutic setting, by using xenografts in vivo. We did not use cells constitutively expressing a shRNA targeting Reptin, as it would have not been a useful method to assess the consequences of Reptin loss in established leukaemia. For that reason, previous attempts in our laboratory used a heterogeneous bulk population of cells expressing an inducible shRNA targeting Reptin. However, the experiments failed due to the outgrowth of poorly-transduced cells. Therefore, in this study we used inducible clones previously generated in our group from the human MLL-AF9 ${ }^{+}$cell line THP-1 (PhD thesis, Zhao, 2013). Using a clonal population of cells helped us to better address the question of whether Reptin expression is necessary for leukaemia progression, as this time we ensured a uniform and simultaneous response in all cells.

We first validated that effective Reptin protein depletion was still achievable after transduction with a luciferase-expressing vector, and after induction with DOX. We then transplanted NSG mice with the inducible THP1 clones and measured bioluminescence signal in the transplanted mice to monitor leukaemia progression over time. Even though our western blot analyses showed a slight leakiness in the inducible system, we detected similar levels of luciferase signal in all mice 10 days after transplantation, indicating that similar levels of leukaemic burden were achieved in all groups. Remarkably, Reptin depletion after DOX treatment in 
transplanted mice resulted in a striking remission of established leukaemia, as judged by a reduction of luminescence signal to background levels. Complete remission of the disease in 5 out of 6 mice was also observed, and those mice survived until the end of the experiment without any signs of disease. In contrast, established leukaemia progressed in mice transplanted with inducible Ruvbl2 shRNA cells but not induced with DOX, and in mice transplanted with inducible scrambled shRNA cells. These results are in line with a previous study that found a reduction of tumour progression in vivo after Reptin depletion in human hepatocellular carcinoma using a similar inducible system (Menard et al., 2010). Most importantly, our results are also in accordance with previous data obtained in our laboratory using primary mouse leukaemic cells (PhD thesis, Zhao, 2013). In contrast to that study, we did not use primary cells here but a human cell line which could have lost dependency for Reptin after multiple passages in culture. However, our data indicate that the leukaemic cell line used still required Reptin expression to progress in vivo and suggests that Reptin could be a good target for therapy in human AML.

According to our knowledge, no Reptin-specific drugs have been reported so far, although Pontin-specific inhibitors have been developed. In particular, one of the inhibitors targeting the ATPase activity (Walker B domain) of Pontin was cytotoxic in the human promyelocytic leukaemia cell line HL60 (Elkaim et al., 2014). Targeting the ATPase function of Pontin or Reptin could be a good approach in therapy, as several reports have shown that the ATPase activity of Reptin and Pontin is essential to modulate oncogenic transformation. For example, an ATPase-deficient mutant of Pontin inhibited c-MYC or E1A/E2F1 mediated transformation of early- 
passage rat embryo fibroblasts and increased apoptosis (Wood et al., 2000) (Dugan et al., 2002). In a different report, the ATPase activity of Reptin was found to be required for tumour cell growth and viability of hepatocellular carcinoma cells (Grigoletto et al., 2013). Moreover, previous work in our laboratory found that the Walker B domain mutant of Reptin decreased proliferation, increased apoptosis and reduced the clonogenic potential of leukaemic cells (Osaki et al., 2013). Besides this, impairing the ATPase domain of Reptin in in vivo mouse transplantations reduced leukaemia progression, while the same mutant did not affect normal haematopoiesis in colony formation assays of normal mouse bone marrow HPCs (PhD thesis, Zhao, 2013). In relation to this, in a screen looking for mutations affecting humoral immunity, mice carrying a heterozygous mutation in intron 2 of Reptin, which resulted in a frame shift and premature stop codon, were found to have normal B cell development and just some limitations in T-cell development and in some Tdependent antibody responses (Arnold et al., 2012). Even though homozygous mice carrying the mutation in Reptin were not viable, the fact that very few deleterious effects were observed in the haematopoietic compartment of the heterozygous mice suggests that targeting Reptin in AML patients could be a valid therapeutic approach. Further work should be done to address the effects of pharmacologically targeting the ATPase domain of Reptin in normal human haematopoiesis and in established human leukaemia in vivo. The differential effects of expressing the Reptin ATPase domain mutant in normal mouse haematopoietic progenitors and mouse AML cells suggest that generating antagonists of Reptin ATPase activity could be an efficient and novel approach for the treatment of leukaemia without having a major impact on normal haematopoiesis. However, it will be important to evaluate the specificity of 
such targeting strategies, in particular whether any novel drugs would also target the ATPase domain of the homologous Pontin protein. In this context, it is important to note that conditional knockout of Pontin in the haematopoietic compartment led to bone marrow failure (Bereshchenko et al., 2012) (Rajendra et al., 2014).

In conclusion, in this chapter, we have shown that Reptin depletion impairs human leukaemia progression in vivo and that Reptin represents a potential target for therapy. The following chapters addressed the potential mechanisms underlying the dependency of human AML cells on Reptin expression. 


\section{CHAPTER IV - Studying the molecular function of Reptin in human acute myeloid leukaemia}

\subsection{Introduction}

Reptin and Pontin have been found to function in several cellular activities, including DNA damage repair, DNA replication and mitotic spindle assembly, telomerase complex assembly and transcriptional regulation (Jha and Dutta, 2009) (Nano and Houry, 2013). Among the many different roles described, modulation of gene expression is one of the best studied and there are different ways in which Reptin and Pontin perform this function.

On one hand, they were found to be part of several chromatin remodelling complexes that facilitate access to DNA. These include: the Ino80 complex, which catalyses ATP-dependent mobilization of nucleosomes in regions of active transcription, and to the function of which Rvbp1p/Rvbp2p (the homologs of Pontin and Reptin in yeast) were found to be essential (Jonsson et al., 2004); the SWR/SRCAP complex, which catalyses the introduction of the non-canonical variant histone H2A.Z to the nucleosome, which is associated with active transcription and essential for transcriptional regulation; and the NuA4/Tip60/EP400 complex, which has HAT activity at promoters (Nano and Houry, 2013). 
On the other hand, Reptin and Pontin were also found to interact directly with and/or regulate the activity of several transcriptional modulators, sometimes independently from each other and even in an antagonistic manner. Some of the transcription factors, whose activity has been found to be regulated by Reptin and Pontin include c-MYC (Wood et al., 2000), Gata3 (Hosokawa et al., 2013), ATF2 (Cho et al., 2001) and E2F1 (Tarangelo et al., 2015). Taken together, these studies suggest that the transcriptional regulation activity of Reptin and Pontin is essential for multiple cellular pathways.

As we have seen in the previous chapter, Reptin activity is crucial for human AML survival in vitro (Osaki et al., 2013) and for disease progression in vivo. Moreover, a previous study in our group found a decrease in c-MYC protein after Reptin depletion in a human AML cell line ( $\mathrm{PhD}$ thesis, Zhao, 2013). For these reasons in this study we aimed to investigate the molecular function of Reptin in human AML, and hypothesised that this dependency for Reptin in AML cells could be linked to its role in modulating gene transcription. To that aim, we first examined the effect of Reptin and other known Reptin-interacting partners on the transcription factor c-MYC. In addition, to get a broader understanding of the pathways regulated by Reptin in AML, we also generated global gene expression data following Reptin silencing in human leukaemic cells. 


\subsection{Results}

\subsubsection{Examining the effect of Reptin depletion and depletion of Reptin-interacting}

partners on c-MYC

As mentioned in the introduction, Reptin has been documented to form part of different complexes and to interact with several proteins, including the oncoprotein c-MYC (Wood et al., 2000) (Bellosta et al., 2005). Several c-MYC cofactors have been described, which allow c-MYC to act as a transcriptional activator (for example, MAX cofactor in the c-MYC/MAX complex) or as a transcriptional repressor (for example, the MIZ-1 cofactor in the c-MYC/MIZ-1 complex) (Dang, 2012).

A previous study in our laboratory examined c-MYC protein after Reptin depletion and showed a reduction in c-MYC that was apparent at the protein, but not RNA, level (PhD thesis, Zhao, 2013). The c-MYC decrease was observed in the MLL-rearranged AML cell lines THP-1, Nomo-1 and MV4-11 (PhD thesis, Zhao, 2013). As several publications also suggested a link between Reptin and c-MYC, we decided to examine the possibility that Reptin modulates c-MYC protein function in AML. We first validated the decrease in c-MYC protein levels in the THP-1 clones used in a previous study (PhD thesis, Zhao, 2013) (Figure 17). Next, we validated these results in the bulk population of THP-1 cells. Western blot results showed a decrease in c-MYC after Reptin loss, although this decrease was only detected at a later time point than complete Reptin depletion (Figure 18). The data also showed that c-MYC protein was almost completely lost by day 6 after DOX induction. To a lesser extent, the c-MYC cofactor MIZ-1 had also decreased after 6 days of Reptin 
depletion (Figure 18). Notably, protein levels of the upstream $c-M Y C$ transcriptional regulator c-MYB, remained unchanged (Figure 18). Overall, these results indicate that Reptin modulates the stability of c-MYC and MIZ-1 proteins but that this is not a general proteolysis effect, since c-MYB protein, which also has a very short halflife, did not change.

An earlier study from our group had already shown the deleterious effect of transducing AML cells with a dominant negative (D/N) mutant of Reptin (Osaki et al., 2013). This mutant carries a point mutation in the conserved Walker B domain (Reptin (D299N)), which impairs its capacity to bind DNA and hydrolase ATP (Mézard et al., 1997). In order to investigate the effect of the D/N mutant on c-MYC expression, a bulk population of THP-1 cells was transduced with the D/N mutant of Reptin. As expected, there was a significant increase in apoptosis levels in cells transduced with the $\mathrm{D} / \mathrm{N}$ mutant 8 days after transduction, as compared to cells transduced with a control empty vector (EV) (Figure 19 A), confirming that the ATPase activity of Reptin is essential for the effect on viability observed upon Reptin knockdown. Interestingly, western blot analysis also showed a stable and significant decrease in c-MYC protein in cells transduced with the D/N mutant of Reptin (Figure 19 B and C), suggesting that the ATPase activity of Reptin is essential for its effect on c-MYC.

In order to examine the mechanism by which Reptin exerts its function, we decided to examine the effect of inhibiting known Reptin interacting partners on cMYC protein. One of these partner proteins, that Reptin and Pontin can both interact 
with, is the oncogenic protein, MTPB. Interestingly, MTBP was also found to be necessary to increase c-MYC mediated transcription, proliferation and neoplastic transformation (Grieb et al., 2014). As Reptin has been proposed to act as a chaperone-like protein, helping in the assembly of several complexes (Nano and Houry, 2013), we explored the possibility that the apoptotic phenotype observed after Reptin depletion was the result of a failed interaction or assembly of the MTBP/cMYC complex. In order to test this hypothesis, we examined the effect of MTBP depletion on c-MYC protein stability in THP-1 cells. Cells transduced with 4 different shRNA targeting MTBP showed reduced levels of c-MYC protein compared to cells transduced with a non-targeting shRNA, while Reptin protein levels did not change (Figure 20A and Figure 20B). This experiment indicated that MTBP loss is enough to induce c-MYC protein degradation. Interestingly, Reptin loss in one of the inducible THP-1 clones also led to a decrease of MTBP protein (Figure 20C). As Reptin has been described to be necessary for the formation of some transcriptional complexes and it was found to interact with both MTBP and cMYC, it is plausible that the reason behind c-MYC and MTBP protein degradation after Reptin loss could be the failed complex formation or interaction between cMYC and MTBP in AML cells.

Reptin has also been found to form part of the EP400 complex and interact with some of the proteins of the complex (Fuchs et al., 2001). EP400 is a multiprotein chromatin remodelling complex whose central component is a $400 \mathrm{kDa}$ protein. Other components of the complex include: the ATPases Reptin and Pontin; a c-MYC associated cofactor called TRRAP/PAF400; $\beta$-actin and the actin-like protein 
BAF53; and the mammalian orthologs of the Drosophila melanogaster Enhancer of Polycomb, EPC1 and EPC2 (Fuchs et al., 2001). Surprisingly, knockdown of EP400 complex components in a recent study (including EPC1, EPC2, Reptin and Pontin), did not decrease but resulted in the accumulation of c-MYC protein and upregulation of c-MYC module genes in THP-1 cells (Huang et al., 2014). As these data differ from our previous results, we decided to assess the effect of EPC1 and EPC2 depletion on c-MYC protein in our THP-1 cells. Western blot analysis of THP-1 cells transduced with different shRNA targeting EPC1 and EPC2 also showed a decrease in c-MYC protein levels and an increase in apoptosis levels (Figure 21), compared to non-targeting vector control transduced cells. This result is consistent with our previous data showing a decrease of c-MYC after Reptin depletion, but differs from the results obtained by Huang et al.

Taken together, the results described above pointed to a possible regulation of c-MYC by Reptin. However, it was still not clear whether this was at the protein level or at the transcriptional level. A previous study in our laboratory examined the mRNA levels of $c-M Y C$ after Reptin depletion and did not find a significant change in the $c-M Y C$ transcript after Reptin depletion (PhD thesis, Zhao, 2013). However, the data were obtained from a single experiment and the level of Reptin knockdown achieved in that experiment was much lower than the one achieved in the present study. Therefore, we decided to re-examine $c-M Y C$ transcript levels after Reptin knockdown in THP-1 cells. Surprisingly, our qRT-PCR results indicated a reduction in $c-M Y C$ mRNA levels after 4 days of Reptin knockdown (Figure 22). Thus, it was plausible to hypothesise that Reptin loss may principally impact on the 
transcriptional regulation of $c-M Y C$, rather than affecting the assembly of c-MYC complexes. 


\section{C2 shSCR clone}

\section{$\frac{\text { Day } 1}{-+} \frac{\text { Day } 2}{-+} \frac{\text { Day } 3}{-+} \frac{\text { Day } 4}{-+} \frac{\text { Day } 5}{-+} \frac{\text { Day } 6}{-+}$}

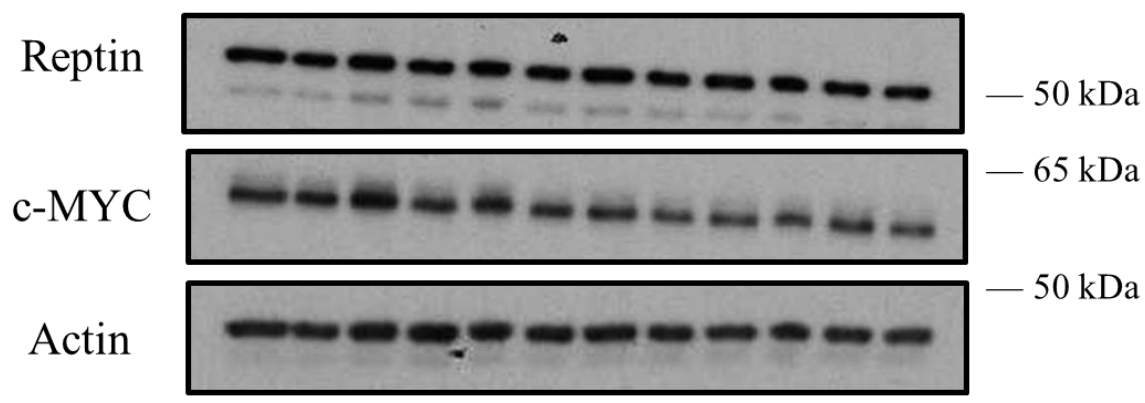

R6 shRep clone

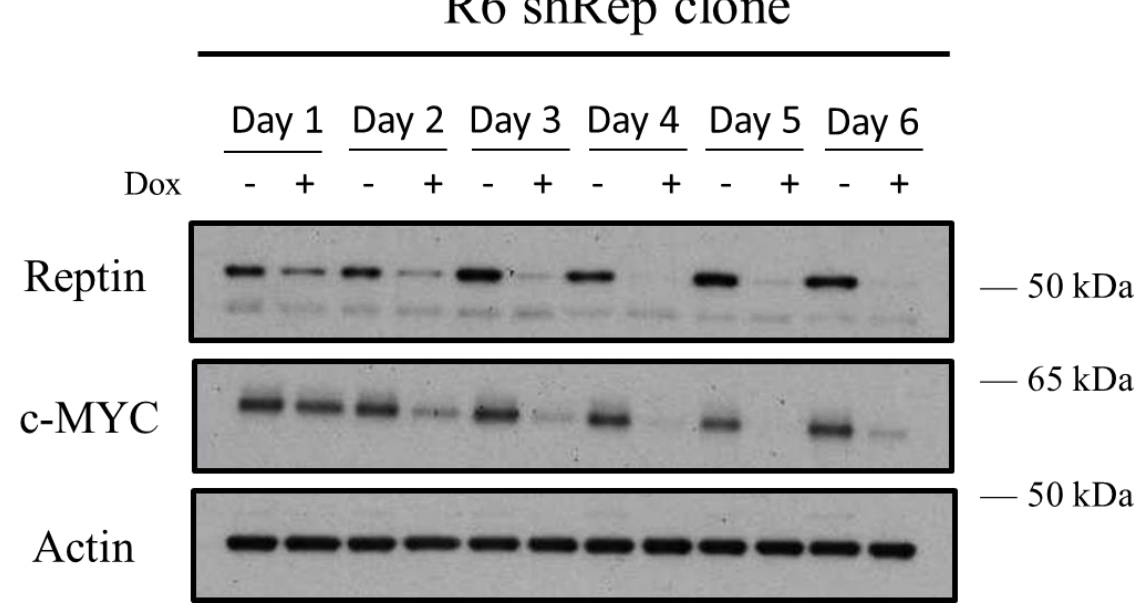

Figure 17. Inducible Reptin depletion leads to decreased c-MYC protein in a THP-1 clone.

Western blot analysis of Reptin, c-MYC and Actin protein after treating a scrambled shRNA (C2 shSCR) clone and Reptin shRNA (R6 shRep) clone, generated from THP-1 cells, with or without DOX for 1-6 days. Actin was used as a loading control. This figure is a representative example of two independent experiments. 
A)

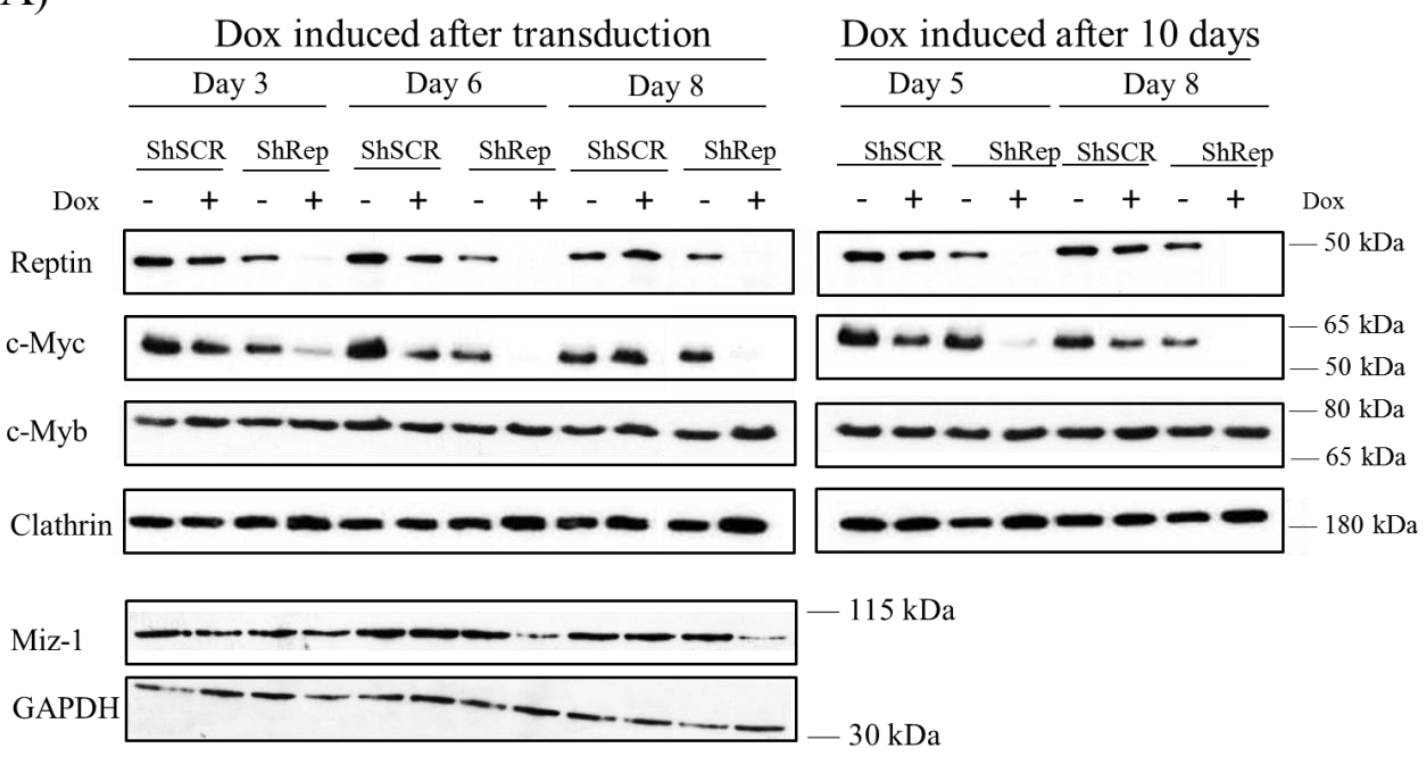

B)

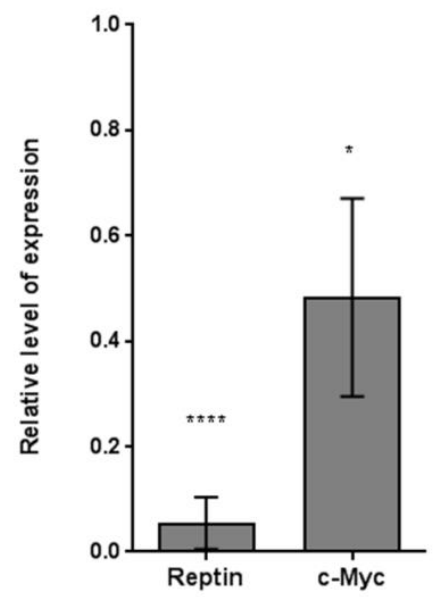

Figure 18. Reptin knockdown leads to c-MYC and Miz-1 loss.

(A) Western blot analysis of Reptin, c-MYC, c-MYB and MIZ-1 protein in DOX inducible scrambled shRNA (shSCR) and Reptin shRNA (shRep) transduced THP-1 cells (bulk population). Cells were transduced and selected with puromycin for 3 days. Immediately after, cells were treated with or without DOX for 3, 6 and 8 days (left panel). Some cells were left untreated for 10 days after puromycin selection and 
then treated with or without DOX for 5 and 8 days (right panel). Clathrin and GAPDH were used as a loading control. The data are from of a single experiment. (B) Reptin and c-MYC protein levels at day 4 in DOX-induced cells relative to NonDOX induced control cells normalised to 1, as quantified by the Quantity One software and normalised for the loading control Clathrin. Bars represent the mean of five independent experiments and error bars represent SD. $P$-values comparing normalised -DOX to +DOX samples were calculated using the one-sample $t$-test, $(*)$ $p \leq 0.05 ;(* * * *) p \leq 0.0001$. 
A)

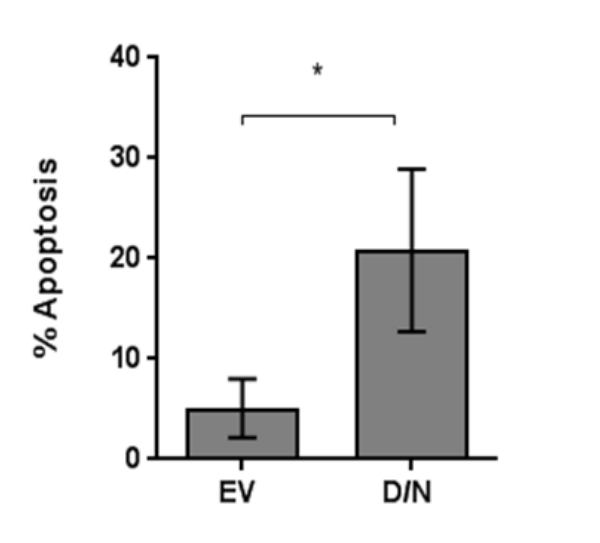

B)

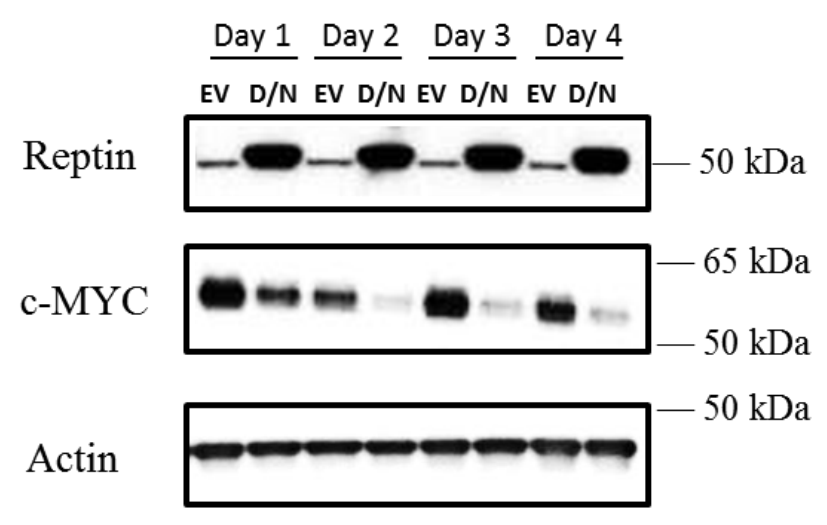

C)

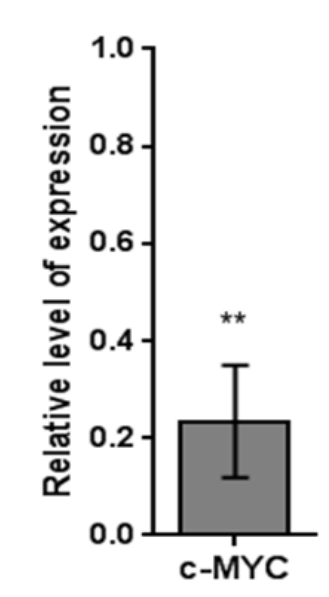

Figure 19. Overexpression of a Dominant Negative $(\mathrm{D} / \mathrm{N})$ mutant of Reptin leads to apoptosis and c-MYC protein loss.

(A) Bar chart showing quantification of apoptosis in THP-1 cells overexpressing the Reptin (D299N) Dominant Negative (D/N) mutant. Bars represent the mean of three independent experiments and error bars represent SD. $P$-value was calculated using unpaired Student's $t$-test, $\left({ }^{*}\right) p \leq 0.05$. (B) Western blot analysis of Reptin and c-MYC proteins in THP-1 cells transduced with the Reptin (D299N) D/N mutant, or control empty vector (EV) at the indicated time points. Actin was used as a loading control. (C) c-MYC protein levels from (B) of cells expressing the D/N mutant of Reptin for 4 days relative to Empty Vector (EV) control expressing cells normalised to 1, as 
quantified by the Quantity One software and normalised for the loading control Actin. Bars represent the mean of three independent experiments and error bars represent SD. A p-value comparing c-MYC expression in EV (normalised to 1) and $\mathrm{D} / \mathrm{N}$ mutant expressing cells was calculated using the one-sample $t$-test, $\left({ }^{* *}\right) p \leq 0.01$. 
A)

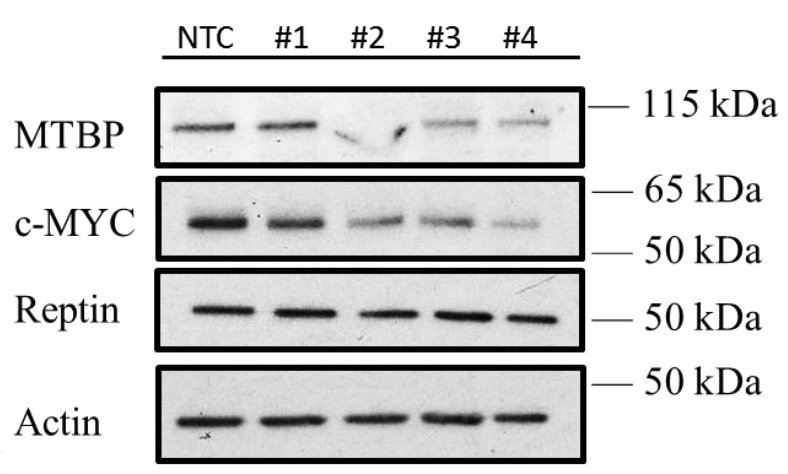

B)

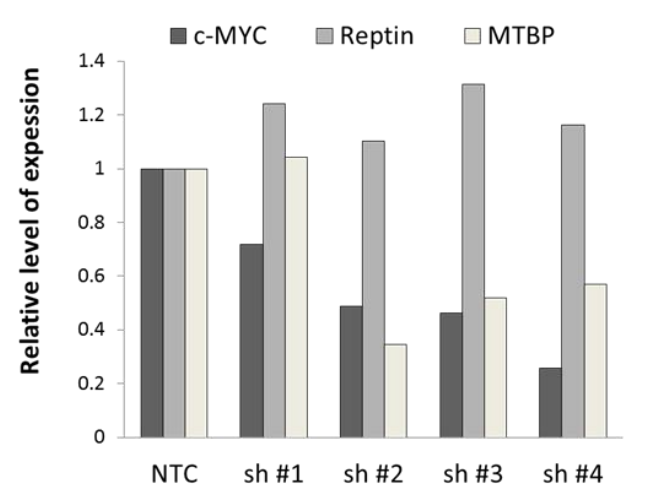

C)

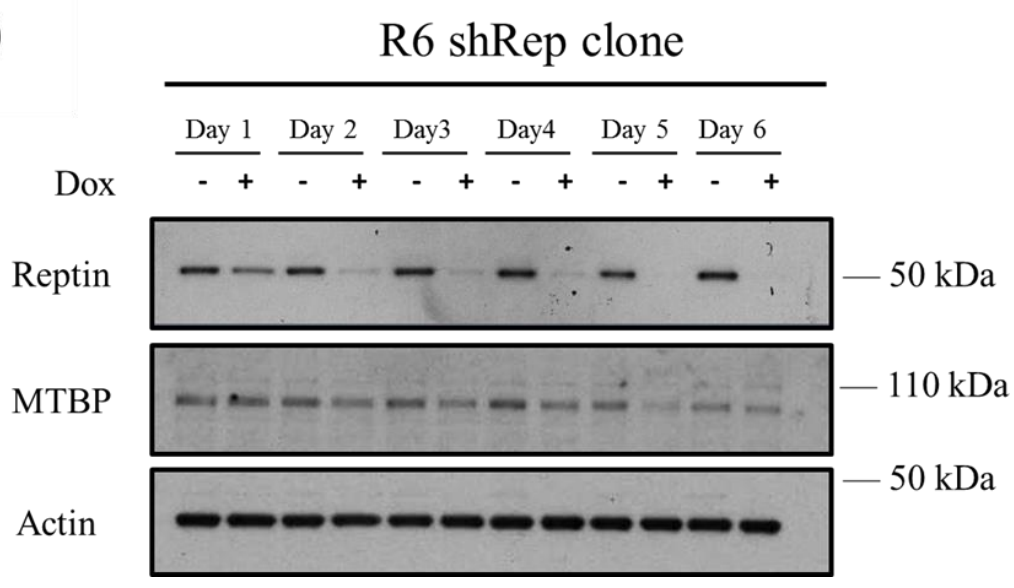

Figure 20. MTBP knockdown induces c-MYC protein degradation.

(A) Western blot analysis of MTBP, Reptin, c-MYC and Actin proteins in THP-1 cells transduced with 4 different pGIPZ lentiviral shRNA vectors targeting MTBP (\#1, \#2,\#3 and \#4). Actin was used as a loading control. These data were obtained 
from a single experiment. (B) c-MYC, Reptin and MTP protein levels from (A) relative to the Non Targeting Control (NTC) treated cells normalised to 1 , as quantified by the Quantity One software and normalised for the loading control Actin. (C) Western blot analysis of MTBP, Reptin and Actin following treatment with or without DOX for 1-6 days in the inducible Reptin shRNA (R6 shRep) THP-1 clone. Actin was used as a loading control. These data were obtained from a single experiment. 
A)

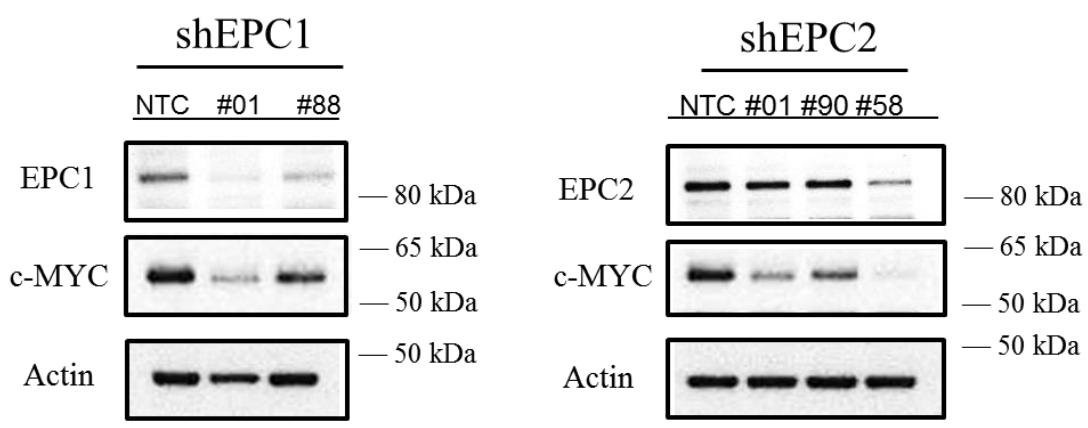

B)
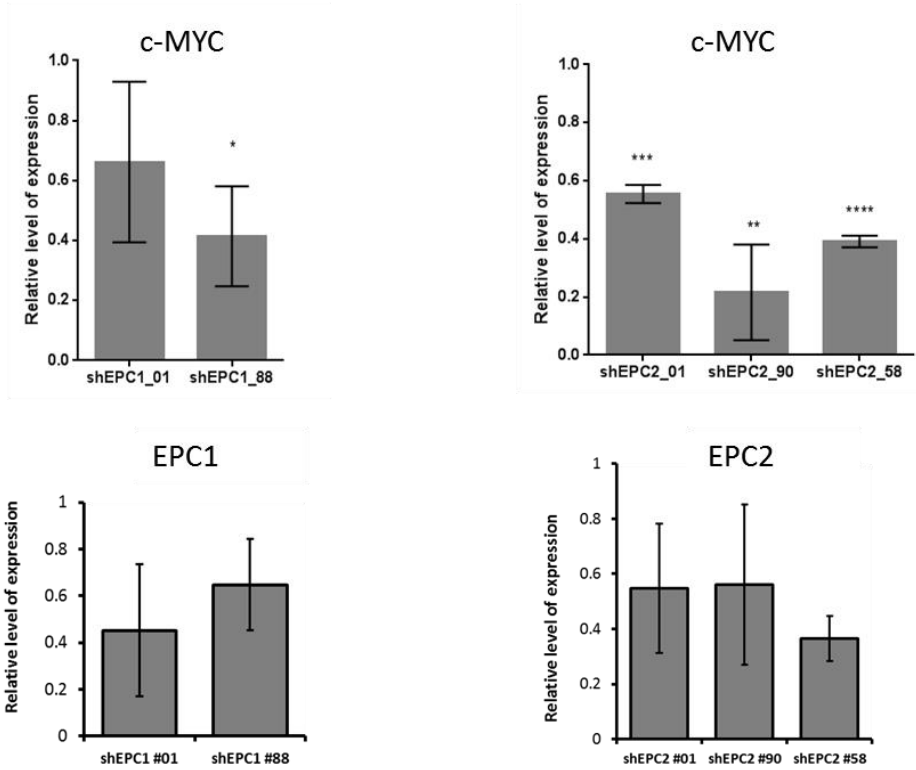

C)
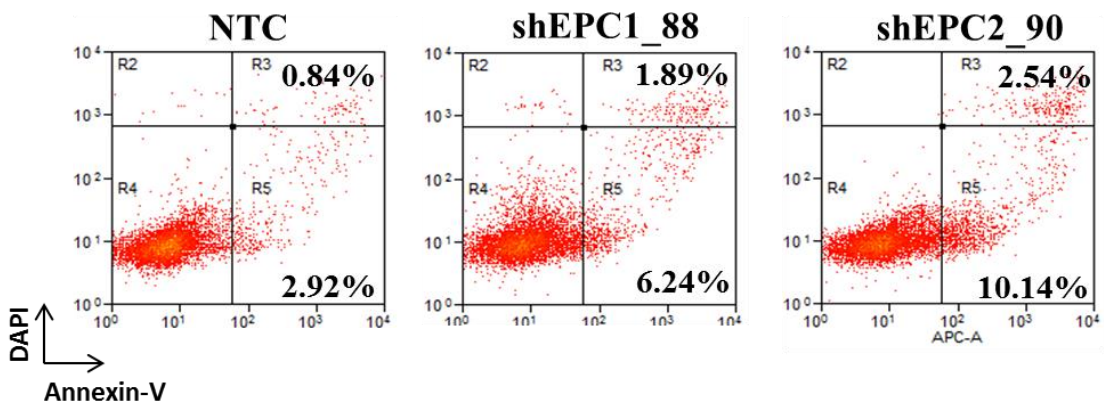

Figure 21. Knockdown of EPC1 and EPC2 induces c-MYC loss in THP-1 cells.

(A) Western blot analysis of EPC1, EPC2 and c-MYC proteins in THP-1 cells transduced with different pLKO.1 lentiviral shRNA vectors targeting EPC1 (\#01 and \#88) and EPC2 (\#58, \#01 and \#90) or a Non Targeting Control (NTC). Actin was 
used as a loading control. (B) c-MYC, EPC1 and EPC2 protein levels from (A) relative to Non-Targeting control (NTC) transduced control cells normalised to 1 , as quantified by the Quantity One software and normalised for the loading control Actin. Bars represent the mean of three (MYC) or two (EPC1 and EPC2) independent experiments and error bars represent SD. $P$-values comparing normalised NTC to shRNA expressing samples were calculated using one-sample ttest, $(*) p \leq 0.01(* *) ; p \leq 0.01 ;(* * *) p \leq 0.001 ;(* * * *) p \leq 0.0001$. (C) Flow Cytometry plots showing apoptosis for transduced cells in (A). The apoptosis data were obtained from of a single experiment. 
A)
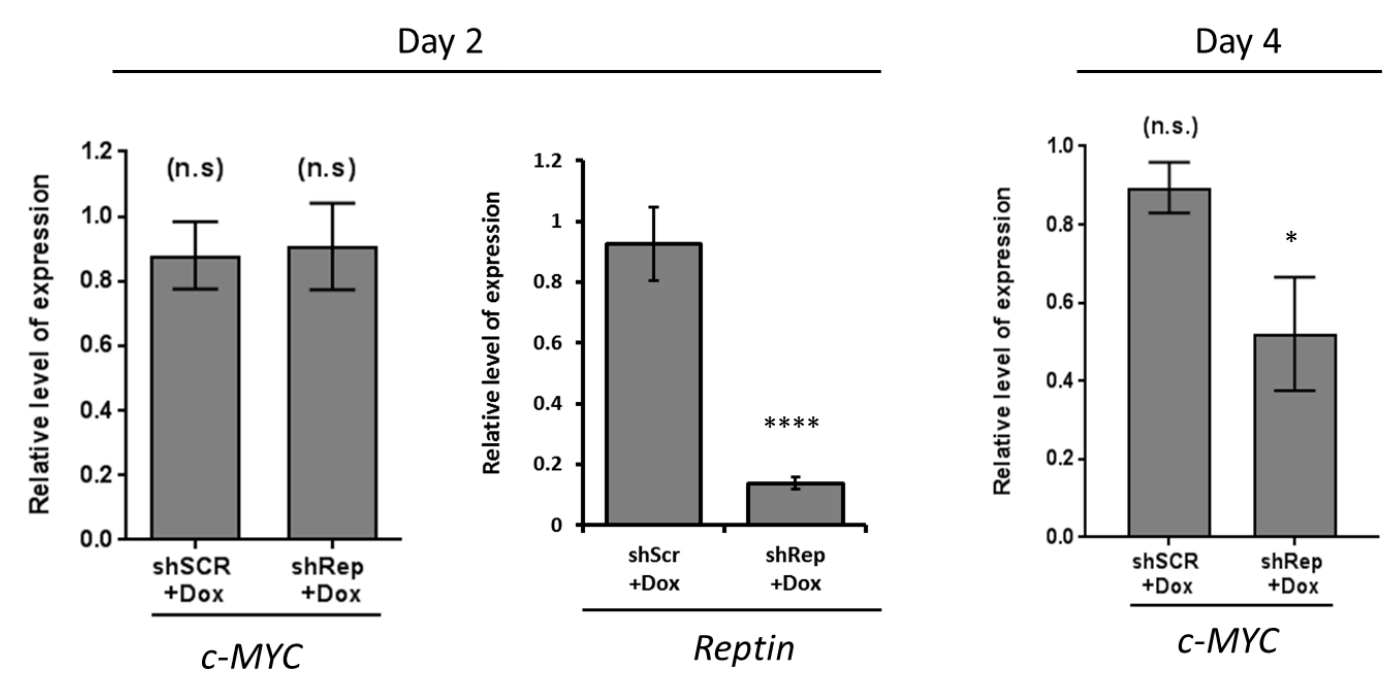

B)
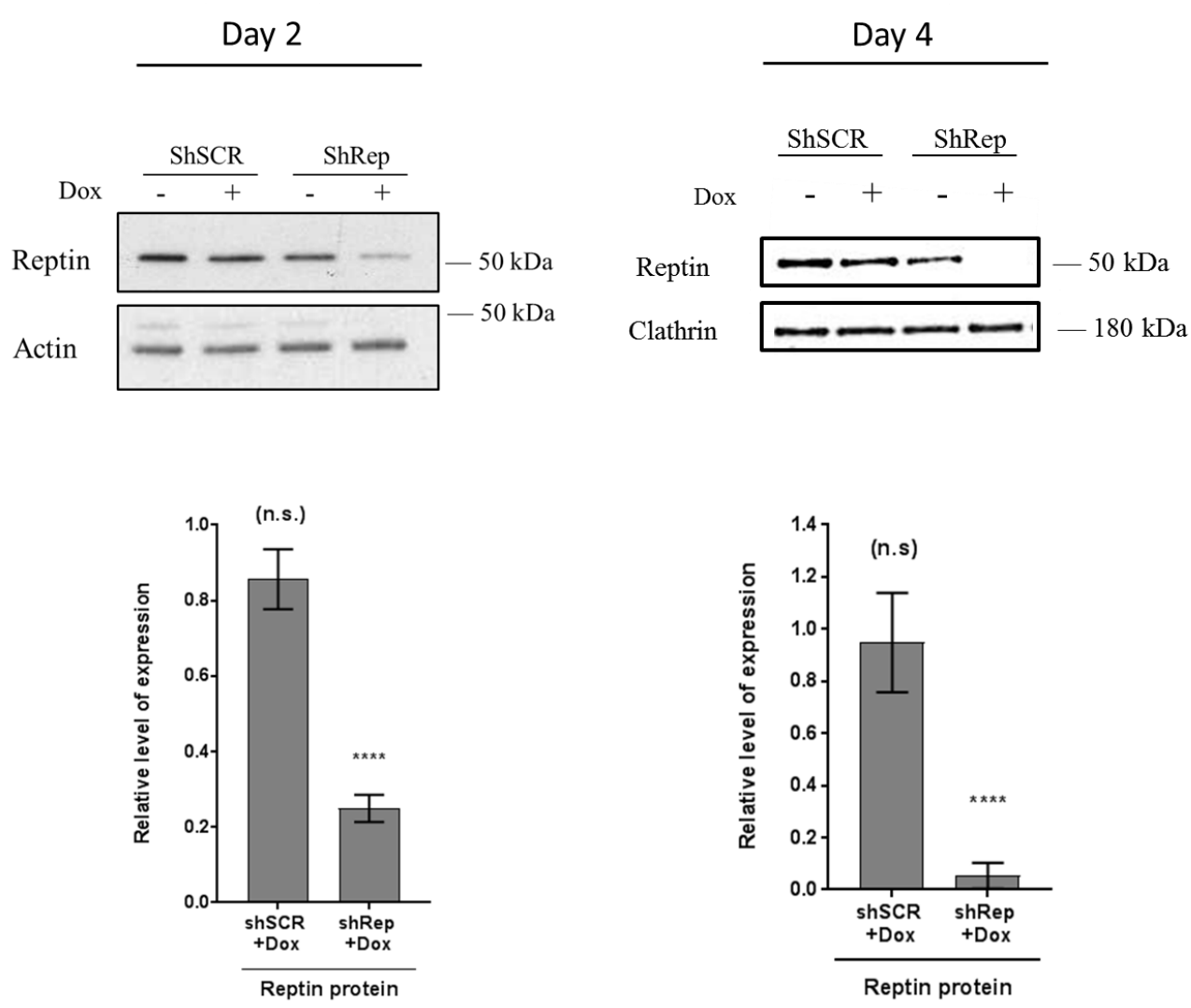

Figure 22. c-MYC transcript levels decrease after Reptin depletion.

(A) Plots represent qRT-PCR analysis of $c-M Y C$ and Reptin mRNA levels from THP-1 cells transduced with an inducible control shRNA (shSCR) or a shRNA 
targeting Reptin (shRep) after 2 days or 4 days of DOX induction. The data are relative to non-DOX (-DOX) induced cells normalised to 1. (B) The figure shows Reptin protein levels of cells used in (A) after 2 and 4 days of induction with or without DOX. The data were quantified by the Quantity One software and normalised for the loading control. Columns represent the mean of three (day 2) or five (day 5) independent experiments and error bars, the SD. $P$-values comparing normalised $-\mathrm{DOX}$ to $+\mathrm{DOX}$ samples were calculated using the one-sample $t$-test; $(* * * *) p \leq 0.001 ;(* * *) p \leq 0.001 ;(*) p \leq 0.05 ;$ (n.s.) not significant $(p \geq 0.05)$. 


\subsubsection{Reptin does not interact with c-MYC in AML cells}

To elucidate the underlying molecular mechanism by which Reptin modulates cMYC function, we performed immunoprecipitation assays to examine whether complexes between Reptin and c-MYC can be detected in THP-1 cells. To that aim, we first generated a 3xHA-tagged version of Reptin and transduced bulk THP-1 cells to overexpress the tagged protein. We then confirmed the overexpression of the tagged protein in THP-1 cells by western blot analysis (Figure 23). Next, we performed immunoprecipitation assays and detected Reptin when we used an antiHA antibody to immunoprecipitate the lysates, but not when we used a control antiIgG antibody (Figure 24), confirming the specificity of the antibodies. Moreover, we were also able to co-immunoprecipitate Pontin with the 3xHA-tagged Reptin, which suggested that the 3xHA-tagged version of Reptin was still functional and capable of forming complexes with its close related partner protein (Figure 24). However, after immunoprecipitating 3xHA-Reptin overexpressing cells with an anti-HA antibody we were unable to detect endogenous c-MYC co-immunoprecipitating with the 3xHA-tagged Reptin (Figure 25). These data are consistent with our hypothesis that Reptin may affect c-MYC function in THP-1 cells by regulating its $c-M Y C$ gene transcription, rather than assembly of c-MYC containing complexes. In order to investigate this hypothesis further, and to have a broader understanding of the impact of Reptin on gene transcription in AML, we decided to generate global gene expression data after Reptin depletion. 


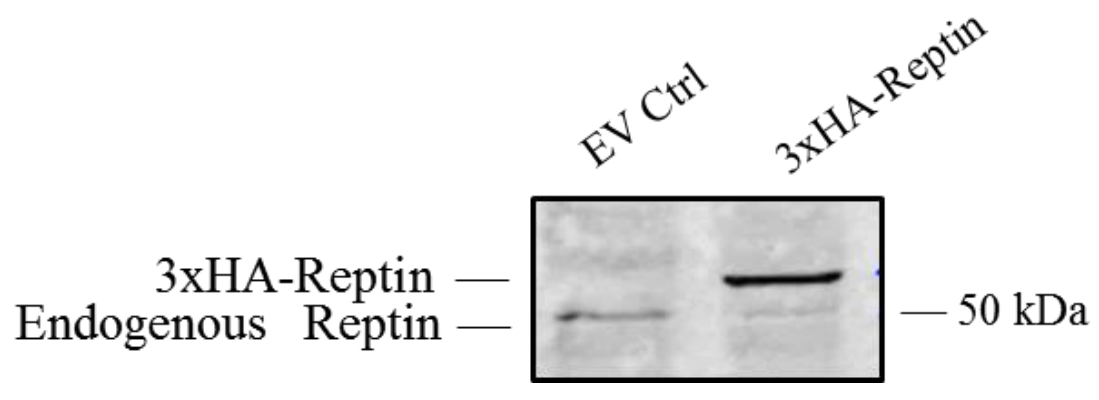

Figure 23. Western blot analysis showing 3xHA-tagged Reptin overexpression.

The figure displays western blot analysis of Reptin protein. THP-1 cells were transduced with a control empty vector (EV Ctrl) or with the 3xHA-tagged Reptin construct and selected with puromycin for 3 days. Lysates were then subjected to western blot analysis to verify overexpression and detection of the 3xHA-tagged Reptin protein. This experiment was performed once. Endogenous Reptin was used as a loading control. 
A)

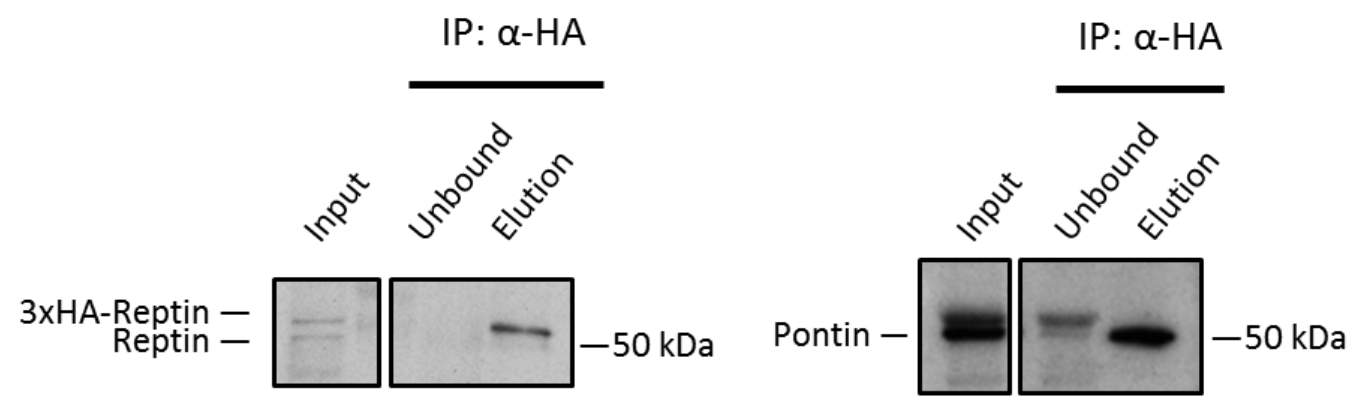

B)

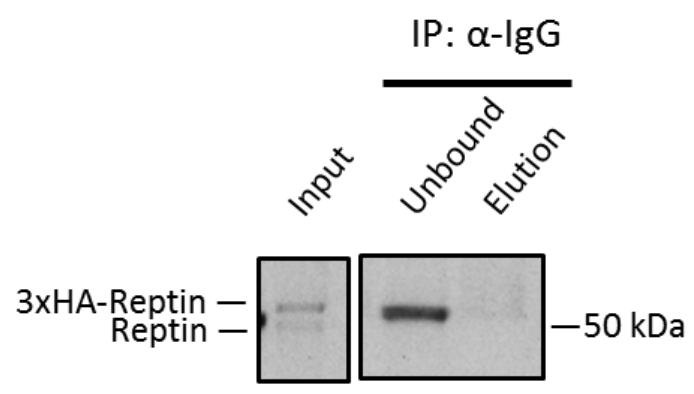

Figure 24. 3xHA-Reptin interacts with Pontin in THP-1 cells.

The figure displays western blot analysis of 3xHA-tagged Reptin protein and shows the interaction with endogenous Pontin. A 3xHA-tagged Reptin plasmid was overexpressed in THP-1 cells and whole cell lysates of puromycin selected cells were immunoprecipitated with an antibody against the HA tag (A) or control IgG (B). The resultant precipitates were immunoblotted with anti-Reptin or anti-Pontin antibodies. "Input" refers to the total whole lysate. "Elution" refers to amount of protein co-immunoprecipitated by the specific antibody, whereas "Unbound" refers to the amount of protein not co-immunoprecipitated. The data are representative of two independent experiments. 


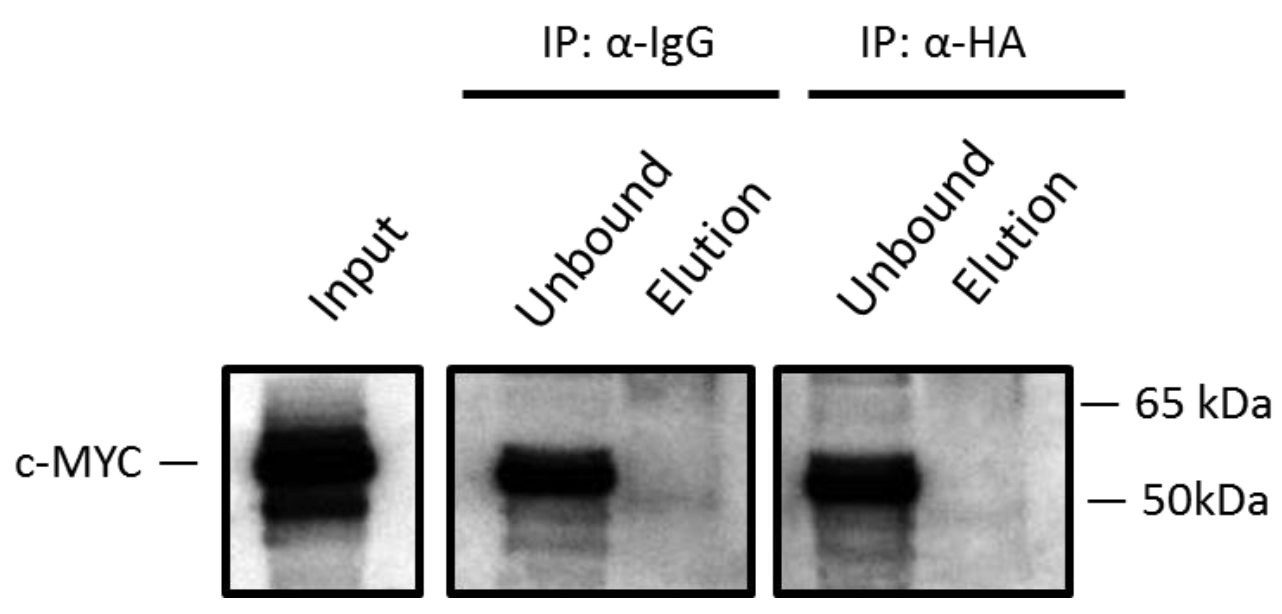

Figure 25. 3xHA-tagged Reptin does not interact with c-MYC

A 3xHA-tagged Reptin plasmid was overexpressed in THP-1 cells and whole cell lysates of puromycin selected cells were immunoprecipitated with an antibody against the HA tag or control IgG. The resultant precipitates were immunoblotted with an anti-c-MYC antibody. "Input" refers to the total whole lysate. "Elution" refers to amount of protein co-immunoprecipitated by the specific antibody, whereas "Unbound" refers to the amount of protein not co-immunoprecipitated. The data are representative of three independent experiments. 


\subsubsection{Characterising the response of bulk THP-1 cells to Reptin loss}

For transcriptome profiling we used bulk populations of the inducible human AML cell line THP-1, instead of the previously described clones. This was done to avoid any clone-specific gene expression patterns that may not reflect those in the bulk culture, and because the planned analyses were performed over a relatively short time frame. Thus, we first validated that efficient inducible Reptin knockdown in the bulk population was possible. We transduced the bulk of THP-1 cells with the inducible "Tet-On" lentiviral expression vector described in the previous chapter, containing a Reptin-specific shRNA, or a control scrambled-shRNA. Treatment of the transduced cells with DOX resulted in efficient induction of the shRNA in the bulk population, as shown by the high levels of tRFP expression detected by flow cytometry as early as $24 \mathrm{~h}$ after DOX addition (Figure 26A). Western blot analyses revealed efficient Reptin depletion, protein being undetectable after 3 days of DOX treatment (Figure 26B, left panel). Moreover, flow cytometry analyses showed an increase in apoptosis 8 days after Reptin knockdown (Figure 26C). Similarly to what we had observed in the clones, our western blot results with bulk THP-1 cells indicated leakiness in the system. However, we did not detect a substantial increase in apoptosis in the shReptin -DOX samples in comparison to the control -DOX ones (Figure 26C). In addition, Reptin protein depletion and an increase in apoptosis in Reptin depleted samples were also observed upon DOX induction when using cells that had been grown for 10 days in culture, following transduction (Figure 26B and Figure 26C, right panels). Thus, shRNA inducibility in the bulk transduced THP-1 cells appeared relatively stable in short-term culture. 
Since we observed a significant response to DOX treatment in the bulk THP1 cells, we then aimed to identify the best time point for gene expression analysis. We wanted to identify the earliest time point that would allow enough time for primary gene expression changes due to Reptin loss to occur, without this data being obscured by secondary gene expression changes associated with cell differentiation and apoptosis. In order to identify the best time point, we repeated the previous western blot experiments in a time course manner, taking protein lysates every $24 \mathrm{~h}$. We then chose time point Day 2 for RNA-seq analysis, as it was the earliest time point in which Reptin depletion was observed by western blot, and a later time point (Day 4) at which Reptin depletion peaked (Figure 27).

We then proceeded to perform the experiments at the chosen time points for use in RNA-seq analysis. The bulk population of THP-1 cells was transduced with the inducible vector containing a Reptin-specific shRNA, or a control scrambledshRNA, and induced with DOX in multiple independent experiments in order to generate enough replicates to submit for sequencing analysis. Protein lysates and RNA were taken at days 2 and 4 after DOX induction, and western blot analyses were performed to verify efficient Reptin depletion. The average level of Reptin knockdown at day 2 achieved in 3 different experiments was 75\% (Figure 28) and at day 4 (data from 5 different experiments) was 95\% (Figure 29). The RNA from all from these experiments at day 2 and 4 was then extracted and submitted for sequencing analysis. As we wanted to make sure that our gene expression results were going to be meaningful, we also left a few cells in culture in the presence of DOX and measured apoptosis levels after 8 days in culture. Our results showed an 
increase in apoptosis of more than 5-fold in the Reptin depleted samples, reaching apoptosis levels of around $20 \%$, as compared to the non-DOX induced samples in which apoptosis levels were less than 5\% (Figure 29). With this result we ensured that the cells used for gene expression analysis responded as expected to Reptin depletion. 
A)
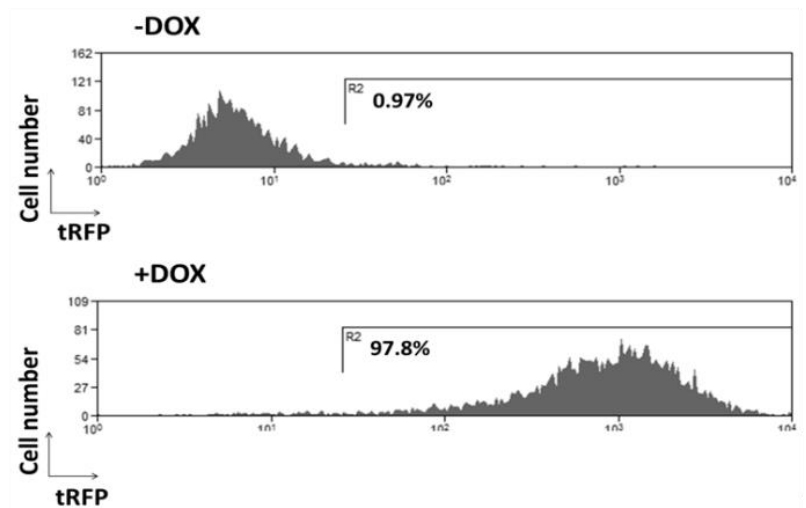

B)

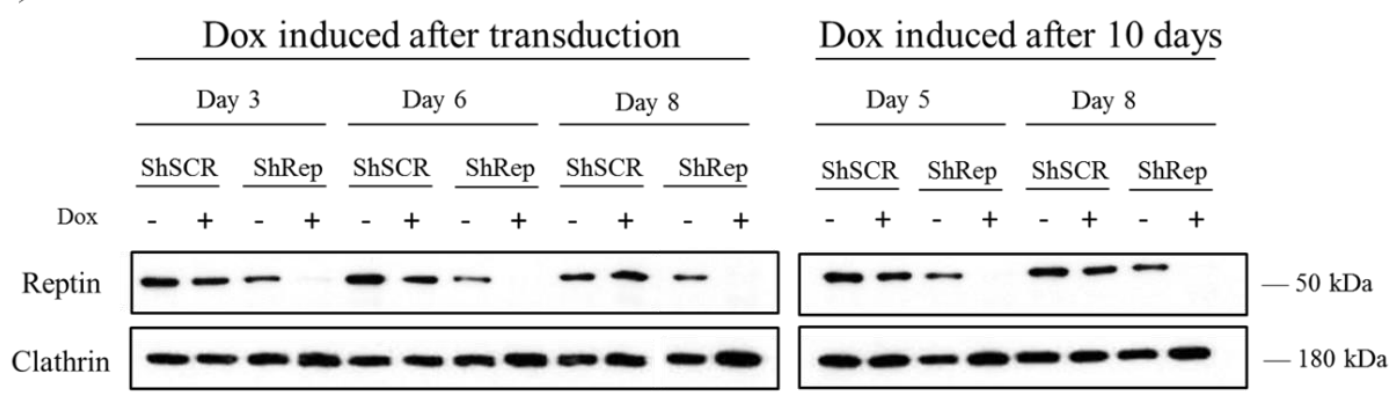

C)
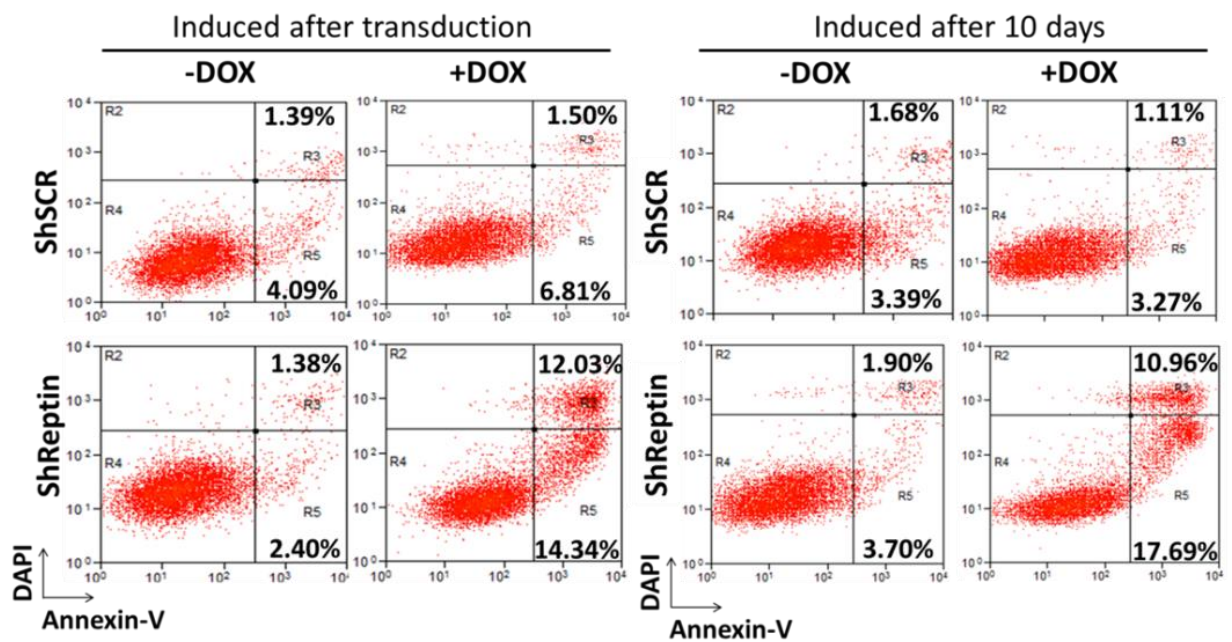

Figure 26. Inducible knockdown of Reptin in a bulk population of THP-1 cells

(A) The plots represent examples of flow cytometric analysis of tRFP expression in inducible Reptin shRNA transduced THP-1 cells (bulk population) $24 \mathrm{~h}$ after DOX 
induction. (B) Western blot analysis of Reptin protein in inducible scrambled shRNA (shSCR) and Reptin shRNA (shRep) transduced THP-1 cells (bulk population), following treatment with or without DOX. In the left panel, cells were treated with DOX immediately after transduction every $48 \mathrm{~h}$, and protein lysates were taken 3,6 and 8 days after the first DOX treatment. The right panel shows cells that were treated with DOX 10 days after transduction. Protein lysates were taken 5 and 8 days after the first DOX treatment. Clathrin was used as a loading control. This experiment was performed once. (C) Plots shows an example of apoptosis levels measured by flow cytometric analysis of Annexin V and DAPI stained transduced cells from (B) at Day 8 after DOX induction (left panel) or at Day 8 in cells grown for 10 days after transduction and before DOX induction (right panel). 
A)

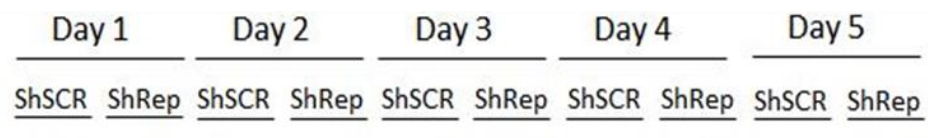
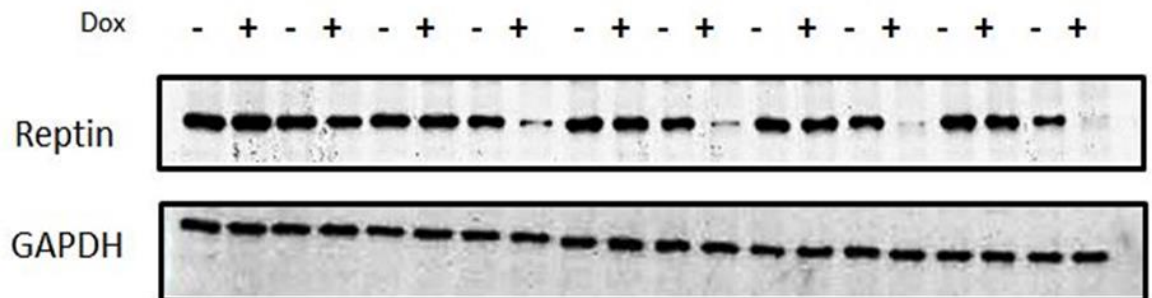

B)

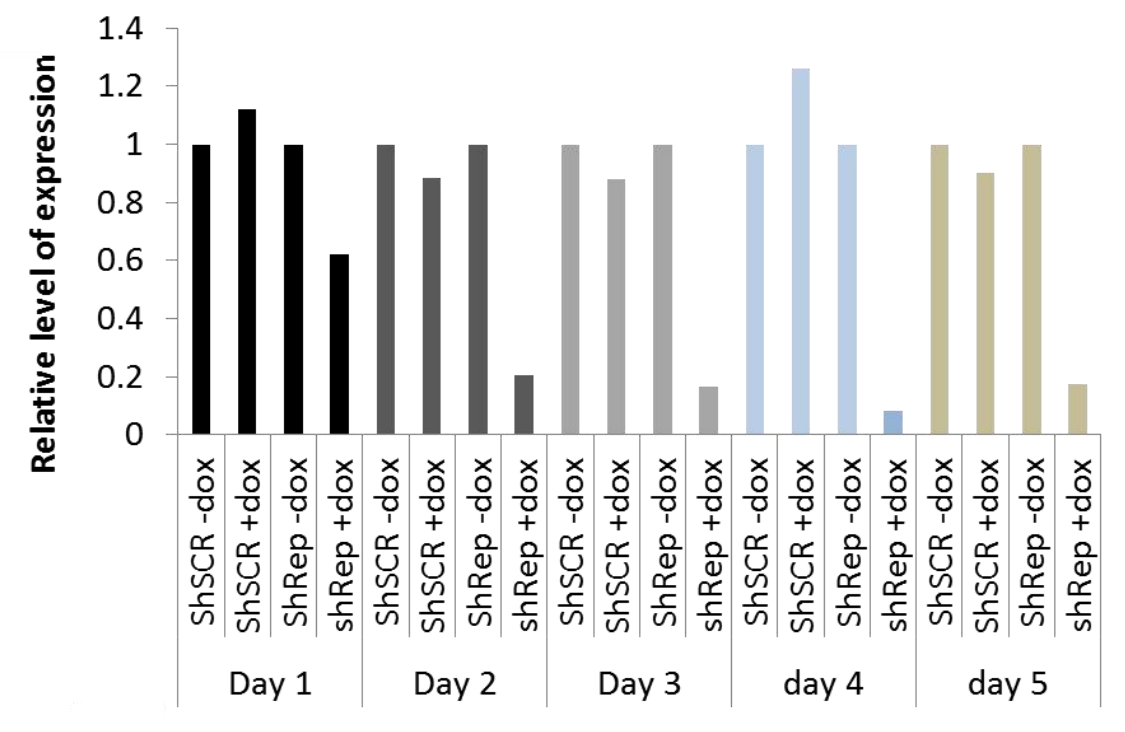

Reptin protein

Figure 27. Time-course western blot analysis of Reptin protein.

(A) Time course of Reptin protein analysed by western blotting, in doxycycline (DOX) inducible scrambled shRNA (shSCR) and Reptin shRNA (shRep) transduced THP-1 cells following treatment with or without DOX for 5 days. GAPDH was used as a loading control. These data were obtained from a single experiment. (B) Reptin protein levels from (A) relative to non-DOX induced control cells normalised to 1 , as quantified by the Quantity One software and normalised for the loading control GAPDH. Data are from a single experiment. 
A)

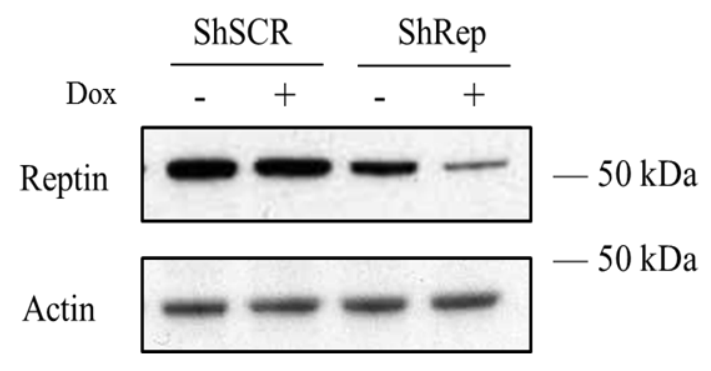

B)

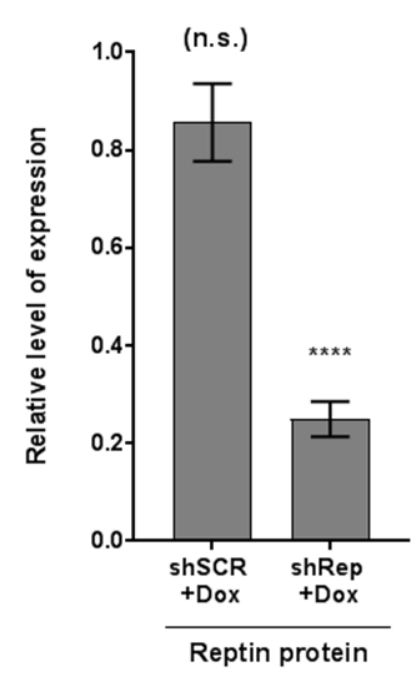

Figure 28. Characterisation of the experiments used for RNA-sequencing at time point Day 2.

(A) Western blot analysis of Reptin protein in DOX inducible scrambled shRNA (shSCR) and Reptin shRNA (shRep) transduced THP-1 cells following treatment with or without DOX for 2 days. Actin was used as a loading control. (B) Reptin protein levels relative to non-DOX (-DOX) treated control cells normalised to 1 , as quantified by the Quantity One software and normalised for the Actin loading control. Bars represent the mean of three independent experiments and error bars represent SD. $P$-values comparing normalised $-\mathrm{DOX}$ to $+\mathrm{DOX}$ samples were calculated using the one-sample $t$-test; $(* * * *) p \leq 0.0001$; (n.s.) not significant ( $p \geq$ 0.05). For clarity purposes, these results have been also shown on figure 22 . 
A)

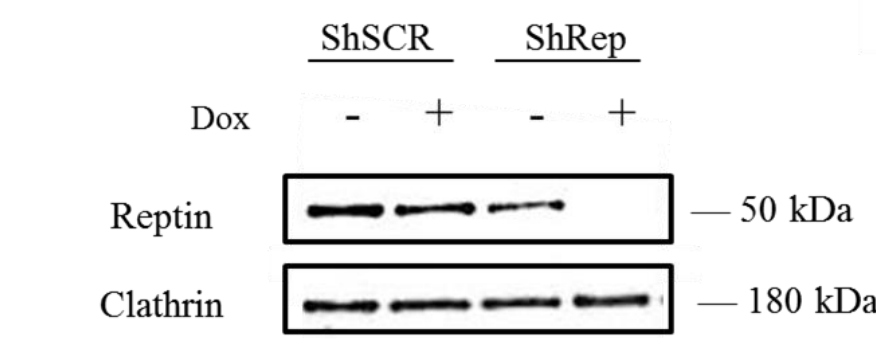

C)
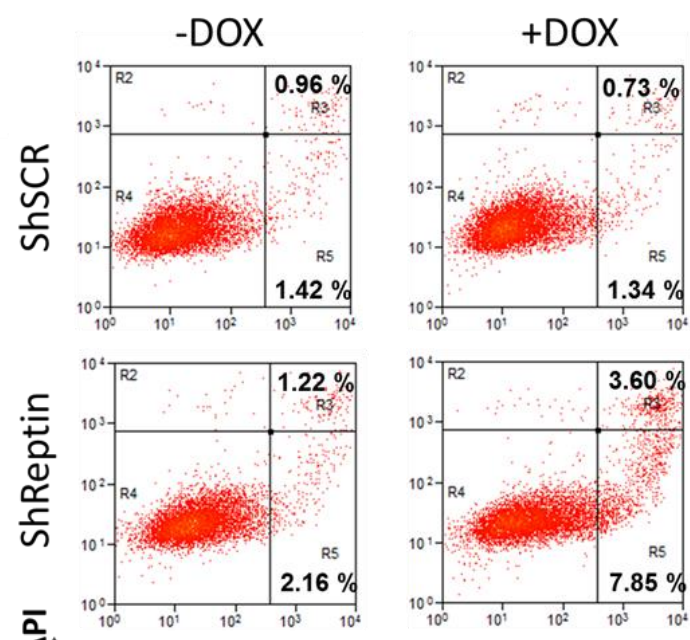

$\overline{\bar{a}_{\uparrow}}$

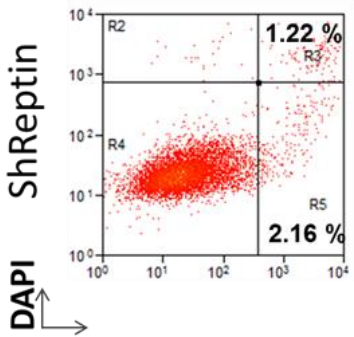

Annexin-V
B)

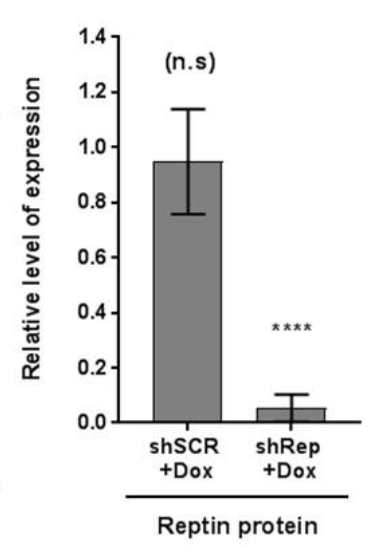

D)

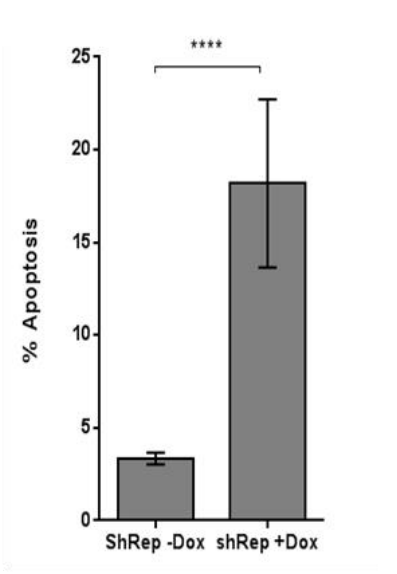

Figure 29. Characterisation of the experiments used for RNA-sequencing at time point Day 4.

(A) Western blot analysis of Reptin protein in DOX inducible scrambled shRNA (shSCR) and Reptin shRNA (shRep) transduced THP-1 cells following treatment with or without DOX for 4 days. Clathrin was used as a loading control. (B) Reptin protein levels relative to non-DOX (-DOX) treated control cells normalised to 1 , as quantified by the Quantity One software and normalised for the loading control. Columns represent the mean of five independent experiments and error bars the SD. $P$-values comparing normalised - DOX to + DOX samples were calculated using the 
one-sample $t$-test, $(* * * *) \quad p \leq 0.0001 ; \quad$ (n.s.) not significant $(p \geq 0.05)$. (C) Representative plot showing apoptosis levels in transduced cells from (A) at day 8 after DOX induction. For clarity purposes, these results have been also shown on figure 22. (D) Bar chart showing quantification of apoptosis levels of Reptin shRNA (shRep) transduced THP-1 cells following treatment with or without DOX for 8 days. Columns represent the mean of five independent experiments and error bars the SD. $P$-value was calculated using unpaired Student's $t$-test, $(* * * *) p \leq 0.0001$. 


\subsubsection{Global gene expression analysis following Reptin silencing}

RNA-Sequencing is a powerful method that uses deep-sequencing technology to detect and analyse the transcriptome profile of cells (Wang et al., 2009). As described on subsection 4.2.1, we used this technology to generate gene expression profiles of THP-1 cells after Reptin depletion from the RNA of 3 different experiments at day 2 after DOX induction and of 5 different experiments at day 4 after DOX induction. In order to identify significant changes in gene expression among samples, the resulting sequencing data were examined using the RNA express workflow of the Illumina BaseSpace website. This workflow aligns the RNA-seq reads to the human genome using the STAR aligner, which then assigns the aligned reads to genes. Then, the differential gene expression analysis was performed using the DESeq2 software package (Figure 30).

Only 360 significant gene expression changes were found at day 2 following DOX treatment (Figure 31A), of which only 31 genes were changed 2-fold or above in DOX-treated versus not-treated cells (Figure 31B). Since Reptin is not a classical transcription factor and it modifies gene transcription by modulating the structure of the chromatin, it is possible that its loss may take longer to result in gene expression changes. Indeed, 4 days after DOX induction 3,830 significant gene changes were found (Figure 31C), of which 151 were 2-fold or above (Figure 31D).

To validate our sequencing results, we first confirmed changes in gene expression of previously described Reptin target genes. One of them is the metastasis 
suppressor gene $C D 82$ (also known as $K A I 1$ ), which was found to be repressed by a complex formed by Reptin, $\beta$-catenin and NF- $\kappa \mathrm{B}$ together with histone deacetylases in metastatic prostate cancer cells (Kim et al., 2005). Interestingly, this was found to be a very specific target of Reptin, since the same publication described Pontin as a transcriptional activator, instead of a repressor, of $C D 82$ expression in a complex with Tip60 and NF- $\kappa$ B. Consistent with the described function of Reptin as a repressor of CD82, our RNA-seq data indicated a 1.44-fold increase of CD82 transcript levels after 2 days (Figure 32 A) and a 2.46-fold change after 4 days (Figure $32 \mathrm{~B}$ ) of Reptin depletion. We then validated this finding by qRT-PCR. Even though we obtained a significant 1.5 -fold increase of $C D 82$ expression after DOX treatment in shSCR transduced cells, the fold increase was much higher after Reptin depletion (3 fold increase) (Figure 32C), validating the gene expression data.

A second transcriptional target described for Reptin is the telomerase reverse transcriptase (hTERT) gene, which we also used to validate our gene expression results. Reptin was found to bind the hTERT promoter and to activate its expression in co-operation with c-MYC. Moreover, a Reptin knockdown in gastric cancer cells led to a decrease in hTERT transcript levels (Li et al., 2010). Congruent with the published data, our RNA-seq results also showed a 1.77-fold and 2.65-fold decrease of the hTERT transcript at day 2 and day 4 of our gene expression results, respectively. We then validated the sequencing data, as we also found a significant decrease in $h T E R T$ transcript after Reptin knockdown by qRT-PCR (Figure 33). 
Once our results had been validated, we continued our analyses with the gene expression data at day 4, as the number of differentially expressed gene was considerably higher than at day 2 . Thus, we took the list of differentially expressed genes at day 4 after Reptin depletion and examined it using PANTHER software tools (Protein ANalysis THrough Evolutionary Relationships, http://pantherdb.org), which assemble genes with known relationships and identify relevant pathways in gene lists from large-scale genomic experiments (Mi et al., 2017). Most of the pathways identified were related to cell cycle, apoptosis and metabolic processes, probably due to the fact that cells start differentiating and dying after Reptin depletion (Figure 34) (Osaki et al., 2013). As we were interested in identifying the upstream transcriptional regulatory pathways regulated by Reptin, rather than the downstream cellular consequences, we decided not to focus on the pathways identified using PANTHER and try a different approach to analyse our data. 


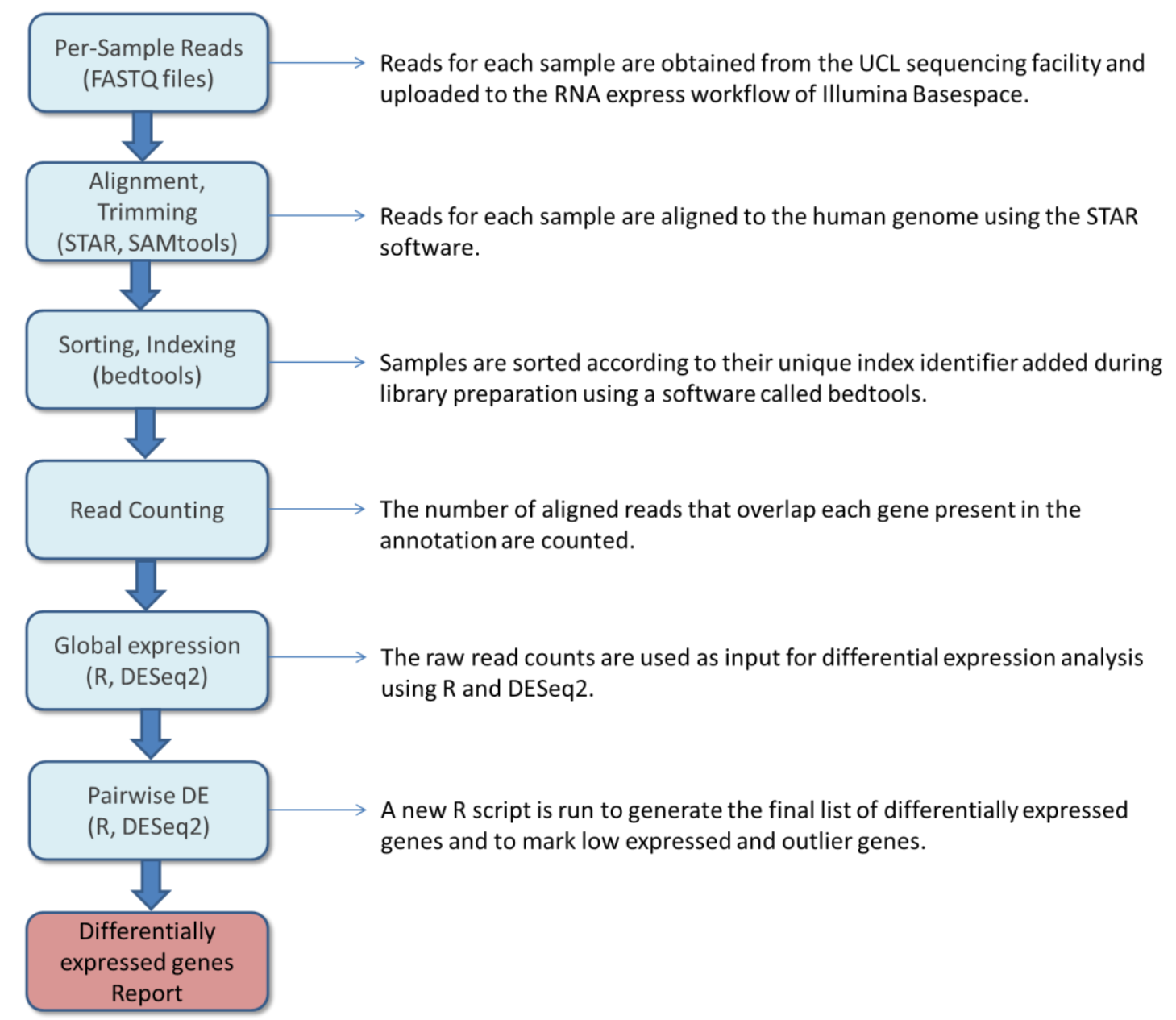

Figure 30. RNA express workflow.

Diagram depicting an overview of the different steps performed by the RNA express workflow used in this study to analyse RNA-sequencing data. Adapted from (Illumina, 2014). 
Day 2

A)

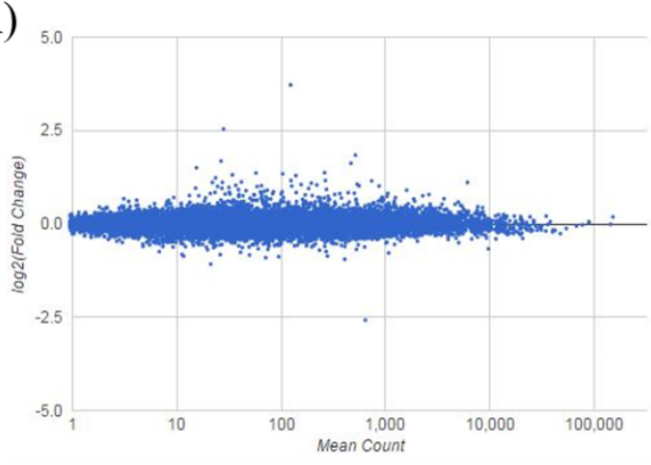

B)

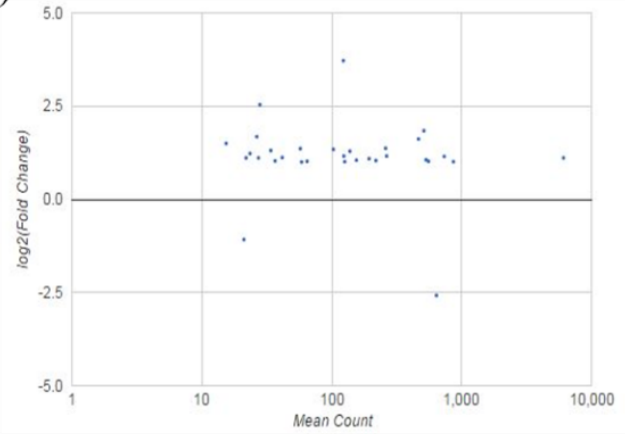

Day 4

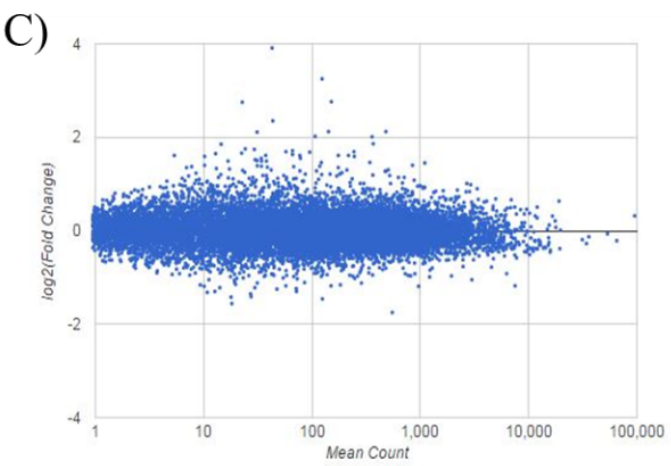

D)

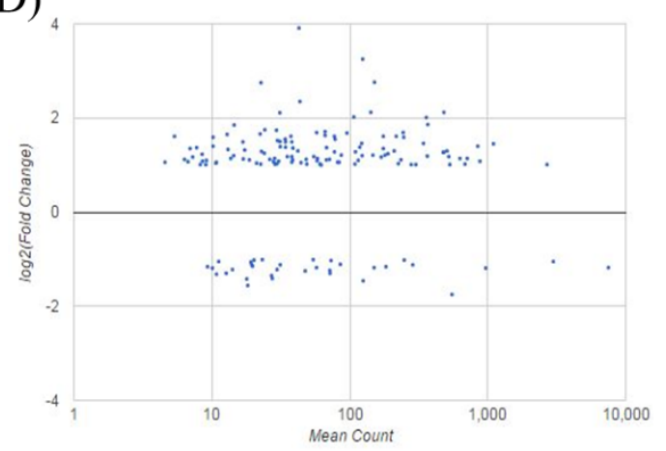

Figure 31. Analysis of differential gene expression after Reptin depletion.

Scatter plots generated by RNA express workflow on Illumina Basespace showing the total number of differentially expressed genes in shRep -DOX vs shRep +DOX at time point Day 2 (A) and Day 4 (C). The number of differentially expressed genes is reduced when a filter is applied to show genes with a fold change equal or higher than 2 at time point Day 2 (B) or Day 4 (D). The scatter plots display the $\log 2$ fold change against the mean count of a gene. Each dot represents a different gene. 
A)

Day 2

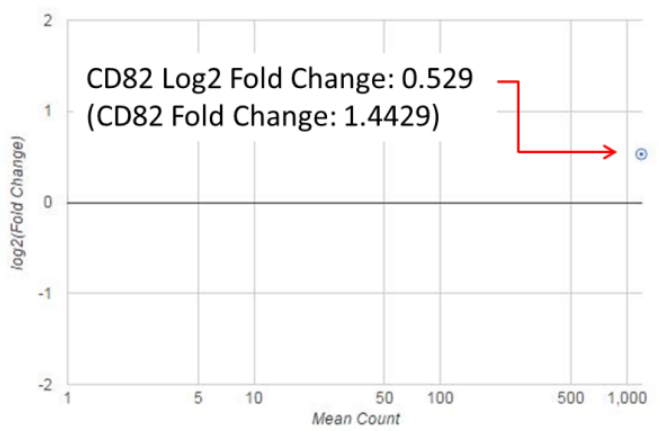

C)

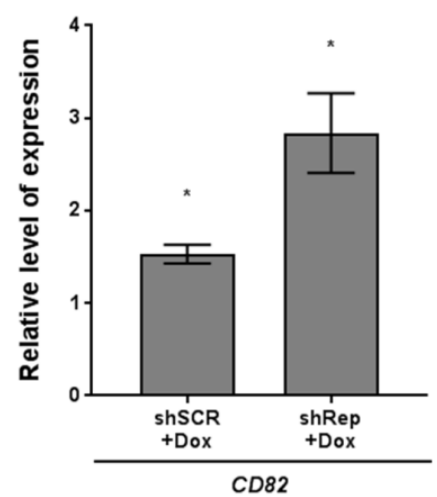

B)

Day 4

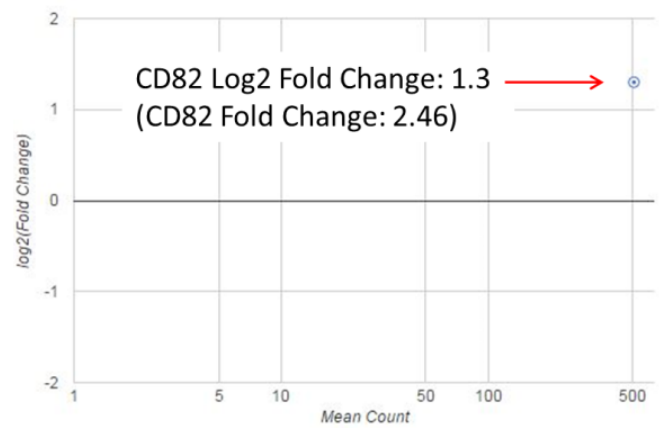

Figure 32. Reptin depletion leads to increased expression of CD82.

Scatter plots from Illumina Basespace showing the fold increase in CD82 levels following 2 days (A) or 4 days (B) of Reptin depletion. The scatter plots display the $\log 2$ fold change against the mean count of a gene. The circled dot represents the CD82 transcript. (C) Bar chart showing qRT-PCR analysis of CD82 mRNA expression levels in scrambled shRNA (shSCR) and Reptin shRNA (shRep) THP-1 cells induced with DOX for 4 days, relative to Non-DOX induced cells normalised to 1. Bars represent the mean of three independent experiments and error bars represent SD. $P$-values comparing normalised $-\mathrm{DOX}$ to $+\mathrm{DOX}$ samples were calculated using the one-sample $t$-test, $\left(^{*}\right) p \leq 0.05$. 
A)

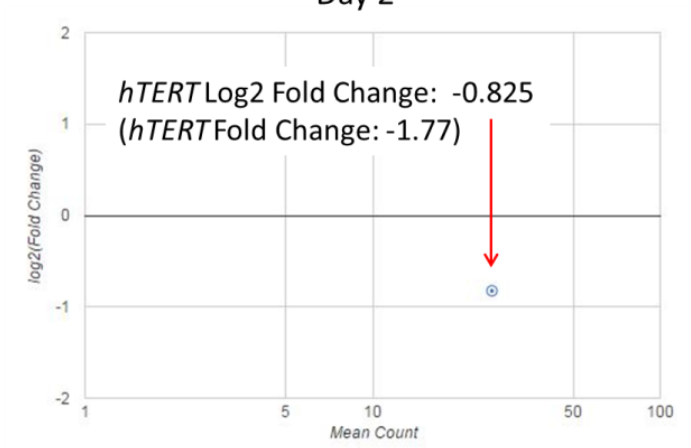

B)

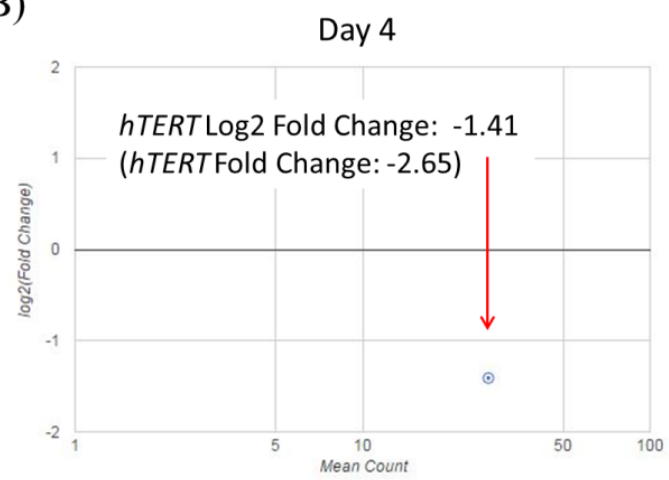

C)

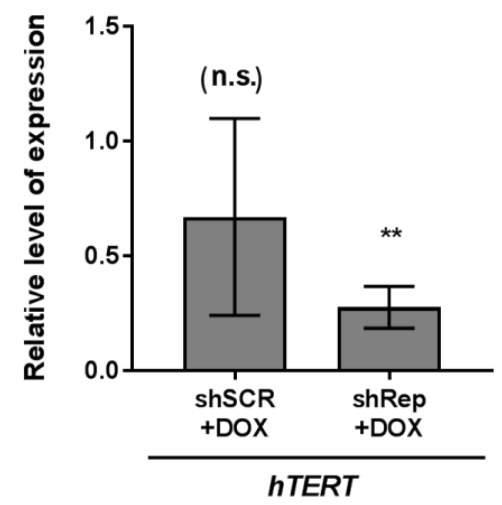

Figure 33. hTERT expression decreases after Reptin knockdown.

Scatter plots from Illumina Basespace showing the fold decrease in hTERT levels following 2 days (A) or 4 days (B) of inducible Reptin depletion. The scatter plots display the $\log 2$ fold change against the mean count of a gene. The circled dot represents the $h T E R T$ transcript. (C) Bar chart showing qRT-PCR analysis of $h T E R T$ mRNA expression levels in scrambled shRNA (shSCR) and Reptin shRNA (shRep) THP-1 cells induced with DOX for 4 days, relative to Non-DOX induced cells normalised to 1 . Bars represent the mean of three independent experiments and error bars represent SD. $P$-value comparing normalised $-\mathrm{DOX}$ to $+\mathrm{DOX}$ samples was calculated using the one-sample $t$-test, $(* *) p \leq 0.01$; (n.s.) not significant ( $p \geq 0.05$ ). 
A)

\begin{tabular}{|c|c|c|c|c|c|c|}
\hline PANTHER GO-Slim Biological Process & $\#$ & $\#$ & expected & Fold Enrichment & $+1=$ & $\underline{P \text { value }}$ \\
\hline IRNA metabolic process & 117 & $\underline{111}$ & 67.34 & 1.65 & + & $1.53 \mathrm{E}-04$ \\
\hline LRNA metabolic process & $\underline{2051}$ & 1522 & 1180.41 & 1.29 & + & 1.05E-21 \\
\hline 4nucleobase-containing compound metabolic process & $\underline{3160}$ & $\underline{2275}$ & 1818.67 & 1.25 & + & 3.05E-27 \\
\hline 4 primary metabolic process & $\underline{5773}$ & $\underline{3958}$ & 3322.53 & 1.19 & + & $6.20 \mathrm{E}-35$ \\
\hline 4 metabolic process & $\underline{6817}$ & $\underline{4640}$ & 3923.38 & 1.18 & + & $8.92 E-41$ \\
\hline tRNA metabolic process & 111 & 102 & 63.88 & 1.60 & + & $1.54 \mathrm{E}-03$ \\
\hline DNA repair & 172 & 153 & 98.99 & 1.55 & + & $6.30 \mathrm{E}-05$ \\
\hline 4DNA metabolic process & $\underline{373}$ & $\underline{288}$ & 214.67 & 1.34 & + & 2.10E-04 \\
\hline regulation of cell cycle & 109 & $\underline{96}$ & 62.73 & 1.53 & + & 1.32E-02 \\
\hline 4 cell cycle & $\underline{901}$ & $\underline{642}$ & 518.55 & 1.24 & + & 1.15E-05 \\
\hline 4 cellular process & $\underline{8199}$ & $\underline{4924}$ & 4718.76 & 1.04 & + & 1.67E-02 \\
\hline regulation of biological process & 1898 & $\underline{742}$ & 1092.35 & 68 & - & 1.24E-29 \\
\hline 4 biological regulation & $\underline{2288}$ & $\underline{943}$ & 1316.81 & .72 & - & 4.31E- -28 \\
\hline mRNA splicing, via spliceosome & 174 & 151 & 100.14 & 1.51 & + & 2.87E-04 \\
\hline 4 mRNA processing & $\underline{242}$ & $\underline{208}$ & 139.28 & 1.49 & + & $6.63 \mathrm{E}-06$ \\
\hline RNA splicing, via transesterification reactions & 134 & 115 & 77.12 & 1.49 & + & 7.64E-03 \\
\hline protein localization & $\underline{241}$ & $\underline{203}$ & 138.70 & 1.46 & + & 3.84E-05 \\
\hline protein complex assembly & 166 & 139 & 95.54 & 1.45 & + & 4.00E-03 \\
\hline 4 protein metabolic process & $\underline{2062}$ & 1390 & 1186.74 & 1.17 & + & 1.69E-07 \\
\hline 4 protein complex biogenesis & 167 & 140 & 96.11 & 1.46 & + & 3.55E-03 \\
\hline 4 cellular component biogenesis & $\underline{493}$ & $\underline{398}$ & 283.74 & 1.40 & + & 1.27E-08 \\
\hline
\end{tabular}

B)

\begin{tabular}{|c|c|c|c|c|c|}
\hline PANTHER Pathways & $\#$ & $\#$ & expected & Fold Enrichment & \pm Pvalue \\
\hline Ubiquitin proteasome pathway & $\underline{66}$ & $\underline{62}$ & 37.98 & 1.63 & $+3.29 \mathrm{E}-02$ \\
\hline Apoptosis signaling pathway & 122 & $\underline{107}$ & 70.21 & 1.52 & $+4.01 \mathrm{E}-03$ \\
\hline$\underline{\text { CCKR signaling map }}$ & $\underline{173}$ & $\underline{142}$ & 99.57 & 1.43 & $+5.36 \mathrm{E}-03$ \\
\hline Gonadotropin-releasing hormone receptor pathway & $\underline{235}$ & 182 & 135.25 & 1.35 & $+1.08 \mathrm{E}-02$ \\
\hline
\end{tabular}

\section{Figure 34. Gene Ontology and pathways analysis using PANTHER software.}

The figure shows the top Gene Ontology biological functions (A) and pathways (B) affected in THP-1 cells after Reptin depletion identified by PANTHER software tools (version 9.0). "\#” in the $3^{\text {rd }}$ column represents the number of genes in the uploaded list that map to the biological function or pathway. The "expected" value is the number of genes expected in the list for that category. The "Fold Enrichment" indicates an overrepresentation in the experiment if it is greater than 1 (represented with a "+" sign), or underrepresentation if it is lower than 1 (represented with a "-" sign). 


\subsubsection{Reptin modulates the LSC, c-MYC and c-MYB signatures}

We then analysed the sequencing results using the Gene Set Enrichment Analysis (GSEA) software. GSEA is a powerful computational method that evaluates gene expression data at the level of gene sets. Using statistical approaches, it interprets whether an a priori defined set of genes is positively or negatively enriched in a new list of differentially expressed genes, for example those occurring after Reptin loss (Subramanian et al., 2005). An advantage of this method over analysis on an individual gene level is that it can identify patterns of gene expression changes, even when the overall changes are modest. GSEA results are ordered according to the normalised enrichment score (NES). The NES represents the degree to which the gene set is overrepresented at the top or bottom of the ranked list of genes in the expression dataset, normalised across analysed gene sets. To ensure meaningful results from the GSEA analysis, the low expressed genes were removed from the list of genes uploaded to the software using the LOW filter option of the RNA express workflow.

Interestingly, GSEA analyses showed a negative correlation of the Leukaemic Stem Cell (LSC) signature (Somervaille et al., 2009) after Reptin depletion (Figure 35). This result suggests that Reptin expression is required to maintain leukaemic stem cells in AML, a population of self-renewing cells which sustain the disease. Thus, our GSEA result is in agreement with our previous data showing a reduced number of colonies in THP-1 cells transduced with a an shRNA targeting Reptin or the Dominant Negative mutant of Reptin (Osaki et al., 2013). 
Our GSEA analyses also showed a negative correlation of the c-MYC gene expression signature (Schuhmacher et al., 2001, Kim et al., 2006b) after Reptin depletion (Figure 36). This result was not surprising, since we had already seen a decrease in c-MYC protein and mRNA levels after Reptin depletion, and indicates that Reptin is necessary for proper activation and repression of $c-M Y C$ target genes. Although $c-M Y C$ RNA levels were not changed 2 days after of DOX induction, probably because $48 \mathrm{~h}$ of induction were not enough to detect many Reptin-induced gene expression changes, our sequencing results showed a reduction of $c-M Y C$ RNA levels after 4 days of Reptin depletion (Figure 37), confirming our previous findings using qRT-PCR. Overall, these data indicate that Reptin silencing results in the reduction of the $c-M Y C$ transcript and therefore, in a global alteration of its transcriptional signature.

$c-M Y C$ is a well stablished downstream target of the transcription factor cMYB in myeloid leukaemias (Schmidt et al., 2000). Moreover, $c-M Y B$ is a crucial downstream target of the MLL-fusions and a transcription factor playing a major role in sustaining the LSC population in $M L L$-rearranged leukaemia (Hess et al., 2006) (Somervaille et al., 2009). Thus, we hypothesised that the effects seen on $c-M Y C$, and also the phenotypic results observed after of Reptin loss, could be a consequence of Reptin being involved in the modulation of the upstream regulator c-MYB. We then generated a c-MYB gene expression signature. This list was obtained by integrating a list of genes whose promoters are bound by c-MYB in immortalized mouse myeloid cells (Zhao et al., 2011), as determined by Chromatin Immunoprecipitation (ChIP) sequencing (ChIP-seq) analysis, with genes 
differentially expressed in THP-1 cells following siRNA-mediated $c-M Y B$ silencing (Suzuki et al., 2009). The gene set is divided into genes activated and genes repressed by c-MYB. We then uploaded this list to the GSEA software and performed the analysis. Remarkably, our results indicated a highly significant negative correlation of the c-MYB signature in Reptin depleted cells (Figure 38). The lists of top 30 genes contributing the most to the enrichment in the activated and repressed GSEA analyses are shown in Table 13 . Notably, the $c-M Y B$ transcript was not found to be changed after Reptin depletion (Figure 39), confirming the absence of any change in c-MYB protein expression (Figure 18). 

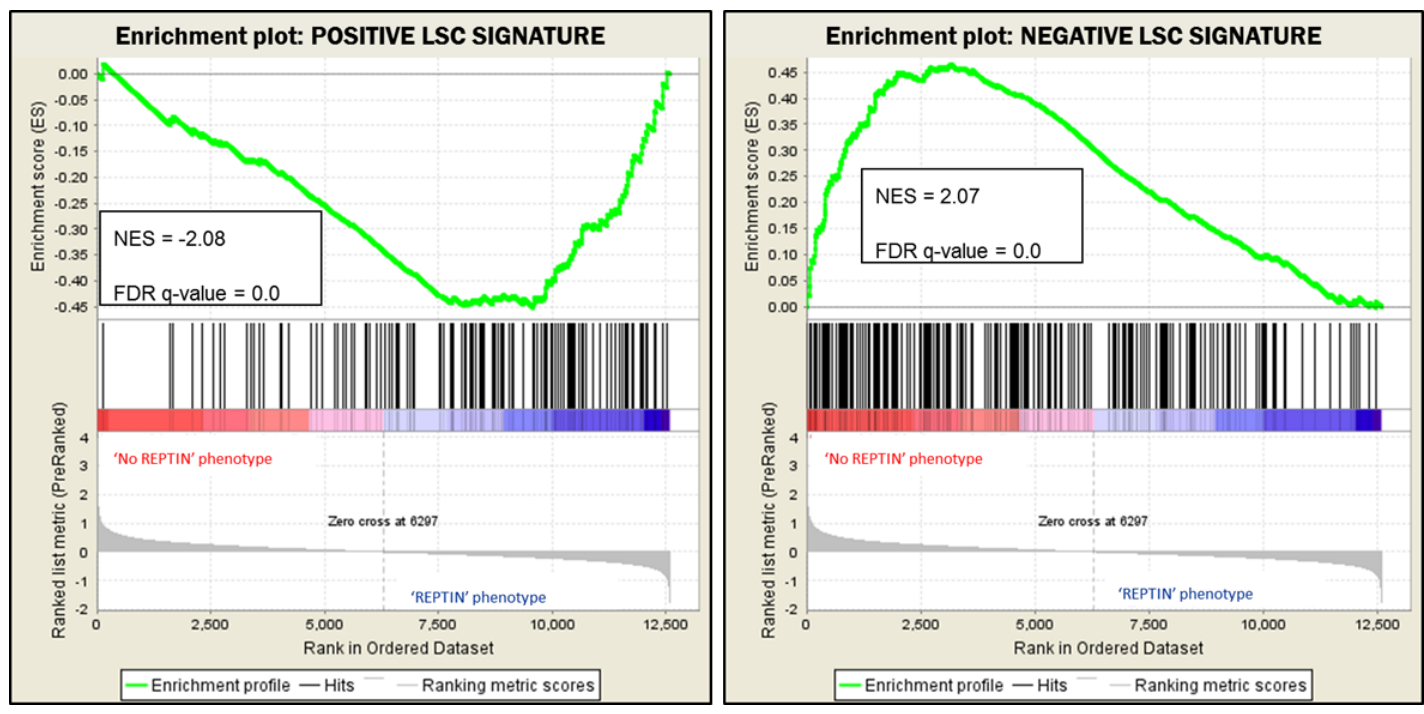

Figure 35. GSEA plots show a negative correlation of the LSC signature after Reptin depletion.

The figure shows GSEA enrichment plots of the positive (left) and negative (right) LSC signatures (Somervaille et al., 2009) for Reptin depleted (NO REPTIN) vs. Reptin expressing (REPTIN) cells at time point day 4. NES, normalised enrichment score; FDR, false discovery rate. 

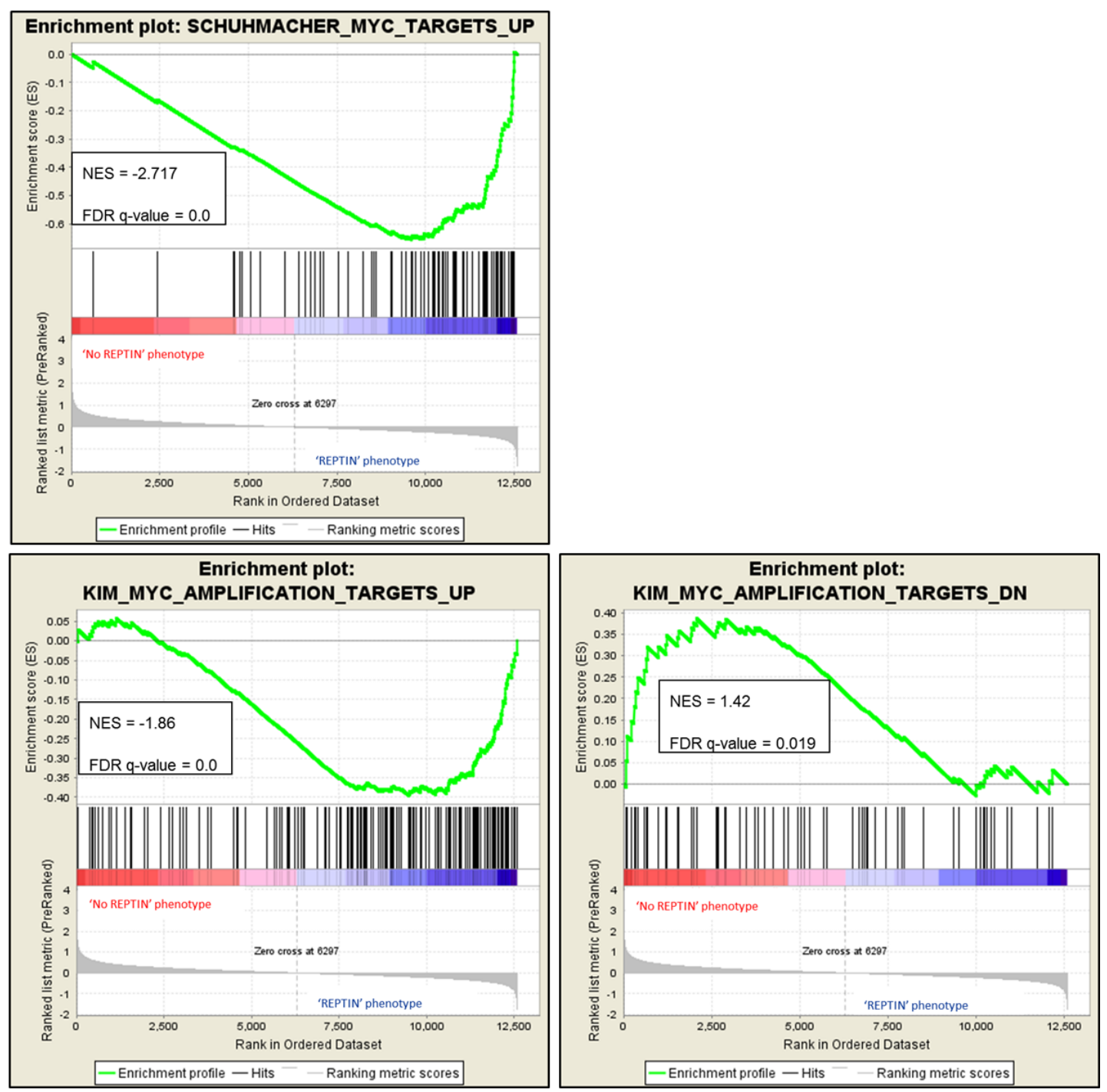

Figure 36. GSEA plots show a negative correlation of the c-MYC signature after Reptin depletion.

The figure shows GSEA enrichment plots of the c-MYC upregulated (UP) and downregulated (DN) gene sets (Schuhmacher et al., 2001) (Kim et al., 2006b) for Reptin depleted (NO REPTIN) vs. Reptin expressing (REPTIN) cells at time point day 4. NES, normalised enrichment score; FDR, false discovery rate. 


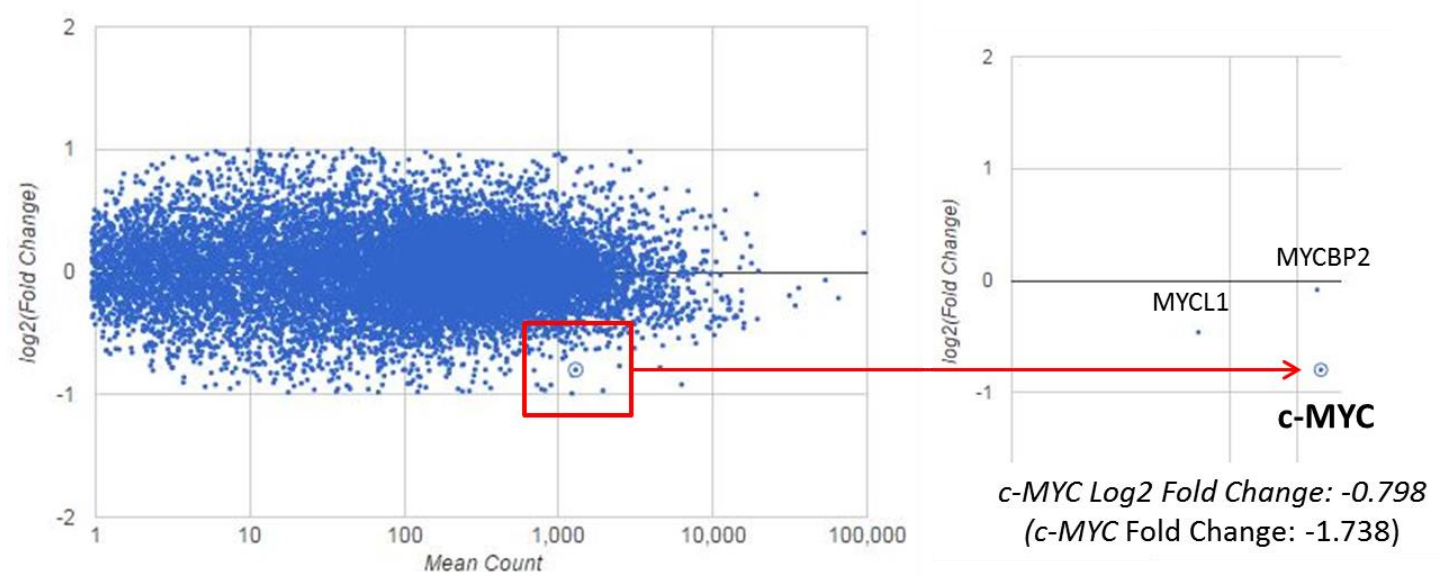

Figure 37. Sequencing results show a decrease in $c-M Y C$ expression levels after

\section{4 days of Reptin depletion.}

Scatter plot from Illumina Basespace showing the 1.738 fold decrease in $c-M Y C$ levels after Reptin depletion (time point Day4). The scatter plot displays the $\log 2$ fold change against the mean count of a gene. Each dot represents a different gene and the circled dot represents the $c-M Y C$ transcript. 

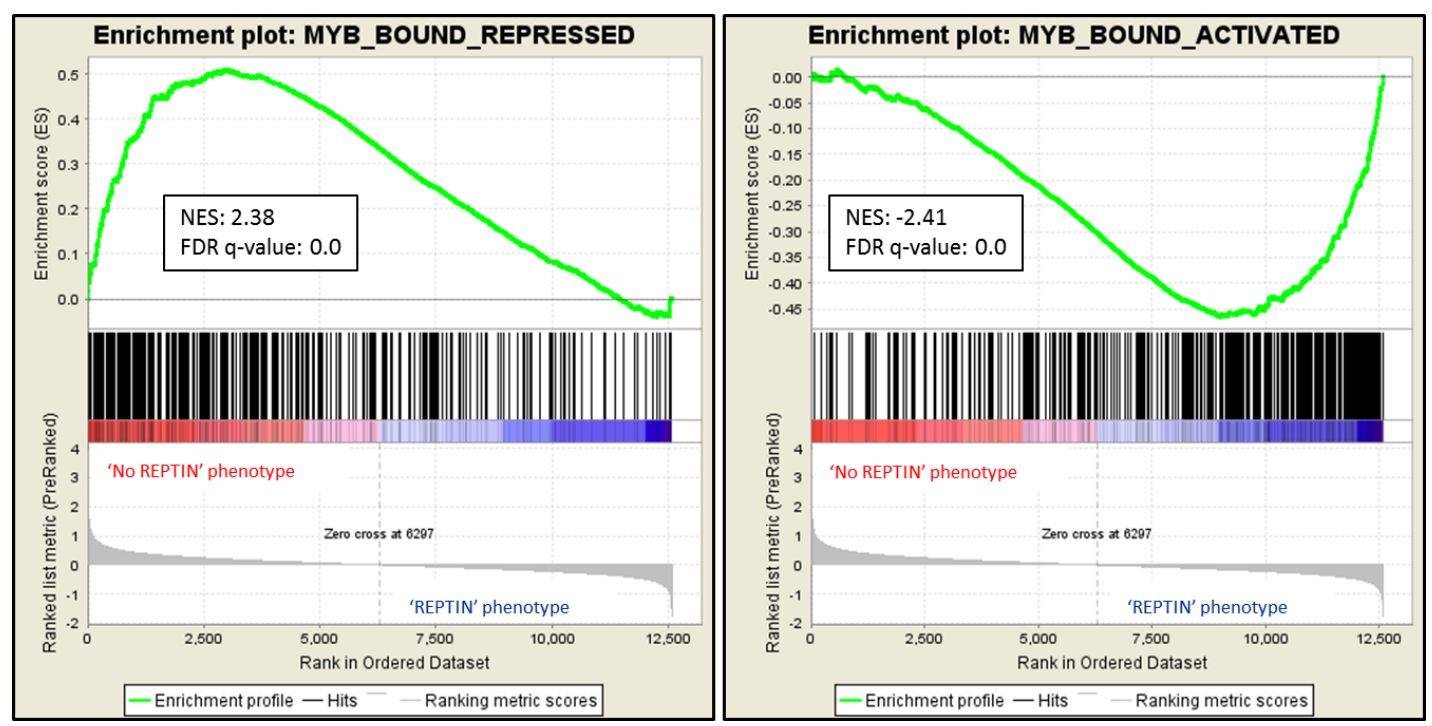

Figure 38. GSEA plots show a negative correlation of the c-MYB signature after Reptin loss.

The figure shows GSEA enrichment plots of the c-MYB repressed (left) and activated (right) gene sets (Zhao et al., 2011) for Reptin depleted (NO REPTIN) vs. Reptin expressing (REPTIN) cells at time point Day 4. NES, normalised enrichment score; FDR, false discovery rate. 


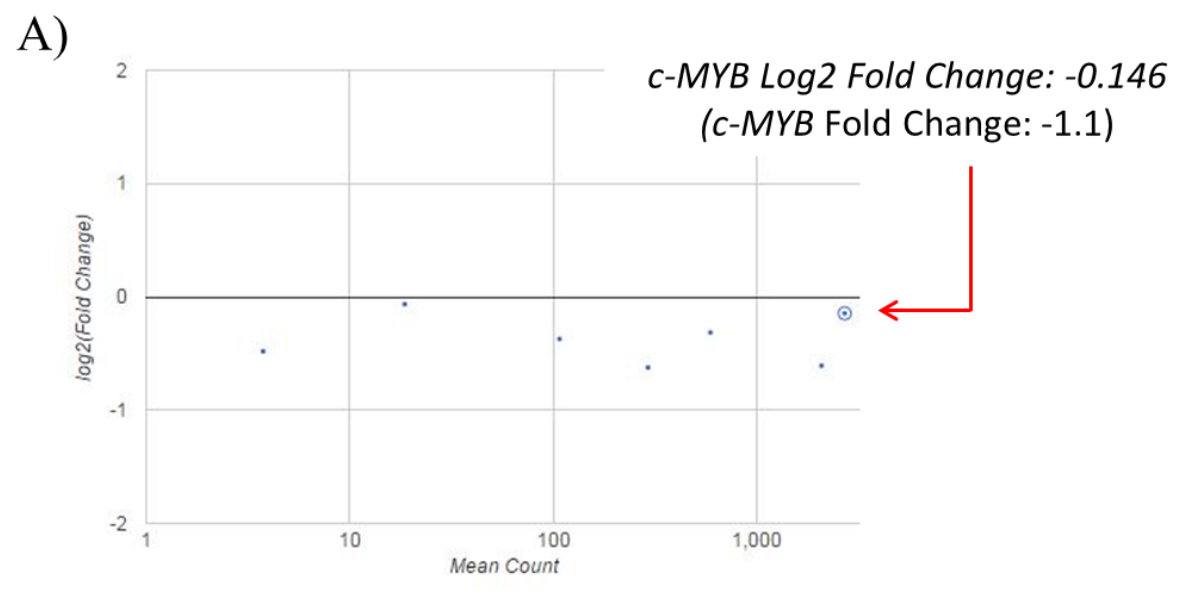

B)

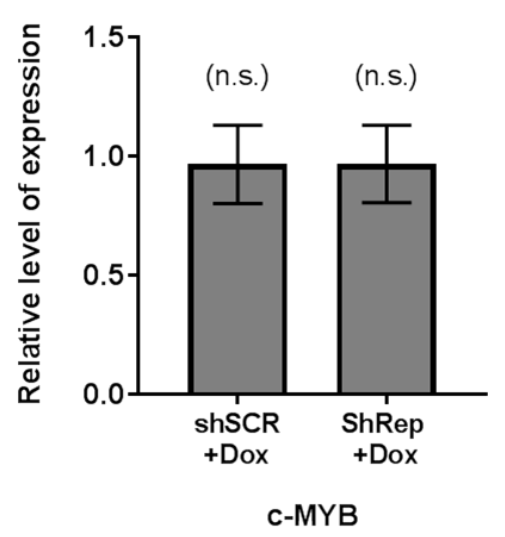

Figure 39. c-MYB transcript does not change after 4 days of Reptin depletion.

(A) Scatter plot from Illumina Basespace showing that c-MYB transcript levels don't change after Reptin depletion (Day4). The scatter plot displays the $\log 2$ fold change against the mean count of a gene. Each dot represents a different gene and the circled dot represents $c-M Y B$. (B) Bar chart showing qRT-PCR analysis of $c-M Y B$ transcript levels in DOX-induced scrambled shRNA (shSCR) and Reptin shRNA (shRep) THP-1 cells relative to Non-DOX induced cells. These data are representative of three independent experiments. $P$-values comparing normalised $-\mathrm{DOX}$ to $+\mathrm{DOX}$ samples were calculated using the one-sample $t$-test; (n.s.) not significant ( $p \geq 0.05)$. 


\begin{tabular}{|c|c|c|c|}
\hline \multicolumn{2}{|c|}{$\begin{array}{l}\text { GSEA c-MYB } \\
\text { REPRESSED }\end{array}$} & \multicolumn{2}{|c|}{$\begin{array}{l}\text { GSEA c-MYB } \\
\text { ACTIVATED }\end{array}$} \\
\hline $\begin{array}{c}\text { GENE } \\
\text { SYMBOL }\end{array}$ & $\begin{array}{c}\text { FOLD } \\
\text { CHANGE }\end{array}$ & $\begin{array}{c}\text { GENE } \\
\text { SYMBOL }\end{array}$ & $\begin{array}{c}\text { FOLD } \\
\text { CHANGE }\end{array}$ \\
\hline ANTXR1 & 4.347 & UGT3A2 & -2.462 \\
\hline DUSP4 & 4.056 & MS4A3 & -2.282 \\
\hline $\mathrm{KCNJ} 2$ & 2.809 & DHRS11 & -1.907 \\
\hline ATP8B 1 & 2.549 & GLDC & -1.826 \\
\hline SAMSN1 & 2.158 & FBXO43 & -1.800 \\
\hline IFI30 & 2.114 & SLC25A10 & -1.780 \\
\hline PALLD & 1.923 & MYC & -1.739 \\
\hline ANXA2 & 1.811 & NEIL3 & -1.736 \\
\hline CDKN1A & 1.808 & MPO & -1.714 \\
\hline DAPK2 & 1.803 & SEMA3A & -1.696 \\
\hline PRKCA & 1.780 & BNIP3 & -1.681 \\
\hline MAF & 1.769 & PLD6 & -1.671 \\
\hline DUSP6 & 1.768 & TAF9B & -1.629 \\
\hline SIGLEC5 & 1.725 & AKAP7 & -1.623 \\
\hline LPP & 1.717 & CXCR4 & -1.600 \\
\hline PHLDA1 & 1.711 & SERPINB2 & -1.582 \\
\hline FXYD5 & 1.709 & CDCA7L & -1.579 \\
\hline DAB2 & 1.681 & GPR160 & -1.550 \\
\hline NEU1 & 1.677 & SESN3 & -1.548 \\
\hline SLC27A3 & 1.659 & DEPDC1 & -1.526 \\
\hline VIM & 1.649 & CACNA2D3 & -1.521 \\
\hline CD9 & 1.629 & TMEM107 & -1.519 \\
\hline TNFRSF14 & 1.622 & CDKN3 & -1.516 \\
\hline FAM20C & 1.619 & IMPA2 & -1.508 \\
\hline LMNA & 1.601 & TRIM45 & -1.506 \\
\hline PTGS1 & 1.594 & ACOT7 & -1.487 \\
\hline SLC9A1 & 1.588 & CCDC34 & -1.486 \\
\hline ADORA3 & 1.560 & P2RY2 & -1.485 \\
\hline HCK & 1.546 & C4orf46 & -1.485 \\
\hline S100A10 & 1.538 & FBXO4 & -1.481 \\
\hline
\end{tabular}

Table 13. Top 30 enriched genes for the $c-M Y B$ activated GSEA signature.

The table shows the 30 most enriched genes for the $c-M Y B$ Repressed and Activated expression dataset in THP- 1 cells 4 days after Reptin depletion. The fold change after 
Reptin depletion is indicated next to the gene symbol. A negative value indicates a decrease in gene expression, while a positive value indicates an increase in gene expression. 


\subsection{Discussion}

Reptin had been previously described by our group as a critical mediator of MLLdriven oncogenesis in AML (Osaki et al., 2013). Moreover, in chapter III of this study we have also described a dependency on Reptin expression for leukaemia progression in vivo. In this chapter we have investigated the molecular mechanisms that render AML cells dependent on Reptin expression for survival, and describe the methods we employed to generate global transcriptome analysis after Reptin depletion, as well as the resulting data. Our results indicate that Reptin modulates cMYC protein stability and transcript levels, and identify the LSC, c-MYC and cMYB genetic signatures as being regulated by Reptin in AML.

\subsubsection{Reptin regulates c-MYC protein and transcript levels}

In order to elucidate the possible mechanism of action of Reptin in AML we initially studied the role of Reptin on c-MYC function, as Reptin was found to interact with c-MYC in a human cancer cell line (Wood et al., 2000) and modulate its function in cells from different model organisms (Etard et al., 2005) (Si et al., 2010). Moreover, previous work in our laboratory detected a marked decrease in c-MYC protein in a number of different AML cell lines after Reptin knockdown $(\mathrm{PhD}$ thesis, Zhao, 2013). Our data presented here are consistent with previous results obtained in our group, as we observed around a 50\% reduction in c-MYC protein after 4 days of Reptin knockdown. In addition, as Reptin and Pontin were found to enhance the cMYC/MIZ-1 repression of p21 in Xenopus Laevis (Etard et al., 2005), we also performed western blot analysis of the c-MYC partner protein MIZ-1 and observed a 
decrease of MIZ-1 after Reptin depletion, suggesting that probably it is not just cMYC but the whole repressive complex formed by c-MYC and MIZ-1 that is degraded when Reptin is not present in the cells.

We extended our analysis to examine the effect on c-MYC of an ATPasedeficient mutant of Reptin, which had previously been described to have the same deleterious consequences on proliferation as Reptin depletion (Osaki et al., 2013). Interestingly, the effect on c-MYC protein degradation was even stronger than the one obtained with the knockdown, as we observed a $78 \%$ reduction in c-MYC after 4 days of mutant expression, indicating that the ATPase domain of Reptin is necessary for its function on c-MYC. This finding was not surprising, since the ATPase activity of Pontin was found to be essential for c-MYC-mediated oncogenic transformation of rat embryo fibroblasts transduced with the oncogene H-rasG12V (Wood et al., 2000). Moreover, a dominant negative mutant of Pontin was found to promote cMYC mediated apoptosis (Dugan et al., 2002) and to be necessary for $\beta$-catenin driven oncogenesis (Feng et al., 2003). Even though these results were obtained using Pontin instead of Reptin mutants, they are still indicative that the ATPase activity of Pontin and Reptin are essential for some of their functions within the cell, and to the c-MYC pathway in particular.

We also studied the effect of subunit depletion for some of the described Reptin-containing complexes with a role in modulating c-MYC activity. MTBP is an oncogenic protein that was found to be overexpressed in different human cancers and to promote c-MYC driven transformation in conjunction with Reptin and Pontin 
(Grieb et al., 2014). Moreover, mice expressing reduced levels of MTBP or $c-M Y C$ were found to have a significant delay in spontaneous cancer development, particularly prominent in the haematopoietic compartment (Grieb et al., 2016). Consistent with our previously described findings, a knockdown of $M T B P$ also resulted in a decrease in c-MYC protein in the AML cell line THP-1. Although Reptin levels did not change after $M T B P$ depletion, we observed a decrease in MTBP protein after Reptin knockdown. As MTBP was recently found to modulate c-MYC oncogenicity through interaction with Reptin and Pontin (Grieb et al., 2014), and as Reptin has been found to have a chaperone-like function in many complexes (Nano and Houry, 2013), it is possible that AML cells depend on Reptin to maintain a functional interaction between MTBP and c-MYC to survive.

EP400 is a chromatin regulatory complex with transforming capacity, which includes both Reptin and Pontin but also EPC1 and EPC2 (Fuchs et al., 2001). In a previous study, depletion of the EP400 complex components EPC1, EPC2, Reptin and Pontin in THP-1 cells led to accumulation of c-MYC protein levels and an increase in cell death (Huang et al., 2014). We found these findings surprising, considering our data indicated a decrease in c-MYC after Reptin or MTBP depletion. We decided to replicate the experiments from Huang et al., and knocked down EPC1 and EPC2 in our THP-1 cells. In contrast to the published results, we observed a decrease in c-MYC protein instead of an increase. Importantly, we had verified our THP-1 cells by using the Short Tandem Repeat DNA profiling (STR profiling). Even though this finding was in agreement with our previous results, it is difficult to reconcile with the data published by Huang et al. A possible explanation for this 
contradiction could be the different source of THP-1 cells, as we obtained our THP-1 cells from ATCC (Rockville, USA) and they obtained their cells from DSMZ (Braunschweig, Germany). However, regardless of the source of the cell line and the effect on c-MYC, both studies indicate a regulation of c-MYC by the EP400 complex components. Our results are also consistent with the phenotype they observed, as they also described an increase in apoptosis after EPC1/2 and Reptin/Pontin knockdowns. These effects were the same as those obtained following Reptin silencing. This suggests that these subunits may exist in one or more complexes together with Reptin, regulating the function of c-MYC.

Importantly, in addition to a decrease in c-MYC protein levels after Reptin knockdown, or knockdown of Reptin interacting proteins, we also observed a decrease in $c-M Y C$ transcript levels after Reptin depletion. In particular, we found an average of $50 \%$ reduction in $c-M Y C$ mRNA levels after 4 days of Reptin knockdown in a bulk population of THP-1 cells. This reduction was not observed in a previous study done by our group ( $\mathrm{PhD}$ thesis, Zhao, 2013). The possible reason for this discrepancy could be the lower levels of Reptin knockdown achieved in that study in comparison to this one (60\% Reptin knockdown vs 95\% Reptin knockdown after 5 and 4 days of DOX treatment, respectively). Moreover, the experiments performed in that study were done in THP-1 derived clones, rather than the bulk of THP-1 cells. Thus, it is possible that the difference observed between the two studied is due to the intrinsic characteristics of the clones used, which differ from the bulk of the cells. However, the $60 \%$ Reptin knockdown in that study was enough to observe reduced levels of c-MYC protein, even when no changes in transcript levels were found $(\mathrm{PhD}$ 
thesis, Zhao, 2013). In contrast, in this study it was not possible to detect the loss in c-MYC protein prior to a decrease in $c-M Y C$ transcript levels, as they were always found simultaneously. This suggested that the effects seen on c-MYC after Reptin depletion could be the consequence of the modulation by Reptin of an upstream transcriptional regulator of $c-M Y C$, which would lead to a decrease in $c-M Y C$ transcript and the subsequent protein loss through normal turnover.

Several previous studies have described an interaction of Reptin and Pontin with c-MYC. Wood et al. found that Reptin and Pontin interact with c-MYC in human embryonic kidney cells through the box II domain (MBII), which is essential for c-MYC mediated transformation. However, only Pontin was described to have an essential role for c-MYC mediated transformation (Wood et al., 2000). Etard et al. overexpressed mutants of Xenopus Reptin and Pontin and showed that the N-terminal domain of xReptin and xPontin is required for their interaction with c-MYC (Etard et al., 2005). Moreover, Bellosta et al. described an interaction of Reptin and Pontin with Drosophila melanogaster dMyc, although the interaction of Reptin with dMYC was much weaker (Bellosta et al., 2005). Therefore, it appears that Pontin was found to have a stronger interaction or a more relevant role in modulating c-MYC function than Reptin. In this study, by using immunoprecipitation assays of 3xHA-tagged Reptin we were not able to detect the interaction of Reptin with c-MYC. Considering that Reptin has been found to interact with and modulate the function of many other proteins (Gallant, 2007), it is possible that, in AML cells, Reptin may be modulating the function of a different transcription factor. This would be concordant with our previous observation that $c-M Y C$ transcript levels decrease at the same time as the 
protein, which indicated that Reptin could be modulating an upstream regulator. However, we cannot rule out the possibility that the use of different antibodies or technique might be the reason for not finding this interaction in AML cells.

Taken together, the findings described here suggest that Reptin modulates $c$ $M Y C$ transcription, resulting in reduced levels of protein expression.

\subsubsection{Negative enrichment of the LSC, c-MYC and c-MYB signatures after Reptin depletion}

In order to identify the transcriptional targets of Reptin in AML we generated global gene expression data after 2 and 4 days of Reptin depletion. To avoid obtaining clone-specific transcriptional results we decided to use the bulk population of THP-1 cells. To evaluate the feasibility of using a bulk population instead of a clonal population for transcriptome analysis, we characterised the response to inducible Reptin depletion of the bulk of cells. THP-1 cells were transduced with an inducible vector containing a shRNA targeting Reptin or a control scrambled shSCR $(\mathrm{PhD}$ thesis, Zhao, 2013), and the level of induction and Reptin knockdown were assayed by flow cytometric and western blot analysis. The results indicated high levels of induction, as more than $90 \%$ of transduced cells expressed tRFP after just $24 \mathrm{~h}$ of DOX treatment. Moreover, a 5-fold increase in apoptosis was detected in DOX treated cells after 8 days in culture in comparison to untreated -DOX cells. As Reptin protein levels had also decreased by more than $70 \%$ after 2 days of DOX induction and by more than $90 \%$ after 4 days of DOX induction, the RNA of different replicate 
experiments at time points day 2 and 4 was extracted and used to generate a gene expression profile of the transduced cells by RNA-sequencing (RNA-seq).

RNA-seq is a deep-sequencing based technology that has been extensively used in recent years for transcriptome profiling because it offers several advantages over preceding technologies, including very low background signal noise and increased sensitivity for low-expressed genes (Wang et al., 2009). The validity of the results obtained using the RNA Express workflow of Illumina BaseSpace was initially assessed by analysing the already described transcriptional targets of Reptin CD82 and hTERT (Kim et al., 2005) (Li et al., 2010). Indeed, a significant change in both genes was detected in the analysis, as shown by the Illumina BaseSpace software. We then further validated these results by qRT-PCR. Although significant change in CD82 levels was detected after inducing shSCR transduced cells with DOX, the fold change in Reptin depleted cells was much greater (1.5 fold versus a 3 fold increase), indicating that Reptin does regulate CD82 gene expression in AML cells. Moreover, a significant decrease in hTERT was also detected by qRT-PCR analysis after Reptin depletion. Importantly, we were able to detect these changes even when the starting Reptin protein levels in shReptin -DOX cells were lower than in control shSCR cells due to leakiness in the system. Together, these findings validated our global gene expression results.

Analysis of the gene expression data using the pathways analysis tool PANTHER (http://pantherdb.org) only indicated a change in cell cycle, apoptosis and metabolic processes. As we had previously described an increase in 
differentiation and cell death in AML cells after Reptin depletion (Osaki et al., 2013), in this study we were more interested in finding the upstream pathways leading to these effects. Thus, we decided to use Gene Set Enrichment Analysis (GSEA) in order to analyse the data further. Interestingly, the GSEA results showed a negative enrichment of an MLL-rearranged AML Leukaemic Stem Cell signature (Somervaille et al., 2009). Leukaemic Stem Cells are normally a rare population of self-renewing cells that initiate and maintain the disease and, therefore, have been proposed as good targets for therapy (Bonnet, 2005) (Somervaille and Cleary, 2006). It is then relevant to understand which molecular mechanisms sustain this population in AML, in order to develop novel and more specific treatments. Our finding suggests that Reptin is necessary for the survival of LSC, confirming previous results from our group in which Reptin depleted AML cells failed to form colonies in colony forming assays (Osaki et al., 2013). As the LSC population was found to enrich for c-MYC target genes (Somervaille et al., 2009), and considering that both c-MYC protein and transcripts were found to be decreased in Reptin depleted cells, the effect seen here on the LSC signature could be the result of the defective c-MYC function, described in this chapter, after Reptin depletion. Whatever its cause, this result indicates that Reptin represents a potential target for therapy, since drugs targeting Reptin would be expected to impact on the LSC compartment in AMLs.

As both protein and transcript levels of $c-M Y C$ were found to be decreased after Reptin depletion, we anticipated that the c-MYC genetic signature would also be changed. Indeed, our GSEA analyses showed aberrant transcriptional activation and repression of c-MYC target genes upon Reptin knockdown. This is a relevant 
finding, since c-MYC has been found to be amplified or overexpressed in myeloid leukaemias and to inhibit myeloid cell differentiation (Delgado et al., 2013). However, in contrast to a previous study in our laboratory (PhD thesis, Zhao, 2013), in this study we were not able to detect a decrease in c-MYC protein levels before mRNA levels were affected, as we detected a simultaneous change in c-MYC protein and transcript levels. Thus, we hypothesised that the transcription factor c-MYB could be the leading cause for this effect, as it has previously been described as an upstream modulator of $c-M Y C$ in myeloid leukaemias (Schmidt et al., 2000). Strikingly, our GSEA analysis revealed a strong negative correlation of the c-MYB signature after Reptin depletion. In contrast to our observation with the $c-M Y C$ oncogene, $c-M Y B$ mRNA and protein levels were not decreased after depleting Reptin in THP-1 cells. This suggested that possibly the function of Reptin in modulating gene transcription as part of various chromatin remodelling complexes (Jha and Dutta, 2009) could be involved in regulating the expression of c-MYB target genes. Since c-MYB has an essential role in maintaining myeloid leukaemias (Pattabiraman and Gonda, 2013) and it has been a difficult target for therapy (Ramsay and Gonda, 2008), the finding that Reptin can modulate its genetic signature is significant, as one could envisage Reptin inhibition as an alternative strategy to direct c-MYB targeting.

In summary, by using global gene expression analysis, in this chapter we have identified the transcriptional pathways regulated by Reptin in AML. Our data suggest that the genetic signatures of essential transcription factors for leukaemia 
maintenance and progression, such as c-MYC and c-MYB, are dependent on Reptin expression. 


\section{CHAPTER V - Investigating the role of Reptin on c- MYB function}

\subsection{Introduction}

Our gene expression analyses, discussed in the previous chapter of this thesis, revealed a negative correlation of the c-MYB signature after Reptin depletion in AML cells. As mentioned in the introduction, c-MYB is a transcription factor with an essential role in both normal and aberrant haematopoiesis (Pattabiraman and Gonda, 2013). Even though Reptin has been found to modulate the function of different transcription factors with a role in cancer, including c-MYC (Wood et al., 2000) and E2F1 (Tarangelo et al., 2015), to our knowledge the effect of Reptin on the transcription factor c-MYB has not previously been described.

In this chapter we explored the potential regulatory role of Reptin on c-MYB function in AML. To that aim, we first validated our gene expression analyses by qRT-PCR in THP-1 cells transduced with the inducible system to deplete Reptin expression. In order to further validate our sequencing results, we generated additional global gene expression data after 7 days of constitutive Reptin depletion and validated the new sequencing results. Moreover, by performing coimmunoprecipitation assays we also identified an interaction of overexpressed and endogenous Reptin protein with endogenous c-MYB. Finally, we optimised and performed Chromatin Immunoprecipitation (ChIP) assays of c-MYB and H3K27ac, 
an active transcription epigenetic mark, to assess the effect of Reptin depletion on cMYB regulated gene promoters. Our experiments identified a marked decrease of H3K27ac and c-MYB binding in the promoter of a c-MYB regulated gene after Reptin loss.

Collectively, the data presented in this chapter suggest that Reptin regulates c-MYB function in human AML by forming a complex at the regulatory regions of c-MYB target genes. Moreover, our results have provided a rationale for performing additional experiments, such as ChIP-sequencing, to assess the effect of Reptin on cMYB chromatin binding on a global scale. 


\subsection{Results}

\subsubsection{Validating the c-MYB negative enrichment after Reptin depletion}

We used qRT-PCR analyses to validate our GSEA results that indicated a negative correlation of the c-MYB signature after Reptin loss. We selected some of the significantly changed genes that contributed the most to the negative enrichment, including genes shown to be activated (AKAP7, PLD6, MPO) and repressed (DUSP4, SAMSN1) by c-MYB. To ensure that the selected genes were true c-MYB target genes in THP-1 cells, we constitutively knocked down $c-M Y B$ in bulk THP-1 cells with two different shRNA sequences (shMYB.17 and shMYB.53). As expected, we obtained a significant decrease in the expression of the activated genes and an increase in expression of the repressed genes following c-MYB knockdown (Figure 40), indicating that indeed the selected genes were true c-MYB targets in THP-1 cells. We then analysed the effect of Reptin loss on the expression of the selected cMYB target genes. After DOX induction to deplete Reptin expression, we observed a smaller but significant change in expression of the repressed (Figure 41A) and activated (Figure 41B) genes.

To validate that the results of the expression data obtained were not a secondary effect of doxycycline treatment, we decided to generate new global gene expression data using a non-inducible system to deplete Reptin expression. The pGIPZ system for stable constitutive down regulation of Reptin had already been used and optimized in a previous study in our laboratory (PhD thesis, Osaki, 2011). In that study, the vector had been modified to contain the spleen focus-forming virus (SFFV) promoter, which is more active in haematopoietic cells than the original 
cytomegalovirus (CMV) promoter contained in the pGIPZ lentiviral system (Sirven et al., 2001). We transduced THP-1 cells with the control scrambled shRNA (shSCR) or the shRNA targeting Reptin and selected them with puromycin for 3 consecutive days. 7 days after transduction, the average level of Reptin protein knockdown achieved in three independent experiments was greater than $80 \%$ (Figure 42), and the average Reptin transcript knockdown was greater than 95\% (Figure 43 A). We also checked the mRNA levels of the described Reptin downstream target gene CD82 and detected an average 3-fold increase in expression (Figure $43 \mathrm{~B}$ ), indicating that we were able to detect non-DOX induced changes after Reptin depletion.

As this time we used a constitutive method to deplete Reptin expression, it was important to determine that similar virus titres were achieved for the control and experimental shRNAs used. Similar titres would indicate that the gene expression data reflect the loss of the targeted gene, rather than the different levels of shRNA expressed in each group. To test this, we transduced each group of cells with equivalent diluting concentrations of virus supernatant. As cells transduced with the pGIPZ construct also express the Green Fluorescent Protein (GFP), 3 days after transduction cells from the two different groups were analysed by flow cytometry to detect GFP expression (Figure 44). After confirming that similar levels of titres were achieved for the two different groups, we submitted the mRNA of three independent experiments for sequencing. We then analysed the data using the RNA express workflow of Illumina BaseSpace, as described in the previous chapter. As seen in Figure 45, in this new RNA-seq we obtained 583 upregulated and 1510 
downregulated genes by more than 1.5 fold after Reptin loss, of which 96 and 106, respectively, were in common with the RNA-seq performed at Day 4 with the inducible system.

To validate the sequencing data resulting from Illumina BaseSpace, we examined the fold-change in expression of the already described Reptin targets CD82 and $h T E R T$. As expected, we observed a 2.65 -fold decrease in $h T E R T$ and a 2.37 -fold increase in CD82 transcript levels (Figure 46), indicating a correct analysis of the sequencing data by the Illumina BaseSpace software. Importantly, we also confirmed that $c-M Y B$ transcript levels did not change in this experiment (Figure 47). We then performed GSEA analyses of the sequenced data, which confirmed the negative enrichment of the c-MYB repressed genes upon Reptin depletion (Figure 48). Surprisingly, we did not observe the same negative correlation with the c-MYB activated genes this time (Figure 48). We reasoned that this result could be attributed to the increased background noise existing due to the gene expression effects driven by the virus transduction and puromycin selection of the cells. Nonetheless, by qRTPCR we were able to validate genes from both repressed and activated c-MYB signatures (Figure 49).

The shRNA sequence embedded in both the pTRIPZ and pGIPZ systems used in this study is the same. A previous study in our laboratory already validated the deleterious effects of Reptin depletion with a different shRNA sequence (Osaki et $a l ., 2013)$. To validate that the effects seen on the c-MYB signature are not the result of an off-target effect, we used an alternative method of Reptin depletion. THP-1 
cells were transduced with two different MISSION pLKO.1 shRNA vectors containing different shRNA sequences (\#63 and \#66) and the mRNA levels of different c-MYB target genes were determined by qRT-PCR. We were able to successfully validate the c-MYB activated target genes with both shRNA sequences (\#63 and \#66) and the c-MYB repressed genes with one of them (\#63). Thus, these results indicate that the effects of Reptin depletion on the c-MYB signature are gene specific (Figure 50).

As mentioned in the introduction, in some cases Reptin and Pontin have been found to work independently from each other (Gallant, 2007). In order to assess whether Reptin and Pontin have an independent role in regulating c-MYB function, we depleted Pontin expression in THP-1 cells and performed qRT-PCR analysis of c-MYB target genes. As seen in Figure 51, our results did not show a significant downregulation of the activated c-MYB target genes, which suggested that Reptin and Pontin might not be functionally analogous in this activity. 

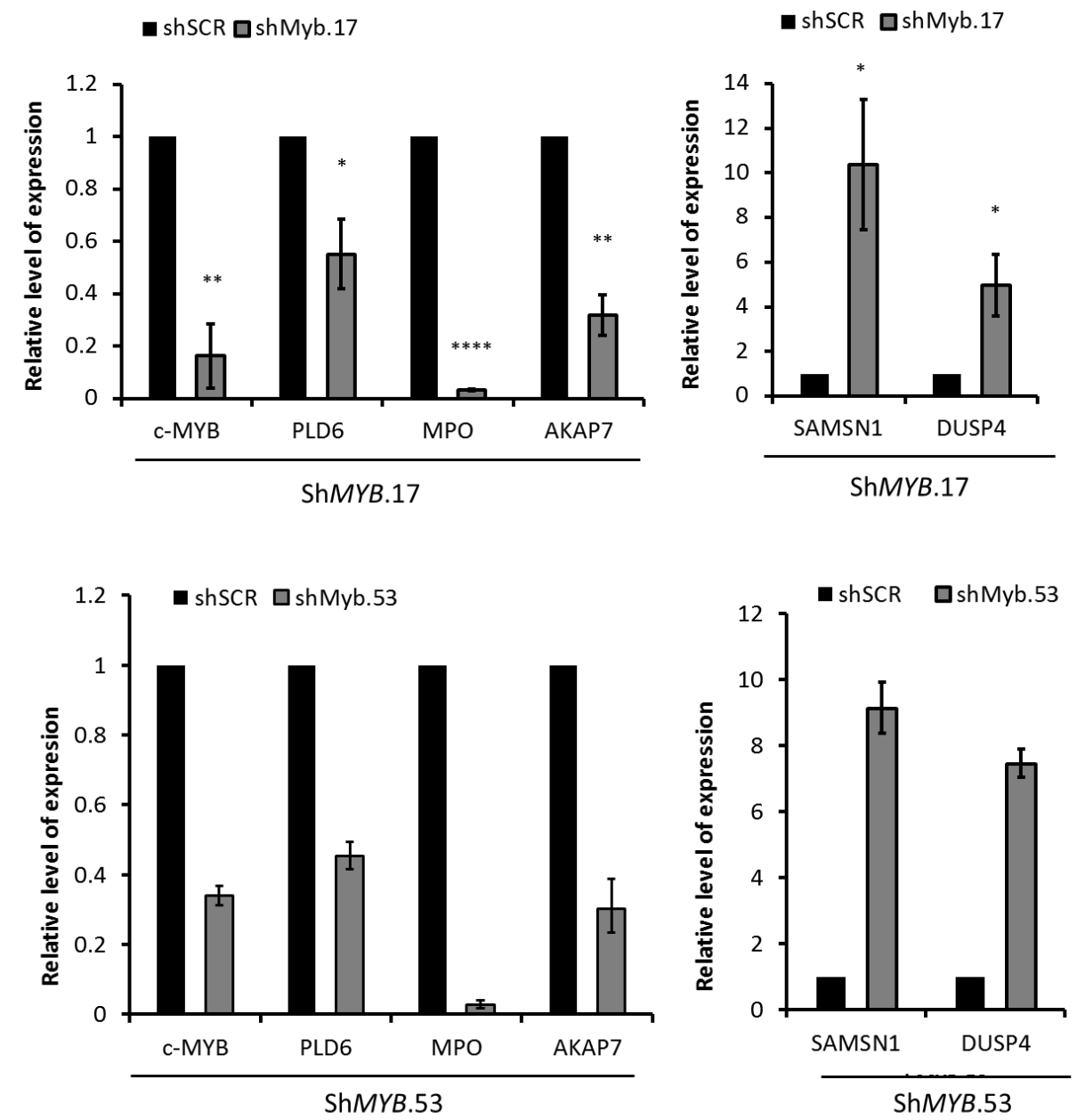

Figure 40. Validation of $c-M Y B$ target genes after $c-M Y B$ loss.

qRT-PCR analysis of c-MYB repressed or activated target genes in THP-1 cells depleted of c-MYB expression with 2 different shRNA (shMyb.17 and shMyb.53) relative to the expression in scrambled control (shSCR) cells normalised to 1. $c-M Y B$ mRNA levels are also indicated. For shMYB.17, columns represent the mean of three independent experiments and error bars represent SD. $P$-values were calculated using the one-sample $t$-test, $(* * * *) \quad p \leq 0.0001 ;(* *) p \leq 0.01 ;(*) p \leq 0.05$. For shMyb.53, columns represent the mean of triplicate measurements of a single experiment and error bars, the SD. 
A)

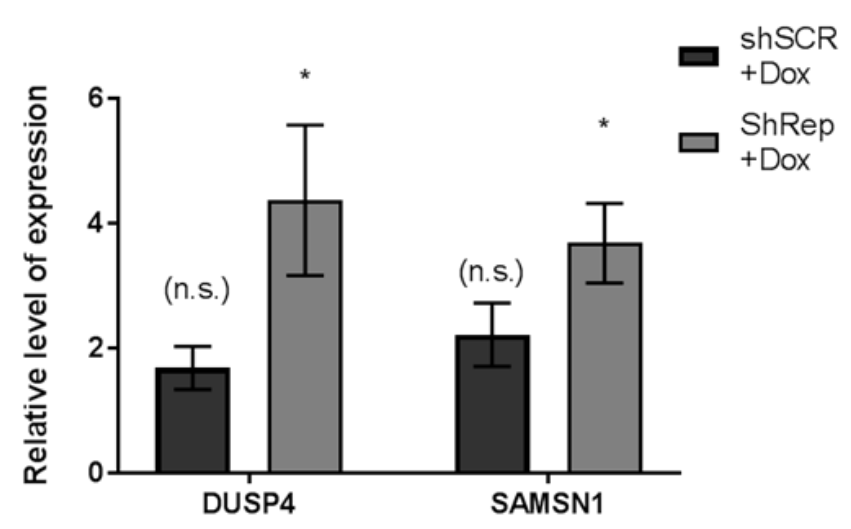

B)

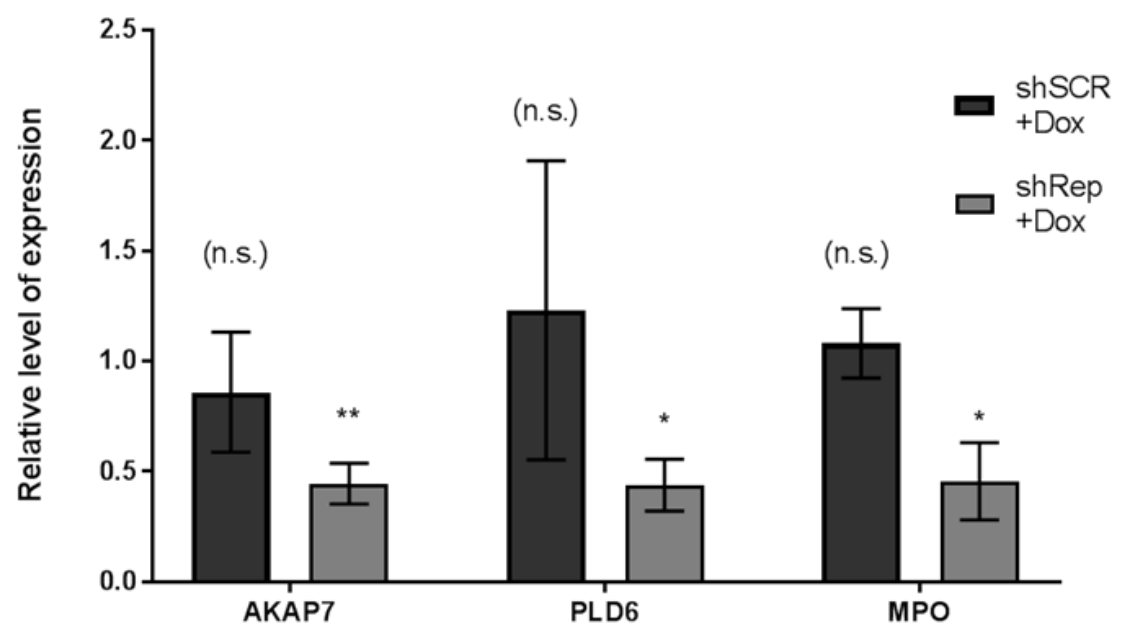

Figure 41. Validation of c-MYB target genes after inducible Reptin depletion.

qRT-PCR analysis of c-MYB repressed (A) or activated (B) target genes in DOXinduced scrambled shRNA (shSCR) and Reptin shRNA (shRep) THP-1 cells, relative to the expression in non-DOX induced cells normalised to 1 . Columns represent the mean of three independent experiments and error bars represent SD. $P$ values comparing normalised - DOX to + DOX samples were calculated using the one-sample $t$-test, $(* *) p \leq 0.01 ;\left(^{*}\right) p \leq 0.05$; (n.s.) not significant $(p \geq 0.05)$. 
A)

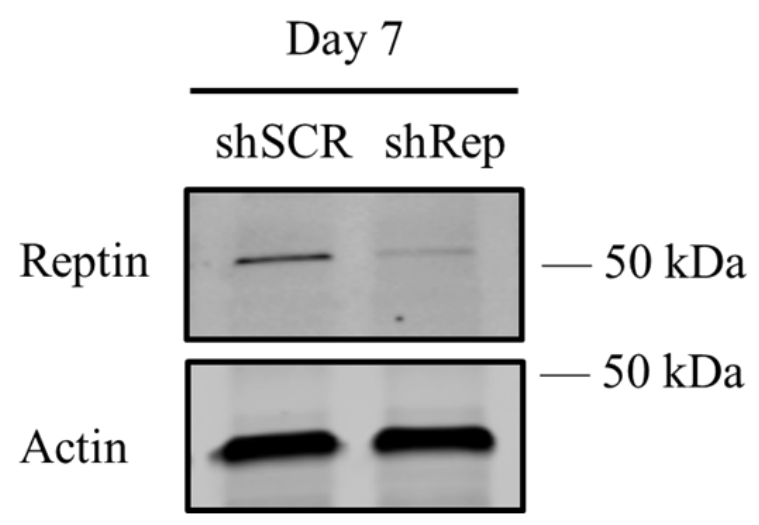

B)

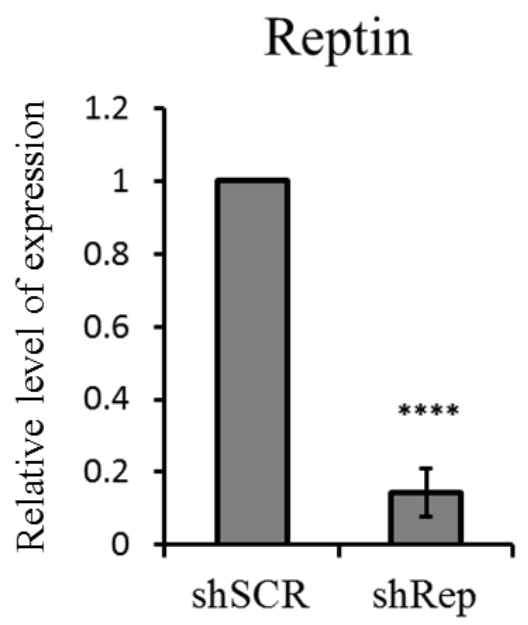

\section{Figure 42. Reptin protein levels after 7 days of constitutive Reptin depletion.}

(A) Representative western blot analysis of Reptin protein in constitutive scrambled shRNA (shSCR) and Reptin shRNA (shRep) transduced THP-1 cells after 7 days of transduction. Actin was used as a loading control. This experiment was performed three times. (B) The bar chart shows the densitometric quantification of Reptin protein levels after 7 days of constitutive Reptin depletion, relative to Actin. Data represents the mean of three independent experiments and the error bar represents the SD. shSCR data were normalised to $\mathrm{q}$ and a $p$-value comparing shRep to normalised shSCR was calculated using the one-sample $t$-test; $(* * * *) p \leq 0.0001$. 
A)

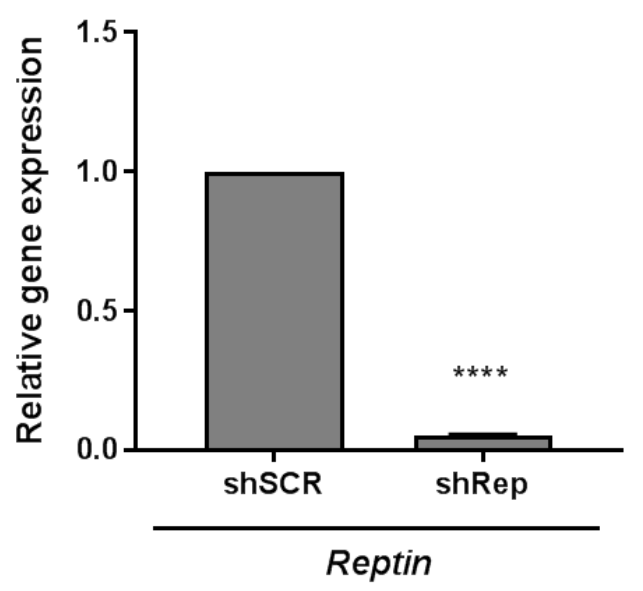

B)

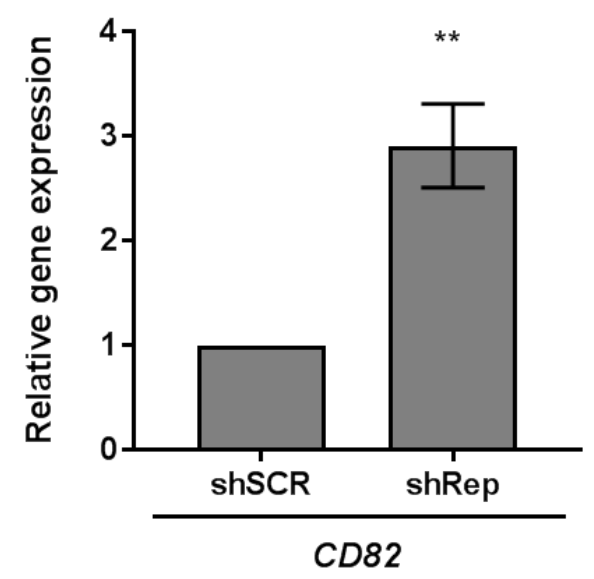

Figure 43. Reptin and CD82 transcript levels after 7 days of constitutive Reptin depletion.

qRT-PCR analysis of Reptin (A) and CD82 (B) expression levels in THP-1 cells constitutively expressing a control shRNA (shSCR) or a shRNA targeting Reptin (shRep). Columns represent the mean of three independent experiments and error bars represent SD. $P$-values comparing shRep samples to shSCR samples normalised to 1 were calculated using the one-sample $t$-test, $(* *) p \leq 0.01 ;(* * * *) p \leq 0.0001$. 


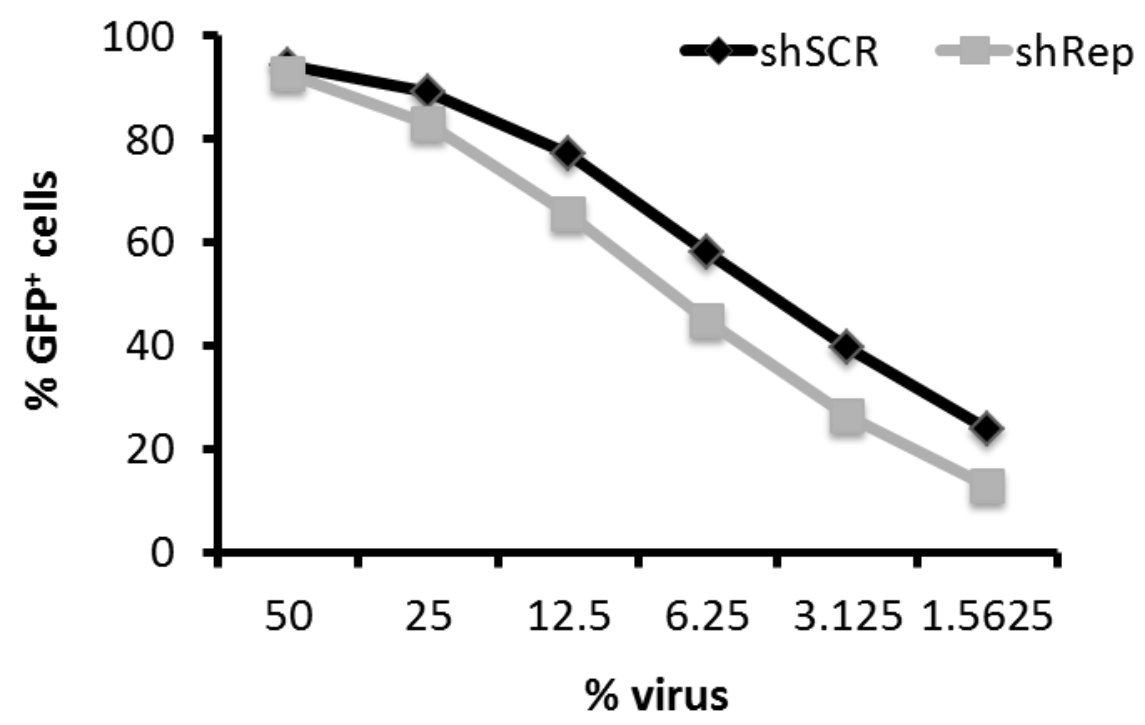

Figure 44. Measurement of virus titre prior to RNA-sequencing

Chart showing the measured viral titre for THP-1 cells transduced with a constitutive scrambled shRNA (shSCR) or a shRNA targeting Reptin (shRep). 3 days following transduction in 96 well plates with different percentages of virus supernatant, cells were fixed with $4 \%$ paraformaldehyde for 10 minutes and the percentage of GFP positive cells was measured using flow cytometry. These data were obtained from a single experiment. 
A)

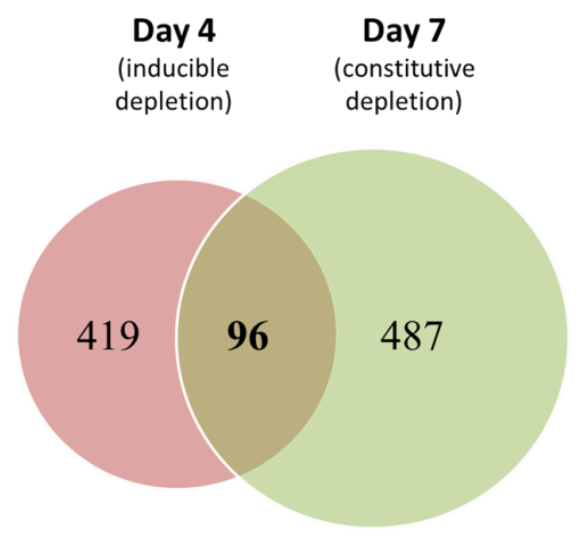

B) DOWNREGULATED

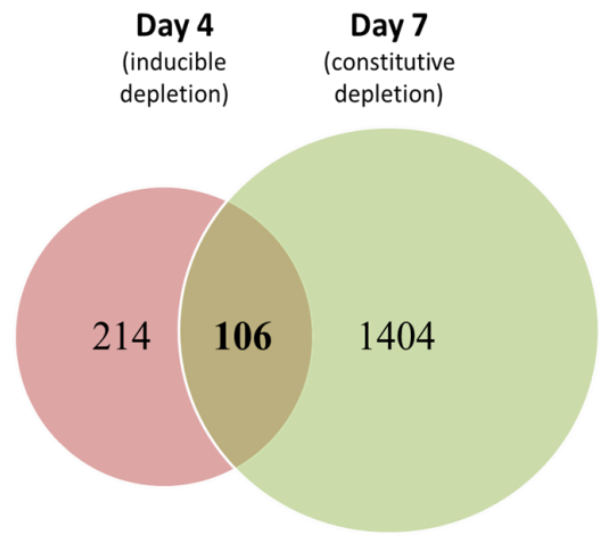

Figure 45. Upregulated and downregulated genes in common between two different RNA-seq experiments.

Venn diagrams representing the number of significant gene changes, 1.5 fold or above, in common between 2 different RNA-seq experiments after Reptin depletion, as indicated. Venn diagram (A) represents upregulated genes and diagram (B), downregulated genes. 
A)

B)
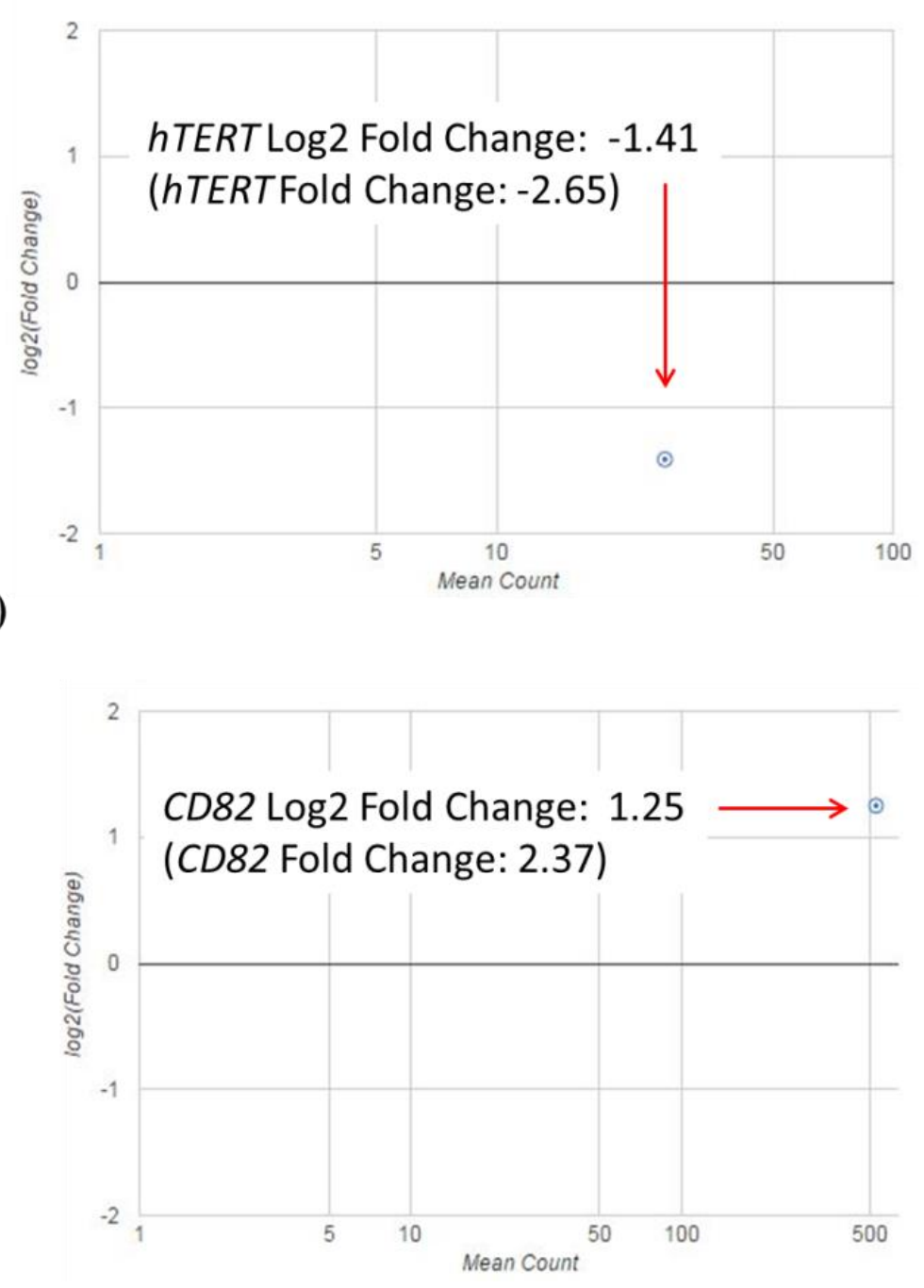

Figure 46. hTERT and CD82 $\log 2$ fold change after 7 days of constitutive Reptin depletion.

Scatter plots from Illumina Basespace showing the $\log 2$ fold change of hTERT (A) and CD82 (B) following 7 days of constitutive Reptin depletion, relative to control shSCR transduced cells. The scatter plots display the $\log 2$ fold change against the mean count of the gene. The circled dots represent the $h T E R T$ and $C D 82$ transcripts. 


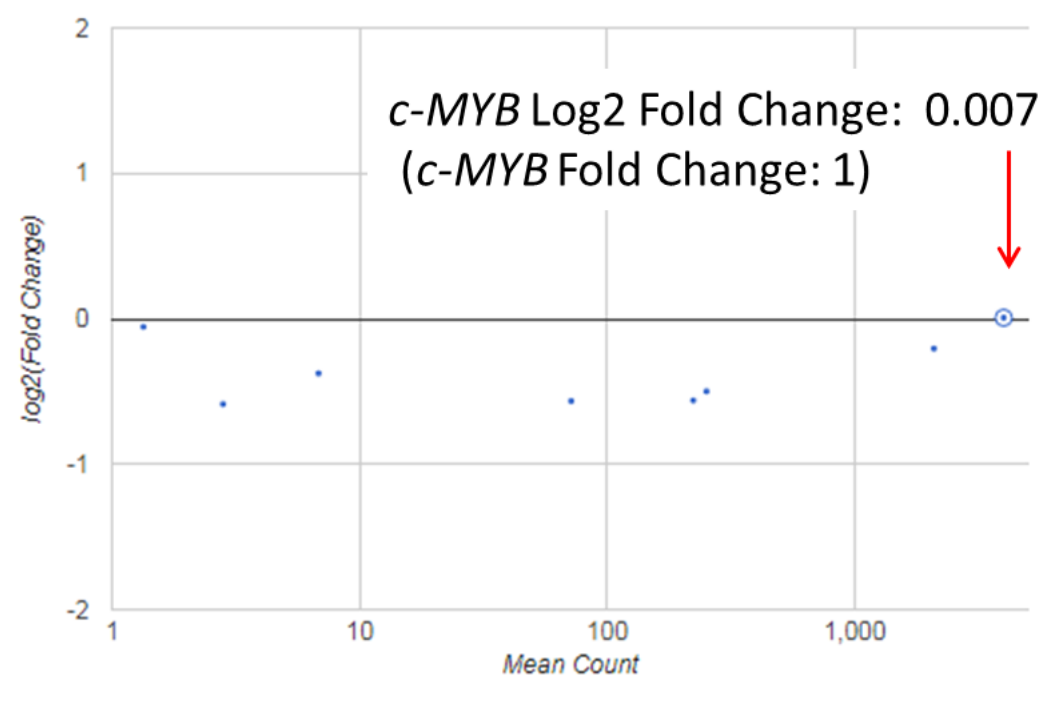

Figure 47. c-MYB transcript levels do not change after 7 days of constitutive Reptin depletion.

Scatter plot generated using the RNA Express tool of Illumina BasesPace showing the $\log 2$ fold change of the c-MYB transcript in THP-1 cells depleted of Reptin expression for 7 days. The scatter plot displays the $\log 2$ fold change against the mean count of a gene. Each dot represents a different gene and the circled dot represents $c$ $M Y B$. 

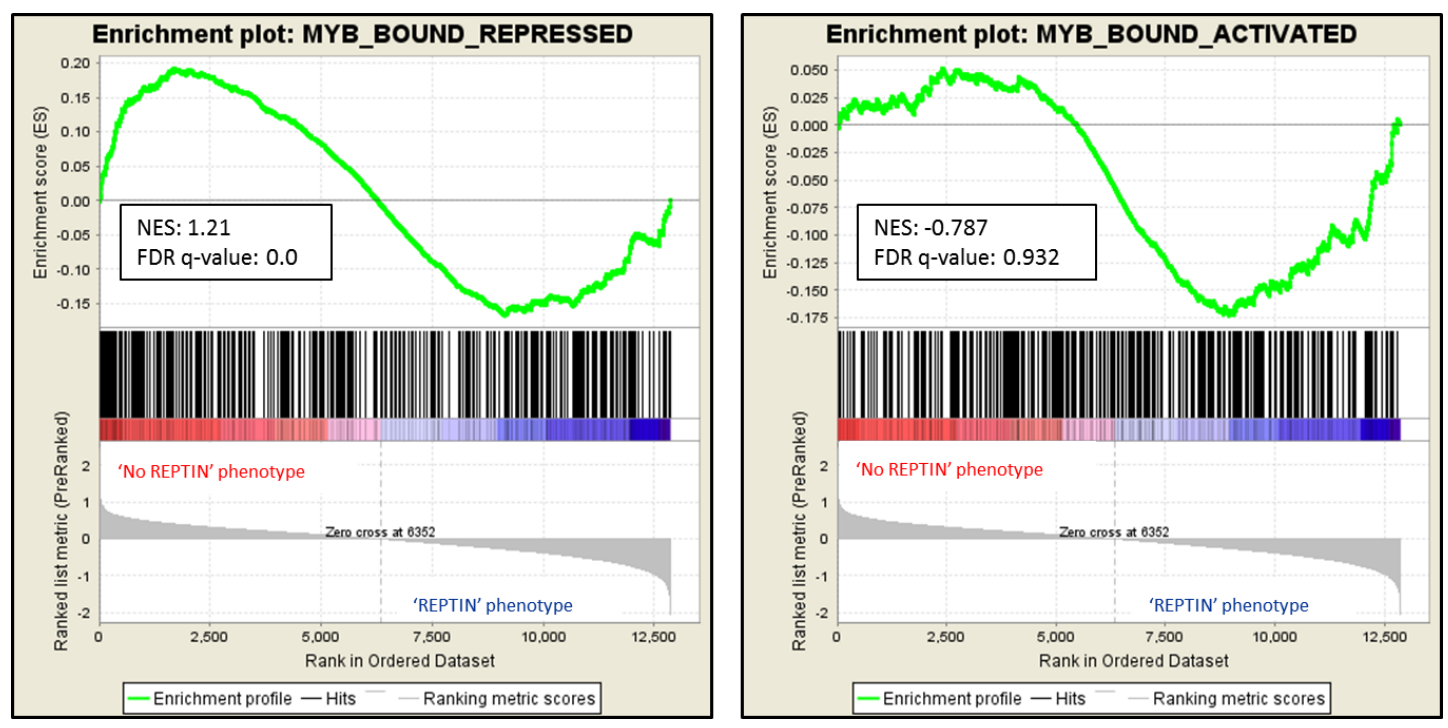

Figure 48. GSEA plots of the $c-M Y B$ signature after 7 days of constitutive Reptin depletion.

The figure shows GSEA enrichment plots of the c-MYB repressed (left) and activated (right) gene sets (Zhao et al., 2011) in Reptin depleted (NO REPTIN) vs. Reptin expressing (REPTIN) cells. NES, normalised enrichment score; FDR, false discovery rate. 


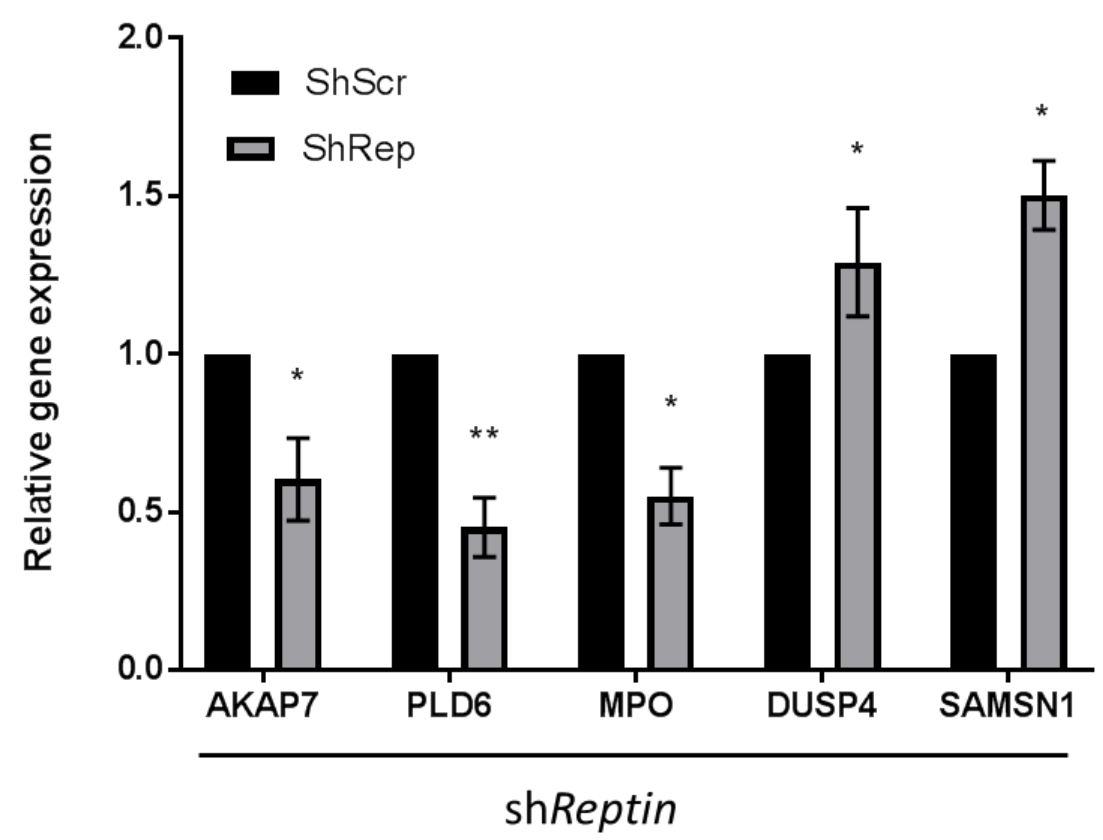

Figure 49. Validation of selected c-MYB target genes by qRT-PCR after constitutive Reptin depletion.

qRT-PCR analysis of c-MYB target genes in THP-1 cells depleted of Reptin expression for 7 days (pGIPZ vector), relative to the expression levels in control (shSCR) cells normalised to 1 . Bars represent the mean of three independent experiments and error bars represent SD. $P$-values comparing normalised shSCR to shRep samples were calculated using the one-sample $t$-test, $\left({ }^{* *}\right) p \leq 0.01 ;\left({ }^{*}\right) p \leq 0.05$; 
A)

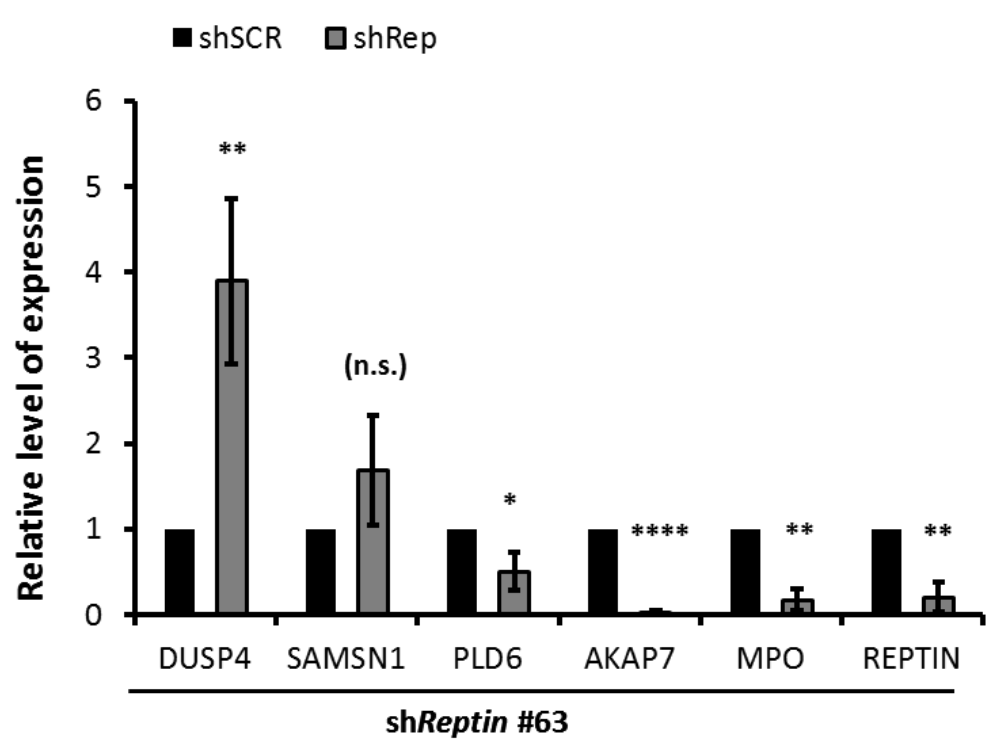

B) - shSCR $\square$ shRep

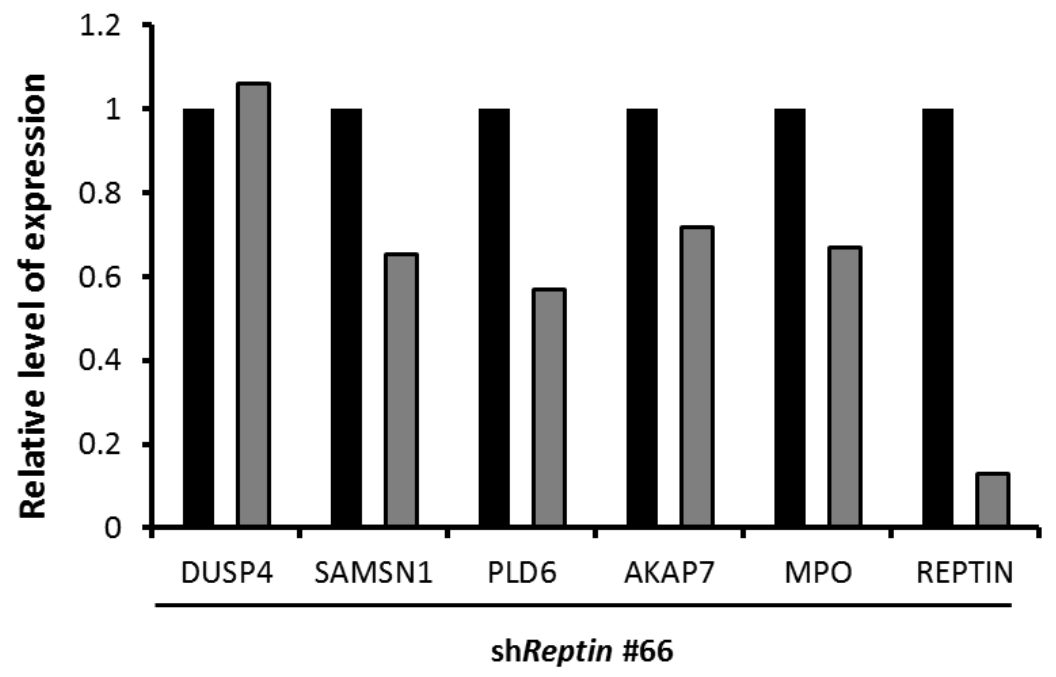

Figure 50. Validation of selected c-MYB target genes by qRT-PCR after constitutive Reptin depletion with 2 additional shRNA sequences.

qRT-PCR analysis of c-MYB target genes in THP-1 cells depleted of Reptin expression using two different MISSION® pLKO.1 shRNA sequences, \#63 shown in (A) and \#66 shown in (B). Data are shown relative to the expression levels in 
control (shSCR) cells normalised to 1. Reptin mRNA expression levels are also shown. For shRNA \#63, bars represent the mean of three (AKAP7, MPO) or four (DUSP4, SAMSN1, PLD6, Reptin) independent experiments and SD, the standard deviation. $P$-values comparing normalised shSCR to shRep samples were calculated using the one-sample $t$-test, $(* * * *) p \leq 0.001 ;(* *) p \leq 0.01 ;\left(^{*}\right) p \leq 0.05$; (n.s.), not significant. Bars for shRNA \#66, bars represent the SD of a single experiment. 


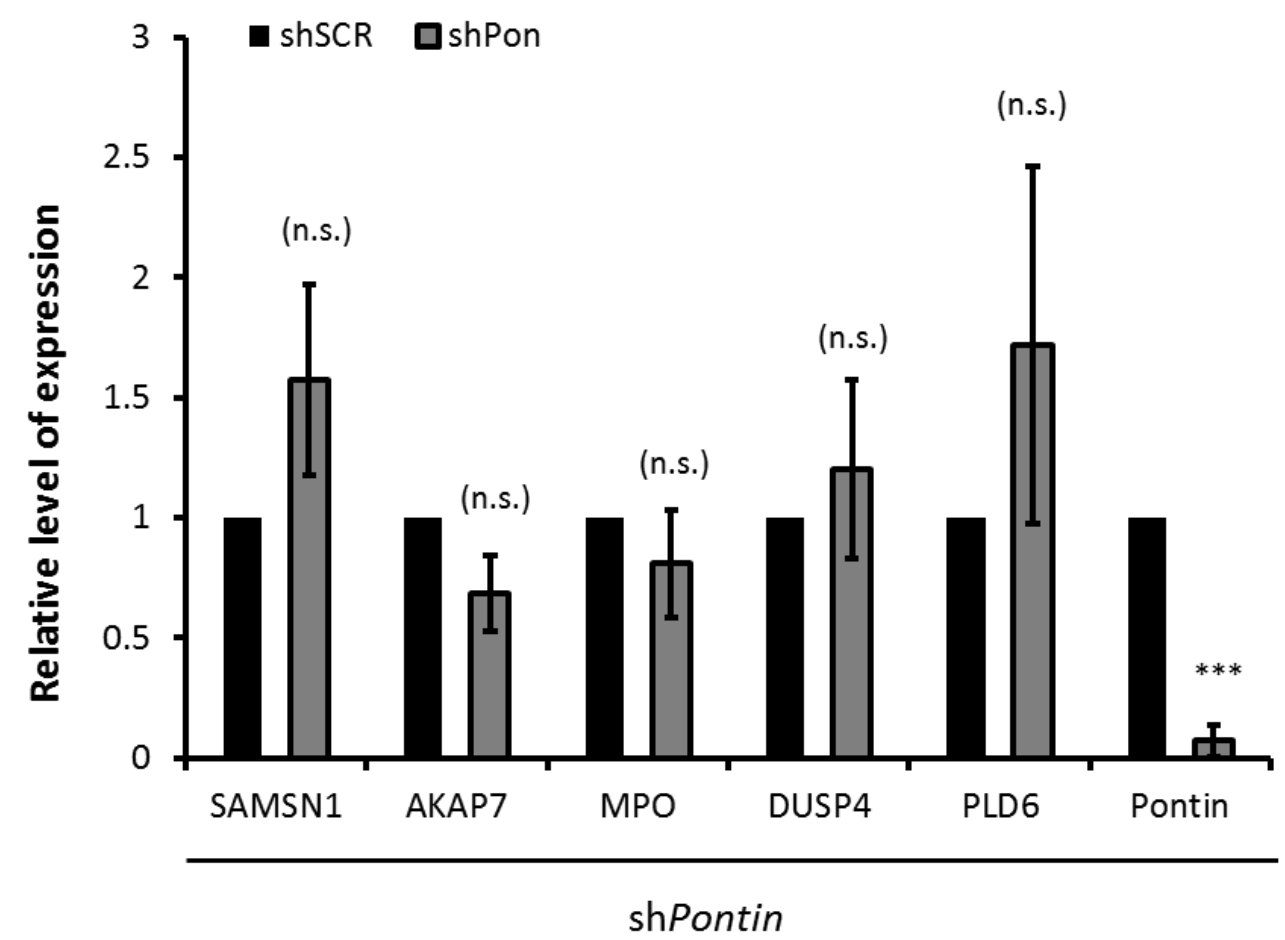

Figure 51. mRNA expression of c-MYB target genes after Pontin knockdown.

qRT-PCR analysis of c-MYB target genes in THP-1 cells depleted of Pontin expression. Data are shown relative to the expression levels in control (shSCR) cells normalised to 1. Pontin mRNA expression levels are also shown. Bars represent the mean of three independent experiments and error bars represent SD. $P$-values comparing normalised shSCR to shRep samples were calculated using the onesample $t$-test, $(* * *) p \leq 0.001 ;$ (n.s.), not significant. 


\subsubsection{Reptin interacts with c-MYB in AML cells}

Reptin has been found to modulate the transcriptional activity of several transcription factors through interaction and complex formation (Grigoletto et al., 2011). To assess whether Reptin modulates the c-MYB signature through complex formation, we performed immunoprecipitation assays to examine whether we could detect an interaction between Reptin and c-MYB in AML cells. We overexpressed the 3xHAtagged Reptin construct in 2 AML cell lines, THP-1 and U937, and immunoprecipitated with either an anti-IgG control antibody or an anti-c-MYB antibody. Immunoprecipitated lysates were subjected to western blot analysis and probed with an anti-HA antibody. Interestingly, we observed c-MYB and Reptin interaction in both cell lines (Figure 52). In order to confirm that this interaction could also be detected at endogenous levels, we used a control IgG antibody or an anti-Reptin antibody to immunoprecipitate endogenous Reptin in THP-1 cells. As shown in Figure 53, we detected c-MYB interaction in the Reptin immunoprecipitated lysates but not in the control ones (Figure 53). To confirm this finding, we repeated the immunoprecipitation assays in THP-1 cells depleted of cMYB expression. As shown in Figure 54, we did not observe c-MYB coimmunoprecipitation in c-MYB depleted cells, while we were still able to detect the interacting band in wild type THP-1 cells. Collectively, these results suggest that cMYB and Reptin can form complexes in AML cells at endogenous levels. 
A) THP-1 cells

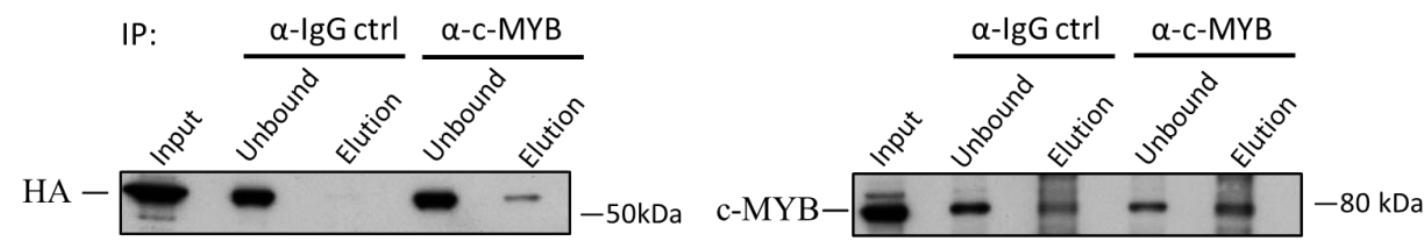

B) U937 cells
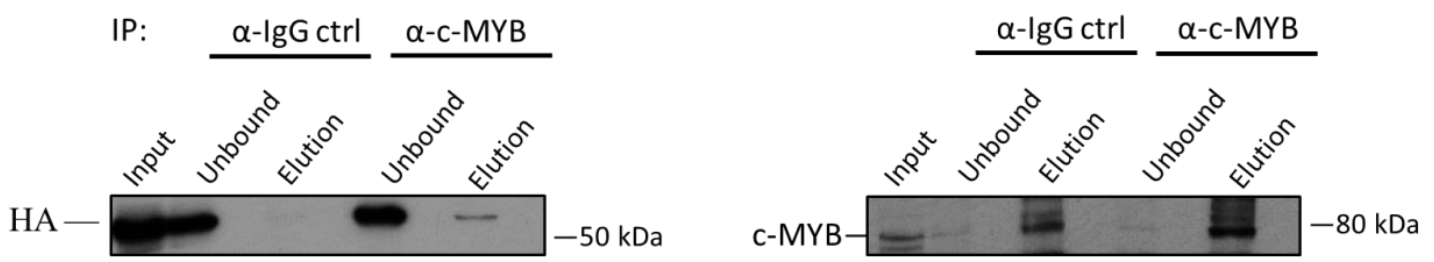

Figure 52. 3xHA-Reptin interacts with endogenous c-MYB in AML cell lines.

Cell lysates of THP-1 (A) or U937 (B) cells overexpressing 3xHA-Reptin were subjected to immunoprecipitation with an antibody against either c-MYB or control IgG. The resultant precipitates were immunoblotted with anti-HA or anti-c-MYB antibodies. "Input" refers to the total whole lysate. "Elution" refers to amount of protein co-immunoprecipitated by the specific antibody, whereas "Unbound" refers to the amount of protein not co-immunoprecipitated. The data are representative of three independent experiments. 

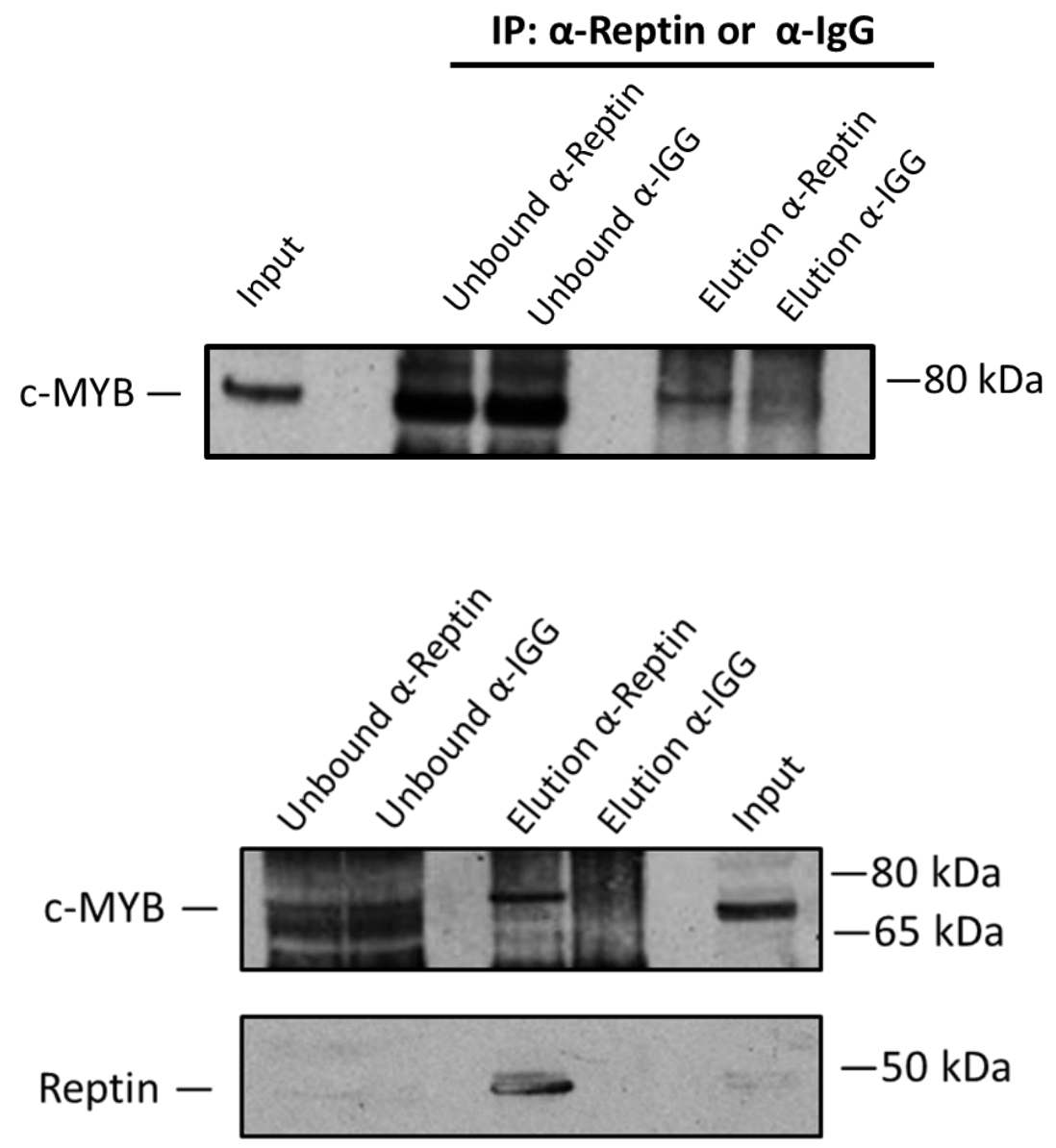

Figure 53. Endogenous Reptin protein interacts with endogenous c-MYB.

The figure shows two independent experiments of immunoprecipitation assays that prove the interaction of endogenous Reptin and c-MYB in THP-1 cells. THP-1 cell lysates were subjected to immunoprecipitation with control IgG or anti-Reptin antibodies. The resultant precipitates were immunoblotted with anti-Reptin or anti-cMYB antibodies. "Input" refers to the total whole lysate. "Elution" refers to amount of protein co-immunoprecipitated by the specific antibody, whereas "Unbound" refers to the amount of protein not co-immunoprecipitated. The data are representative of three independent experiments. 


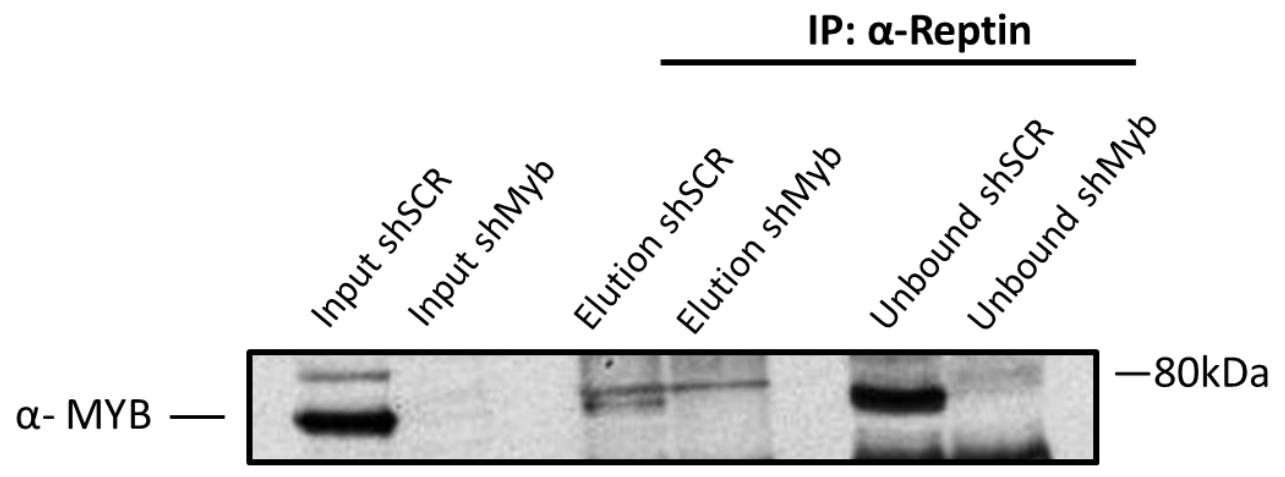

Figure 54. Endogenous Reptin immunoprecipitation in control and c-MYB depleted THP-1 cells.

THP-1 cells were transduced with either a control scrambled shRNA (shSCR) or a shRNA targeting c-MYB (shMYB). Cell lysates were subjected to immunoprecipitation with an anti-Reptin antibody. The resultant precipitates were immunoblotted with an anti-c-MYB antibody. "Input" refers to the total whole lysate. "Elution" refers to amount of protein co-immunoprecipitated by the specific antibody, whereas "Unbound" refers to the amount of protein not coimmunoprecipitated. The data are representative of three independent experiments. 


\subsubsection{Reptin decreases the binding of c-MYB to the chromatin}

Our data indicated that Reptin modulates the c-MYB genetic signature without affecting c-MYB transcript levels and that Reptin and c-MYB proteins interact in AML cells. Thus, we hypothesised that Reptin could be opening the chromatin conformation to facilitate c-MYB binding to promoters in a similar way it does for E2F1. Taragelo et al. recently described that, in hepatocellular carcinoma, E2F1 recruits Reptin and Pontin to incorporate the histone variant H2A.Z in low affinity E2F1 promoters. This modification opens the chromatin and amplifies the E2F1 transcriptional response (Tarangelo et al., 2015). In order to test our hypothesis, we performed c-MYB chromatin immunoprecipitation (ChIP) assays in control cells and in cells depleted of Reptin expression. Moreover, we also performed ChIP assays of H3K27ac, an open chromatin epigenetic mark. The ChIP assay allows identifying the presence of a transcription factor or histone protein bound at as specific region of the genome (Rodriguez-Ubreva and Ballestar, 2014). If our hypothesis is correct, this technique would allow us to see a decrease in c-MYB binding after Reptin loss, and a change in chromatin marks at c-MYB targets loci.

In order to perform ChIP assays we first optimised several conditions, such as the sequence and concentration of different primers. As we were uncertain of the exact regions in the genome of THP-1 cells where c-MYB binds, we tested several primers spanning promoter regions of different known c-MYB target genes. A table with the list of primers used in this study and a table with a list of primers tested can be found in the Materials and Methods section of this thesis (Table 11 and Table 12). 
Protein levels of c-MYB and Reptin in the cells used to perform ChIP assays, as measured by western blot analysis, are shown in Figure 55.

Remarkably, c-MYB binding at the promoter region of the well characterised target gene myeloperoxidase (MPO) was reduced on average by a third after Reptin depletion in comparison to control cells (Figure 56A). Our preliminary ChIP data of the active chromatin mark $\mathrm{H} 3 \mathrm{~K} 27 \mathrm{ac}$ also indicated decrease in the regulatory region of $M P O$ after Reptin depletion (Figure 56B). Collectively, these findings indicate that Reptin expression is necessary for the proper alteration of the chromatin and correct binding of $\mathrm{c}-\mathrm{MYB}$ at the promoter region of the MPO gene. Moreover, the data suggest the possibility that Reptin might be required for c-MYB binding at other target loci. 
A)

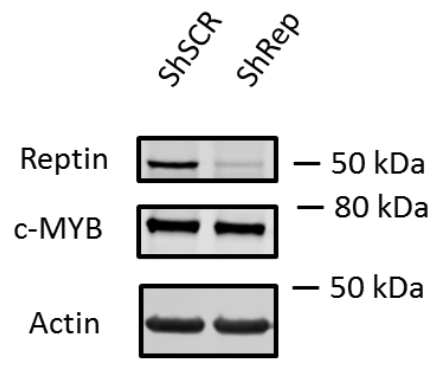

B)

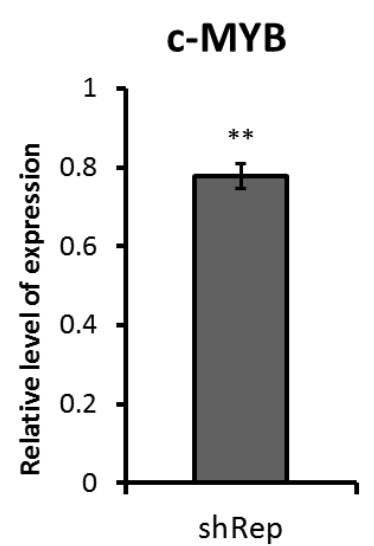

Reptin

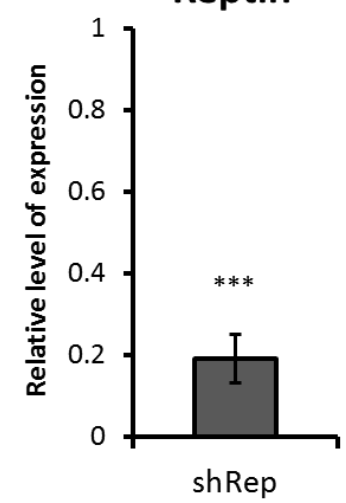

Figure 55. c-MYB and Reptin protein levels after Reptin depletion in the samples used for ChIP assays.

(A) Representative western blot analysis of c-MYB protein in the samples used to perform ChIP assays. THP-1 cells were transduced with constitutive scrambled shRNA (shSCR) and Reptin shRNA (shRep) for 6 days before taking lysates for analysis. Actin was used as a loading control. This experiment was performed 3 times. (B) The bar chart shows the densitometric quantification of c-MYB and Reptin protein, relative to Actin. Data represent the mean of three independent experiments and the error bars represent the SD. shSCR data were normalised to 1 and a $p$-value comparing shRep to normalised shSCR was calculated using the onesample $t$-test; $(* *) p \leq 0.01 ;(* * *) p \leq 0.001$. 
A)

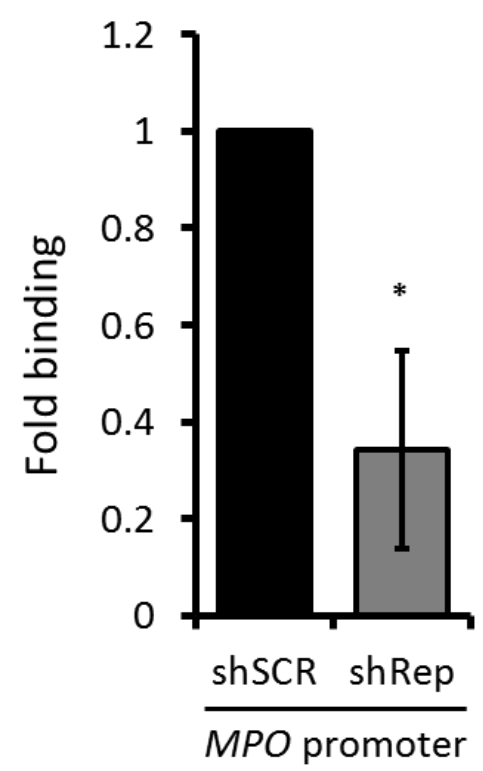

B) H3K27ac ChIP

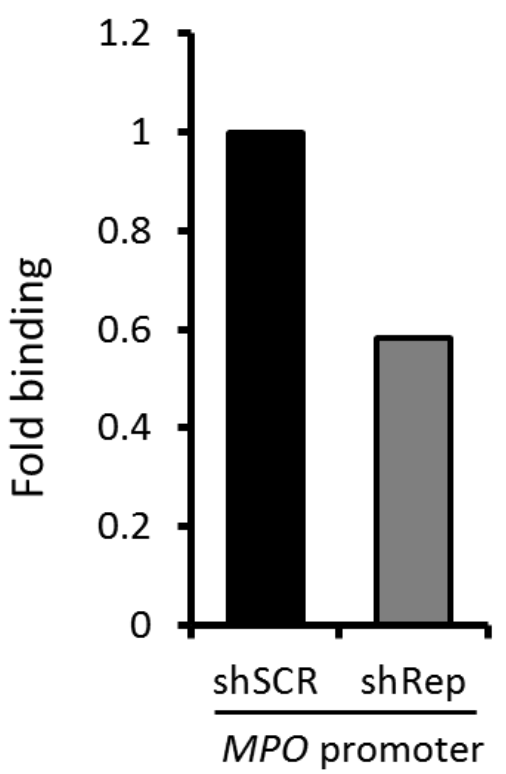

Figure 56. Reptin depletion results in decreased c-MYB and H3K27ac binding at the promoter region the $M P O$ gene.

ChIP assay to detect enrichment of c-MYB (A) and H3K27ac (B) at the promoter region of $M P O$ gene. THP-1 cells were transduced with a control scrambled shRNA (shSCR), or a shRNA targeting Reptin (shRep), and a qRT-PCR was performed with the immunoprecipitated chromatin. The binding was calculated relative to a control gene desert region in chromosome 21. Samples were also normalised to input DNA. Bars of the c-MYB ChIP represent the mean of three independent experiments and $\mathrm{SD}$, the standard deviation. shSCR data were normalised to 1 and a $p$-value comparing shRep to normalised shSCR was calculated using the one-sample $t$-test; (*) $p \leq 0.05$. Bars of the $\mathrm{H} 3 \mathrm{~K} 27 \mathrm{ac}$ represent data of a single experiment. 


\subsection{Discussion}

Our global transcriptome analysis after depleting Reptin in an inducible manner revealed a negative correlation of the $c-M Y B$ signature. In this chapter we have validated the global gene expression data by qRT-PCR, using a representative group of c-MYB upregulated and downregulated genes after Reptin depletion. In order to rule out the possibility that the results observed are a consequence of doxycycline treatment, we have further validated our results by generating additional gene expression data in AML cells expressing a constitutive Reptin-targeting shRNA. Even though our additional GSEA data of the constitutively depleted cells have only shown a significant result for c-MYB repressed genes, we have been able to confirm by qRT-PCR a significant change in the expression of both c-MYB activated and repressed genes after Reptin depletion. It is possible that we were not able to detect the effect of Reptin on c-MYB activated genes in our validation sequencing experiment due to the increased background noise that exists when a constitutive system is used, in comparison to the inducible system. The increased background noise may result from the effect that the virus transduction and the presence of the selection drug have on the cells and their gene expression.

Furthermore, in this chapter we have also validated a significant change of the selected c-MYB target genes with two additional shRNA sequences to deplete Reptin expression, indicating that our results are gene specific and not an off-target activity. Interestingly, when we looked at the effect of Pontin depletion on the selected c-MYB target genes, we did not get a significant change, suggesting that 
Reptin and Pontin might not be working together in the regulation of c-MYB function.

Collectively, all of our validation experiments confirmed that Reptin modulates the c-MYB signature in the $M L L$-rearranged AML cell line THP-1. Given that Reptin was also found necessary for the proliferation of other non-MLL rearranged AML cell types (Osaki et al., 2013), it would be interesting to determine whether the expression of c-MYB-regulated genes is also dependent on Reptin expression in these other leukaemic cell types. As the genes regulated by c-MYB might be different in different cells, a comparison of gene expression data obtained after Reptin depletion and after $c-M Y B$ depletion in different cell lines would provide the best answer. Since c-MYB has an essential role in maintaining the leukaemic phenotype initiated by a diverse group of genomic translocations (Pattabiraman and Gonda, 2013), it is plausible that the deleterious effect of Reptin depletion on those cell types might be due to a similar role of Reptin in regulating c-MYB-controlled gene expression.

Reptin was found to interact and directly regulate the function of several transcription factors, including c-MYC, E2F1, $\beta$-catenin and ATF2 (Gallant, 2007) (Tarangelo et al., 2015). Taking into account these published observations, and considering that we found a negative correlation of the c-MYB signature after Reptin depletion even when c-MYB levels remained unchanged, we hypothesised that Reptin could be modulating c-MYB function by forming a complex with it. To explore this possibility, we performed co-immunoprecipitation assays of c-MYB in 
AML cells overexpressing an HA-tagged form of Reptin. Interestingly, our results revealed a physical interaction between the overexpressed HA-tagged Reptin and cMYB in two different AML cell lines, suggesting that Reptin could also be modulating c-MYB function in non-MLL-rearranged human leukaemic cells. As previously mentioned, it remains to be elucidated whether the c-MYB signature is also affected by Reptin loss in other AML cell types. To validate that this interaction also takes place at endogenous levels, we immunoprecipitated endogenous Reptin in THP-1 cells and confirmed the interaction with endogenous c-MYB. In contrast to the interaction between Reptin and Pontin that we have shown in the previous chapter, in which most of Reptin was found to interact with Pontin, we only observed a small proportion of Reptin bound to c-MYB. This result would be consistent with an independent role, or even antagonistic role, of Reptin and Pontin in the regulation of c-MYB, as different examples in the literature have shown these dissimilarities in gene regulation by Reptin and Pontin (Bauer et al., 2000) (Kim et al., 2005). In one study, Pontin was found to activate the expression of the CD82/KAI1 gene by forming a complex with TIP60, while Reptin repressed its expression by interacting with $\beta$-catenin (Kim et al., 2005). Interestingly, TIP60 was found to interact with cMYB in human leukaemic cells and antagonise the transcriptional activation function of c-MYB by recruiting histone deacetylase (HDAC) proteins, which are associated with condensed chromatin and gene silencing (Zhao et al., 2012). It is then possible that in leukaemic cells TIP60 antagonises c-MYB function while Reptin, alone or in combination with other co-factors, facilitates c-MYB transcriptional activity. It would be intriguing to determine by immunoprecipitation assays whether Pontin also interacts with c-MYB in AML cells, and whether the complex formed by Reptin and 
c-MYB also includes other co-factors. For example, it would also be interesting to investigate whether the c-MYB co-activators $\mathrm{CBP} / \mathrm{p} 300$, which enhance c-MYB transcription by acetylating histone H3 and c-MYB (Greig et al., 2008), also interact with Reptin.

Recently, Reptin was described as a general amplifier of the transcriptional response of E2F1 in hepatocellular carcinoma (HCC). Reptin was found to open the chromatin conformation and facilitate the binding of E2F1 at low affinity binding promoters, which are not normally regulated by E2F1 in physiological conditions, by introducing the histone mark H2A.Z (Tarangelo et al., 2015). In fact, as mentioned in the introduction, Reptin forms part of several chromatin remodelling complexes, such as the INO80 or TIP60, which regulate gene transcription (Gallant, 2007). These observations made us hypothesise that Reptin could be modulating c-MYB transcriptional activity by modifying the chromatin and facilitating c-MYB binding to different promoters. To test this hypothesis we optimised the conditions for ChIP experiments and performed ChIP assays of c-MYB and the epigenetic mark H3K27ac in control and Reptin depleted THP-1 cells. Our c-MYB ChIP assays in Reptin depleted cells showed a marked decrease of c-MYB binding at the promoter region of the myeloperoxidase (MPO) gene, a well-known c-MYB target gene (Zhao et al., 2014), which we also found to be regulated by c-MYB in THP-1 cells. Although by western blot analysis we detected a small but significant decrease of cMYB protein in the samples used for ChIP assays, we believe this small decrease would not explain the striking reduction in c-MYB binding at the MPO promoter. As the half-life of c-MYB is very short (Ramsay and Gonda, 2008), it is possible that a 
small fraction of the protein might get degraded if it cannot correctly regulate gene transcription. Alternatively, as Reptin depletion leads to cell differentiation and apoptosis (Osaki et al., 2013), it is possible that the small effect seen on c-MYB protein might due to some cells starting to differentiate and die.

Nevertheless, our ChIP results indicate that Reptin activity is necessary for the biding of c-MYB to the $M P O$ promoter, and suggest the possibility that Reptin might be also necessary for the binding of c-MYB at the promoter region of other target genes. Moreover, Reptin depletion also decreased the binding of the H3K27ac, an active chromatin marker, on the proximal promoter region of $M P O$. Although this result is preliminary and more experiments should be performed, it suggests that Reptin might be necessary to modify the chromatin at the regulatory regions of cMYB target genes. Thus, it would be interesting to investigate whether other Reptinregulated chromatin markers, such as $\mathrm{H} 2 \mathrm{~A} . \mathrm{Z}$, or epigenetic marks of repressed transcription, such as $\mathrm{H} 3 \mathrm{~K} 27 \mathrm{me}$, are also altered at c-MYB transcriptional promoters after Reptin silencing (Jha and Dutta, 2009) (Zhao et al., 2011). As our gene expression analyses revealed a change in the expression of both c-MYB activated and repressed genes after Reptin depletion, our data suggest that Reptin might not only be necessary to open the chromatin at the promoters of activated cMYB target genes, such as $M P O$, but also to modify the chromatin at promoter regions of c-MYB repressed genes. To further investigate the role of Reptin on the regulation of c-MYB transcription, we have submitted our c-MYB and H3K27ac ChIP samples for sequencing to generate genome wide ChIP (ChIP-seq) data in control and Reptin depleted cells. We have obtained genome-wide peaks for c-MYB 
binding on the chromatin and we are planning to analyse the results with bioinformatics tools to investigate whether differential c-MYB binding is found after Reptin depletion. We envisage that the effect of Reptin depletion on c-MYB will be more global than just at the $M P O$ promoter, as our gene expression analyses indicated a negative correlation of the c-MYB signature. Importantly, our ChIP-seq analyses will also help us to determine whether Reptin is required to open up loci for all c-MYB regulated target genes or, similarly to what has been described in HCC with E2F1, just a subset of target genes not normally regulated by c-MYB, which are necessary for its leukaemogenic activity.

As well as having an essential role in maintaining the leukaemic phenotype (Pattabiraman and Gonda, 2013), c-MYB has been found to be deregulated in other non-hematopoietic tumours, mainly colon and breast cancer, but also melanoma, pancreatic cancer and oesophageal cancer (Ramsay and Gonda, 2008). For example, c-MYB was found to be overexpressed in colorectal cancer and this was correlated with poorer prognosis (Alitalo et al., 1984) (Ramsay et al., 1992) (Biroccio et al., 2001). In breast cancer, c-MYB expression was found to be correlated with the expression of the Oestrogen Receptor $\left(\mathrm{ER}^{+}\right)$and c-MYB silencing led to a decrease in the proliferation of $\mathrm{ER}^{+}$breast cancer cells (Guerin et al., 1990) (Drabsch et al., 2007). As Reptin and/or Pontin have also been found to be overexpressed in colon and breast cancer (Grigoletto et al., 2011) (Milone et al., 2016), it would be interesting to investigate whether Reptin and/or Pontin also interact with and modulate c-MYB transcriptional activity in these tumour types. 
In human leukaemia, c-MYB has been found to be a good target for therapy, as normal haematopoietic cells were found to be less sensitive to c-MYB suppression than leukaemic cells (Zuber et al., 2011). This suggested there might be a therapeutic window in which leukaemic cells can be targeted without affecting normal haematopoietic cells, which provided a rationale for developing novel therapies against c-MYB. However, therapeutic targeting of c-MYB has been challenging, as drugs targeting DNA-binding proteins are generally difficult to develop (Darnell, 2002). Several alternative methods have been tested, such as the use of antisense nucleic acids against c-MYB, or the development of small molecules that inhibit the elongation machinery necessary to transcribe c-MYB (Ramsay and Gonda, 2008). Recently, small molecule inhibitors that disrupt the interaction between c-MYB and its potent co-activator p300 have been discovered and have shown promising results in vitro and in vivo (Bujnicki et al., 2012) (Uttarkar et al., 2017). In line with this, if the complex formed by Reptin and c-MYB described in this study is essential for cMYB activity in AML, a novel approach to target c-MYB function could be the development of small molecules that inhibit this interaction. However, future studies are needed to better characterise the interaction between Reptin and c-MYB. It is possible that Reptin binds to c-MYB indirectly through a different cofactor. However, even if the interaction is direct, it still remains to be elucidated which domains of c-MYB and Reptin are required to form the complex. The domains could be identified by performing co-immunoprecipitation assays of tagged c-MYB and Reptin deletion mutants, in a similar approach to the one used by Wood et al. to identify the interacting domains between Pontin and c-MYC (Wood et al., 2000). In that study, Pontin was found to interact with c-MYC through the insertion domain 
(domain II) existing between the Walker A and Walker B motifs. Thus, it is possible that Reptin could also be interacting with c-MYB through this domain, but this should be investigated.

To identify compounds that disrupt the effect of Reptin on c-MYB transcriptional activity, a drug repurposing method could be used by interrogating the Connectivity Map (CMAP) database. The CMAP database includes a large number of genome-wide transcriptional expression data from different human cell lines treated with a diverse group of bioactive molecules. Through common geneexpression changes, it finds functional connections between drugs, genes and diseases to identify molecules against a particular pathway (Lamb et al., 2006). As it is possible that Reptin only modifies c-MYB occupancy at certain promoters, such as promoters with lower affinity, a combination of the RNA-seq and ChIP-seq results after Reptin depletion would be the best approach to interrogate the c-MAP database. This strategy would lead to the identification of compounds that may target the role of Reptin in modulating c-MYB activity, as Reptin might also be performing additional functions in the cell.

In summary, the results presented in this chapter identify a novel interaction between Reptin and c-MYB in AML. Moreover, our preliminary ChIP results provide evidence suggesting that c-MYB could be recruiting Reptin to its target loci to modify the chromatin and modulate c-MYB binding to the genome and its transcriptional activity (Figure 57). Thus, this interaction could be exploited to target c-MYB oncogenic function in AML. 


\section{Fusion oncoproteins}

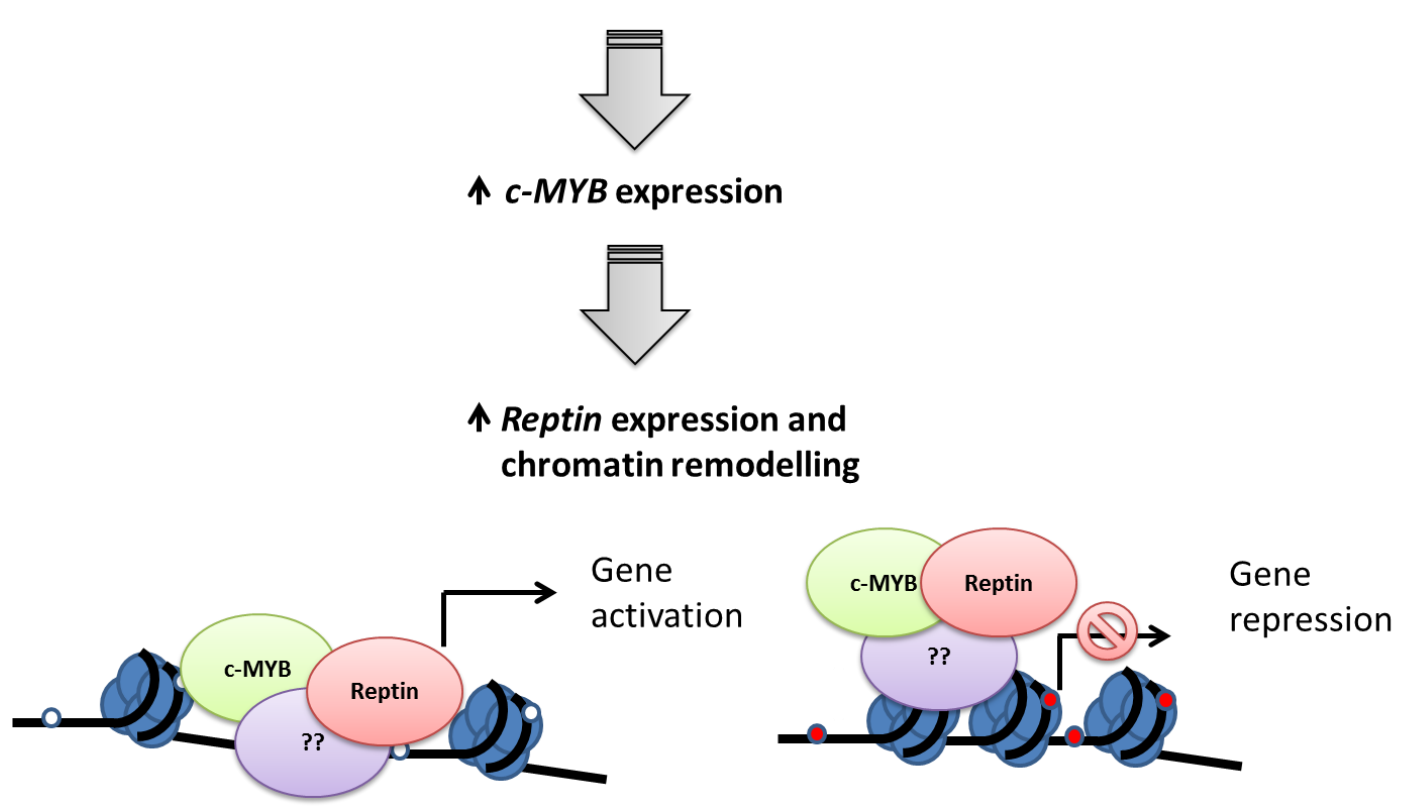

Figure 57. Proposed model of Reptin function in AML cells.

Chromosomal translocations that lead to fusion proteins increase $c-M Y B$ expression. In turn, c-MYB increases Reptin expression in AML cells. We propose that c-MYB recruits Reptin to its target promoters to facilitate chromatin remodelling. Chromatin changes at c-MYB regulated loci facilitate c-MYB mediated gene activation or repression. It is possible that additional co-factors forming a complex with c-MYB and Reptin facilitate this process. White and red dots on the chromatin represent different epigenetic marks. 


\section{CHAPTER VI - Conclusion}

Previous studies in our laboratory showed that Reptin expression in necessary for the proliferation, survival and clonogenic potential of immortalised human cord blood cells and of human AML cell lines (Osaki et al., 2013). In this thesis we aimed to elucidate the importance of Reptin expression for human leukaemia progression in vivo, as well as to investigate the transcriptional pathways modulated by Reptin in AML, to identify the mechanism of action that renders leukaemic cells dependent on Reptin expression.

In order to examine the role of Reptin in human AML progression in vivo, we xenotransplanted clones of the human AML cell line THP-1, previously generated in our group, to eliminate Reptin expression in an inducible manner. In these clones, Reptin is only depleted in the presence of doxycycline. Notably, Reptin depletion in established human leukaemia led to a significant increase in the survival of the doxycycline treated recipients, in comparison to control mice. Moreover, a complete remission of the disease was achieved in all of the recipients after Reptin loss, which was maintained in 5 out 6 mice even after doxycycline treatment was ceased. Similar results had previously been observed in mouse transplantations in a previous study led by our group (PhD thesis, Zhao, 2013). However, here we have described for the first time the relevance of Reptin expression for the maintenance of human leukaemia. Our results indicate that Reptin is necessary for disease progression and confirm that Reptin is a good target for therapy in human AML. 
To identify the molecular pathways regulated by Reptin in AML, we started by studying previously described Reptin interacting partners. Particularly, we focused our research on the oncogene c-MYC, as it was described to interact with Reptin (Wood et al., 2000), and previous research in our group identified a reduction in c-MYC protein after Reptin depletion ( $\mathrm{PhD}$ thesis, Zhao, 2013). Our data confirmed this reduction after Reptin silencing and after overexpressing an ATPase mutant of Reptin in THP-1 cells. Likewise, silencing the expression of other Reptin interacting partners, such as EPC1, EPC2 and MTBP (Huang et al., 2014) (Grieb et al., 2014) also led to a decrease in c-MYC protein levels. However, in contrast to previous observations made in our laboratory, in which the c-MYC transcript did not change after Reptin depletion ( $\mathrm{PhD}$ thesis, Zhao, 2013), our experiments showed a marked and significant decrease in $c-M Y C$ mRNA levels after Reptin loss. Moreover, also in contrast to previous published research (Wood et al., 2000), we were not able to detect an interaction between c-MYC and Reptin by immunoprecipitation assays. Thus, taken together these findings indicate that Reptin and its binding partner proteins are necessary to modulate the expression of the oncogenic protein c-MYC in AML, probably due to a decreased transcription of the $c-M Y C$ gene.

Next, we sought to determine the transcriptional pathways regulated by Reptin. To that end, we decided to use the bulk of the THP-1 cell line, rather than the clones, to avoid focusing on clone-specific transcriptional changes. After characterising the response of the bulk population upon Reptin loss, to confirm that similar Reptin knockdown and apoptosis levels than in the clones were achieved, we generated gene expression data after 2 and 4 days of Reptin depletion. These time 
points were chosen because they were early times points in which a noticeable decrease in Reptin protein expression was detected. Time point Day 2 was found to be too early to detect transcriptional gene changes. In contrast, after 4 days of Reptin depletion, several significant gene expression changes were detected. As we had previously observed a decrease in $c-M Y C$ transcript levels, and c-MYC is a known target gene of c-MYB in $M L L$-rearranged leukaemia (Hess et al., 2006), we used our gene expression data at Day 4 to examine the c-MYB signature by GSEA analysis. Notably, our analyses indicated a strong negative correlation of both activated and repressed c-MYB target genes. Following validation of the signature by qRT-PCR, we further validated our results by generating additional gene expression data of THP-1 cells constitutively depleted of Reptin, and by qRT-PCR using two additional shRNA sequences to deplete Reptin expression. In total, the results indicated that Reptin modulates the c-MYB signature in THP-1 cells. As Reptin performs many of its functions in conjunction with its homologous protein Pontin, we also analysed the expression of selected c-MYB target genes in Pontin depleted THP-1 cells. Our results did not indicate a clear and significant change in c-MYB regulated targets, suggesting that Pontin might not have the same function as Reptin in modulating cMYB activity in AML cells.

To further study the role of Reptin on c-MYB function, we performed immunoprecipitation assays of overexpressed and endogenous Reptin, and of endogenous c-MYB. Our results indicated that Reptin and c-MYB interact at both overexpressed and endogenous levels, suggesting that Reptin could be modulating cMYB function by forming a complex together. As Reptin forms part of several 
macromolecular complexes (Nano and Houry, 2013), it is possible that other factors might be also part of the complex involved in the regulation of c-MYB activity.

In a previous study, E2F1 was found to recruit Reptin and Pontin to low affinity promoters to open chromatin structure and amplify E2F1 transcriptional response in HCC (Tarangelo et al., 2015). As we found an interaction between Reptin and c-MYB, we hypothesised that c-MYB could be also recruiting Reptin to its target promoters in leukaemic cells to facilitate chromatin remodelling at its target loci, important for oncogenic transformation. To investigate this possibility, we performed ChIP assays of c-MYB and the epigenetic mark H3K27ac in control and Reptin depleted cells. Reptin loss decreased c-MYB binding at the promoter region of the $M P O$ gene by a third, in comparison to control cells. Moreover, preliminary ChIP data of the H3K27ac chromatin mark in Reptin depleted cells also indicated a decrease at the regulatory region of the $M P O$ promoter. As our gene expression data indicated a change in expression of the c-MYB signature, it is possible that Reptin might also be necessary to modify the chromatin conformation at other c-MYB target genes. Therefore, our results prompted us to submit our samples for ChIP-seq to obtain global and loci-specific binding data. Our future analyses of the ChIP-seq data will determine whether Reptin is necessary to facilitate the binding of c-MYB at all of its target promoters or just at specific loci, such as low binding affinity promoters necessary for the leukaemogenic activity of c-MYB. If this latter hypothesis is true, it would suggest that the role of Reptin in physiological conditions and in leukaemogenesis could be different, which could be exploited to target c-MYB oncogenic activity in AML. 
Earlier research in our group found that Reptin is regulated by c-MYB in $M L L$-rearranged AML, as $c-M Y B$ silencing in THP-1 cells resulted in decreased Reptin expression (Osaki, 2011). Moreover, other groups published similar results (Zuber et al., 2011) (Suzuki et al., 2009). Consistent with the data presented in this thesis, it is possible that c-MYB enhances Reptin expression in AML cells to facilitate its own transcriptional function, necessary for AML survival. This is not uncommon among transcriptional regulators. For example, c-MYB is a well characterised downstream transcriptional target of the $M L L$-fusion genes, and it was found to be required for MLL-driven leukaemogenesis by forming a complex with the MLL-fusion proteins through Menin (Jin et al., 2010). Thus, a similar phenomenon could be occurring for c-MYB and Reptin in AML cells.

To the best of our knowledge, this is the first study that identifies an interaction between Reptin and c-MYB, and that shows a role for Reptin in the regulation of c-MYB transcriptional activity. Thus, our findings expand the role of Reptin-regulated pathways to include the c-MYB pathway, at least in human $M L L$ rearranged AML. Here we propose that, in $M L L$-rearranged leukaemia, deregulated c-MYB increases Reptin expression to induce chromatin remodelling changes, and to facilitate c-MYB binding and function at the regulatory sites of its target genes (Figure 57). In this study we have found that Reptin modulates both c-MYB repressed and activated genes, although several examples in the literature show Reptin as a repressor, for example at the KAIl locus (Kim et al., 2005) or in the modulation of $\beta$-catenin target genes (Bauer et al., 2000). As Reptin is important for the proliferation and survival of several cancer types (Grigoletto et al., 2011) and 
modulates the function of different transcription factors (Gallant, 2007), it possible that the repressing or activating function of Reptin and the transcription factor which it regulates might depend on the cellular context. Thus, Reptin might be necessary to facilitate the abnormal function of the critical transcription factors in every particular transformed tissue. For example, while Reptin regulates the function of E2F1 in $\mathrm{HCC}$, in AML it may be necessary to regulate altered c-MYB activity because this is a critical transcription factor for the survival of these leukaemias.

The in vivo data in chapter III and the results obtained with the dominant negative mutant of Reptin in chapter IV suggested that targeting the ATPase domain of Reptin would be a promising therapeutic option. Moreover, the overexpression of a dominant negative mutant of Reptin was found to have a limited impact on normal mouse HPC colony formation, but to reduce leukaemia progression in vivo $(\mathrm{PhD}$ thesis, Zhao, 2013). These results indicate that a therapeutic window might exist, in which Reptin could be targeted in transformed cells without affecting normal progenitor cells. However, it should be noted that the Walker A + B motifs of Reptin and Pontin are almost identical, making it highly likely that drugs targeting the ATPAse domain of Reptin, would also inhibit Pontin. This may pose a problem, since conditional deletion of Pontin in the haematopoietic system of mice resulted in bone marrow failure and death (Bereshchenko et al., 2012) (Rajendra et al., 2014). Moreover, it remains to be elucidated what effect targeting the ATPase domain of Reptin would have on its function in regulating c-MYB. An alternative is suggested by our experiments in chapter V demonstrating an interaction of Reptin with c-MYB, as c-MYB has been proposed to be a good target for therapy in AML (Zuber et al., 
2011). This interaction is likely to be through domains outside the Walker motifs, for example the insertion domain, as in the case for c-MYC binding (Wood et al., 2000). Thus, impairing the interaction formed by Reptin and c-MYB may represent an alternative novel surrogate therapeutic strategy for c-MYB targeting in human AML. 


\section{CHAPTER VII - References}

ADOLFSSON, J., BORGE, O. J., BRYDER, D., THEILGAARD-MONCH, K., ASTRAND-GRUNDSTROM, I., SITNICKA, E., SASAKI, Y. \& JACOBSEN, S. E. 2001. Upregulation of Flt3 expression within the bone marrow Lin(-)Sca1(+)c-kit(+) stem cell compartment is accompanied by loss of self-renewal capacity. Immunity, 15, 659-69.

ADOLFSSON, J., MANSSON, R., BUZA-VIDAS, N., HULTQUIST, A., LIUBA, K., JENSEN, C. T., BRYDER, D., YANG, L., BORGE, O. J., THOREN, L. A., ANDERSON, K., SITNICKA, E., SASAKI, Y., SIGVARDSSON, M. \& JACOBSEN, S. E. 2005. Identification of Flt3+ lympho-myeloid stem cells lacking erythro-megakaryocytic potential a revised road map for adult blood lineage commitment. Cell, 121, 295-306.

AKASHI, K., TRAVER, D., MIYAMOTO, T. \& WEISSMAN, I. L. 2000. A clonogenic common myeloid progenitor that gives rise to all myeloid lineages. Nature, 404, 193-7.

ALITALO, K., WINQVIST, R., LIN, C. C., DE LA CHAPELLE, A., SCHWAB, M. \& BISHOP, J. M. 1984. Aberrant expression of an amplified c-myb oncogene in two cell lines from a colon carcinoma. Proc Natl Acad Sci U S A, 81, 45348.

AMANULLAH, A., LIEBERMANN, D. A. \& HOFFMAN, B. 2000. p53independent apoptosis associated with c-Myc-mediated block in myeloid cell differentiation. Oncogene, 19, 2967-77.

AMANULLAH, A., LIEBERMANN, D. A. \& HOFFMAN, B. 2002. Deregulated cMyc prematurely recruits both Type I and II CD95/Fas apoptotic pathways associated with terminal myeloid differentiation. Oncogene, 21, 1600-10.

AMATI, B., BROOKS, M. W., LEVY, N., LITTLEWOOD, T. D., EVAN, G. I. \& LAND, H. 1993. Oncogenic activity of the c-Myc protein requires dimerization with Max. Cell, 72, 233-45.

ANDERS, S. \& HUBER, W. 2010. Differential expression analysis for sequence count data. Genome Biol, 11, R106.

ANFOSSI, G., GEWIRTZ, A. M. \& CALABRETTA, B. 1989. An oligomer complementary to c-myb-encoded mRNA inhibits proliferation of human myeloid leukemia cell lines. Proc Natl Acad Sci U S A, 86, 3379-83.

ANJOS-AFONSO, F., CURRIE, E., PALMER, H. G., FOSTER, K. E., TAUSSIG, D. C. \& BONNET, D. 2013. CD34(-) cells at the apex of the human hematopoietic stem cell hierarchy have distinctive cellular and molecular signatures. Cell Stem Cell, 13, 161-74.

ARBER, D. A., ORAZI, A., HASSERJIAN, R., THIELE, J., BOROWITZ, M. J., LE BEAU, M. M., BLOOMFIELD, C. D., CAZZOLA, M. \& VARDIMAN, J. W. 2016. The 2016 revision to the World Health Organization classification of myeloid neoplasms and acute leukemia. Blood, 127, 2391-405.

ARMSTRONG, S. A., STAUNTON, J. E., SILVERMAN, L. B., PIETERS, R., DEN BOER, M. L., MINDEN, M. D., SALLAN, S. E., LANDER, E. S., GOLUB, T. R. \& KORSMEYER, S. J. 2002. MLL translocations specify a distinct gene expression profile that distinguishes a unique leukemia. Nat Genet, 30, 41-7. 
ARNOLD, C. N., PIRIE, E., DOSENOVIC, P., MCINERNEY, G. M., XIA, Y., WANG, N., LI, X., SIGGS, O. M., KARLSSON HEDESTAM, G. B. \& BEUTLER, B. 2012. A forward genetic screen reveals roles for Nfkbid, Zeb1, and Ruvb12 in humoral immunity. Proc Natl Acad Sci U S A, 109, 12286-93.

AYTON, P. M. \& CLEARY, M. L. 2003. Transformation of myeloid progenitors by MLL oncoproteins is dependent on Hoxa7 and Hoxa9. Genes Dev, 17, 2298307.

BAHRAM, F., WU, S., OBERG, F., LUSCHER, B. \& LARSSON, L. G. 1999. Posttranslational regulation of Myc function in response to phorbol ester/interferon-gamma-induced differentiation of v-Myc-transformed U-937 monoblasts. Blood, 93, 3900-12.

BAUER, A., CHAUVET, S., HUBER, O., USSEGLIO, F., ROTHBACHER, U., ARAGNOL, D., KEMLER, R. \& PRADEL, J. 2000. Pontin52 and reptin52 function as antagonistic regulators of beta-catenin signalling activity. Embo j, 19, 6121-30.

BAUER, A., HUBER, O. \& KEMLER, R. 1998. Pontin52, an interaction partner of beta-catenin, binds to the TATA box binding protein. Proc Natl Acad Sci U S A, 95, 14787-92.

BELlONI, E., SHING, D., TAPINASSI, C., VIALE, A., MANCUSO, P., MALAZZI, O., GERBINO, E., DALL'OLIO, V., EGURBIDE, I., ODERO, M. D., BERTOLINI, F. \& PELICCI, P. G. 2011. In vivo expression of an aberrant MYB-GATA1 fusion induces leukemia in the presence of GATA1 reduced levels. Leukemia, 25, 733-6.

BEllosta, P., HUlF, T., BALlA DIOP, S., USSEGLIO, F., PRADEL, J., ARAGNOL, D. \& GALLANT, P. 2005. Myc interacts genetically with Tip48/Reptin and Tip49/Pontin to control growth and proliferation during Drosophila development. Proc Natl Acad Sci U S A, 102, 11799-804.

BERESHCHENKO, O., MANCINI, E., LUCIANI, L., GAMBARDELLA, A., RICCARDI, C. \& NERLOV, C. 2012. Pontin is essential for murine hematopoietic stem cell survival. Haematologica, 97, 1291-4.

BEVERLY, L. J. \& VARMUS, H. E. 2009. MYC-induced myeloid leukemogenesis is accelerated by all six members of the antiapoptotic BCL family. Oncogene, 28, 1274-9.

BIEDENKAPP, H., BORGMEYER, U., SIPPEL, A. E. \& KLEMPNAUER, K. H. 1988. Viral myb oncogene encodes a sequence-specific DNA-binding activity. Nature, 335, 835-7.

BIROCCIO, A., BENASSI, B., D'AGNANO, I., D'ANGELO, C., BUGLIONI, S., MOTTOLESE, M., RICCIOTTI, A., CITRO, G., COSIMELli, M., RAMSAY, R. G., CALABRETTA, B. \& ZUPI, G. 2001. c-Myb and Bcl-x overexpression predicts poor prognosis in colorectal cancer: clinical and experimental findings. Am J Pathol, 158, 1289-99.

BLACKWELL, T. K., KRETZNER, L., BLACKWOOD, E. M., EISENMAN, R. N. \& WEINTRAUB, H. 1990. Sequence-specific DNA binding by the c-Myc protein. Science, 250, 1149-51.

BLACKWOOD, E. M. \& EISENMAN, R. N. 1991. Max: a helix-loop-helix zipper protein that forms a sequence-specific DNA-binding complex with Myc. Science, 251, 1211-7. 
BLAIR, A., HOGGE, D. E. \& SUTHERLAND, H. J. 1998. Most acute myeloid leukemia progenitor cells with long-term proliferative ability in vitro and in vivo have the phenotype CD34(+)/CD71(-)/HLA-DR. Blood, 92, 4325-35.

BLANC, J. F., LALANNE, C., PLOMION, C., SCHMITTER, J. M., BATHANY, K., GION, J. M., BIOULAC-SAGE, P., BALABAUD, C., BONNEU, M. \& ROSENBAUM, J. 2005. Proteomic analysis of differentially expressed proteins in hepatocellular carcinoma developed in patients with chronic viral hepatitis C. Proteomics, 5, 3778-89.

BONNET, D. 2005. Normal and leukaemic stem cells. Br J Haematol, 130, 469-79.

BONNET, D. \& DICK, J. E. 1997. Human acute myeloid leukemia is organized as a hierarchy that originates from a primitive hematopoietic cell. Nat Med, 3, 730-7.

BREIG, O., BRAS, S., MARTINEZ SORIA, N., OSMAN, D., HEIDENREICH, O., HAENLIN, M. \& WALTZER, L. 2014. Pontin is a critical regulator for AML1-ETO-induced leukemia. Leukemia, 28, 1271-9.

BREIG, O., YATES, M., NEAUD, V., COUCHY, G., GRIGOLETTO, A., LUCCHESI, C., PROX, J., ZUCMAN-ROSSI, J., BECKER-PAULY, C. \& ROSENBAUM, J. 2017. Metalloproteinase meprin alpha regulates migration and invasion of human hepatocarcinoma cells and is a mediator of the oncoprotein Reptin. Oncotarget, 8, 7839-7851.

BROGNA, S. \& WEN, J. 2009. Nonsense-mediated mRNA decay (NMD) mechanisms. Nat Struct Mol Biol, 16, 107-13.

BUJNICKI, T., WILCZEK, C., SCHOMBURG, C., FELDMANN, F., SCHLENKE, P., MULLER-TIDOW, C., SCHMIDT, T. J. \& KLEMPNAUER, K. H. 2012. Inhibition of Myb-dependent gene expression by the sesquiterpene lactone mexicanin-I. Leukemia, 26, 615-22.

CALABRETTA, B., SIMS, R. B., VALTIERI, M., CARACCIOLO, D., SZCZYLIK, C., VENTURELLI, D., RATAJCZAK, M., BERAN, M. \& GEWIRTZ, A. M. 1991. Normal and leukemic hematopoietic cells manifest differential sensitivity to inhibitory effects of c-myb antisense oligodeoxynucleotides: an in vitro study relevant to bone marrow purging. Proc Natl Acad Sci U S A, 88, 2351-5.

CARLSON, M. L., WILSON, E. T. \& PRESCOTT, S. M. 2003. Regulation of COX2 transcription in a colon cancer cell line by Pontin52/TIP49a. Mol Cancer, 2, 42.

CARPINELli, M. R., HILTON, D. J., METCALF, D., ANTONCHUK, J. L., HYLAND, C. D., MIFSUD, S. L., DI RAGO, L., HILTON, A. A., WILLSON, T. A., ROBERTS, A. W., RAMSAY, R. G., NICOLA, N. A. \& ALEXANDER, W. S. 2004. Suppressor screen in Mpl-/- mice: c-Myb mutation causes supraphysiological production of platelets in the absence of thrombopoietin signaling. Proc Natl Acad Sci U S A, 101, 6553-8.

CARRIE N. ARNOLD, E. P., EVA MARIE Y. MORESCO, ANNE MURRAY, BEUTLER B. 2016. Record for Worker, MUTAGENETIX (TM) [Online]. Dallas, TX.: Center for the Genetics of Host Defense, UT Southwestern Medical Center,

CASLINI, C., YANG, Z., EL-OSTA, M., MILNE, T. A., SLANY, R. K. \& HESS, J. L. 2007. Interaction of MLL amino terminal sequences with menin is required for transformation. Cancer Res, 67, 7275-83. 
CHEN, L., SUN, Y., WANG, J., JIANG, H. \& MUNTEAN, A. G. 2016. Differential regulation of the c-Myc/Lin28 axis discriminates subclasses of rearranged MLL leukemia. Oncotarget, 7, 25208-23.

CHO, S. G., BHOUMIK, A., BRODAY, L., IVANOV, V., ROSENSTEIN, B. \& RONAI, Z. 2001. TIP49b, a regulator of activating transcription factor 2 response to stress and DNA damage. Mol Cell Biol, 21, 8398-413.

CHOI, J., HEO, K. \& AN, W. 2009. Cooperative action of TIP48 and TIP49 in H2A.Z exchange catalyzed by acetylation of nucleosomal H2A. Nucleic Acids Res, 37, 5993-6007.

CLAPPIER, E., CUCCUINI, W., KALOTA, A., CRINQUETTE, A., CAYUELA, J. M., DIK, W. A., LANGERAK, A. W., MONTPELlIER, B., NADEL, B., WALRAFEN, P., DELATTRE, O., AURIAS, A., LEBLANC, T., DOMBRET, H., GEWIRTZ, A. M., BARUCHEL, A., SIGAUX, F. \& SOULIER, J. 2007. The C-MYB locus is involved in chromosomal translocation and genomic duplications in human T-cell acute leukemia (TALL), the translocation defining a new T-ALL subtype in very young children. Blood, 110, 1251-61.

CLARKE, D., VEGIOPOULOS, A., CRAWFORD, A., MUCENSKI, M., BONIFER, C. \& FRAMPTON, J. 2000. In vitro differentiation of c-myb(-/-) ES cells reveals that the colony forming capacity of unilineage macrophage precursors and myeloid progenitor commitment are c-Myb independent. Oncogene, 19, 3343-51.

CLARKE, M. F., KUKOWSKA-LATALlO, J. F., WESTIN, E., SMITH, M. \& PROCHOWNIK, E. V. 1988. Constitutive expression of a c-myb cDNA blocks Friend murine erythroleukemia cell differentiation. Mol Cell Biol, 8, 884-92.

CLOUTIER, P., POITRAS, C., DURAND, M., HEKMAT, O., FIOLA-MASSON, E., BOUCHARD, A., FAUBERT, D., CHABOT, B. \& COULOMBE, B. 2017. R2TP/Prefoldin-like component RUVBL1/RUVBL2 directly interacts with ZNHIT2 to regulate assembly of U5 small nuclear ribonucleoprotein. Nat Commun, 8, 15615.

COPPOLA, J. A. \& COLE, M. D. 1986. Constitutive c-myc oncogene expression blocks mouse erythroleukaemia cell differentiation but not commitment. Nature, 320, 760-3.

COSTEllo, R. T., MALlet, F., GAUGleR, B., SAINTY, D., ARNOUlET, C., GASTAUT, J. A. \& OLIVE, D. 2000. Human acute myeloid leukemia CD34+/CD38- progenitor cells have decreased sensitivity to chemotherapy and Fas-induced apoptosis, reduced immunogenicity, and impaired dendritic cell transformation capacities. Cancer Res, 60, 4403-11.

CRUK. 2014. Acute myeloid leukaemia (AML) statistics [Online]. Cancer Research UK. Available: http://www.cancerresearchuk.org/cancerinfo/cancerstats/types/leukaemia-aml/ [Accessed 08 February 2015.

DANG, C. V. 2012. MYC on the path to cancer. Cell, 149, 22-35.

DANI, C., BLANCHARD, J. M., PIECHACZYK, M., EL SABOUTY, S., MARTY, L. \& JEANTEUR, P. 1984. Extreme instability of myc mRNA in normal and transformed human cells. Proc Natl Acad Sci U S A, 81, 7046-50.

DARNELL, J. E., JR. 2002. Transcription factors as targets for cancer therapy. Nat Rev Cancer, 2, 740-9. 
DAVIS, A. C., WIMS, M., SPOTTS, G. D., HANN, S. R. \& BRADLEY, A. 1993. A null c-myc mutation causes lethality before 10.5 days of gestation in homozygotes and reduced fertility in heterozygous female mice. Genes Dev, 7, 671-82.

DE BOER, J., WALF-VORDERWULBECKE, V. \& WILLIAMS, O. 2013. In focus: MLL-rearranged leukemia. Leukemia, 27, 1224-8.

DE JONGE, H. J., WOOLTHUIS, C. M., VOS, A. Z., MULDER, A., VAN DEN BERG, E., KLUIN, P. M., VAN DER WEIDE, K., DE BONT, E. S., HULS, G., VELLENGA, E. \& SCHURINGA, J. J. 2011. Gene expression profiling in the leukemic stem cell-enriched CD34+ fraction identifies target genes that predict prognosis in normal karyotype AML. Leukemia, 25, 1825-33.

DELGADO, M. D., ALBAJAR, M., GOMEZ-CASARES, M. T., BATLLE, A. \& LEON, J. 2013. MYC oncogene in myeloid neoplasias. Clin Transl Oncol, $15,87-94$.

DELGADO, M. D., LERGA, A., CANELLES, M., GOMEZ-CASARES, M. T. \& LEON, J. 1995. Differential regulation of Max and role of c-Myc during erythroid and myelomonocytic differentiation of K562 cells. Oncogene, 10, 1659-65.

DIOP, S. B., BERTAUX, K., VASANTHI, D., SARKESHIK, A., GOIRAND, B., ARAGNOL, D., TOLWINSKI, N. S., COLE, M. D., PRADEL, J., YATES, J. R., 3RD, MISHRA, R. K., GRABA, Y. \& SAURIN, A. J. 2008. Reptin and Pontin function antagonistically with $\mathrm{PcG}$ and TrxG complexes to mediate Hox gene control. EMBO Rep, 9, 260-6.

DMITROVSKY, E., KUEHL, W. M., HOLLIS, G. F., KIRSCH, I. R., BENDER, T. P. \& SEGAL, S. 1986. Expression of a transfected human c-myc oncogene inhibits differentiation of a mouse erythroleukaemia cell line. Nature, 322, 748-50.

DOBIN, A., DAVIS, C. A., SCHLESINGER, F., DRENKOW, J., ZALESKI, C., JHA, S., BATUT, P., CHAISSON, M. \& GINGERAS, T. R. 2013. STAR: ultrafast universal RNA-seq aligner. Bioinformatics, 29, 15-21.

DOU, Y., MILNE, T. A., TACKETT, A. J., SMITH, E. R., FUKUDA, A., WYSOCKA, J., ALLIS, C. D., CHAIT, B. T., HESS, J. L. \& ROEDER, R. G. 2005. Physical association and coordinate function of the $\mathrm{H} 3 \mathrm{~K} 4$ methyltransferase MLL1 and the H4 K16 acetyltransferase MOF. Cell, 121, 873-85.

DOULATOV, S., NOTTA, F., LAURENTI, E. \& DICK, J. E. 2012. Hematopoiesis: a human perspective. Cell Stem Cell, 10, 120-36.

DOWNING, J. R. \& SHANNON, K. M. 2002. Acute leukemia: a pediatric perspective. Cancer Cell, 2, 437-45.

DOYON, Y., SELLECK, W., LANE, W. S., TAN, S. \& COTE, J. 2004. Structural and functional conservation of the NuA4 histone acetyltransferase complex from yeast to humans. Mol Cell Biol, 24, 1884-96.

DRABSCH, Y., HUGO, H., ZHANG, R., DOWHAN, D. H., MIAO, Y. R., GEWIRTZ, A. M., BARRY, S. C., RAMSAY, R. G. \& GONDA, T. J. 2007. Mechanism of and requirement for estrogen-regulated MYB expression in estrogen-receptor-positive breast cancer cells. Proc Natl Acad Sci U S A, 104, 13762-7. 
DUGAN, K. A., WOOD, M. A. \& COLE, M. D. 2002. TIP49, but not TRRAP, modulates c-Myc and E2F1 dependent apoptosis. Oncogene, 21, 5835-43.

EISSENBERG, J. C. \& SHILATIFARD, A. 2010. Histone H3 lysine 4 (H3K4) methylation in development and differentiation. Dev Biol, 339, 240-9.

ELKAIM, J., LAMBLIN, M., LAGUERRE, M., ROSENBAUM, J., LESTIENNE, P., ELOY, L., CRESTEIL, T., FELPIN, F. X. \& DESSOLIN, J. 2014. Design, synthesis and biological evaluation of Pontin ATPase inhibitors through a molecular docking approach. Bioorg Med Chem Lett, 24, 2512-6.

EMAMBOKUS, N., VEGIOPOULOS, A., HARMAN, B., JENKINSON, E., ANDERSON, G. \& FRAMPTON, J. 2003. Progression through key stages of haemopoiesis is dependent on distinct threshold levels of c-Myb. Embo j, 22, 4478-88.

EPPERT, K., TAKENAKA, K., LECHMAN, E. R., WALDRON, L., NILSSON, B., VAN GALEN, P., METZELER, K. H., POEPPL, A., LING, V., BEYENE, J., CANTY, A. J., DANSKA, J. S., BOHLANDER, S. K., BUSKE, C., MINDEN, M. D., GOLUB, T. R., JURISICA, I., EBERT, B. L. \& DICK, J. E. 2011. Stem cell gene expression programs influence clinical outcome in human leukemia. Nat Med, 17, 1086-93.

ESTEY, E. \& DOHNER, H. 2006. Acute myeloid leukaemia. Lancet, 368, 1894-907.

ETARD, C., GRADL, D., KUNZ, M., EILERS, M. \& WEDLICH, D. 2005. Pontin and Reptin regulate cell proliferation in early Xenopus embryos in collaboration with c-Myc and Miz-1. Mech Dev, 122, 545-56.

FABER, J., KRIVTSOV, A. V., STUBBS, M. C., WRIGHT, R., DAVIS, T. N., VAN DEN HEUVEL-EIBRINK, M., ZWAAN, C. M., KUNG, A. L. \& ARMSTRONG, S. A. 2009. HOXA9 is required for survival in human MLLrearranged acute leukemias. Blood, 113, 2375-85.

FALANTES, J. F., TRUJILLO, P., PIRUAT, J. I., CALDERON, C., MARQUEZMALAVER, F. J., MARTIN-ANTONIO, B., MILLAN, A., GOMEZ, M., GONZALEZ, J., MARTINO, M. L., MONTERO, I., PARODY, R., ESPIGADO, I., URBANO-ISPIZUA, A. \& PEREZ-SIMON, J. A. 2015. Overexpression of GYS1, MIF, and MYC is associated with adverse outcome and poor response to azacitidine in myelodysplastic syndromes and acute myeloid leukemia. Clin Lymphoma Myeloma Leuk, 15, 236-44.

FAN, J., ZELLER, K., CHEN, Y. C., WATKINS, T., BARNES, K. C., BECKER, K. G., DANG, C. V. \& CHEADLE, C. 2010. Time-dependent c-Myc transactomes mapped by Array-based nuclear run-on reveal transcriptional modules in human B cells. PLoS One, 5, e9691.

FAULK, K., GORE, L. \& COOPER, T. 2014. Overview of therapy and strategies for optimizing outcomes in de novo pediatric acute myeloid leukemia. Paediatr Drugs, 16, 213-27.

FENG, Y., LEE, N. \& FEARON, E. R. 2003. TIP49 regulates beta-catenin-mediated neoplastic transformation and $\mathrm{T}$-cell factor target gene induction via effects on chromatin remodeling. Cancer Res, 63, 8726-34.

FERRANDO, A. A., ARMSTRONG, S. A., NEUBERG, D. S., SALLAN, S. E., SILVERMAN, L. B., KORSMEYER, S. J. \& LOOK, A. T. 2003. Gene expression signatures in MLL-rearranged T-lineage and B-precursor acute leukemias: dominance of HOX dysregulation. Blood, 102, 262-8. 
FRANK, S. R., PARISI, T., TAUBERT, S., FERNANDEZ, P., FUCHS, M., CHAN, H. M., LIVINGSTON, D. M. \& AMATI, B. 2003. MYC recruits the TIP60 histone acetyltransferase complex to chromatin. EMBO Rep, 4, 575-80.

FUCHS, M., GERBER, J., DRAPKIN, R., SIF, S., IKURA, T., OGRYZKO, V., LANE, W. S., NAKATANI, Y. \& LIVINGSTON, D. M. 2001. The p400 complex is an essential E1A transformation target. Cell, 106, 297-307.

GALLANT, P. 2007. Control of transcription by Pontin and Reptin. Trends Cell Biol, 17, 187-92.

GEWIRTZ, A. M. \& CALABRETTA, B. 1988. A c-myb antisense oligodeoxynucleotide inhibits normal human hematopoiesis in vitro. Science, 242, 1303-6.

GNANAPRAKASAM, J. N. \& WANG, R. 2017. MYC in Regulating Immunity: Metabolism and Beyond. Genes (Basel), 8.

GONDA, T. J., BUCKMASTER, C. \& RAMSAY, R. G. 1989. Activation of c-myb by carboxy-terminal truncation: relationship to transformation of murine haemopoietic cells in vitro. Embo j, 8, 1777-83.

GONDA, T. J. \& METCALF, D. 1984. Expression of myb, myc and fos protooncogenes during the differentiation of a murine myeloid leukaemia. Nature, 310, 249-51.

GORYNIA, S., BANDEIRAS, T. M., PINHO, F. G., MCVEY, C. E., VONRHEIN, C., ROUND, A., SVERGUN, D. I., DONNER, P., MATIAS, P. M. \& CARRONDO, M. A. 2011. Structural and functional insights into a dodecameric molecular machine - the RuvBL1/RuvBL2 complex. J Struct Biol, 176, 279-91.

GREIG, K. T., CAROTTA, S. \& NUTT, S. L. 2008. Critical roles for c-Myb in hematopoietic progenitor cells. Semin Immunol, 20, 247-56.

GRIBUN, A., CHEUNG, K. L., HUEN, J., ORTEGA, J. \& HOURY, W. A. 2008. Yeast Rvb1 and Rvb2 are ATP-dependent DNA helicases that form a heterohexameric complex. J Mol Biol, 376, 1320-33.

GRIEB, B. C., BOYD, K., MITRA, R. \& EISCHEN, C. M. 2016. Haploinsufficiency of the Myc regulator Mtbp extends survival and delays tumor development in aging mice. Aging (Albany NY), 8, 2590-2602.

GRIEB, B. C., GRAMLING, M. W., ARRATE, M. P., CHEN, X., BEAUPARLANT, S. L., HAINES, D. S., XIAO, H. \& EISCHEN, C. M. 2014. Oncogenic protein MTBP interacts with MYC to promote tumorigenesis. Cancer Res, 74, 3591-602.

GRIGOLETTO, A., LESTIENNE, P. \& ROSENBAUM, J. 2011. The multifaceted proteins Reptin and Pontin as major players in cancer. Biochim Biophys Acta, $1815,147-57$.

GRIGOLETTO, A., NEAUD, V., ALLAIN-COURTOIS, N., LESTIENNE, P. \& ROSENBAUM, J. 2013. The ATPase activity of reptin is required for its effects on tumor cell growth and viability in hepatocellular carcinoma. Mol Cancer Res, 11, 133-9.

GRIMWADE, D., HILLS, R. K., MOORMAN, A. V., WALKER, H., CHATTERS, S., GOLDSTONE, A. H., WHEATLEY, K., HARRISON, C. J. \& BURNETT, A. K. 2010. Refinement of cytogenetic classification in acute myeloid leukemia: determination of prognostic significance of rare recurring 
chromosomal abnormalities among 5876 younger adult patients treated in the United Kingdom Medical Research Council trials. Blood, 116, 354-65.

GRUBER, T., \& DOWNING, J. 2012. Molecular genetics of acute myeloid leukemia. In: CHING-HON PUI, S. J. S. C. S. R. H. (ed.) Childhood Leukemias. 3rd ed. Cambridge: Cambridge University Press.

GUERIN, M., SHENG, Z. M., ANDRIEU, N. \& RIOU, G. 1990. Strong association between c-myb and oestrogen-receptor expression in human breast cancer. Oncogene, 5, 131-5.

GUO, Y., NIU, C., BRESLIN, P., TANG, M., ZHANG, S., WEI, W., KINI, A. R., PANER, G. P., ALKAN, S., MORRIS, S. W., DIAZ, M., STIFF, P. J. \& ZHANG, J. 2009. c-Myc-mediated control of cell fate in megakaryocyteerythrocyte progenitors. Blood, 114, 2097-106.

HANEKAMP, D., CLOOS, J. \& SCHUURHUIS, G. J. 2017. Leukemic stem cells: identification and clinical application. Int J Hematol, 105, 549-557.

HANN, S. R. \& EISENMAN, R. N. 1984. Proteins encoded by the human c-myc oncogene: differential expression in neoplastic cells. Mol Cell Biol, 4, 248697.

HANN, S. R., THOMPSON, C. B. \& EISENMAN, R. N. 1985. c-myc oncogene protein synthesis is independent of the cell cycle in human and avian cells. Nature, 314, 366-9.

HARRISON, C. J., HILLS, R. K., MOORMAN, A. V., GRIMWADE, D. J., HANN, I., WEBB, D. K., WHEATLEY, K., DE GRAAF, S. S., VAN DEN BERG, E., BURNETT, A. K. \& GIBSON, B. E. 2010. Cytogenetics of childhood acute myeloid leukemia: United Kingdom Medical Research Council Treatment trials AML 10 and 12. J Clin Oncol, 28, 2674-81.

HAURIE, V., MENARD, L., NICOU, A., TOURIOL, C., METZLER, P., FERNANDEZ, J., TARAS, D., LESTIENNE, P., BALABAUD, C., BIOULAC-SAGE, P., PRATS, H., ZUCMAN-ROSSI, J. \& ROSENBAUM, J. 2009. Adenosine triphosphatase pontin is overexpressed in hepatocellular carcinoma and coregulated with reptin through a new posttranslational mechanism. Hepatology, 50, 1871-83.

HESS, J. L. 2004. MLL, Hox genes, and leukemia: the plot thickens. Blood, 103, 2870-2871.

HESS, J. L., BITTNER, C. B., ZEISIG, D. T., BACH, C., FUCHS, U., BORKHARDT, A., FRAMPTON, J. \& SLANY, R. K. 2006. c-Myb is an essential downstream target for homeobox-mediated transformation of hematopoietic cells. Blood, 108, 297-304.

HOLZMANN, K., GERNER, C., KOROSEC, T., POLTL, A., GRIMM, R. \& SAUERMANN, G. 1998. Identification and characterization of the ubiquitously occurring nuclear matrix protein NMP 238. Biochem Biophys Res Commun, 252, 39-45.

HORTON, S. J., GRIER, D. G., MCGONIGLE, G. J., THOMPSON, A., MORROW, M., DE SILVA, I., MOULDING, D. A., KIOUSSIS, D., LAPPIN, T. R., BRADY, H. J. \& WILLIAMS, O. 2005. Continuous MLLENL expression is necessary to establish a "Hox Code" and maintain immortalization of hematopoietic progenitor cells. Cancer Res, 65, 9245-52.

HORTON, S. J. \& HUNTLY, B. J. 2012. Recent advances in acute myeloid leukemia stem cell biology. Haematologica, 97, 966-74. 
HOSOKAWA, H., TANAKA, T., KATO, M., SHINODA, K., TOHYAMA, H., HANAZAWA, A., TAMAKI, Y., HIRAHARA, K., YAGI, R., SAKIKAWA, I., MORITA, A., NAGIRA, M., POYUROVSKY, M. V., SUZUKI, Y., MOTOHASHI, S. \& NAKAYAMA, T. 2013. Gata3/Ruvbl2 complex regulates $\mathrm{T}$ helper 2 cell proliferation via repression of $\mathrm{Cdkn} 2 \mathrm{c}$ expression. Proc Natl Acad Sci U S A, 110, 18626-31.

HSIEH, J. J., CHENG, E. H. \& KORSMEYER, S. J. 2003a. Taspase1: a threonine aspartase required for cleavage of MLL and proper HOX gene expression. Cell, 115, 293-303.

HSIEH, J. J., ERNST, P., ERDJUMENT-BROMAGE, H., TEMPST, P. \& KORSMEYER, S. J. 2003b. Proteolytic cleavage of MLL generates a complex of $\mathrm{N}$ - and C-terminal fragments that confers protein stability and subnuclear localization. Mol Cell Biol, 23, 186-94.

HTTP://PANTHERDB.ORG. Protein ANalysis THrough Evolutionary Relationships [Online].

HUANG, X., SPENCER, G. J., LYNCH, J. T., CICERI, F., SOMERVILLE, T. D. \& SOMERVAILLE, T. C. 2014. Enhancers of Polycomb EPC1 and EPC2 sustain the oncogenic potential of MLL leukemia stem cells. Leukemia, 28, 1081-91.

HUBER, O., MENARD, L., HAURIE, V., NICOU, A., TARAS, D. \& ROSENBAUM, J. 2008. Pontin and reptin, two related ATPases with multiple roles in cancer. Cancer Res, 68, 6873-6.

HUEN, J., KAKIHARA, Y., UGWU, F., CHEUNG, K. L., ORTEGA, J. \& HOURY, W. A. 2010. Rvb1-Rvb2: essential ATP-dependent helicases for critical complexes. Biochem Cell Biol, 88, 29-40.

HUNTLY, B. J. \& GILLILAND, D. G. 2005. Leukaemia stem cells and the evolution of cancer-stem-cell research. Nat Rev Cancer, 5, 311-21.

IIZUKA, N., TSUNEDOMI, R., TAMESA, T., OKADA, T., SAKAMOTO, K., HAMAGUCHI, T., YAMADA-OKABE, H., MIYAMOTO, T., UCHIMURA, S., HAMAMOTO, Y. \& OKA, M. 2006. Involvement of cmyc-regulated genes in hepatocellular carcinoma related to genotype-C hepatitis B virus. J Cancer Res Clin Oncol, 132, 473-81.

IKURA, T., OGRYZKO, V. V., GRIGORIEV, M., GROISMAN, R., WANG, J., HORIKOSHI, M., SCULLY, R., QIN, J. \& NAKATANI, Y. 2000. Involvement of the TIP60 histone acetylase complex in DNA repair and apoptosis. Cell, 102, 463-73.

ILLUMINA. 2014. The RNA Express App user guide. Available: https://support.illumina.com/content/dam/illuminasupport/documents/documentation/software_documentation/basespace/rnaexpress-user-guide-15052918a.pdf [Accessed 16/05/2017].

IRITANI, B. M. \& EISENMAN, R. N. 1999. c-Myc enhances protein synthesis and cell size during B lymphocyte development. Proc Natl Acad Sci U S A, 96, 13180-5.

IWASAKI, H. \& AKASHI, K. 2007a. Hematopoietic developmental pathways: on cellular basis. Oncogene, 26, 6687-96.

IWASAKI, H. \& AKASHI, K. 2007b. Myeloid lineage commitment from the hematopoietic stem cell. Immunity, 26, 726-40. 
IZUMI, N., YAMASHITA, A., HIRANO, H. \& OHNO, S. 2012. Heat shock protein 90 regulates phosphatidylinositol 3-kinase-related protein kinase family proteins together with the RUVBL1/2 and Tel2-containing co-factor complex. Cancer Sci, 103, 50-7.

IZUMI, N., YAMASHITA, A., IWAMATSU, A., KURATA, R., NAKAMURA, H., SAARI, B., HIRANO, H., ANDERSON, P. \& OHNO, S. 2010. AAA+ proteins RUVBL1 and RUVBL2 coordinate PIKK activity and function in nonsense-mediated mRNA decay. Sci Signal, 3, ra27.

JAGANNATHAN-BOGDAN, M. \& ZON, L. I. 2013. Hematopoiesis. Development, 140, 2463-7.

JHA, S. \& DUTTA, A. 2009. RVB1/RVB2: running rings around molecular biology. Mol Cell, 34, 521-33.

JHA, S., SHIBATA, E. \& DUTTA, A. 2008. Human Rvb1/Tip49 is required for the histone acetyltransferase activity of Tip60/NuA4 and for the downregulation of phosphorylation on H2AX after DNA damage. Mol Cell Biol, 28, 2690700 .

JIN, J., CAI, Y., YAO, T., GOTTSCHALK, A. J., FLORENS, L., SWANSON, S. K., GUTIERREZ, J. L., COLEMAN, M. K., WORKMAN, J. L., MUSHEGIAN, A., WASHBURN, M. P., CONAWAY, R. C. \& CONAWAY, J. W. 2005. A mammalian chromatin remodeling complex with similarities to the yeast INO80 complex. J Biol Chem, 280, 41207-12.

JIN, S., ZHAO, H., YI, Y., NAKATA, Y., KALOTA, A. \& GEWIRTZ, A. M. 2010. c-Myb binds MLL through menin in human leukemia cells and is an important driver of MLL-associated leukemogenesis. J Clin Invest, 120, 593606.

JOHNSTON, L. A., PROBER, D. A., EDGAR, B. A., EISENMAN, R. N. \& GALLANT, P. 1999. Drosophila myc regulates cellular growth during development. Cell, 98, 779-90.

JONSSON, Z. O., JHA, S., WOHLSCHLEGEL, J. A. \& DUTTA, A. 2004. Rvb1p/Rvb2p recruit Arp5p and assemble a functional Ino80 chromatin remodeling complex. Mol Cell, 16, 465-77.

KANEMAKI, M., KUROKAWA, Y., MATSU-URA, T., MAKINO, Y., MASANI, A., OKAZAKI, K., MORISHITA, T. \& TAMURA, T. A. 1999. TIP49b, a new RuvB-like DNA helicase, is included in a complex together with another RuvB-like DNA helicase, TIP49a. J Biol Chem, 274, 22437-44.

KANEMAKI, M., MAKINO, Y., YOSHIDA, T., KISHIMOTO, T., KOGA, A., YAMAMOTO, K., YAMAMOTO, M., MONCOLLIN, V., EGLY, J. M., MURAMATSU, M. \& TAMURA, T. 1997. Molecular cloning of a rat 49$\mathrm{kDa}$ TBP-interacting protein (TIP49) that is highly homologous to the bacterial RuvB. Biochem Biophys Res Commun, 235, 64-8.

KAWAGOE, H., KANDILCI, A., KRANENBURG, T. A. \& GROSVELD, G. C. 2007. Overexpression of N-Myc rapidly causes acute myeloid leukemia in mice. Cancer Res, 67, 10677-85.

KIM, J. H., CHOI, H. J., KIM, B., KIM, M. H., LEE, J. M., KIM, I. S., LEE, M. H., CHOI, S. J., KIM, K. I., KIM, S. I., CHUNG, C. H. \& BAEK, S. H. 2006a. Roles of sumoylation of a reptin chromatin-remodelling complex in cancer metastasis. Nat Cell Biol, 8, 631-9. 
KIM, J. H., KIM, B., CAI, L., CHOI, H. J., OHGI, K. A., TRAN, C., CHEN, C., CHUNG, C. H., HUBER, O., ROSE, D. W., SAWYERS, C. L., ROSENFELD, M. G. \& BAEK, S. H. 2005. Transcriptional regulation of a metastasis suppressor gene by Tip60 and beta-catenin complexes. Nature, 434, 921-6.

KIM, J. H., LEE, J. M., NAM, H. J., CHOI, H. J., YANG, J. W., LEE, J. S., KIM, M. H., KIM, S. I., CHUNG, C. H., KIM, K. I. \& BAEK, S. H. 2007. SUMOylation of pontin chromatin-remodeling complex reveals a signal integration code in prostate cancer cells. Proc Natl Acad Sci U S A, 104, 20793-8.

KIM, Y. H., GIRARD, L., GIACOMINI, C. P., WANG, P., HERNANDEZBOUSSARD, T., TIBSHIRANI, R., MINNA, J. D. \& POLLACK, J. R. 2006b. Combined microarray analysis of small cell lung cancer reveals altered apoptotic balance and distinct expression signatures of MYC family gene amplification. Oncogene, 25, 130-8.

KRIVTSOV, A. V. \& ARMSTRONG, S. A. 2007. MLL translocations, histone modifications and leukaemia stem-cell development. Nat Rev Cancer, 7, 82333.

KRIVTSOV, A. V., TWOMEY, D., FENG, Z., STUBBS, M. C., WANG, Y., FABER, J., LEVINE, J. E., WANG, J., HAHN, W. C., GILLILAND, D. G., GOLUB, T. R. \& ARMSTRONG, S. A. 2006. Transformation from committed progenitor to leukaemia stem cell initiated by MLL-AF9. Nature, 442, 818-22.

KVINLAUG, B. T., CHAN, W. I., BULLINGER, L., RAMASWAMI, M., SEARS, C., FOSTER, D., LAZIC, S. E., OKABE, R., BENNER, A., LEE, B. H., DE SILVA, I., VALK, P. J., DELWEL, R., ARMSTRONG, S. A., DOHNER, H., GILLILAND, D. G. \& HUNTLY, B. J. 2011. Common and overlapping oncogenic pathways contribute to the evolution of acute myeloid leukemias. Cancer Res, 71, 4117-29.

LAHORTIGA, I., DE KEERSMAECKER, K., VAN VLIERBERGHE, P., GRAUX, C., CAUWELIER, B., LAMBERT, F., MENTENS, N., BEVERLOO, H. B., PIETERS, R., SPELEMAN, F., ODERO, M. D., BAUTERS, M., FROYEN, G., MARYNEN, P., VANDENBERGHE, P., WLODARSKA, I., MEIJERINK, J. P. \& COOLS, J. 2007. Duplication of the MYB oncogene in T cell acute lymphoblastic leukemia. Nat Genet, 39, 593-5.

LAMB, J., CRAWFORD, E. D., PECK, D., MODELL, J. W., BLAT, I. C., WROBEL, M. J., LERNER, J., BRUNET, J. P., SUBRAMANIAN, A., ROSS, K. N., REICH, M., HIERONYMUS, H., WEI, G., ARMSTRONG, S. A., HAGgarty, S. J., ClEMONS, P. A., WEI, R., CARR, S. A., LANDER, E. S. \& GOLUB, T. R. 2006. The Connectivity Map: using geneexpression signatures to connect small molecules, genes, and disease. Science, 313, 1929-35.

LAPIDOT, T., SIRARD, C., VORMOOR, J., MURDOCH, B., HOANG, T., CACERES-CORTES, J., MINDEN, M., PATERSON, B., CALIGIURI, M. A. \& DICK, J. E. 1994. A cell initiating human acute myeloid leukaemia after transplantation into SCID mice. Nature, 367, 645-8.

LARSSON, L. G., IVHED, I., GIDLUND, M., PETTERSSON, U., VENNSTROM, B. \& NILSSON, K. 1988. Phorbol ester-induced terminal differentiation is 
inhibited in human U-937 monoblastic cells expressing a v-myc oncogene. Proc Natl Acad Sci U S A, 85, 2638-42.

LAUSCHER, J. C., LODDENKEMPER, C., KOSEL, L., GRONE, J., BUHR, H. J. \& HUBER, O. 2007. Increased pontin expression in human colorectal cancer tissue. Hum Pathol, 38, 978-85.

LEE, J. S., KIM, Y., KIM, I. S., KIM, B., CHOI, H. J., LEE, J. M., SHIN, H. J., KIM, J. H., KIM, J. Y., SEO, S. B., LEE, H., BINDA, O., GOZANI, O., SEMENZA, G. L., KIM, M., KIM, K. I., HWANG, D. \& BAEK, S. H. 2010. Negative regulation of hypoxic responses via induced Reptin methylation. Mol Cell, 39, 71-85.

LI, W., ZENG, J., LI, Q., ZHAO, L., LIU, T., BJORKHOLM, M., JIA, J. \& XU, D. 2010. Reptin is required for the transcription of telomerase reverse transcriptase and over-expressed in gastric cancer. Mol Cancer, 9, 132.

LIEU, Y. K. \& REDDY, E. P. 2009. Conditional c-myb knockout in adult hematopoietic stem cells leads to loss of self-renewal due to impaired proliferation and accelerated differentiation. Proc Natl Acad Sci U S A, 106, 21689-94.

LIN, C., SMITH, E. R., TAKAHASHI, H., LAI, K. C., MARTIN-BROWN, S., FLORENS, L., WASHBURN, M. P., CONAWAY, J. W., CONAWAY, R. C. \& SHILATIFARD, A. 2010. AFF4, a component of the ELL/P-TEFb elongation complex and a shared subunit of MLL chimeras, can link transcription elongation to leukemia. Mol Cell, 37, 429-37.

LIN, C. Y., LOVEN, J., RAHL, P. B., PARANAL, R. M., BURGE, C. B., BRADNER, J. E., LEE, T. I. \& YOUNG, R. A. 2012. Transcriptional amplification in tumor cells with elevated c-Myc. Cell, 151, 56-67.

LIYANAGE, S. U., COYAUD, E., LAURENT, E. M., HURREN, R., MACLEAN, N., WOOD, S. R., KAZAK, L., SHAMAS-DIN, A., HOLT, I., RAUGHT, B. \& SCHIMMER, A. 2017. Characterizing the mitochondrial DNA polymerase gamma interactome by BioID identifies Ruvbl2 localizes to the mitochondria. Mitochondrion, 32, 31-35.

LOPEZ-PERROTE, A., MUNOZ-HERNANDEZ, H., GIL, D. \& LLORCA, O. 2012. Conformational transitions regulate the exposure of a DNA-binding domain in the RuvBL1-RuvBL2 complex. Nucleic Acids Res, 40, 11086-99.

LOWENBERG, B., DOWNING, J. R. \& BURNETT, A. 1999. Acute myeloid leukemia. $N$ Engl J Med, 341, 1051-62.

LUO, H., LI, Q., O'NEAL, J., KREISEL, F., LE BEAU, M. M. \& TOMASSON, M.

H. 2005. c-Myc rapidly induces acute myeloid leukemia in mice without evidence of lymphoma-associated antiapoptotic mutations. Blood, 106, 245261.

LUSCHER, B. \& LARSSON, L. G. 1999. The basic region/helix-loop-helix/leucine zipper domain of Myc proto-oncoproteins: function and regulation. Oncogene, 18, 2955-66.

MAJETI, R., CHAO, M. P., ALIZADEH, A. A., PANG, W. W., JAISWAL, S., GIBBS, K. D., JR., VAN ROOIJEN, N. \& WEISSMAN, I. L. 2009. CD47 is an adverse prognostic factor and therapeutic antibody target on human acute myeloid leukemia stem cells. Cell, 138, 286-99. 
MAKINO, Y., KANEMAKI, M., KUROKAWA, Y., KOJI, T. \& TAMURA, T. 1999. A rat RuvB-like protein, TIP49a, is a germ cell-enriched novel DNA helicase. J Biol Chem, 274, 15329-35.

MANSOUR, M. R., ABRAHAM, B. J., ANDERS, L., BEREZOVSKAYA, A., GUTIERREZ, A., DURBIN, A. D., ETCHIN, J., LAWTON, L., SALLAN, S. E., SILVERMAN, L. B., LOH, M. L., HUNGER, S. P., SANDA, T., YOUNG, R. A. \& LOOK, A. T. 2014. Oncogene regulation. An oncogenic super-enhancer formed through somatic mutation of a noncoding intergenic element. Science, 346, 1373-7.

MAO, Y. Q. \& HOURY, W. A. 2017. The Role of Pontin and Reptin in Cellular Physiology and Cancer Etiology. Front Mol Biosci, 4, 58.

MARTINEZ, P. \& BLASCO, M. A. 2017. Telomere-driven diseases and telomeretargeting therapies. J Cell Biol, 216, 875-887.

MASLON, M. M., HRSTKA, R., VOJTESEK, B. \& HUPP, T. R. 2010. A divergent substrate-binding loop within the pro-oncogenic protein anterior gradient-2 forms a docking site for Reptin. J Mol Biol, 404, 418-38.

MATIAS, P. M., GORYNIA, S., DONNER, P. \& CARRONDO, M. A. 2006. Crystal structure of the human AAA+ protein RuvBL1. J Biol Chem, 281, 38918-29.

MCCUBREY, J. A., STEELMAN, L. S., BERTRAND, F. E., DAVIS, N. M., ABRAMS, S. L., MONTALTO, G., D'ASSORO, A. B., LIBRA, M., NICOLETTI, F., MAESTRO, R., BASECKE, J., COCCO, L., CERVELLO, M. \& MARTELLI, A. M. 2014. Multifaceted roles of GSK-3 and Wnt/betacatenin in hematopoiesis and leukemogenesis: opportunities for therapeutic intervention. Leukemia, 28, 15-33.

MCDERMOTT, S. P., EPPERT, K., LECHMAN, E. R., DOEDENS, M. \& DICK, J. E. 2010. Comparison of human cord blood engraftment between immunocompromised mouse strains. Blood, 116, 193-200.

MCKEEGAN, K. S., DEBIEUX, C. M. \& WATKINS, N. J. 2009. Evidence that the AAA+ proteins TIP48 and TIP49 bridge interactions between $15.5 \mathrm{~K}$ and the related NOP56 and NOP58 proteins during box C/D snoRNP biogenesis. Mol Cell Biol, 29, 4971-81.

MCMAHON, S. B., WOOD, M. A. \& COLE, M. D. 2000. The essential cofactor TRRAP recruits the histone acetyltransferase hGCN5 to c-Myc. Mol Cell Biol, 20, 556-62.

MENARD, L., TARAS, D., GRIGOLETTO, A., HAURIE, V., NICOU, A., DUGOT-SENANT, N., COSTET, P., ROUSSEAU, B. \& ROSENBAUM, J. 2010. In vivo silencing of Reptin blocks the progression of human hepatocellular carcinoma in xenografts and is associated with replicative senescence. J Hepatol, 52, 681-9.

MENSSEN, A. \& HERMEKING, H. 2002. Characterization of the c-MYC-regulated transcriptome by SAGE: identification and analysis of c-MYC target genes. Proc Natl Acad Sci U S A, 99, 6274-9.

METCALF, D., CARPINELli, M. R., HYLAND, C., MIFSUD, S., DIRAGO, L., NICOLA, N. A., HILTON, D. J. \& ALEXANDER, W. S. 2005. Anomalous megakaryocytopoiesis in mice with mutations in the c-Myb gene. Blood, 105, 3480-7. 
MEYER, C., HOFMANN, J., BURMEISTER, T., GROGER, D., PARK, T. S., EMERENCIANO, M., POMBO DE OLIVEIRA, M., RENNEVILLE, A., VILLARESE, P., MACINTYRE, E., CAVE, H., CLAPPIER, E., MASSMALO, K., ZUNA, J., TRKA, J., DE BRAEKELEER, E., DE BRAEKELEER, M., OH, S. H., TSAUR, G., FECHINA, L., VAN DER VELDEN, V. H., VAN DONGEN, J. J., DELABESSE, E., BINATO, R., SILVA, M. L., KUSTANOVICH, A., ALEINIKOVA, O., HARRIS, M. H., LUND-AHO, T., JUVONEN, V., HEIDENREICH, O., VORMOOR, J., CHOI, W. W., JAROSOVA, M., KOLENOVA, A., BUENO, C., MENENDEZ, P., WEHNER, S., ECKERT, C., TALMANT, P., TONDEUR, S., LIPPERT, E., LAUNAY, E., HENRY, C., BALLERINI, P., LAPILLONE, H., CALLANAN, M. B., CAYUELA, J. M., HERBAUX, C., CAZZANIGA, G., KAKADIYA, P. M., BOHLANDER, S., AHLMANN, M., CHOI, J. R., GAMEIRO, P., LEE, D. S., KRAUTER, J., CORNILLETLEFEBVRE, P., TE KRONNIE, G., SCHAFER, B. W., KUBETZKO, S., ALONSO, C. N., ZUR STADT, U., SUTTON, R., VENN, N. C., IZRAELI, S., TRAKHTENBROT, L., MADSEN, H. O., ARCHER, P., HANCOCK, J., CERVEIRA, N., TEIXEIRA, M. R., LO NIGRO, L., MORICKE, A., STANULLA, M., SCHRAPPE, M., SEDEK, L., SZCZEPANSKI, T., ZWAAN, C. M., COENEN, E. A., VAN DEN HEUVEL-EIBRINK, M. M., STREHL, S., DWORZAK, M., PANZER-GRUMAYER, R., DINGERMANN, T., KLINGEBIEL, T. \& MARSCHALEK, R. 2013. The MLL recombinome of acute leukemias in 2013. Leukemia, 27, 2165-76.

MÉZARD, C., DAVIES, A. A., STASIAK, A. \& WEST, S. C. 1997. Biochemical properties of RuvBD113N: a mutation in helicase motif II of the RuvB hexamer affects DNA binding and ATPase activities1. Journal of Molecular Biology, 271, 704-717.

MI, H., HUANG, X., MURUGANUJAN, A., TANG, H., MILLS, C., KANG, D. \& THOMAS, P. D. 2017. PANTHER version 11: expanded annotation data from Gene Ontology and Reactome pathways, and data analysis tool enhancements. Nucleic Acids Res, 45, D183-d189.

MILNE, T. A., BRIGGS, S. D., BROCK, H. W., MARTIN, M. E., GIBBS, D., ALLIS, C. D. \& HESS, J. L. 2002. MLL targets SET domain methyltransferase activity to Hox gene promoters. Mol Cell, 10, 1107-17.

MILONE, M. R., PUCCI, B., COLANGELO, T., LOMBARDI, R., IANNELLI, F., COLANTUONI, V., SABATINO, L. \& BUDILLON, A. 2016. Proteomic characterization of peroxisome proliferator-activated receptor-gamma (PPARgamma) overexpressing or silenced colorectal cancer cells unveils a novel protein network associated with an aggressive phenotype. Mol Oncol, 10, 1344-62.

MORRISON, S. J. \& WEISSMAN, I. L. 1994. The long-term repopulating subset of hematopoietic stem cells is deterministic and isolatable by phenotype. Immunity, 1, 661-73.

MOSCOVICI, C., GAZZOLO, L. \& MOSCOVICI, M. G. 1975. Focus assay and defectiveness of avian myeloblastosis virus. Virology, 68, 173-81.

MROZEK, K., HEEREMA, N. A. \& BLOOMFIELD, C. D. 2004. Cytogenetics in acute leukemia. Blood Rev, 18, 115-36. 
MUCENSKI, M. L., MCLAIN, K., KIER, A. B., SWERDLOW, S. H., SCHREINER, C. M., MILLER, T. A., PIETRYGA, D. W., SCOTT, W. J., JR. \& POTTER, S. S. 1991. A functional c-myb gene is required for normal murine fetal hepatic hematopoiesis. Cell, 65, 677-89.

MUllER-TIDOW, C., STEFFEN, B., CAUVET, T., TICKENBROCK, L., JI, P., DIEDERICHS, S., SARGIN, B., KOHLER, G., STELLJES, M., PUCCETTI, E., RUTHARDT, M., DEVOS, S., HIEBERT, S. W., KOEFFLER, H. P., BERDEL, W. E. \& SERVE, H. 2004. Translocation products in acute myeloid leukemia activate the Wnt signaling pathway in hematopoietic cells. Mol Cell Biol, 24, 2890-904.

MUNTEAN, A. G. \& HESS, J. L. 2012. The pathogenesis of mixed-lineage leukemia. Annu Rev Pathol, 7, 283-301.

MURATI, A., GERVAIS, C., CARBUCCIA, N., FINETTI, P., CERVERA, N., ADELAIDE, J., STRUSKI, S., LIPPERT, E., MUGNERET, F., TIGAUD, I., PENTHER, D., BASTARD, C., POPPE, B., SPELEMAN, F., BARANGER, L., LUQUET, I., CORNILLET-LEFEBVRE, P., NADAL, N., NGUYENKHAC, F., PEROT, C., OLSCHWANG, S., BERTUCCI, F., CHAFFANET, M., LESSARD, M., MOZZICONACCI, M. J. \& BIRNBAUM, D. 2009. Genome profiling of acute myelomonocytic leukemia: alteration of the MYB locus in MYST3-linked cases. Leukemia, 23, 85-94.

MURPHY, M. J., WILSON, A. \& TRUMPP, A. 2005. More than just proliferation: Myc function in stem cells. Trends Cell Biol, 15, 128-37.

NANO, N. \& HOURY, W. A. 2013. Chaperone-like activity of the AAA+ proteins Rvb1 and Rvb2 in the assembly of various complexes. Philos Trans $R$ Soc Lond B Biol Sci, 368, 20110399.

NG, S. W., MITCHELl, A., KENNEDY, J. A., CHEN, W. C., MCLEOD, J., IBRAHIMOVA, N., ARRUDA, A., POPESCU, A., GUPTA, V., SCHIMMER, A. D., SCHUH, A. C., YEE, K. W., BULLINGER, L., HEROLD, T., GORLICH, D., BUCHNER, T., HIDDEMANN, W., BERDEL, W. E., WORMANN, B., CHEOK, M., PREUDHOMME, C., DOMBRET, H., METZELER, K., BUSKE, C., LOWENBERG, B., VALK, P. J., ZANDSTRA, P. W., MINDEN, M. D., DICK, J. E. \& WANG, J. C. 2016. A 17-gene stemness score for rapid determination of risk in acute leukaemia. Nature, 540, 433-437.

NIE, Z., HU, G., WEI, G., CUI, K., YAMANE, A., RESCH, W., WANG, R., GREEN, D. R., TESSAROLlO, L., CASEllAS, R., ZHAO, K. \& LEVENS, D. 2012. c-Myc is a universal amplifier of expressed genes in lymphocytes and embryonic stem cells. Cell, 151, 68-79.

NIEWIAROWSKI, A., BRADLEY, A. S., GOR, J., MCKAY, A. R., PERKINS, S. J. \& TSANEVA, I. R. 2010. Oligomeric assembly and interactions within the human RuvB-like RuvBL1 and RuvBL2 complexes. Biochem J, 429, 113-25.

O'NEIL, J., TCHINDA, J., GUTIERREZ, A., MOREAU, L., MASER, R. S., WONG, K. K., LI, W., MCKENNA, K., LIU, X. S., FENG, B., NEUBERG, D., SILVERMAN, L., DEANGELO, D. J., KUTOK, J. L., ROTHSTEIN, R., DEPINHO, R. A., CHIN, L., LEE, C. \& LOOK, A. T. 2007. Alu elements mediate MYB gene tandem duplication in human T-ALL. J Exp Med, 204, 3059-66. 
OELGESCHLAGER, M., NUCHPRAYOON, I., LUSCHER, B. \& FRIEDMAN, A. D. 1996. C/EBP, c-Myb, and PU.1 cooperate to regulate the neutrophil elastase promoter. Mol Cell Biol, 16, 4717-25.

ORKIN, S. H. 2000. Diversification of haematopoietic stem cells to specific lineages. Nat Rev Genet, 1, 57-64.

ORKIN, S. H. \& ZON, L. I. 2008. Hematopoiesis: an evolving paradigm for stem cell biology. Cell, 132, 631-44.

OSAKI, H. 2011. Investigation of the leukaemic activity of MLLfusions in human haematopoietic cells. $\mathrm{PhD}$, University College London.

OSAKI, H., WALF-VORDERWULBECKE, V., MANGOLINI, M., ZHAO, L., HORTON, S. J., MORRONE, G., SCHURINGA, J. J., DE BOER, J. \& WILLIAMS, O. 2013. The AAA+ ATPase RUVBL2 is a critical mediator of MLL-AF9 oncogenesis. Leukemia, 27, 1461-8.

OSAWA, M., HANADA, K., HAMADA, H. \& NAKAUCHI, H. 1996. Long-term lymphohematopoietic reconstitution by a single CD34-low/negative hematopoietic stem cell. Science, 273, 242-5.

PATTABIRAMAN, D. R. \& GONDA, T. J. 2013. Role and potential for therapeutic targeting of MYB in leukemia. Leukemia, 27, 269-77.

PELENGARIS, S., KHAN, M. \& EVAN, G. 2002. c-MYC: more than just a matter of life and death. Nat Rev Cancer, 2, 764-76.

PINEAULT, N., HELGASON, C. D., LAWRENCE, H. J. \& HUMPHRIES, R. K. 2002. Differential expression of Hox, Meis1, and Pbx 1 genes in primitive cells throughout murine hematopoietic ontogeny. Exp Hematol, 30, 49-57.

POLlYEA, D. A., GUTMAN, J. A., GORE, L., SMITH, C. A. \& JORDAN, C. T. 2014. Targeting acute myeloid leukemia stem cells: a review and principles for the development of clinical trials. Haematologica, 99, 1277-84.

POOLE, C. J. \& VAN RIGGELEN, J. 2017. MYC-Master Regulator of the Cancer Epigenome and Transcriptome. Genes (Basel), 8.

PROCHOWNIK, E. V. \& KUKOWSKA, J. 1986. Deregulated expression of c-myc by murine erythroleukaemia cells prevents differentiation. Nature, 322, 84850.

PUI, C. H., KANE, J. R. \& CRIST, W. M. 1995. Biology and treatment of infant leukemias. Leukemia, 9, 762-9.

PURI, T., WENDLER, P., SIGALA, B., SAIBIL, H. \& TSANEVA, I. R. 2007. Dodecameric structure and ATPase activity of the human TIP48/TIP49 complex. J Mol Biol, 366, 179-92.

QI, D., JIN, H., LILJA, T. \& MANNERVIK, M. 2006. Drosophila Reptin and other TIP60 complex components promote generation of silent chromatin. Genetics, 174, 241-51.

QIU, X. B., LIN, Y. L., THOME, K. C., PIAN, P., SCHLEGEL, B. P., WEREMOWICZ, S., PARVIN, J. D. \& DUTTA, A. 1998. An eukaryotic RuvB-like protein (RUVBL1) essential for growth. J Biol Chem, 273, 2778693.

QUELEN, C., LIPPERT, E., STRUSKI, S., DEMUR, C., SOLER, G., PRADE, N., DELABESSE, E., BROCCARDO, C., DASTUGUE, N., MAHON, F. X. \& BROUSSET, P. 2011. Identification of a transforming MYB-GATA1 fusion gene in acute basophilic leukemia: a new entity in male infants. Blood, 117, 5719-22. 
QUEVAL, R., PAPIN, C., DALVAI, M., BYSTRICKY, K. \& HUMBERT, O. 2014. Reptin and Pontin oligomerization and activity are modulated through histone H3 N-terminal tail interaction. J Biol Chem, 289, 33999-4012.

RADOVIC, S., RAPISARDA, V. A., TOSATO, V. \& BRUSCHI, C. V. 2007. Functional and comparative characterization of Saccharomyces cerevisiae RVB1 and RVB2 genes with bacterial Ruv homologues. FEMS Yeast Res, 7, 527-39.

RADTKE, I., MULLIGHAN, C. G., ISHII, M., SU, X., CHENG, J., MA, J., GANTI, R., CAI, Z., GOORHA, S., POUNDS, S. B., CAO, X., OBERT, C., ARMSTRONG, J., ZHANG, J., SONG, G., RIBEIRO, R. C., RUBNITZ, J. E., RAIMONDI, S. C., SHURTLEFF, S. A. \& DOWNING, J. R. 2009. Genomic analysis reveals few genetic alterations in pediatric acute myeloid leukemia. Proc Natl Acad Sci U S A, 106, 12944-9.

RAHL, P. B., LIN, C. Y., SEILA, A. C., FLYNN, R. A., MCCUINE, S., BURGE, C. B., SHARP, P. A. \& YOUNG, R. A. 2010. c-Myc regulates transcriptional pause release. Cell, 141, 432-45.

RAJENDRA, E., GARAYCOECHEA, J. I., PATEL, K. J. \& PASSMORE, L. A. 2014. Abundance of the Fanconi anaemia core complex is regulated by the RuvBL1 and RuvBL2 AAA+ ATPases. Nucleic Acids Res, 42, 13736-48.

RAMSAY, R. G. \& GONDA, T. J. 2008. MYB function in normal and cancer cells. Nat Rev Cancer, 8, 523-34.

RAMSAY, R. G., THOMPSON, M. A., HAYMAN, J. A., REID, G., GONDA, T. J. \& WHITEHEAD, R. H. 1992. Myb expression is higher in malignant human colonic carcinoma and premalignant adenomatous polyps than in normal mucosa. Cell Growth Differ, 3, 723-30.

RANDALL, T. D., LUND, F. E., HOWARD, M. C. \& WEISSMAN, I. L. 1996. Expression of murine CD38 defines a population of long-term reconstituting hematopoietic stem cells. Blood, 87, 4057-67.

RAVENS, S., YU, C., YE, T., STIERLE, M. \& TORA, L. 2015. Tip60 complex binds to active Pol II promoters and a subset of enhancers and co-regulates the c-Myc network in mouse embryonic stem cells. Epigenetics Chromatin, 8, 45.

REN, J., LI, W., LIU, H., YAN, L., JIAO, W., LI, D., TANG, Y., GU, G. \& XU, Z. 2013. Overexpression of reptin in renal cell carcinoma contributes to tumor malignancies and its inhibition triggers senescence of cancer cells. Urol Oncol, 31, 1358-66.

RODRIGUEZ-UBREVA, J. \& BALLESTAR, E. 2014. Chromatin immunoprecipitation. Methods Mol Biol, 1094, 309-18.

ROUSSEAU, B., MENARD, L., HAURIE, V., TARAS, D., BLANC, J. F., MOREAU-GAUDRY, F., METZLER, P., HUGUES, M., BOYAULT, S., LEMIERE, S., CANRON, X., COSTET, P., COLE, M., BALABAUD, C., BIOULAC-SAGE, P., ZUCMAN-ROSSI, J. \& ROSENBAUM, J. 2007. Overexpression and role of the ATPase and putative DNA helicase RuvB-like 2 in human hepatocellular carcinoma. Hepatology, 46, 1108-18.

RUBNITZ, J. E. \& INABA, H. 2012. Childhood acute myeloid leukaemia. $\mathrm{Br} J$ Haematol, 159, 259-76.

SALZER, U., KUBICEK, M. \& PROHASKA, R. 1999. Isolation, molecular characterization, and tissue-specific expression of ECP-51 and ECP-54 
(TIP49), two homologous, interacting erythroid cytosolic proteins. Biochim Biophys Acta, 1446, 365-70.

SANDBERG, M. L., SUTTON, S. E., PLETCHER, M. T., WILTSHIRE, T., TARANTINO, L. M., HOGENESCH, J. B. \& COOKE, M. P. 2005. c-Myb and p300 regulate hematopoietic stem cell proliferation and differentiation. Dev Cell, 8, 153-66.

SAYGIN, C. \& CARRAWAY, H. E. 2017. Emerging therapies for acute myeloid leukemia. J Hematol Oncol, 10, 93.

SCHMID, P., SCHULZ, W. A. \& HAMEISTER, H. 1989. Dynamic expression pattern of the myc protooncogene in midgestation mouse embryos. Science, 243, 226-9.

SCHMIDT, M., NAZAROV, V., STEVENS, L., WATSON, R. \& WOLFF, L. 2000. Regulation of the resident chromosomal copy of c-myc by c-Myb is involved in myeloid leukemogenesis. Mol Cell Biol, 20, 1970-81.

SCHNEIDER, A., PEUKERT, K., EILERS, M. \& HANEL, F. 1997. Association of Myc with the zinc-finger protein Miz-1 defines a novel pathway for gene regulation by Myc. Curr Top Microbiol Immunol, 224, 137-46.

SCHREINER, S., BIRKE, M., GARCIA-CUELLAR, M. P., ZILLES, O., GREIL, J. \& SLANY, R. K. 2001. MLL-ENL causes a reversible and myc-dependent block of myelomonocytic cell differentiation. Cancer Res, 61, 6480-6.

SCHUHMACHER, M., KOHLHUBER, F., HOLZEL, M., KAISER, C., BURTSCHER, H., JARSCH, M., BORNKAMM, G. W., LAUX, G., POLACK, A., WEIDLE, U. H. \& EICK, D. 2001. The transcriptional program of a human B cell line in response to Myc. Nucleic Acids Res, 29, 397-406.

SCHUHMACHER, M., STAEGE, M. S., PAJIC, A., POLACK, A., WEIDLE, U. H., BORNKAMM, G. W., EICK, D. \& KOHLHUBER, F. 1999. Control of cell growth by c-Myc in the absence of cell division. Curr Biol, 9, 1255-8.

SELVAKUMARAN, M., LIEBERMANN, D. A. \& HOFFMAN-LIEBERMANN, B. 1992. Deregulated c-myb disrupts interleukin-6- or leukemia inhibitory factor-induced myeloid differentiation prior to c-myc: role in leukemogenesis. Mol Cell Biol, 12, 2493-500.

SHEINESS, D. \& GARDINIER, M. 1984. Expression of a proto-oncogene (protomyb) in hemopoietic tissues of mice. Mol Cell Biol, 4, 1206-12.

SHEN, X., MIZUGUCHI, G., HAMICHE, A. \& WU, C. 2000. A chromatin remodelling complex involved in transcription and DNA processing. Nature, 406, 541-4.

SI, J., YU, X., ZHANG, Y. \& DEWILLE, J. W. 2010. Myc interacts with Max and Miz1 to repress C/EBPdelta promoter activity and gene expression. Mol Cancer, 9, 92.

SIRVEN, A., RAVET, E., CHARNEAU, P., ZENNOU, V., COULOMBEL, L., GUETARD, D., PFLUMIO, F. \& DUBART-KUPPERSCHMITT, A. 2001. Enhanced transgene expression in cord blood CD34(+)-derived hematopoietic cells, including developing $\mathrm{T}$ cells and NOD/SCID mouse repopulating cells, following transduction with modified trip lentiviral vectors. Mol Ther, 3, 43848.

SLANY, R. K. 2005. When epigenetics kills: MLL fusion proteins in leukemia. Hematol Oncol, 23, 1-9. 
SLANY, R. K. 2009. The molecular biology of mixed lineage leukemia. Haematologica, 94, 984-93.

SMITH, E., LIN, C. \& SHILATIFARD, A. 2011. The super elongation complex (SEC) and MLL in development and disease. Genes Dev, 25, 661-72.

SOMERVAILLE, T. C. \& CLEARY, M. L. 2006. Identification and characterization of leukemia stem cells in murine MLL-AF9 acute myeloid leukemia. Cancer Cell, 10, 257-68.

SOMERVAILlE, T. C., MATHENY, C. J., SPENCER, G. J., IWASAKI, M., RINN, J. L., WITTEN, D. M., CHANG, H. Y., SHURTLEFF, S. A., DOWNING, J. R. \& CLEARY, M. L. 2009. Hierarchical maintenance of MLL myeloid leukemia stem cells employs a transcriptional program shared with embryonic rather than adult stem cells. Cell Stem Cell, 4, 129-40.

SUBRAMANIAN, A., TAMAYO, P., MOOTHA, V. K., MUKHERJEE, S., EBERT, B. L., GILlETTE, M. A., PAUlOVICH, A., POMEROY, S. L., GOLUB, T. R., LANDER, E. S. \& MESIROV, J. P. 2005. Gene set enrichment analysis: a knowledge-based approach for interpreting genomewide expression profiles. Proc Natl Acad Sci U S A, 102, 15545-50.

SUMNER, R., CRAWFORD, A., MUCENSKI, M. \& FRAMPTON, J. 2000. Initiation of adult myelopoiesis can occur in the absence of c-Myb whereas subsequent development is strictly dependent on the transcription factor. Oncogene, 19, 3335-42.

SUZUKI, H., FORREST, A. R., VAN NIMWEGEN, E., DAUB, C. O., BALWIERZ, P. J., IRVINE, K. M., LASSMANN, T., RAVASI, T., HASEGAWA, Y., DE HOON, M. J., KATAYAMA, S., SCHRODER, K., CARNINCI, P., TOMARU, Y., KANAMORI-KATAYAMA, M., KUBOSAKI, A., AKALIN, A., ANDO, Y., ARNER, E., ASADA, M., ASAHARA, H., BAILEY, T., BAJIC, V. B., BAUER, D., BECKHOUSE, A. G., BERTIN, N., BJORKEGREN, J., BROMBACHER, F., BULGER, E., CHALK, A. M., CHIBA, J., CLOONAN, N., DAWE, A., DOSTIE, J., ENGSTROM, P. G., ESSACK, M., FAULKNER, G. J., FINK, J. L., FREDMAN, D., FUJIMORI, K., FURUNO, M., GOJOBORI, T., GOUGH, J., GRIMMOND, S. M., GUSTAFSSON, M., HASHIMOTO, M., HASHIMOTO, T., HATAKEYAMA, M., HEINZEL, S., HIDE, W., HOFMANN, O., HORNQUIST, M., HUMINIECKI, L., IKEO, K., IMAMOTO, N., INOUE, S., INOUE, Y., ISHIHARA, R., IWAYANAGI, T., JACOBSEN, A., KAUR, M., KAWAJI, H., KERR, M. C., KIMURA, R., KIMURA, S., KIMURA, Y., KITANO, H., KOGA, H., KOJIMA, T., KONDO, S., KONNO, T., KROGH, A., KRUGER, A., KUMAR, A., LENHARD, B., LENNARTSSON, A., LINDOW, M., LIZIO, M., MACPHERSON, C., MAEDA, N., MAHER, C. A., MAQUNGO, M., MAR, J., MATIGIAN, N. A., MATSUDA, H., MATTICK, J. S., MEIER, S., MIYAMOTO, S., MIYAMOTO-SATO, E., NAKABAYASHI, K., NAKACHI, Y., NAKANO, M., NYGAARD, S., OKAYAMA, T., OKAZAKI, Y., OKUDA-YABUKAMI, H., ORLANDO, V., OTOMO, J., PACHKOV, M., PETROVSKY, N., et al. 2009. The transcriptional network that controls growth arrest and differentiation in a human myeloid leukemia cell line. Nat Genet, 41, 553-62. 
TAKAHASHI, T., SUWABE, N., DAI, P., YAMAMOTO, M., ISHII, S. \& NAKANO, T. 2000. Inhibitory interaction of c-Myb and GATA-1 via transcriptional co-activator CBP. Oncogene, 19, 134-40.

TARANGELO, A., LO, N., TENG, R., KIM, E., LE, L., WATSON, D., FURTH, E. E., RAMAN, P., EHMER, U. \& VIATOUR, P. 2015. Recruitment of Pontin/Reptin by E2f1 amplifies E2f transcriptional response during cancer progression. Nat Commun, 6, 10028.

TAUSSIG, D. C., MIRAKI-MOUD, F., ANJOS-AFONSO, F., PEARCE, D. J., ALlEN, K., RIDLER, C., LILlINGTON, D., OAKERVEE, H., CAVENAGH, J., AGRAWAL, S. G., LISTER, T. A., GRIBBEN, J. G. \& BONNET, D. 2008. Anti-CD38 antibody-mediated clearance of human repopulating cells masks the heterogeneity of leukemia-initiating cells. Blood, $112,568-75$.

TERWIJN, M., ZEIJLEMAKER, W., KELDER, A., RUTTEN, A. P., SNEL, A. N., SCHOLTEN, W. J., PABST, T., VERHOEF, G., LOWENBERG, B., ZWEEGMAN, S., OSSENKOPPELE, G. J. \& SCHUURHUIS, G. J. 2014. Leukemic stem cell frequency: a strong biomarker for clinical outcome in acute myeloid leukemia. PLoS One, 9, e107587.

THOMPSON, C. B., CHALlONER, P. B., NEIMAN, P. E. \& GROUDINE, M. 1985. Levels of c-myc oncogene mRNA are invariant throughout the cell cycle. Nature, 314, 363-6.

UTTARKAR, S., FRAMPTON, J. \& KLEMPNAUER, K. H. 2017. Targeting the transcription factor Myb by small-molecule inhibitors. Exp Hematol, 47, 3135.

VARDIMAN, J. W., THIELE, J., ARBER, D. A., BRUNNING, R. D., BOROWITZ, M. J., PORWIT, A., HARRIS, N. L., LE BEAU, M. M., HELLSTROMLINDBERG, E., TEFFERI, A. \& BLOOMFIELD, C. D. 2009. The 2008 revision of the World Health Organization (WHO) classification of myeloid neoplasms and acute leukemia: rationale and important changes. Blood, 114, 937-51.

VENTEICHER, A. S., MENG, Z., MASON, P. J., VEENSTRA, T. D. \& ARTANDI, S. E. 2008. Identification of ATPases pontin and reptin as telomerase components essential for holoenzyme assembly. Cell, 132, 945-57.

VERVOORTS, J., LUSCHER-FIRZLAFF, J. \& LUSCHER, B. 2006. The ins and outs of MYC regulation by posttranslational mechanisms. J Biol Chem, 281, 34725-9.

VERVOORTS, J., LUSCHER-FIRZLAFF, J. M., ROTTMANN, S., LILISCHKIS, R., WALSEMANN, G., DOHMANN, K., AUSTEN, M. \& LUSCHER, B. 2003. Stimulation of c-MYC transcriptional activity and acetylation by recruitment of the cofactor CBP. EMBO Rep, 4, 484-90.

WALF-VORDERWÜLBECKE, V. 2009. A model to investigate the oncogenic activity of MLL-fusions in Acute Myeloid Leukaemia. University College London.

WALF-VORDERWULBECKE, V., DE BOER, J., HORTON, S. J., VAN AMERONGEN, R., PROOST, N., BERNS, A. \& WILLIAMS, O. 2012. Frat 2 mediates the oncogenic activation of Rac by MLL fusions. Blood, 120, 4819-28. 
WALKER, J. E., SARASTE, M., RUNSWICK, M. J. \& GAY, N. J. 1982. Distantly related sequences in the alpha- and beta-subunits of ATP synthase, myosin, kinases and other ATP-requiring enzymes and a common nucleotide binding fold. Embo j, 1, 945-51.

WALTER, R. B., OTHUS, M., BURNETT, A. K., LOWENBERG, B., KANTARJIAN, H. M., OSSENKOPPELE, G. J., HILLS, R. K., VAN MONTFORT, K. G., RAVANDI, F., EVANS, A., PIERCE, S. R., APPELBAUM, F. R. \& ESTEY, E. H. 2013. Significance of FAB subclassification of "acute myeloid leukemia, NOS" in the 2008 WHO classification: analysis of 5848 newly diagnosed patients. Blood, 121, 242431.

WALZ, S., LORENZIN, F., MORTON, J., WIESE, K. E., VON EYSS, B., HEROLD, S., RYCAK, L., DUMAY-ODELOT, H., KARIM, S., BARTKUHN, M., ROELS, F., WUSTEFELD, T., FISCHER, M., TEICHMANN, M., ZENDER, L., WEI, C. L., SANSOM, O., WOLF, E. \& EILERS, M. 2014. Activation and repression by oncogenic MYC shape tumour-specific gene expression profiles. Nature, 511, 483-7.

WANG, Z., GERSTEIN, M. \& SNYDER, M. 2009. RNA-Seq: a revolutionary tool for transcriptomics. Nat Rev Genet, 10, 57-63.

WEISKE, J. \& HUBER, O. 2005. The histidine triad protein Hint1 interacts with Pontin and Reptin and inhibits TCF-beta-catenin-mediated transcription. $J$ Cell Sci, 118, 3117-29.

WESTIN, E. H., GALLO, R. C., ARYA, S. K., EVA, A., SOUZA, L. M., BALUDA, M. A., AARONSON, S. A. \& WONG-STAAL, F. 1982. Differential expression of the amv gene in human hematopoietic cells. Proc Natl Acad Sci $U S$ A, 79, 2194-8.

WILSON, A., MURPHY, M. J., OSKARSSON, T., KALOULIS, K., BETTESS, M. D., OSER, G. M., PASCHE, A. C., KNABENHANS, C., MACDONALD, H. R. \& TRUMPP, A. 2004. c-Myc controls the balance between hematopoietic stem cell self-renewal and differentiation. Genes Dev, 18, 2747-63.

WINTERS, A. C. \& BERNT, K. M. 2017. MLL-Rearranged Leukemias-An Update on Science and Clinical Approaches. Front Pediatr, 5, 4.

WOLF, E., LIN, C. Y., EILERS, M. \& LEVENS, D. L. 2015. Taming of the beast: shaping Myc-dependent amplification. Trends Cell Biol, 25, 241-8.

WONG, P., IWASAKI, M., SOMERVAILLE, T. C., SO, C. W. \& CLEARY, M. L. 2007. Meis1 is an essential and rate-limiting regulator of MLL leukemia stem cell potential. Genes Dev, 21, 2762-74.

WOOD, M. A., MCMAHON, S. B. \& COLE, M. D. 2000. An ATPase/helicase complex is an essential cofactor for oncogenic transformation by c-Myc. Mol Cell, 5, 321-30.

XIANG, Z., LUO, H., PAYTON, J. E., CAIN, J., LEY, T. J., OPFERMAN, J. T. \& TOMASSON, M. H. 2010. Mcl1 haploinsufficiency protects mice from Mycinduced acute myeloid leukemia. J Clin Invest, 120, 2109-18.

YAMAMOTO, R., MORITA, Y., OOEHARA, J., HAMANAKA, S., ONODERA, M., RUDOLPH, K. L., EMA, H. \& NAKAUCHI, H. 2013. Clonal analysis unveils self-renewing lineage-restricted progenitors generated directly from hematopoietic stem cells. Cell, 154, 1112-26. 
YANAGISAWA, H., NAGASAWA, T., KURAMOCHI, S., ABE, T., IKAWA, Y. \& TODOKORO, K. 1991. Constitutive expression of exogenous c-myb gene causes maturation block in monocyte-macrophage differentiation. Biochim Biophys Acta, 1088, 380-4.

YEOH, E. J., ROSS, M. E., SHURTLEFF, S. A., WILLIAMS, W. K., PATEL, D., MAHFOUZ, R., BEHM, F. G., RAIMONDI, S. C., RELLING, M. V., PATEL, A., CHENG, C., CAMPANA, D., WILKINS, D., ZHOU, X., LI, J., LIU, H., PUI, C. H., EVANS, W. E., NAEVE, C., WONG, L. \& DOWNING, J. R. 2002. Classification, subtype discovery, and prediction of outcome in pediatric acute lymphoblastic leukemia by gene expression profiling. Cancer Cell, 1, 133-43.

YOKOYAMA, A. \& CLEARY, M. L. 2008. Menin critically links MLL proteins with LEDGF on cancer-associated target genes. Cancer Cell, 14, 36-46.

ZEISIG, B. B., KULASEKARARAJ, A. G., MUFTI, G. J. \& SO, C. W. 2012. SnapShot: Acute myeloid leukemia. Cancer Cell, 22, 698-698.e1.

ZEISIG, B. B., MILNE, T., GARCIA-CUELLAR, M. P., SCHREINER, S., MARTIN, M. E., FUCHS, U., BORKHARDT, A., CHANDA, S. K., WALKER, J., SODEN, R., HESS, J. L. \& SLANY, R. K. 2004. Hoxa9 and Meis 1 are key targets for MLL-ENL-mediated cellular immortalization. Mol Cell Biol, 24, 617-28.

ZELEZNIK-LE, N. J., HARDEN, A. M. \& ROWLEY, J. D. 1994. 11q23 translocations split the "AT-hook" cruciform DNA-binding region and the transcriptional repression domain from the activation domain of the mixedlineage leukemia (MLL) gene. Proc Natl Acad Sci U S A, 91, 10610-4.

ZELLER, K. I., ZHAO, X., LEE, C. W., CHIU, K. P., YAO, F., YUSTEIN, J. T., OOI, H. S., ORLOV, Y. L., SHAHAB, A., YONG, H. C., FU, Y., WENG, Z., KUZNETSOV, V. A., SUNG, W. K., RUAN, Y., DANG, C. V. \& WEI, C. L. 2006. Global mapping of c-Myc binding sites and target gene networks in human B cells. Proc Natl Acad Sci U S A, 103, 17834-9.

ZHAO, H., JIN, S. \& GEWIRTZ, A. M. 2012. The histone acetyltransferase TIP60 interacts with c-Myb and inactivates its transcriptional activity in human leukemia. J Biol Chem, 287, 925-34.

ZHAO, L. 2013. Identifying critical signalling pathways in MLL-rearranged acute myeloid leukaemia. PhD Thesis, University College London.

ZHAO, L., GLAZOV, E. A., PATTABIRAMAN, D. R., AL-OWAIDI, F., ZHANG, P., BROWN, M. A., LEO, P. J. \& GONDA, T. J. 2011. Integrated genomewide chromatin occupancy and expression analyses identify key myeloid prodifferentiation transcription factors repressed by Myb. Nucleic Acids Res, 39, 4664-79.

ZHAO, L., YE, P. \& GONDA, T. J. 2014. The MYB proto-oncogene suppresses monocytic differentiation of acute myeloid leukemia cells via transcriptional activation of its target gene GFI1. Oncogene, 33, 4442-9.

ZHOU, C. Y., STODDARD, C. I., JOHNSTON, J. B., TRNKA, M. J., ECHEVERRIA, I., PALOVCAK, E., SALI, A., BURLINGAME, A. L., CHENG, Y. \& NARLIKAR, G. J. 2017. Regulation of Rvb1/Rvb2 by a Domain within the INO80 Chromatin Remodeling Complex Implicates the Yeast Rvbs as Protein Assembly Chaperones. Cell Rep, 19, 2033-2044. 
ZHOU, Y. \& NESS, S. A. 2011. Myb proteins: angels and demons in normal and transformed cells. Front Biosci (Landmark Ed), 16, 1109-31.

ZUBER, J., RAPPAPORT, A. R., LUO, W., WANG, E., CHEN, C., VASEVA, A. V., SHI, J., WEISSMUELLER, S., FELLMANN, C., TAYLOR, M. J., WEISSENBOECK, M., GRAEBER, T. G., KOGAN, S. C., VAKOC, C. R. \& LOWE, S. W. 2011. An integrated approach to dissecting oncogene addiction implicates a Myb-coordinated self-renewal program as essential for leukemia maintenance. Genes Dev, 25, 1628-40. 


\section{APPENDIX}

Table 14. List of the 100 most upregulated and downregulated genes after 4 days of inducible Reptin depletion.

\begin{tabular}{|c|c|c|c|}
\hline \multicolumn{2}{|c|}{$\begin{array}{c}\text { UPREGULATED } \\
\text { GENES }\end{array}$} & \multicolumn{2}{|c|}{$\begin{array}{c}\text { DOWNREGULATED } \\
\text { GENES }\end{array}$} \\
\hline GENE & FOLD & GENE & FOLD \\
\hline SYMBOL & CHANGE & SYMBOL & CHANGE \\
\hline MIR30A & 15.032 & RUVBL2 & -3.364 \\
\hline LOX & 9.514 & PCOLCE2 & -2.949 \\
\hline SIGLEC1 & 6.774 & VIT & -2.751 \\
\hline PRR16 & 6.727 & TUB & -2.676 \\
\hline ZNF608 & 5.098 & TERT & -2.657 \\
\hline ANTXR1 & 4.347 & LRP4 & -2.549 \\
\hline MX1 & 4.347 & C1orf95 & -2.497 \\
\hline IFI44L & 4.317 & DLGAP1-AS3 & -2.462 \\
\hline DUSP4 & 4.056 & UGT3A2 & -2.462 \\
\hline IFI6 & 4.028 & FAM135B & -2.378 \\
\hline OAS3 & 3.630 & SFXN2 & -2.362 \\
\hline ABLIM3 & 3.605 & $\mathrm{CHDH}$ & -2.329 \\
\hline CORO2B & 3.364 & DKFZ & -2.329 \\
\hline TNXB & 3.340 & LIPG & -2.282 \\
\hline
\end{tabular}




\begin{tabular}{|c|c|c|c|}
\hline SERPINI1 & 3.272 & MS4A3 & -2.282 \\
\hline VWA5A & 3.227 & C20orf197 & -2.266 \\
\hline CLCN5 & 3.227 & WNT7B & -2.266 \\
\hline LGALS3BP & 3.204 & PRTN3 & -2.266 \\
\hline SH3TC2 & 3.160 & CAV1 & -2.235 \\
\hline RPE65 & 3.138 & FAM203A & -2.219 \\
\hline TENM3 & 3.095 & MARC1 & -2.173 \\
\hline FUCA1 & 3.053 & PRRT4 & -2.173 \\
\hline DUSP27 & 3.053 & TCN1 & -2.158 \\
\hline OAS1 & 3.053 & WWC1 & -2.158 \\
\hline EPSTI1 & 3.053 & NUDT8 & -2.085 \\
\hline OAS2 & 3.010 & BMP8B & -2.071 \\
\hline IFI27 & 3.010 & LRIG3 & -2.071 \\
\hline IFITM1 & 2.928 & COL24A1 & -2.042 \\
\hline KHDRBS2 & 2.928 & B4GALT2 & -2.028 \\
\hline IFI44 & 2.868 & JAM3 & -2.028 \\
\hline STAP1 & 2.828 & MPZL1 & -2.014 \\
\hline IFIT1 & 2.809 & MIR210HG & -2.014 \\
\hline $\mathrm{KCNJ} 2$ & 2.809 & RRM2 & -1.988 \\
\hline TXLNB & 2.809 & TP63 & -1.975 \\
\hline GBP2 & 2.751 & SULF2 & -1.974 \\
\hline IER5L & 2.751 & CPNE7 & -1.970 \\
\hline ADAM9 & 2.732 & PKP2 & -1.967 \\
\hline ITGAM & 2.639 & NAV3 & -1.963 \\
\hline
\end{tabular}




\begin{tabular}{|c|c|c|c|}
\hline NRP2 & 2.639 & PRLR & -1.959 \\
\hline TG & 2.621 & PLAC8 & -1.956 \\
\hline TNFRSF11A & 2.603 & FLT3 & -1.952 \\
\hline MAML3 & 2.603 & ENHO & -1.947 \\
\hline DLL4 & 2.585 & GAS6 & -1.941 \\
\hline ADCY4 & 2.567 & KIAA1644 & -1.941 \\
\hline ATP8B1 & 2.549 & USH1C & -1.937 \\
\hline TUBB3 & 2.514 & DHCR7 & -1.937 \\
\hline SLC22A18AS & 2.497 & RAB37 & -1.937 \\
\hline LEPR & 2.462 & HAL & -1.928 \\
\hline IFIT3 & 2.462 & FAM69B & -1.920 \\
\hline CD82 & 2.462 & C16orf74 & -1.909 \\
\hline OASL & 2.445 & DHRS11 & -1.907 \\
\hline CD52 & 2.428 & PTPRS & -1.907 \\
\hline CNKSR3 & 2.428 & SERPINE2 & -1.901 \\
\hline IFITM3 & 2.412 & FADS1 & -1.895 \\
\hline BZRAP1 & 2.412 & TMEM45A & -1.895 \\
\hline ABCA9 & 2.378 & LPL & -1.893 \\
\hline IRF9 & 2.362 & GLO1 & -1.891 \\
\hline ISG15 & 2.313 & DSC3 & -1.880 \\
\hline ZMAT3 & 2.313 & PACSIN3 & -1.878 \\
\hline STX11 & 2.313 & CGREF1 & -1.878 \\
\hline SEPT4 & 2.297 & PAQR5 & -1.871 \\
\hline DNAJB2 & 2.297 & AP1S3 & -1.870 \\
\hline
\end{tabular}




\begin{tabular}{|c|c|c|c|}
\hline MMP2 & 2.282 & LOC254559 & -1.862 \\
\hline S1PR5 & 2.266 & FGFR4 & -1.852 \\
\hline LILRB2 & 2.266 & CNN3 & -1.847 \\
\hline LRRC3B & 2.266 & TCEAL2 & -1.844 \\
\hline SESN1 & 2.266 & SLC29A2 & -1.840 \\
\hline RDH10 & 2.250 & LOC730091 & -1.838 \\
\hline KIAA1324 & 2.204 & KATNAL2 & -1.835 \\
\hline TUFT1 & 2.204 & GLDC & -1.826 \\
\hline DTNA & 2.204 & SPRYD4 & -1.816 \\
\hline IL17RE & 2.204 & PEX5L & -1.811 \\
\hline GPNMB & 2.204 & FERMT1 & -1.810 \\
\hline LOC100294362 & 2.189 & METTL21A & -1.808 \\
\hline REC8 & 2.173 & CTSL2 & -1.803 \\
\hline F2RL3 & 2.173 & FBXO43 & -1.800 \\
\hline VSIG4 & 2.173 & KLRG2 & -1.799 \\
\hline MTMR9LP & 2.158 & SLC38A5 & -1.795 \\
\hline FERMT2 & 2.158 & IMPDH2 & -1.794 \\
\hline YPEL3 & 2.158 & ZFP2 & -1.785 \\
\hline SAMSN1 & 2.158 & CCBL1 & -1.785 \\
\hline SGPP1 & 2.144 & CAV2 & -1.783 \\
\hline EMR4P & 2.129 & SLC25A10 & -1.780 \\
\hline IFIT2 & 2.114 & SSPN & -1.774 \\
\hline IFI30 & 2.114 & AK4 & -1.772 \\
\hline SIGLEC11 & 2.099 & MTG1 & -1.770 \\
\hline
\end{tabular}




\begin{tabular}{cc|cc}
\hline OPTN & 2.085 & SIRPB2 & -1.770 \\
ABHD4 & 2.085 & PFN2 & -1.770 \\
ALDH8A1 & 2.085 & DLEU2 & -1.767 \\
SEMA6B & 2.085 & LOC100506472 & -1.764 \\
STXBP5-AS1 & 2.071 & HIST1H2BH & -1.761 \\
TTC21A & 2.056 & C6orf223 & -1.750 \\
SYTL3 & 2.056 & SMO & -1.748 \\
TCHH & 2.042 & POU4F2 & -1.746 \\
ABCB11 & 2.042 & RCC1 & -1.744 \\
\hline IRF7 & 2.028 & ELOVL2 & -1.744 \\
\hline SLFN5 & 2.028 & ACAT2 & -1.744 \\
\hline MEGF10 & 2.028 & SLC7A2 & -1.742 \\
\hline B3GALT4 & 2.028 & CSPG5 & -1.741 \\
\hline FIG4 & 2.028 & F2R & -1.740 \\
\hline
\end{tabular}


Table 15. List of the 100 most upregulated and downregulated genes after 7 days of constitutive Reptin depletion.

\begin{tabular}{|c|c|c|c|}
\hline \multicolumn{2}{|c|}{$\begin{array}{c}\text { UPREGULATED } \\
\text { GENES }\end{array}$} & \multicolumn{2}{|c|}{$\begin{array}{l}\text { DOWNREGULATED } \\
\text { GENES }\end{array}$} \\
\hline GENE & FOLD & GENE & FOLD \\
\hline SYMBOL & CHANGE & SYMBOL & CHANGE \\
\hline RP11-96K19.2 & 5.389 & RUVBL2 & -45.255 \\
\hline AF127936.7 & 5.278 & C20orf197 & -4.112 \\
\hline CRIP2 & 3.138 & STK36 & -3.811 \\
\hline LEPR & 2.868 & MFAP4 & -3.732 \\
\hline HIST1H2BK & 2.868 & $\begin{array}{l}\text { RP11- } \\
352 \mathrm{D} 3.2\end{array}$ & -3.630 \\
\hline ANKDD1A & 2.770 & MAMDC4 & -3.531 \\
\hline RP11-326K13.4 & 2.770 & KCTD15 & -3.482 \\
\hline AC007204.2 & 2.621 & CENPE & -3.387 \\
\hline ACTG2 & 2.514 & TEX15 & -3.294 \\
\hline KCNJ1 & 2.514 & SYTL1 & -3.249 \\
\hline MEF2BNB & 2.514 & C1RL & -3.204 \\
\hline DNAJB2 & 2.462 & TESPA1 & -3.074 \\
\hline PRR4 & 2.462 & NFKBIZ & -2.969 \\
\hline SOX7 & 2.395 & PTK7 & -2.969 \\
\hline SDCBP2 & 2.362 & MKI67 & -2.949 \\
\hline
\end{tabular}




\begin{tabular}{|c|c|c|c|}
\hline TBX19 & 2.346 & INHBA-AS 1 & -2.908 \\
\hline HIST3H2A & 2.313 & ANKRD18A & -2.888 \\
\hline AC104653.1 & 2.313 & ORM1 & -2.828 \\
\hline RP11-438E5.1 & 2.313 & TRAF1 & -2.809 \\
\hline CD82 & 2.297 & PSAT1 & -2.789 \\
\hline HMOX1 & 2.297 & $\begin{array}{c}\text { RP11- } \\
701 \mathrm{H} 24.2\end{array}$ & -2.789 \\
\hline MAP1LC3A & 2.235 & CCDC78 & -2.789 \\
\hline RHBDD2 & 2.219 & WBP2NL & -2.789 \\
\hline ADORA3 & 2.189 & HAAO & -2.732 \\
\hline F11R & 2.173 & BEND6 & -2.732 \\
\hline AC006967.1 & 2.173 & TSKU & -2.732 \\
\hline GPR89B & 2.158 & CPNE7 & -2.732 \\
\hline FHIT & 2.158 & FBLN1 & -2.732 \\
\hline GJC2 & 2.144 & CCDC18 & -2.713 \\
\hline ZMAT3 & 2.085 & AC083843.1 & -2.713 \\
\hline AKR1C3 & 2.071 & KCNS1 & -2.713 \\
\hline $\begin{array}{l}\text { FAM47E- } \\
\text { STBD1 }\end{array}$ & 2.056 & $\begin{array}{l}\text { HNRNPU- } \\
\text { AS1 }\end{array}$ & -2.694 \\
\hline RAB3C & 2.056 & NGEF & -2.694 \\
\hline HIST2H2BE & 2.042 & SAP25 & -2.694 \\
\hline LRRC3B & 2.042 & CTSK & -2.676 \\
\hline CHCHD6 & 2.028 & AC011625.1 & -2.676 \\
\hline AC106722.1 & 2.028 & RP11-53B2.2 & -2.621 \\
\hline
\end{tabular}




\begin{tabular}{|c|c|c|c|}
\hline RHOC & 2.014 & COL27A1 & -2.621 \\
\hline OR2W3 & 2.000 & PAPLN & -2.603 \\
\hline CAMKMT & 2.000 & SMC1B & -2.603 \\
\hline ACOT2 & 2.000 & MALAT1 & -2.603 \\
\hline NPTX1 & 1.988 & SLC7A11 & -2.585 \\
\hline RP11-184I16.4 & 1.977 & ENPP2 & -2.585 \\
\hline OVGP1 & 1.970 & $\begin{array}{c}\text { RP11- } \\
\text { 110I1.12 }\end{array}$ & -2.585 \\
\hline BMPER & 1.970 & RP11-368I7.2 & -2.585 \\
\hline TBC1D7 & 1.964 & NEAT1 & -2.585 \\
\hline PHLDA2 & 1.957 & NRBP2 & -2.567 \\
\hline IFI30 & 1.953 & TRIM66 & -2.567 \\
\hline SERTAD1 & 1.952 & $\begin{array}{c}\text { RP11- } \\
\text { 392A22.2 }\end{array}$ & -2.549 \\
\hline MB21D2 & 1.945 & TMEM80 & -2.549 \\
\hline CRISPLD2 & 1.941 & NICN1 & -2.532 \\
\hline LYPD5 & 1.935 & PLEKHH1 & -2.532 \\
\hline ALDH8A1 & 1.931 & SCN4A & -2.532 \\
\hline HIST1H2BD & 1.903 & $\begin{array}{c}\text { RP4- } \\
\text { 694B14.5 }\end{array}$ & -2.532 \\
\hline SLCO2B1 & 1.903 & $\begin{array}{c}\text { MCM3AP- } \\
\text { AS1 }\end{array}$ & -2.532 \\
\hline NME2 & 1.903 & RFX8 & -2.514 \\
\hline EMP1 & 1.897 & AGBL3 & -2.514 \\
\hline
\end{tabular}




\begin{tabular}{|c|c|c|c|}
\hline $\begin{array}{c}\text { RP11- } \\
\text { 1094M14.11 }\end{array}$ & 1.892 & CBS & -2.514 \\
\hline PPP1R32 & 1.880 & ADAMTS13 & -2.497 \\
\hline TSPAN15 & 1.875 & SEPT5 & -2.497 \\
\hline TLR5 & 1.871 & IL2RB & -2.497 \\
\hline NEU1 & 1.871 & $\begin{array}{c}\text { RP5- } \\
1180 \mathrm{C} 10.2\end{array}$ & -2.479 \\
\hline OCLN & 1.870 & ANK2 & -2.479 \\
\hline HIST1H2AC & 1.867 & CENPF & -2.462 \\
\hline 37316 & 1.865 & DCAF16 & -2.462 \\
\hline S100A10 & 1.863 & MORC4 & -2.445 \\
\hline ZNF607 & 1.862 & $\begin{array}{c}\text { RP11- } \\
\text { 262H14.1 }\end{array}$ & -2.428 \\
\hline AC109826.1 & 1.861 & MS4A4E & -2.428 \\
\hline HRH1 & 1.860 & CEP290 & -2.428 \\
\hline CD5L & 1.858 & DNHD1 & -2.428 \\
\hline PGM2L1 & 1.857 & AC084018.1 & -2.428 \\
\hline RP11-48O20.4 & 1.851 & LINC00638 & -2.412 \\
\hline PLS1 & 1.844 & GPT2 & -2.412 \\
\hline CTSL1 & 1.844 & SLC6A9 & -2.412 \\
\hline SNX11 & 1.840 & PAQR6 & -2.412 \\
\hline TMEM38A & 1.837 & ZMAT1 & -2.412 \\
\hline VAMP5 & 1.835 & EBF1 & -2.395 \\
\hline $\mathrm{CA} 2$ & 1.834 & RP11-95P2.1 & -2.395 \\
\hline
\end{tabular}




\begin{tabular}{|c|c|c|c|}
\hline CA13 & 1.829 & SIGLEC6 & -2.395 \\
\hline MIP & 1.829 & C9orf139 & -2.395 \\
\hline TRMT11 & 1.828 & PRAM1 & -2.395 \\
\hline MSR1 & 1.828 & PLA2G6 & -2.378 \\
\hline P4HA2 & 1.826 & AL449209.1 & -2.362 \\
\hline SELM & 1.825 & VEGFA & -2.362 \\
\hline RP11-568N6.1 & 1.823 & AKAP9 & -2.362 \\
\hline RP11-214O1.2 & 1.823 & ASNS & -2.362 \\
\hline SLC25A5-AS1 & 1.823 & RAD52 & -2.362 \\
\hline AL645728.1 & 1.821 & ARHGAP33 & -2.362 \\
\hline SEPW1 & 1.821 & ZNF587B & -2.362 \\
\hline C4orf48 & 1.820 & SEMG1 & -2.362 \\
\hline C7orf55 & 1.820 & NPIP & -2.362 \\
\hline TP53INP1 & 1.819 & USP46-AS1 & -2.346 \\
\hline LGMN & 1.815 & GRB10 & -2.346 \\
\hline AC079767.4 & 1.811 & ERV3-1 & -2.346 \\
\hline HLA-F-AS1 & 1.809 & ATHL1 & -2.346 \\
\hline ATP6V1D & 1.809 & OXER1 & -2.329 \\
\hline KLHL25 & 1.808 & PEX5L & -2.329 \\
\hline FPR2 & 1.808 & RHPN1 & -2.329 \\
\hline ALDH3A2 & 1.798 & SFXN2 & -2.329 \\
\hline HUS1 & 1.795 & ANKRD24 & -2.329 \\
\hline
\end{tabular}

